Supplementary Information for

\title{
Solvent Effect on the Sergeants-and-Soldiers Effect Leading to Bidirectional Induction of Single-Handed Helical Sense of Poly(quinoxaline-2,3-diyl)s Copolymers in Aromatic Solvents
}

Yuuya Nagata, Tsuyoshi Nishikawa, and Michinori Suginome*

Department of Synthetic Chemistry and Biological Chemistry, Graduate School of Engineering, Kyoto University, Kyoto 606-8501, Japan

615-8510, Japan

*To whom correspondence should be addressed.

E-mail: suginome@sbchem.kyoto-u.ac.jp

\section{Contents}

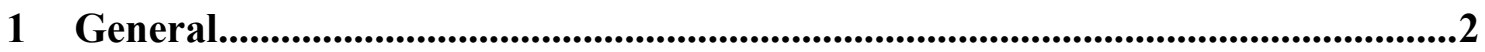

2 Experimental Procedures and Spectral Data for New Compounds .....................3

3 Use of Polymer 4 as a Chiral Ligand in the Asymmetric Suzuki-Miyaura

Cross-Coupling Reaction ...................................................................................11

4 Chiroptical Properties of Polymer Solutions ....................................................16

5 UV-vis Absorption and CD Spectra of New Compounds .................................19

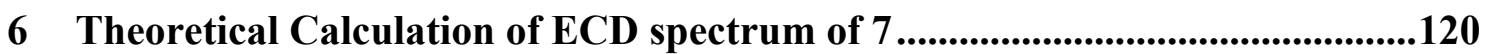

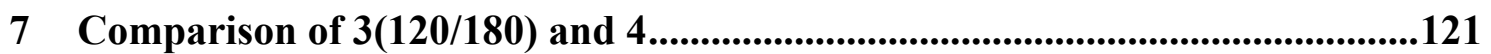

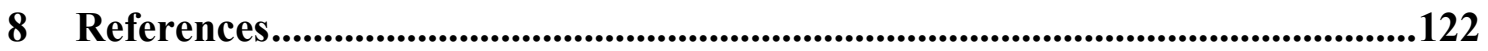

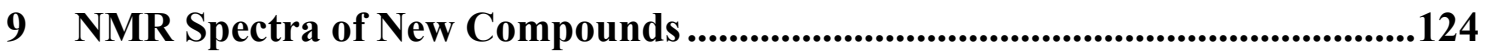




\section{General}

All reactions were carried out under an atmosphere of nitrogen with magnetic stirring. ${ }^{1} \mathrm{H}$ NMR spectra were recorded on a Varian 400-MR (400 MHz) spectrometer at ambient temperature. ${ }^{13} \mathrm{C}$ NMR spectra were recorded on a Varian 400-MR (100 MHz) spectrometer at ambient temperature. ${ }^{31} \mathrm{P}$ NMR spectra were recorded on a Varian 400-MR (125 MHz) spectrometer at ambient temperature. ${ }^{1} \mathrm{H}$ NMR data are reported as follows: chemical shift in ppm downfield from tetramethylsilane ( $\delta$ scale), multiplicity ( $\mathrm{s}=$ singlet, $\mathrm{d}=$ doublet, $\mathrm{t}=$ triplet, $\mathrm{q}=$ quartet, quint $=$ quintet, $\mathrm{sex}=$ sextet, $\mathrm{m}=$ multiplet and $\mathrm{br}=$ broad $)$, coupling constant $(\mathrm{Hz})$, and integration. ${ }^{13} \mathrm{C}$ NMR chemical shifts are reported in ppm downfield from tetramethylsilane ( $\delta$ scale). ${ }^{31} \mathrm{P}$ NMR chemical shifts are reported in ppm downfield from $\mathrm{H}_{3} \mathrm{PO}_{4}(85 \%)$. The GPC analysis was carried out with TSKgel $\mathrm{GMH}_{\mathrm{XL}}\left(\mathrm{CHCl}_{3}\right.$, polystyrene standards). Preparative GPC was performed on JAI LC-908 equipped with JAIGEL-1H and -2H columns in a series $\left(\mathrm{CHCl}_{3}\right)$. UV-vis absorption spectra were recorded on a JASCO V-750 spectrometer equipped with a JASCO ETC-505T temperature/stirring controller at $20{ }^{\circ} \mathrm{C}$. Circular dichroism (CD) spectra were recorded on a JASCO J-1500 spectrometer equipped with a JASCO PTC-510 temperature/stirring controller at $20^{\circ} \mathrm{C}$. The chiral HPLC analysis was carried out on TOSOH 8020 series equipped with CHIRALCEL ${ }^{\circledR}$ OZ-H (hexane and 2-propanol). Flash chromatography was performed using a Biotage Isolera One flash purification system with silica gel flash cartridges.

Tetrahydrofurane (THF) was dried and deoxygenized using an alumina/catalyst column system (Glass Contour Co.). Benzene $(\mathrm{PhH})$ and benzotrifluoride $\left(\mathrm{PhCF}_{3}\right)$ were distilled over $\mathrm{CaH}_{2}$ and degassed prior to use. Trichlorosilane was distilled over quinoline prior to use.

$o$-TolNiCl( $\left.\mathrm{PMe}_{3}\right)_{2},{ }^{1} \quad$ 1,2-bis((S)-sec-butoxymethyl)-4,5-diisocyano-3,6-dimethylben-zene (Qa) ${ }^{2}$ 1,2-diisocyano-3,6-dimethyl-4,5-bis((S)-2-methylbutoxy)benzene (Qb), ${ }^{3}$ 1,2-diisocyano-3,6-dimethyl-4,5-bis((((S)-octan-2-yl)oxy)methyl)benzene (Qc) ${ }^{4}$ 1,2-di-isocyano-3,6-dimethyl-4,5-bis((((S)-octan-3-yl)oxy)methyl)benzene $\quad(\mathbf{Q d}){ }^{4} \quad$ 1,2-di-butoxy-4,5-diisocyano-3,6-dimethylbenzene (Q2), ${ }^{5}$ 1,2-diisocyano-3,6-dimethyl-4,5bis(propoxymethyl)benzene (Q3), ${ }^{6}$ and (2',3'-diisocyano-4'-methyl-[1,1'-biphenyl]-2yl)diphenylphosphine sulfide (Qp) ${ }^{7} \mathbf{1 a ( 1 0 0 )},{ }^{8} \mathbf{1 b}(\mathbf{1 0 0}),{ }^{8} \mathbf{1 c}(\mathbf{1 0 0}),{ }^{8} \mathbf{1 d}(\mathbf{1 0 0}),{ }^{8} \mathbf{2 a ( 5 / 1 0 0 )},{ }^{9}$ $\mathbf{2 d}(\mathbf{5} / \mathbf{1 0 0}),{ }^{9} \mathbf{3 a ( 5 / 1 0 0 )},{ }^{9} \mathbf{3 d}(/ \mathbf{1 0 0}),{ }^{9} \mathbf{3 d}(\mathbf{2 . 5 / 9 7 . 5}),{ }^{9} \mathbf{3 d}(\mathbf{5} / \mathbf{9 5}),{ }^{9} \mathbf{3 d}(\mathbf{7 . 5 / 9 2 . 5}),{ }^{9} \mathbf{3 d}(\mathbf{1 0 / 9 0}),{ }^{9}$ 
$\mathbf{3 d}(\mathbf{1 5} / \mathbf{8 5}),{ }^{9} \quad \mathbf{3 d}(\mathbf{2 0} / \mathbf{8 0}),{ }^{9} \quad \mathbf{3 d}(\mathbf{4 0} / \mathbf{6 0}),{ }^{9} \quad \mathbf{3 d}(\mathbf{6 0} / \mathbf{4 0}),{ }^{9} \quad$ and $\quad \mathbf{3 d}(\mathbf{8 0} / \mathbf{2 0})^{9} \quad$ and dimethyl(1-bromonaphthalen-2-yl)phophonate $(\mathbf{5})^{10}$ were prepared according to the reported procedures. 4-Methyl-1-naphthaleneboronic acid (6) and other chemical reagents were purchased from the commercial sources and were used without further purification.

\section{Experimental Procedures and Spectral Data for New Compounds}

Synthesis of Polymer 2b(5/100): Qb (1.64 mg, $5.0 \mu \mathrm{mol})$ and Q2 (30.04 mg, 100.0 $\mu \mathrm{mol})$ were dissolved in THF (2 mL). A THF solution of $o$-TolNiCl( $\left.\mathrm{PMe}_{3}\right)_{2}(6.43 \mathrm{mM}$, $156 \mu \mathrm{L}, 1.0 \mu \mathrm{mol})$ was added to the monomer solution with vigorous stirring. After $16 \mathrm{~h}$, $\mathrm{NaBH}_{4}(7.94 \mathrm{mg}, 210 \mu \mathrm{mol})$ was added to the reaction mixture and stirred for $1 \mathrm{~h}$. Water $(10 \mathrm{~mL})$ was added to the solution and extracted with $\mathrm{CH}_{2} \mathrm{Cl}_{2}(15 \mathrm{ml})$. The organic extract was washed with brine $(10 \mathrm{~mL})$, dried over $\mathrm{Na}_{2} \mathrm{SO}_{4}$, and the solvent was evaporated. The residue was subjected to preparative GPC to give $\mathbf{2 b}$ as a beige solid (29.4 mg, 93\%). ${ }^{1} \mathrm{H}$ NMR $\left(\mathrm{CDCl}_{3}\right) \delta 4.00\left(\mathrm{H}_{\mathrm{b}-\mathrm{B}}\right.$ and $\mathrm{H}_{2-\mathrm{B}},(5 \times 2+100 \times 2) \mathrm{H}$, br s), 3.84 $\left(\mathrm{H}_{\mathrm{b}-\mathrm{B}}\right.$ and $\mathrm{H}_{2-\mathrm{B}},(5 \times 2+100 \times 2) \mathrm{H}$, br s), $2.17\left(\mathrm{H}_{\mathrm{b}-\mathrm{A}}, \mathrm{H}_{2-\mathrm{A}}\right.$ and $\mathrm{H}_{\mathrm{t}-\mathrm{B}},(5 \times 6+100 \times 6+3) \mathrm{H}$, br s$)$, $1.74\left(\mathrm{H}_{\mathrm{b}-\mathrm{C}}\right.$ and $\mathrm{H}_{2-\mathrm{C}}, \quad(5 \times 2+100 \times 4) \mathrm{H}, \quad$ br $\left.\mathrm{s}\right), \quad 1.60-1.32 \quad\left(\mathrm{H}_{\mathrm{b}-\mathrm{D}}\right.$ and $\mathrm{H}_{2-\mathrm{D}}$, $(5 \times 2+5 \times 2+100 \times 4) \mathrm{H}$, br m), $1.26\left(\mathrm{H}_{\mathrm{b}-\mathrm{D}}, 5 \times 2 \mathrm{H}\right.$, br s$), 0.96\left(\mathrm{H}_{\mathrm{b}-\mathrm{E}}, \mathrm{H}_{\mathrm{b}-\mathrm{F}}\right.$ and $\mathrm{H}_{2-\mathrm{E}}$, $(5 \times 6+5 \times 6+100 \times 6) \mathrm{H}, \mathrm{t}, J=7.0 \mathrm{~Hz})$, small peaks originated from end-group were observed in $10.27\left(\mathrm{H}_{\mathrm{t}-\mathrm{A}}, 1 \mathrm{H}, \mathrm{s}\right)$ and 7.82-7.10 ppm ( $\left.\mathrm{H}_{\mathrm{t}-\mathrm{C}}, 4 \mathrm{H}, \mathrm{m}\right)$; $\mathrm{GPC}\left(\mathrm{CHCl}_{3}, \mathrm{~g} / \mathrm{mol}\right)$ : $M_{\mathrm{n}}=2.80 \times 10^{4}, M_{\mathrm{w}} / M_{\mathrm{n}}=1.08$.

Scheme S1. Synthesis of polymer $3 b(5 / 100)$.

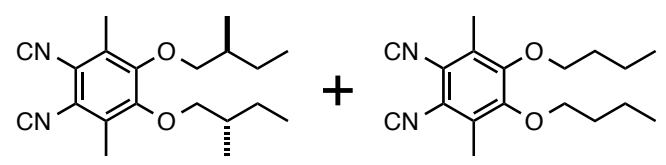

Qb (5 eq)

Q2 (100 eq)

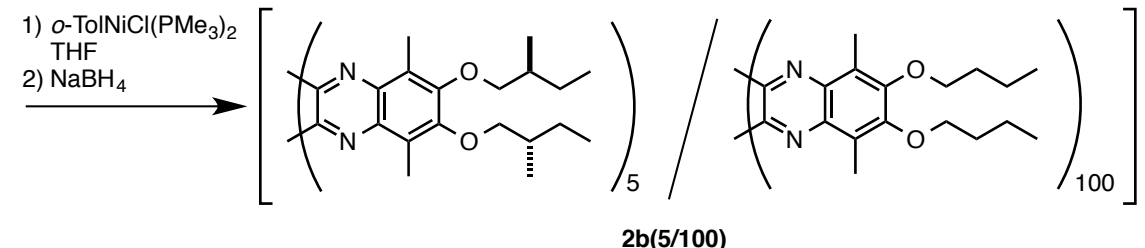



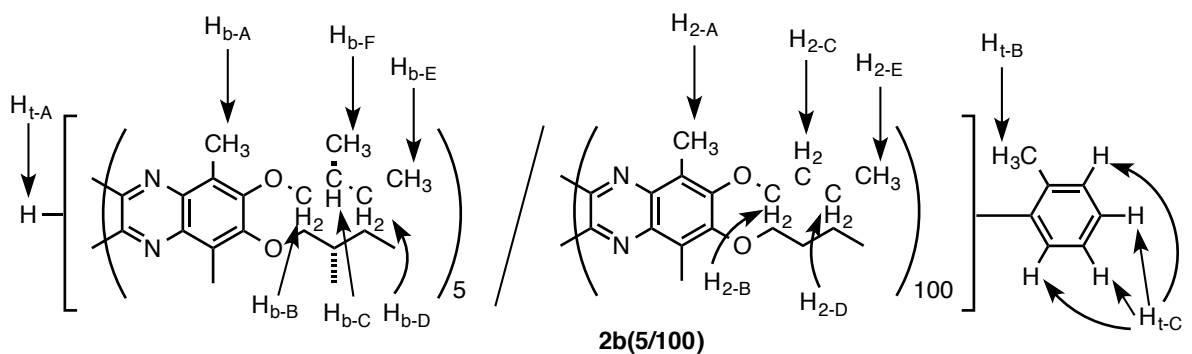

Figure S1. Structure of polymer $\mathbf{2 b ( 5 / 1 0 0 )}$ with ${ }^{1} \mathrm{H}$ NMR assignment.

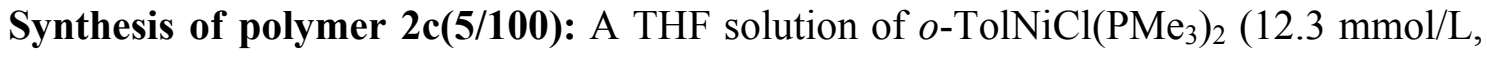
$131 \mu \mathrm{L}, 1.61 \mu \mathrm{mol})$ was added to $3.0 \mathrm{~mL}$ THF solution of Qc $(3.56 \mathrm{mg}, 8.08 \mu \mathrm{mol})$ and Q2 (48.55 mg, $161.6 \mu \mathrm{mol})$ with vigorous stirring. After $27 \mathrm{~h}, \mathrm{NaBH}_{4}(12.84 \mathrm{mg}, 339.3$ $\mu \mathrm{mol})$ was added to the reaction mixture and stirred for $1 \mathrm{~h}$. Water $(20 \mathrm{~mL})$ was added to the solution and extracted with $\mathrm{CH}_{2} \mathrm{Cl}_{2}(20 \mathrm{ml})$. The organic extract was washed with brine $(20 \mathrm{~mL})$, dried over $\mathrm{Na}_{2} \mathrm{SO}_{4}$, and the solvent was evaporated. The residue was subjected to preparative GPC to give $\mathbf{2 c ( 5 / 1 0 0 )}$ as a beige solid $(49.3 \mathrm{mg}, 95 \%)$. ${ }^{1} \mathrm{H}$ $\operatorname{NMR}\left(\mathrm{CDCl}_{3}\right) \delta 4.62\left(\mathrm{H}_{\mathrm{c}-\mathrm{B}}, 5 \times 2 \mathrm{H}\right.$, br s $), 4.51\left(\mathrm{H}_{\mathrm{c}-\mathrm{B}}, 5 \times 2 \mathrm{H}\right.$, br s $), 4.00\left(\mathrm{H}_{2-\mathrm{B}}, 100 \times 2 \mathrm{H}, \mathrm{br}\right.$ s), $3.84\left(\mathrm{H}_{2-\mathrm{B}}, 100 \times 2 \mathrm{H}\right.$, br s$), 3.50\left(\mathrm{H}_{\mathrm{c}-\mathrm{C}}, 5 \times 2 \mathrm{H}\right.$, br s$), 2.18\left(\mathrm{H}_{\mathrm{c}-\mathrm{A}}, \mathrm{H}_{2-\mathrm{A}}\right.$ and $\mathrm{H}_{\mathrm{t}-\mathrm{B}}$, $(5 \times 6+100 \times 6+3) \mathrm{H}$, br s $), 1.75\left(\mathrm{H}_{\mathrm{c}-\mathrm{D}}\right.$ and $\mathrm{H}_{2-\mathrm{C}}(5 \times 2+100 \times 4) \mathrm{H}$, br s $), 1.56-1.35\left(\mathrm{H}_{\mathrm{c}-\mathrm{E}}, \mathrm{H}_{\mathrm{c}-\mathrm{F}}\right.$, $\mathrm{H}_{\mathrm{c}-\mathrm{G}}$ and $\mathrm{H}_{2-\mathrm{D}},(5 \times 16+100 \times 4) \mathrm{H}$, br m), $1.23\left(\mathrm{H}_{\mathrm{c}-\mathrm{J}}, 5 \times 6 \mathrm{H}\right), 0.96\left(\mathrm{H}_{2-\mathrm{E}}, 100 \times 6 \mathrm{H}, \mathrm{t}, J=6.4\right.$ $\mathrm{Hz}), 0.79\left(\mathrm{H}_{\mathrm{c}-\mathrm{I}}, 5 \times 6 \mathrm{H}, \mathrm{br} \mathrm{s}\right)$, small peaks originated from end-group were observed in $9.98\left(\mathrm{H}_{\mathrm{t}-\mathrm{A}}, 1 \mathrm{H}, \mathrm{s}\right)$ and $7.84-7.12 \mathrm{ppm}\left(\mathrm{H}_{\mathrm{t}-\mathrm{C}}, 4 \mathrm{H}, \mathrm{m}\right) ; \mathrm{GPC}\left(\mathrm{CHCl}_{3}, \mathrm{~g} / \mathrm{mol}\right): M_{\mathrm{n}}=2.82 \times$ $10^{4}, M_{\mathrm{w}} / M_{\mathrm{n}}=1.11$.

Scheme S2. Synthesis of polymer $2 \mathrm{c}(\mathbf{5} / \mathbf{1 0 0})$.

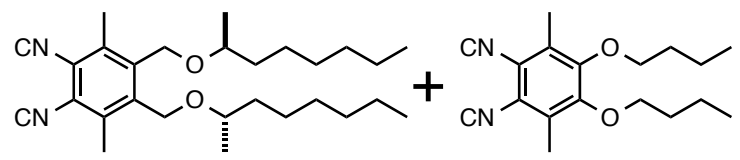

Qc (5 eq)

Q2 (100 eq)

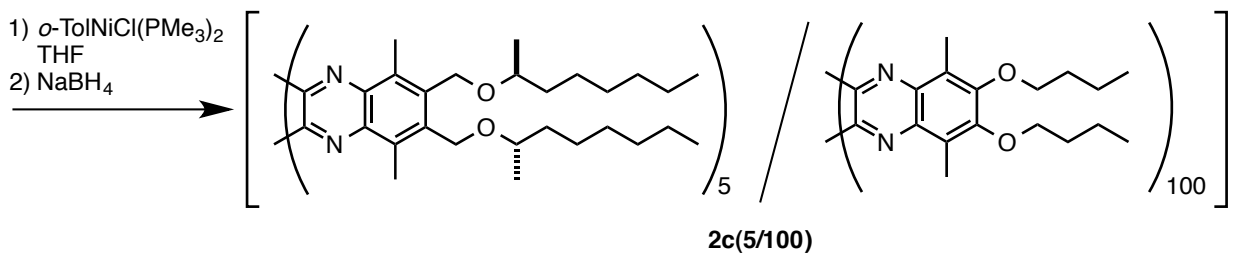

4 


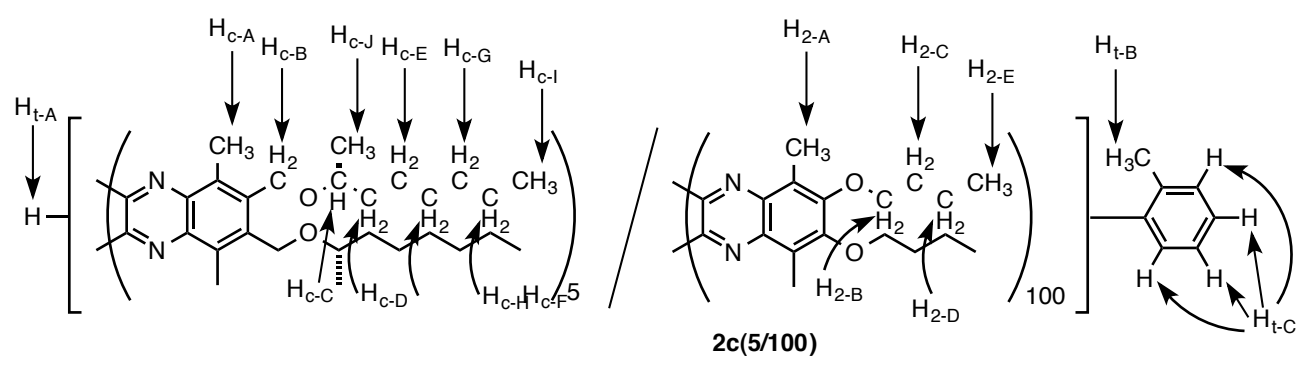

Figure S2. Structure of polymer 2c(5/100) with ${ }^{1} \mathrm{H}$ NMR assignment.

Synthesis of Polymer 3b(5/100): Qb $(1.64 \mathrm{mg}, 5.0 \mu \mathrm{mol})$ and Q3 $(30.04 \mathrm{mg}, 100.0$ $\mu \mathrm{mol})$ were dissolved in THF $(2 \mathrm{~mL})$. A THF solution of $o$-TolNiCl( $\left.\mathrm{PMe}_{3}\right)_{2}(6.43 \mathrm{mM}$, $156 \mu \mathrm{L}, 1.0 \mu \mathrm{mol}$ ) was added to the monomer solution with vigorous stirring. After $17 \mathrm{~h}$, $\mathrm{NaBH}_{4}(7.94 \mathrm{mg}, 210 \mu \mathrm{mol})$ was added to the reaction mixture and stirred for $1 \mathrm{~h}$. Water $(10 \mathrm{~mL})$ was added to the solution and extracted with $\mathrm{CH}_{2} \mathrm{Cl}_{2}(15 \mathrm{ml})$. The organic extract was washed with brine $(10 \mathrm{~mL})$, dried over $\mathrm{Na}_{2} \mathrm{SO}_{4}$, and the solvent was evaporated. The residue was subjected to preparative GPC to give $\mathbf{3 b}(\mathbf{5} / \mathbf{1 0 0})$ as a beige solid (31.5 mg, 99\%). ${ }^{1} \mathrm{H}$ NMR $\left(\mathrm{CDCl}_{3}\right) \delta 4.65\left(\mathrm{H}_{3-\mathrm{B}}, 100 \times 2 \mathrm{H}\right.$, br s$), 4.56\left(\mathrm{H}_{3-\mathrm{B}}\right.$, $100 \times 2 \mathrm{H}$, br s), $3.46\left(\mathrm{H}_{\mathrm{b}-\mathrm{B}}\right.$ and $\mathrm{H}_{3-\mathrm{C}},(5 \times 4+100 \times 4) \mathrm{H}$, br s), $2.35\left(\mathrm{H}_{\mathrm{b}-\mathrm{A}}, \mathrm{H}_{3-\mathrm{A}}\right.$ and $\mathrm{H}_{\mathrm{t}-\mathrm{B}}$, $(5 \times 6+100 \times 6+3) \mathrm{H}$, br s), $1.61-1.36\left(\mathrm{H}_{\mathrm{b}-\mathrm{C}}, \mathrm{H}_{\mathrm{b}-\mathrm{D}}\right.$ and $\mathrm{H}_{3-\mathrm{D}},(5 \times 2+5 \times 2+100 \times 4) \mathrm{H}$, br m), $1.26\left(\mathrm{H}_{\mathrm{b}-\mathrm{D}}, 5 \times 4 \mathrm{H}\right.$, br s), $1.05\left(\mathrm{H}_{\mathrm{b}-\mathrm{F}}, 5 \times 6 \mathrm{H}, \mathrm{d}, J=6.0 \mathrm{~Hz}\right), 0.90\left(\mathrm{H}_{\mathrm{b}-\mathrm{E}}\right.$ and $\mathrm{H}_{3-\mathrm{E}}$, $(5 \times 6+100 \times 6) \mathrm{H}, \mathrm{t}, J=6.4 \mathrm{~Hz})$, small peaks originated from end-group were observed in $10.30\left(\mathrm{H}_{\mathrm{t}-\mathrm{A}}, 1 \mathrm{H}, \mathrm{s}\right)$ and $7.86-7.09 \mathrm{ppm}\left(\mathrm{H}_{\mathrm{t}-\mathrm{C}}, 4 \mathrm{H}, \mathrm{m}\right) ; \mathrm{GPC}\left(\mathrm{CHCl}_{3}, \mathrm{~g} / \mathrm{mol}\right): M_{\mathrm{n}}=2.72 \times$ $10^{4}, M_{\mathrm{w}} / M_{\mathrm{n}}=1.09$.

Scheme S3. Synthesis of polymer $3 b(5 / 100)$.

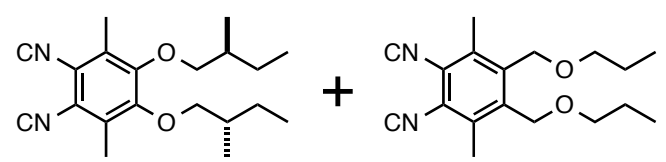

Qb (5 eq)

Q3 (100 eq)

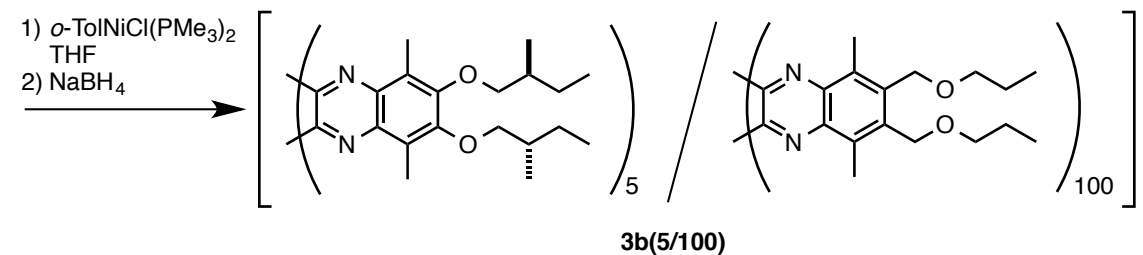




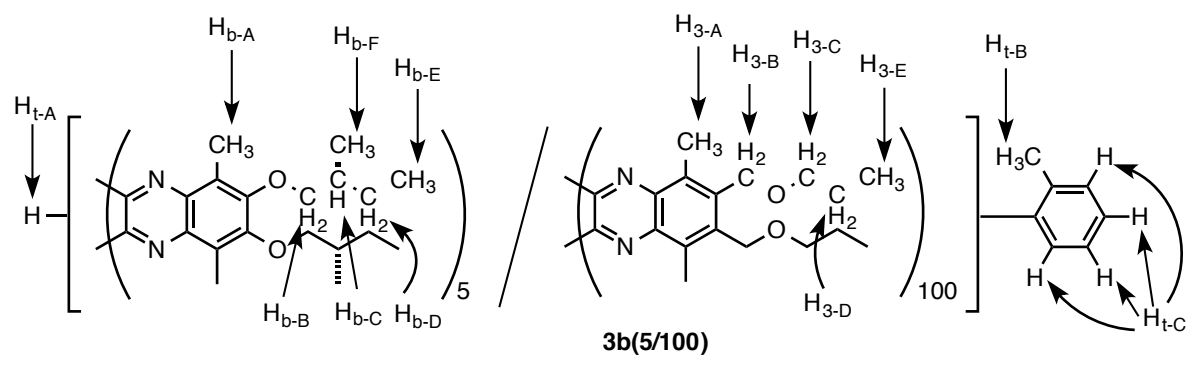

Figure S3. Structure of polymer $\mathbf{3 b}(\mathbf{5 / 1 0 0})$ with ${ }^{1} \mathrm{H}$ NMR assignment.

Synthesis of polymer 3c(5/100): A THF solution of $o$-TolNiCl( $\left.\mathrm{PMe}_{3}\right)_{2}(12.3 \mathrm{mmol} / \mathrm{L}$, $129 \mu \mathrm{L}, 1.58 \mu \mathrm{mol})$ was added to $3.0 \mathrm{~mL}$ THF solution of Qc $(3.49 \mathrm{mg}, 7.92 \mu \mathrm{mol})$ and Q3 (47.57 mg, $158.4 \mu \mathrm{mol})$ with vigorous stirring. After $27 \mathrm{~h}, \mathrm{NaBH}_{4}(12.58 \mathrm{mg}, 332.5$ $\mu \mathrm{mol})$ was added to the reaction mixture and stirred for $1 \mathrm{~h}$. Water $(20 \mathrm{~mL})$ was added to the solution and extracted with $\mathrm{CH}_{2} \mathrm{Cl}_{2}(20 \mathrm{ml})$. The organic extract was washed with brine $(20 \mathrm{~mL})$, dried over $\mathrm{Na}_{2} \mathrm{SO}_{4}$, and the solvent was evaporated. The residue was subjected to preparative GPC to give $\mathbf{3 c}(\mathbf{5} / \mathbf{1 0 0})$ as a beige solid $(36.5 \mathrm{mg}, 71 \%) .{ }^{1} \mathrm{H}$ $\operatorname{NMR}\left(\mathrm{CDCl}_{3}\right) \delta 4.57\left(\mathrm{H}_{\mathrm{c}-\mathrm{B}}\right.$ and $\mathrm{H}_{1-\mathrm{B}},(5 \times 4+100 \times 4) \mathrm{H}$, br s), $3.47\left(\mathrm{H}_{\mathrm{c}-\mathrm{C}}\right.$ and $\mathrm{H}_{1-\mathrm{C}}$, $(5 \times 2+100 \times 4) \mathrm{H}$, br s $), 2.35\left(\mathrm{H}_{\mathrm{c}-\mathrm{A}}, \mathrm{H}_{1-\mathrm{A}}\right.$ and $\mathrm{H}_{\mathrm{t}-\mathrm{B}},(5 \times 6+100 \times 6+3) \mathrm{H}$, br s $), 1.60\left(\mathrm{H}_{\mathrm{c}-\mathrm{D}}\right.$, and $\mathrm{H}_{1-\mathrm{D}},(5 \times 4+100 \times 4) \mathrm{H}$, br s), $1.26\left(\mathrm{H}_{\mathrm{c}-\mathrm{E}}, \mathrm{H}_{\mathrm{c}-\mathrm{F}}, \mathrm{H}_{\mathrm{c}-\mathrm{G}}\right.$ and $\mathrm{H}_{\mathrm{c}-\mathrm{H}}, 5 \times 16 \mathrm{H}$, br s$), 0.90\left(\mathrm{H}_{\mathrm{c}-\mathrm{J}}, \mathrm{H}_{\mathrm{c}-\mathrm{I}}\right.$ and $\mathrm{H}_{1-\mathrm{E}},(5 \times 12+100 \times 6) \mathrm{H}$, br s), small peaks originated from end-group were observed in $10.08\left(\mathrm{H}_{\mathrm{t}-\mathrm{A}}, 1 \mathrm{H}, \mathrm{s}\right)$ and $7.88-7.10 \mathrm{ppm}\left(\mathrm{H}_{\mathrm{t}-\mathrm{C}}, 4 \mathrm{H}, \mathrm{m}\right)$; $\mathrm{GPC}\left(\mathrm{CHCl}_{3}, \mathrm{~g} / \mathrm{mol}\right): M_{\mathrm{n}}=2.62$ $\times 10^{4}, M_{\mathrm{w}} / M_{\mathrm{n}}=1.11$.

Scheme S4. Synthesis of polymer 3(100).<smiles>CCCCCCC(C)OCc1c(C)c(N)c(C)c(COC(C)CCCCC)c1COCCC</smiles>

Qc (5 eq)

Q3 (100 eq)

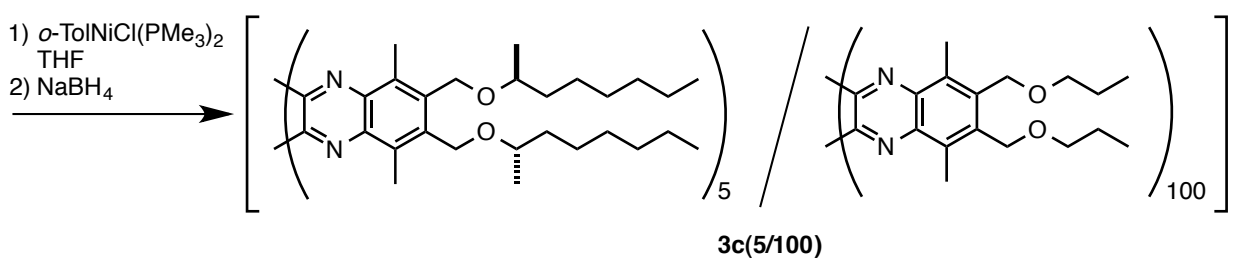

6 


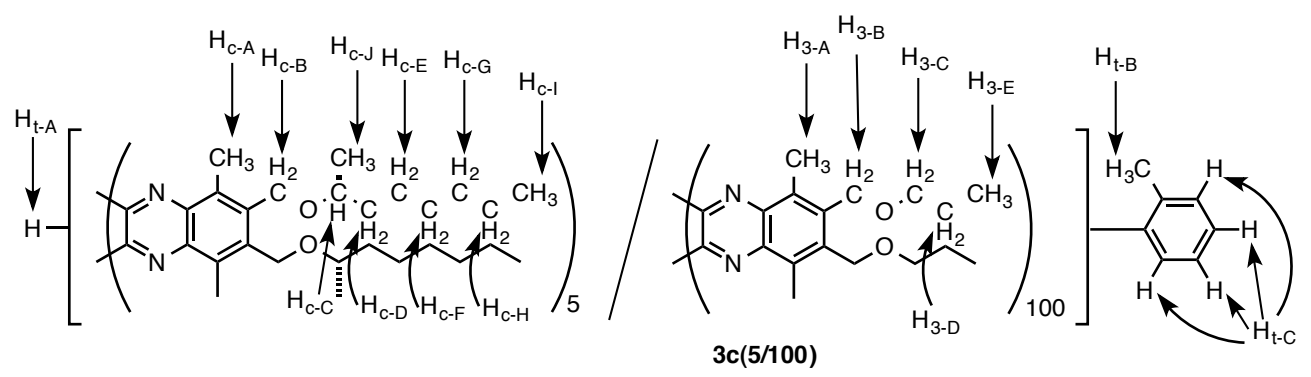

Figure S4. Structure of polymer $\mathbf{3 c ( 1 0 0 )}$ with ${ }^{1} \mathrm{H}$ NMR assignment.

Synthesis of polymer 3d(30/70): A THF solution of $o$-TolNiCl( $\left.\mathrm{PMe}_{3}\right)_{2}(8.96 \mathrm{mmol} / \mathrm{L}$, $89.3 \mu \mathrm{L}, 0.800 \mu \mathrm{mol})$ was added to $3.0 \mathrm{~mL}$ THF solution of Qd (10.58 mg, $24.0 \mu \mathrm{mol})$ and Q3 (16.82 mg, $56.0 \mu \mathrm{mol})$ with vigorous stirring. After $15 \mathrm{~h}, \mathrm{NaBH}_{4}(6.36 \mathrm{mg}, 168$ $\mu \mathrm{mol})$ was added to the reaction mixture and stirred for $1 \mathrm{~h}$. Water $(15 \mathrm{~mL})$ was added to the solution and extracted with $\mathrm{CH}_{2} \mathrm{Cl}_{2}(15 \mathrm{ml})$. The organic extract was washed with brine $(15 \mathrm{~mL})$, dried over $\mathrm{Na}_{2} \mathrm{SO}_{4}$, and the solvent was evaporated. The residue was subjected to preparative GPC to give 3d(30/70) as a beige solid $(24.5 \mathrm{mg}, 95 \%) .{ }^{1} \mathrm{H}$ $\operatorname{NMR}\left(\mathrm{CDCl}_{3}\right) \delta 4.58\left(\mathrm{H}_{\mathrm{d}-\mathrm{B}}\right.$ and $\mathrm{H}_{1-\mathrm{B}},(30 \times 4+70 \times 4) \mathrm{H}$, br s $), 3.45\left(\mathrm{H}_{1-\mathrm{C}}, 70 \times 4 \mathrm{H}\right.$, br s), $3.31\left(\mathrm{H}_{\mathrm{d}-\mathrm{C}}, 30 \times 2 \mathrm{H}\right.$, br s $), 2.34\left(\mathrm{H}_{\mathrm{d}-\mathrm{A}}, \mathrm{H}_{1-\mathrm{A}}\right.$ and $\mathrm{H}_{\mathrm{t}-\mathrm{B}},(30 \times 6+70 \times 6+3) \mathrm{H}$, br s$), 1.84-1.10$ $\left(\mathrm{H}_{\mathrm{d}-\mathrm{D}}, \mathrm{H}_{\mathrm{d}-\mathrm{E}}, \mathrm{H}_{\mathrm{d}-\mathrm{F}}, \mathrm{H}_{\mathrm{d}-\mathrm{G}}, \mathrm{H}_{\mathrm{d}-\mathrm{I}}\right.$ and $\mathrm{H}_{1-\mathrm{D}},(30 \times 20+70 \times 4) \mathrm{H}$, br m), $0.89\left(\mathrm{H}_{\mathrm{d}-\mathrm{H}}, \mathrm{H}_{\mathrm{d}-\mathrm{J}}\right.$ and $\mathrm{H}_{1-\mathrm{E}}$, $(30 \times 12+70 \times 6) \mathrm{H}$, br s), small peaks originated from end-group were observed in 10.03 $\left(\mathrm{H}_{\mathrm{t}-\mathrm{A}}, 1 \mathrm{H}, \mathrm{s}\right)$ and 7.87-7.10ppm $\left(\mathrm{H}_{\mathrm{t}-\mathrm{C}}, 4 \mathrm{H}, \mathrm{m}\right) ; \mathrm{GPC}\left(\mathrm{CHCl}_{3}, \mathrm{~g} / \mathrm{mol}\right): M_{\mathrm{n}}=1.65 \times 10^{4}$, $M_{\mathrm{w}} / M_{\mathrm{n}}=1.01$.

Scheme S5. Synthesis of polymer 3d(x/y).
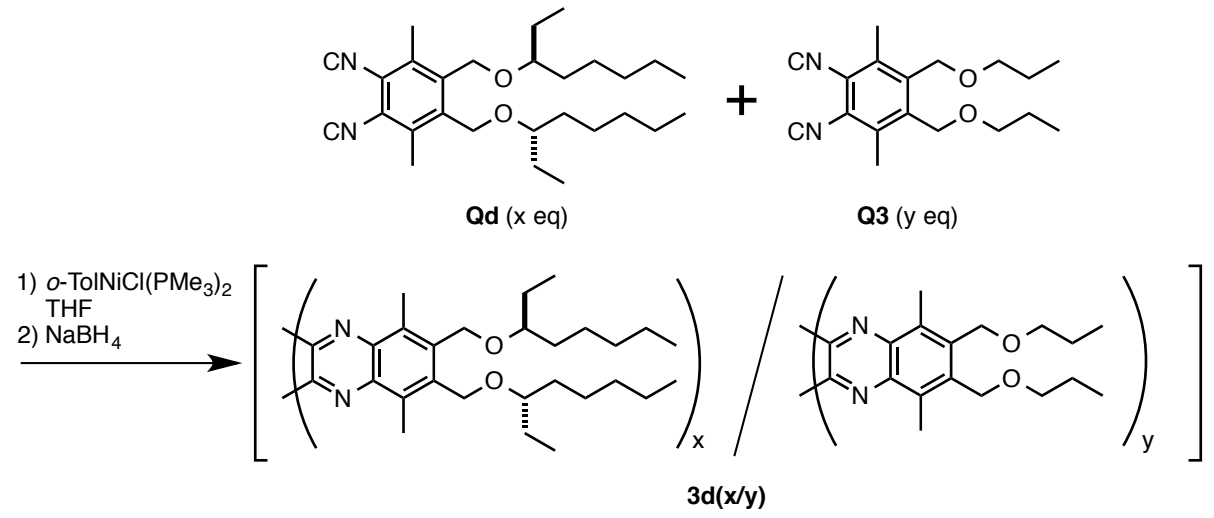


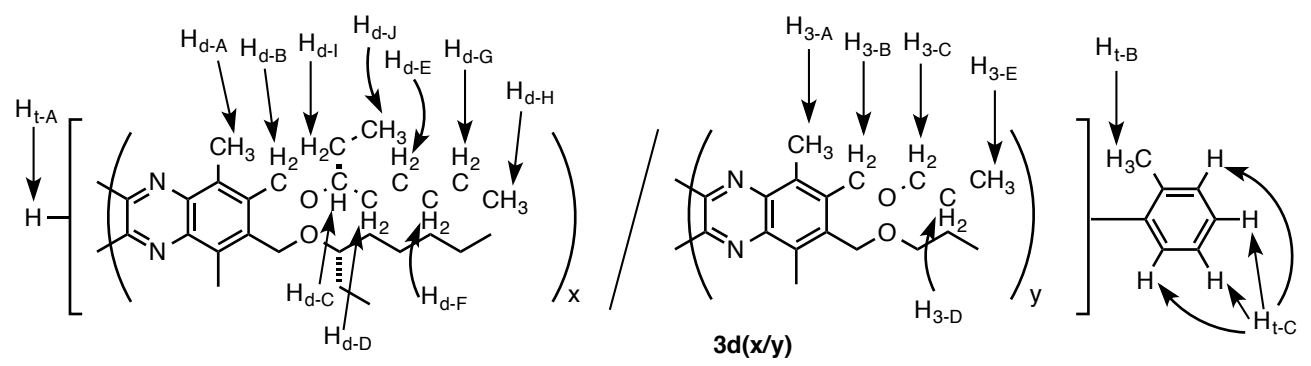

Figure S5. Structure of polymer $\mathbf{3 d}(\mathbf{x} / \mathbf{y})$ with ${ }^{1} \mathrm{H}$ NMR assignment.

Synthesis of Polymer 3d(20/30): A THF solution of $o$-TolNiCl( $\left.\mathrm{PMe}_{3}\right)_{2}(12.34 \mathrm{mmol} / \mathrm{L}$, $101 \mu \mathrm{L}, 1.25 \mu \mathrm{mol})$ was added to $3.0 \mathrm{~mL}$ THF solution of Qd (11.02 mg, $25.0 \mu \mathrm{mol})$ and Q3 $(11.27 \mathrm{mg}, 37.5 \mu \mathrm{mol})$ with vigorous stirring. After $27 \mathrm{~h}, \mathrm{NaBH}_{4}(9.93 \mathrm{mg}$, $262.5 \mu \mathrm{mol})$ was added to the reaction mixture and stirred for $1 \mathrm{~h}$. Water $(15 \mathrm{~mL})$ was added to the solution and extracted with $\mathrm{CH}_{2} \mathrm{Cl}_{2}(15 \mathrm{ml})$. The organic extract was washed with brine $(15 \mathrm{~mL})$, dried over $\mathrm{Na}_{2} \mathrm{SO}_{4}$, and the solvent was evaporated. The residue was subjected to preparative GPC to give $\mathbf{3 d}(\mathbf{2 0} / \mathbf{3 0})$ as a beige solid $(19.7 \mathrm{mg}$, $88 \%) .{ }^{1} \mathrm{H}$ NMR $\left(\mathrm{CDCl}_{3}\right) \delta 4.57\left(\mathrm{H}_{\mathrm{d}-\mathrm{B}}\right.$ and $\mathrm{H}_{1-\mathrm{B}},(20 \times 4+30 \times 4) \mathrm{H}$, br s$), 3.45\left(\mathrm{H}_{1-\mathrm{C}}\right.$, $30 \times 4 \mathrm{H}$, br s $), 3.31\left(\mathrm{H}_{\mathrm{d}-\mathrm{C}}, 20 \times 2 \mathrm{H}\right.$, br s $), 2.33\left(\mathrm{H}_{\mathrm{d}-\mathrm{A}}, \mathrm{H}_{1-\mathrm{A}}\right.$ and $\mathrm{H}_{\mathrm{t}-\mathrm{B}},(20 \times 6+30 \times 6+3) \mathrm{H}, \mathrm{br}$ s), $1.70-1.18\left(\mathrm{H}_{\mathrm{d}-\mathrm{D}}, \mathrm{H}_{\mathrm{d}-\mathrm{E}}, \mathrm{H}_{\mathrm{d}-\mathrm{F}}, \mathrm{H}_{\mathrm{d}-\mathrm{G}}, \mathrm{H}_{\mathrm{d}-\mathrm{I}}\right.$ and $\mathrm{H}_{1-\mathrm{D}},(20 \times 20+30 \times 4) \mathrm{H}$, br m), $0.88\left(\mathrm{H}_{\mathrm{d}-\mathrm{H}}\right.$, $\mathrm{H}_{\text {d-J }}$ and $\mathrm{H}_{1-\mathrm{E}},(20 \times 12+30 \times 6) \mathrm{H}$, br s), small peaks originated from end-group were observed in $10.17\left(\mathrm{H}_{\mathrm{t}-\mathrm{A}}, 1 \mathrm{H}, \mathrm{s}\right)$ and 7.86-7.09ppm $\left(\mathrm{H}_{\mathrm{t}-\mathrm{C}}, 4 \mathrm{H}, \mathrm{m}\right)$; $\mathrm{GPC}\left(\mathrm{CHCl}_{3}, \mathrm{~g} / \mathrm{mol}\right)$ : $M_{\mathrm{n}}=1.43 \times 10^{4}, M_{\mathrm{w}} / M_{\mathrm{n}}=1.11$.

Synthesis of polymer 3d(60/90): A THF solution of $o$-TolNiCl( $\left.\mathrm{PMe}_{3}\right)_{2}(12.34 \mathrm{mmol} / \mathrm{L}$, $33.8 \mu \mathrm{L}, 0.417 \mu \mathrm{mol})$ was added to $3.0 \mathrm{~mL}$ THF solution of Qd (11.02 $\mathrm{mg}, 25.0 \mu \mathrm{mol})$ and Q3 $(11.27 \mathrm{mg}, 37.5 \mu \mathrm{mol})$ with vigorous stirring. After $27 \mathrm{~h}, \mathrm{NaBH}_{4}(3.31 \mathrm{mg}, 87.5$ $\mu \mathrm{mol})$ was added to the reaction mixture and stirred for $1 \mathrm{~h}$. Water $(15 \mathrm{~mL})$ was added to the solution and extracted with $\mathrm{CH}_{2} \mathrm{Cl}_{2}(15 \mathrm{ml})$. The organic extract was washed with brine $(15 \mathrm{~mL})$, dried over $\mathrm{Na}_{2} \mathrm{SO}_{4}$, and the solvent was evaporated. The residue was subjected to preparative GPC to give $\mathbf{3 d}(\mathbf{6 0 / 9 0})$ as a beige solid $(18.5 \mathrm{mg}, 83 \%) .{ }^{1} \mathrm{H}$ NMR $\left(\mathrm{CDCl}_{3}\right) \delta 4.67\left(\mathrm{H}_{\mathrm{d}-\mathrm{B}}\right.$ and $\mathrm{H}_{1-\mathrm{B}},(60 \times 4+90 \times 4) \mathrm{H}$, br s $), 3.45\left(\mathrm{H}_{1-\mathrm{C}}, 90 \times 4 \mathrm{H}\right.$, br s$)$, $3.32\left(\mathrm{H}_{\mathrm{d}-\mathrm{C}}, 60 \times 2 \mathrm{H}\right.$, br s $), 2.34\left(\mathrm{H}_{\mathrm{d}-\mathrm{A}}, \mathrm{H}_{1-\mathrm{A}}\right.$ and $\mathrm{H}_{\mathrm{t}-\mathrm{B}},(60 \times 6+90 \times 6+3) \mathrm{H}$, br s $), 1.88-1.13$ 
$\left(\mathrm{H}_{\mathrm{d}-\mathrm{D}}, \mathrm{H}_{\mathrm{d}-\mathrm{E}}, \mathrm{H}_{\mathrm{d}-\mathrm{F}}, \mathrm{H}_{\mathrm{d}-\mathrm{G}}, \mathrm{H}_{\mathrm{d}-\mathrm{I}}\right.$ and $\mathrm{H}_{1-\mathrm{D}},(60 \times 20+90 \times 4) \mathrm{H}$, br m), $0.89\left(\mathrm{H}_{\mathrm{d}-\mathrm{H}}, \mathrm{H}_{\mathrm{d}-\mathrm{J}}\right.$ and $\mathrm{H}_{1-\mathrm{E}}$, $(60 \times 12+90 \times 6) \mathrm{H}$, br s), small peaks originated from end-group were observed in 7.87$7.07 \mathrm{ppm}\left(\mathrm{H}_{\mathrm{t}-\mathrm{C}}, 4 \mathrm{H}, \mathrm{m}\right) ; \mathrm{GPC}\left(\mathrm{CHCl}_{3}, \mathrm{~g} / \mathrm{mol}\right): M_{\mathrm{n}}=3.23 \times 10^{5}, M_{\mathrm{w}} / M_{\mathrm{n}}=1.15$.

Synthesis of polymer $3 \mathbf{d}(\mathbf{8 0} / \mathbf{1 2 0})$ : A THF solution of $o$-TolNiCl( $\left(\mathrm{PMe}_{3}\right)_{2}(6.70 \mathrm{mmol} / \mathrm{L}$, $641 \mu \mathrm{L}, 4.30 \mu \mathrm{mol})$ was added to $20 \mathrm{~mL}$ THF solution of Qd (151.6 mg, $344 \mu \mathrm{mol})$ and Q3 (155.0 mg, $516 \mu \mathrm{mol})$ with vigorous stirring. After $12 \mathrm{~h}, \mathrm{NaBH}_{4}(34.16 \mathrm{mg}, 903$ $\mu \mathrm{mol})$ was added to the reaction mixture and stirred for $1 \mathrm{~h}$. Water $(50 \mathrm{~mL})$ was added to the solution and extracted with $\mathrm{CH}_{2} \mathrm{Cl}_{2}(50 \mathrm{ml})$. The organic extract was washed with brine $(50 \mathrm{~mL})$, dried over $\mathrm{Na}_{2} \mathrm{SO}_{4}$, and the solvent was evaporated. The residue was subjected to preparative GPC to give $\mathbf{3 d}(\mathbf{8 0} / \mathbf{1 2 0})$ as a beige solid $(294.5 \mathrm{mg}, 96 \%) .{ }^{1} \mathrm{H}$ $\operatorname{NMR}\left(\mathrm{CDCl}_{3}\right) \delta 4.58\left(\mathrm{H}_{\mathrm{d}-\mathrm{B}}\right.$ and $\mathrm{H}_{1-\mathrm{B}},(80 \times 4+120 \times 4) \mathrm{H}$, br s), $3.45\left(\mathrm{H}_{1-\mathrm{C}}, 120 \times 4 \mathrm{H}\right.$, br s), $3.32\left(\mathrm{H}_{\mathrm{d}-\mathrm{C}}, 80 \times 2 \mathrm{H}\right.$, br s), $2.35\left(\mathrm{H}_{\mathrm{d}-\mathrm{A}}, \mathrm{H}_{1-\mathrm{A}}\right.$ and $\mathrm{H}_{\mathrm{t}-\mathrm{B}},(80 \times 6+120 \times 6+3) \mathrm{H}$, br s $), 1.72-1.15$ $\left(\mathrm{H}_{\mathrm{d}-\mathrm{D}}, \mathrm{H}_{\mathrm{d}-\mathrm{E}}, \mathrm{H}_{\mathrm{d}-\mathrm{F}}, \mathrm{H}_{\mathrm{d}-\mathrm{G}}, \mathrm{H}_{\mathrm{d}-\mathrm{I}}\right.$ and $\mathrm{H}_{1-\mathrm{D}},(80 \times 20+120 \times 4) \mathrm{H}$, br m), $0.89\left(\mathrm{H}_{\mathrm{d}-\mathrm{H}}, \mathrm{H}_{\mathrm{d}-\mathrm{J}}\right.$ and $\mathrm{H}_{1-\mathrm{E}}$, $(80 \times 12+120 \times 6) \mathrm{H}$, br s), small peaks originated from end-group were observed in 9.94 $\left(\mathrm{H}_{\mathrm{t}-\mathrm{A}}, 1 \mathrm{H}, \mathrm{s}\right)$ and 7.87-7.09ppm $\left(\mathrm{H}_{\mathrm{t}-\mathrm{C}}, 4 \mathrm{H}, \mathrm{m}\right) ; \mathrm{GPC}\left(\mathrm{CHCl}_{3}, \mathrm{~g} / \mathrm{mol}\right): M_{\mathrm{n}}=5.22 \times 10^{4}$, $M_{\mathrm{w}} / M_{\mathrm{n}}=1.11$.

Synthesis of polymer 3d(100/150): A THF solution of $o$-TolNiCl( $\left(\mathrm{PMe}_{3}\right)_{2}(12.34$ $\mathrm{mmol} / \mathrm{L}, 20.3 \mu \mathrm{L}, 0.250 \mu \mathrm{mol})$ was added to $3.0 \mathrm{~mL}$ THF solution of Qd (11.02 $\mathrm{mg}$, $25.0 \mu \mathrm{mol})$ and $\mathbf{Q 3}(11.27 \mathrm{mg}, 37.5 \mu \mathrm{mol})$ with vigorous stirring. After $27 \mathrm{~h}, \mathrm{NaBH}_{4}$ (1.99 $\mathrm{mg}, 52.5 \mu \mathrm{mol})$ was added to the reaction mixture and stirred for $1 \mathrm{~h}$. Water $(15$ $\mathrm{mL}$ ) was added to the solution and extracted with $\mathrm{CH}_{2} \mathrm{Cl}_{2}(15 \mathrm{ml})$. The organic extract was washed with brine $(15 \mathrm{~mL})$, dried over $\mathrm{Na}_{2} \mathrm{SO}_{4}$, and the solvent was evaporated. The residue was subjected to preparative GPC to give $\mathbf{3 d}(\mathbf{1 0 0} / \mathbf{1 5 0})$ as a beige solid (18.5 mg, 83\%). ${ }^{1} \mathrm{H}$ NMR $\left(\mathrm{CDCl}_{3}\right) \delta 4.66\left(\mathrm{H}_{\mathrm{d}-\mathrm{B}}\right.$ and $\mathrm{H}_{1-\mathrm{B}},(100 \times 4+150 \times 4) \mathrm{H}$, br s $), 3.45$ $\left(\mathrm{H}_{1-\mathrm{C}}, 150 \times 4 \mathrm{H}\right.$, br s $), 3.32\left(\mathrm{H}_{\mathrm{d}-\mathrm{C}}, 100 \times 2 \mathrm{H}\right.$, br s $), 2.34\left(\mathrm{H}_{\mathrm{d}-\mathrm{A}}, \mathrm{H}_{1-\mathrm{A}}\right.$ and $\mathrm{H}_{\mathrm{t}-\mathrm{B}}$, $(100 \times 6+150 \times 6+3) \mathrm{H}, \quad$ br $\mathrm{s}), \quad 1.68-1.17 \quad\left(\mathrm{H}_{\mathrm{d}-\mathrm{D}}, \mathrm{H}_{\mathrm{d}-\mathrm{E}}, \mathrm{H}_{\mathrm{d}-\mathrm{F}}, \mathrm{H}_{\mathrm{d}-\mathrm{G}}, \mathrm{H}_{\mathrm{d}-\mathrm{I}}\right.$ and $\mathrm{H}_{1-\mathrm{D}}$, $(100 \times 20+150 \times 4) \mathrm{H}$, br m), $0.89\left(\mathrm{H}_{\mathrm{d}-\mathrm{H}}, \mathrm{H}_{\mathrm{d}-\mathrm{J}}\right.$ and $\mathrm{H}_{1-\mathrm{E}},(100 \times 12+150 \times 6) \mathrm{H}$, br s), small peaks originated from end-group were observed in $10.01\left(\mathrm{H}_{\mathrm{t}-\mathrm{A}}, 1 \mathrm{H}, \mathrm{s}\right)$ and $7.87-$ 7.08ppm $\left(\mathrm{H}_{\mathrm{t}-\mathrm{C}}, 4 \mathrm{H}, \mathrm{m}\right) ; \mathrm{GPC}\left(\mathrm{CHCl}_{3}, \mathrm{~g} / \mathrm{mol}\right): M_{\mathrm{n}}=1.03 \times 10^{5}, M_{\mathrm{w}} / M_{\mathrm{n}}=1.11$. 
Synthesis of polymer 3d(120/180): A THF solution of $o$ - $\mathrm{TolNiCl}\left(\mathrm{PMe}_{3}\right)_{2}(12.34$ $\mathrm{mmol} / \mathrm{L}, 16.9 \mu \mathrm{L}, 0.208 \mu \mathrm{mol})$ was added to $3.0 \mathrm{~mL}$ THF solution of Qd (11.02 $\mathrm{mg}$, $25.0 \mu \mathrm{mol})$ and $\mathbf{Q 3}(11.27 \mathrm{mg}, 37.5 \mu \mathrm{mol})$ with vigorous stirring. After $27 \mathrm{~h}, \mathrm{NaBH}_{4}$ $(1.66 \mathrm{mg}, 43.8 \mu \mathrm{mol})$ was added to the reaction mixture and stirred for $1 \mathrm{~h}$. Water $(15$ $\mathrm{mL})$ was added to the solution and extracted with $\mathrm{CH}_{2} \mathrm{Cl}_{2}(15 \mathrm{ml})$. The organic extract was washed with brine $(15 \mathrm{~mL})$, dried over $\mathrm{Na}_{2} \mathrm{SO}_{4}$, and the solvent was evaporated. The residue was subjected to preparative GPC to give $\mathbf{3 d ( 1 2 0 / 1 8 0 )}$ as a beige solid (20.6 mg, 92\%). ${ }^{1} \mathrm{H}$ NMR $\left(\mathrm{CDCl}_{3}\right) \delta 4.70\left(\mathrm{H}_{\mathrm{d}-\mathrm{B}}\right.$ and $\mathrm{H}_{1-\mathrm{B}},(120 \times 4+180 \times 4) \mathrm{H}$, br s$), 3.45$ $\left(\mathrm{H}_{1-\mathrm{C}}, 180 \times 4 \mathrm{H}\right.$, br s$), 3.31\left(\mathrm{H}_{\mathrm{d}-\mathrm{C}}, 120 \times 2 \mathrm{H}\right.$, br s$), 2.34\left(\mathrm{H}_{\mathrm{d}-\mathrm{A}}, \mathrm{H}_{1-\mathrm{A}}\right.$ and $\mathrm{H}_{\mathrm{t}-\mathrm{B}}$, $(120 \times 6+180 \times 6+3) \mathrm{H}$, br s), $1.66-1.21 \quad\left(\mathrm{H}_{\mathrm{d}-\mathrm{D}}, \mathrm{H}_{\mathrm{d}-\mathrm{E}}, \mathrm{H}_{\mathrm{d}-\mathrm{F}}, \mathrm{H}_{\mathrm{d}-\mathrm{G}}, \mathrm{H}_{\mathrm{d}-\mathrm{I}}\right.$ and $\mathrm{H}_{1-\mathrm{D}}$, $(120 \times 20+180 \times 4) \mathrm{H}$, br m), $0.88\left(\mathrm{H}_{\mathrm{d}-\mathrm{H}}, \mathrm{H}_{\mathrm{d}-\mathrm{J}}\right.$ and $\mathrm{H}_{1-\mathrm{E}},(120 \times 12+180 \times 6) \mathrm{H}$, br s), small peaks originated from end-group were observed in $10.02\left(\mathrm{H}_{\mathrm{t}-\mathrm{A}}, 1 \mathrm{H}, \mathrm{s}\right)$ and $7.86-$ 7.08ppm $\left(\mathrm{H}_{\mathrm{t}-\mathrm{C}}, 4 \mathrm{H}, \mathrm{m}\right) ; \mathrm{GPC}\left(\mathrm{CHCl}_{3}, \mathrm{~g} / \mathrm{mol}\right): M_{\mathrm{n}}=1.20 \times 10^{5}, M_{\mathrm{w}} / M_{\mathrm{n}}=1.12$.

Synthesis of polymer 4: A THF solution of $o$-TolNiCl( $\left(\mathrm{PMe}_{3}\right)_{2}(12.34 \mathrm{mM}, 180 \mu \mathrm{L}$, $2.22 \mu \mathrm{mol})$ was added to the solution of monomer Qd $(391.2 \mathrm{mg}, 887.8 \mu \mathrm{mol}), \mathbf{Q 3}$ (400.0 mg, $1.332 \mathrm{mmol})$ and Qp $(9.64 \mathrm{mg}, 22.2 \mu \mathrm{mol})$ in THF $(50 \mathrm{~mL})$. The mixture was stirred for $16 \mathrm{~h}$ at room temperature. To the reaction mixture was added $\mathrm{NaBH}_{4}$ (17.63 mg, $466.0 \mu \mathrm{mol}$ ), and the mixture was stirred for $1 \mathrm{~h}$. The mixture was poured into vigorously stirred methanol $(300 \mathrm{~mL})$, and precipitated polymer was collected by filtration. After drying in vacuo, fibriform polymer was obtained.

Reduction of phosphine sulfide: A mixture of the obtained polymer $(20.7 \mu \mathrm{mol} P)$ and $\mathrm{P}\left(\mathrm{NMe}_{2}\right)_{3}(147.2 \mu \mathrm{L}, 810 \mu \mathrm{mol})$ in toluene $(11 \mathrm{~mL})$ was stirred at $110{ }^{\circ} \mathrm{C}$ for $19 \mathrm{~h}$. The mixture was poured into vigorously stirred $\mathrm{MeOH}(300 \mathrm{~mL})$. Precipitated material was collected by filtration to give 4 as fibriform solid $(715.2 \mathrm{mg}, 89 \%) .{ }^{1} \mathrm{H}$ NMR $\left(\mathrm{CDCl}_{3}\right) \delta$ $4.62\left(\mathrm{H}_{\mathrm{d}-\mathrm{B}}\right.$ and $\mathrm{H}_{1-\mathrm{B}},(400 \times 4+600 \times 4) \mathrm{H}$, br s), $3.46\left(\mathrm{H}_{1-\mathrm{C}}, 400 \times 4 \mathrm{H}\right.$, br s$), 3.32\left(\mathrm{H}_{\mathrm{d}-\mathrm{C}}\right.$, $400 \times 2 \mathrm{H}$, br s), $2.36\left(\mathrm{H}_{\mathrm{d}-\mathrm{A}}, \mathrm{H}_{1-\mathrm{A}}\right.$ and $\mathrm{H}_{\mathrm{t}-\mathrm{B}},(400 \times 6+600 \times 6+3) \mathrm{H}$, br s $), 2.11-1.17\left(\mathrm{H}_{\mathrm{d}-\mathrm{D}}\right.$, $\mathrm{H}_{\mathrm{d}-\mathrm{E}}, \mathrm{H}_{\mathrm{d}-\mathrm{F}}, \mathrm{H}_{\mathrm{d}-\mathrm{G}}, \mathrm{H}_{\mathrm{d}-\mathrm{I}}$ and $\mathrm{H}_{1-\mathrm{D}},(400 \times 20+600 \times 4) \mathrm{H}$, br $\left.\mathrm{m}\right), 0.89\left(\mathrm{H}_{\mathrm{d}-\mathrm{H}}, \mathrm{H}_{\mathrm{d}-\mathrm{J}}\right.$ and $\mathrm{H}_{1-\mathrm{E}}$, $(400 \times 12+600 \times 6) \mathrm{H}$, br s), small peaks originated from end-group and $\mathbf{Q}_{\mathbf{p}}$ units were observed in 7.88-7.09 ppm; ${ }^{31} \mathrm{P}$ NMR $\left(\mathrm{CDCl}_{3}\right) \delta-15.0 ; \mathrm{GPC}\left(\mathrm{CHCl}_{3}, \mathrm{~g} / \mathrm{mol}\right): M_{\mathrm{n}}=5.12$ 
$\times 10^{5}, M_{\mathrm{w}} / M_{\mathrm{n}}=1.11$

Scheme S6. Synthesis of polymer 4.
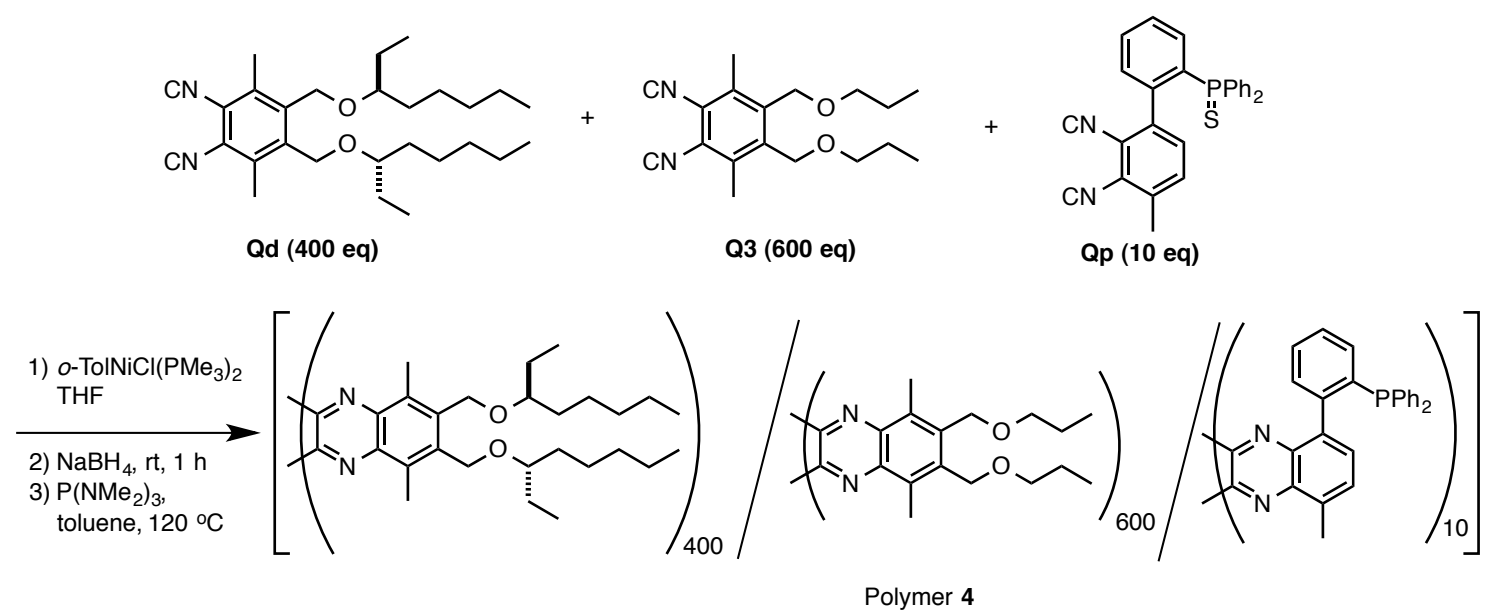

\section{Use of Polymer 4 as a Chiral Ligand in the Asymmetric Suzuki-Miyaura Cross-Coupling Reaction}

\section{Asymmetric Suzuki-Miyaura cross-coupling reaction using polymer 4 as a ligand}

in PhH: The solution of polymer ligand 4 (297.75 mg, $8.25 \mu \mathrm{mol}$ phosphorus atom) in $\mathrm{PhH}(2 \mathrm{~mL})$ was stirred for $12 \mathrm{~h}$ for $60{ }^{\circ} \mathrm{C}$. $\mathrm{Pd}_{2} \mathrm{dba}_{3}(2.56 \mathrm{mM}$ in toluene, $805 \mu \mathrm{L}, 2.06$ $\mu \mathrm{mol})$ was added to the solution. The solvent was evaporated in vacuo. To polymer ligand 4 (297.75 mg, $8.25 \mu \mathrm{mol}$ phosphorus atom) was added $\mathrm{Pd}_{2} \mathrm{dba}_{3}(2.56 \mathrm{mM}$ in toluene, $805 \mu \mathrm{L}, 2.06 \mu \mathrm{mol})$. The solvent was evaporated in vacuo. To the mixture were added $\mathrm{K}_{3} \mathrm{PO}_{4}(26.27 \mathrm{mg}, 123.8 \mu \mathrm{mol})$, aryl bromide 5 (13.0 mg, $\left.41.3 \mu \mathrm{mol}\right)$, boronic acid 6 (15.35 mg, $82.5 \mu \mathrm{mol}), \mathrm{H}_{2} \mathrm{O}(33 \mu \mathrm{L})$, and $\mathrm{PhH}(2.5 \mathrm{~mL})$. The mixture was stirred at $\mathrm{rt}$ for $48 \mathrm{~h}$. Addition of MeCN (10 mL) resulted in precipitation of polymer ligand 4. The suspension was passed through a pad of Celite using MeCN as an eluent. The filtrate was purified by preparative GPC. The corresponding product $(+)-(R)$-form was isolated in $75 \%$ yield. ${ }^{1} \mathrm{H}$ NMR $\left(\mathrm{CDCl}_{3}\right) \delta 8.16(1 \mathrm{H}, \mathrm{dd}, J=12.0,8.8 \mathrm{~Hz}), 8.09(1 \mathrm{H}, \mathrm{d}$, $J=8.4 \mathrm{~Hz}), 8.02(1 \mathrm{H}, \mathrm{dd}, J=8.4,3.6 \mathrm{~Hz}), 7.94(1 \mathrm{H}, \mathrm{d}, J=8.0 \mathrm{~Hz}), 7.53(1 \mathrm{H}, \mathrm{ddd}, J=$ 8.0, 6.4, $1.2 \mathrm{~Hz}), 7.48(1 \mathrm{H}, \mathrm{ddd}, J=8.0,6.8,1.2 \mathrm{~Hz}), 7.46(1 \mathrm{H}, \mathrm{dd}, J=5.2,0.8 \mathrm{~Hz})$, $7.37(1 \mathrm{H}, \mathrm{d}, J=7.2 \mathrm{~Hz}), 7.22-7.29(2 \mathrm{H}, \mathrm{m}), 7.20(1 \mathrm{H}, \mathrm{d}, J=7.6 \mathrm{~Hz}), 7.10(1 \mathrm{H}, \mathrm{dd}, J=$ 8.4, $0.4 \mathrm{~Hz}), 3.27(3 \mathrm{H}, \mathrm{d}, J=12.0 \mathrm{~Hz}), 3.24(3 \mathrm{H}, \mathrm{d}, J=11.6 \mathrm{~Hz}), 2.82(3 \mathrm{H}, \mathrm{s}) ;{ }^{13} \mathrm{C}$ 
$\operatorname{NMR}\left(\mathrm{CDCl}_{3}\right) \delta 144.1\left(J_{\mathrm{CP}}=10.1 \mathrm{~Hz}\right), 134.9,134.6,134.0\left(J_{\mathrm{CP}}=5.4 \mathrm{~Hz}\right), 133.4\left(J_{\mathrm{CP}}=\right.$ $16.3 \mathrm{~Hz}), 133.2,133.2,128.6\left(J_{\mathrm{CP}}=10.0 \mathrm{~Hz}\right), 128.2\left(J_{\mathrm{CP}}=1.5 \mathrm{~Hz}\right), 127.9,127.9\left(J_{\mathrm{CP}}=\right.$ $4.6 \mathrm{~Hz}), 127.7,127.6,127.2,126.7\left(J_{\mathrm{CP}}=1.5 \mathrm{~Hz}\right), 125.8,125.5,125.4,125.3\left(J_{\mathrm{CP}}=\right.$ $188.1 \mathrm{~Hz}), 124.2,52.3\left(J_{\mathrm{CP}}=20.9 \mathrm{~Hz}\right), 52.2\left(J_{\mathrm{CP}}=21.7 \mathrm{~Hz}\right), 19.7 ;{ }^{31} \mathrm{P} \mathrm{NMR}\left(\mathrm{CDCl}_{3}\right) \delta$ 21.3; IR (ATR) 3057, 2947, 2846, 1616, 1589, 1558, 1512, 1365, 1242, 1180, 1022, 879, 819, 758, $688 \mathrm{~cm}^{-1}$; HRMS (EI) $\mathrm{m} / z$ calcd for $\mathrm{C}_{23} \mathrm{H}_{21} \mathrm{O}_{3} \mathrm{P}\left(\mathrm{M}^{+}\right): 376.1224$, found: $376.1226 ;[\alpha]_{\mathrm{D}}^{26.0}+41.2\left[\mathrm{c} 1.410, \mathrm{CH}_{2} \mathrm{Cl}_{2}, 98 \% \text { ee }(R)\right]^{11}$; UV/Vis $\left(2.25 \times 10^{-4} \mathrm{M}\right.$ in $\mathrm{MeCN}): \lambda_{\max }(\varepsilon)=197.0\left(4.42 \times 10^{3}\right), 226.0\left(5.27 \times 10^{3}\right), 290.5\left(6.93 \times 10^{2}\right) \mathrm{nm} ; \mathrm{CD}$ $\left(2.25 \times 10^{-4} \mathrm{M}\right.$ in $\left.\mathrm{MeCN}\right): \lambda_{\max }(\Delta \varepsilon)=220.0\left(-5.83 \times 10^{2}\right), 232.0\left(+1.27 \times 10^{3}\right), 285.0$ $(-2.25 \times 10) \mathrm{nm}$. The enantiomeric excess of the product was determined to be $91 \%$ by HPLC with CHIRALCEL ${ }^{\circledR}$ OZ-H (Eluent: Hexane/2-PrOH $=80 / 20$, Flow rate: 0.6 $\mathrm{mL} / \mathrm{min}$, Retention time: $t_{\mathrm{R}}$ of $(-)$-isomer $=13.3 \mathrm{~min}, t_{\mathrm{R}}$ of $(+)$-isomer $\left.=16.6 \mathrm{~min}\right)$.

Scheme S7. Asymmetric Suzuki-Miyaura cross-coupling reaction using polymer 4 as a ligand in $\mathrm{PhH}$.

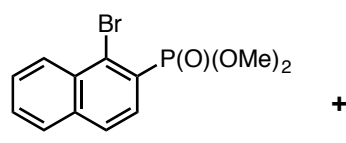

5

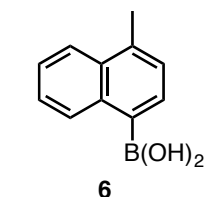

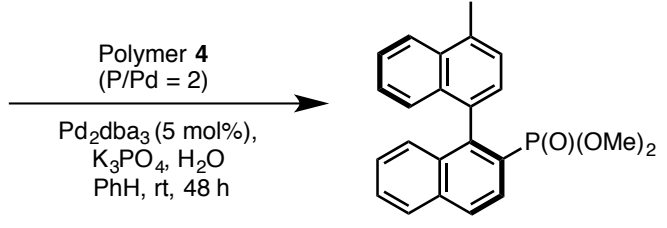

$(R)-7$

\section{Asymmetric Suzuki-Miyaura cross-coupling reaction using polymer 4 as a ligand} in $\mathbf{P h C F}_{3}$ : The solution of polymer ligand $\mathbf{4}$ (297.75 $\mathrm{mg}, 8.25 \mu \mathrm{mol}$ phosphorus atom) in $\mathrm{PhCF}_{3}(2 \mathrm{~mL})$ was stirred for $12 \mathrm{~h}$ for $60{ }^{\circ} \mathrm{C} . \mathrm{Pd}_{2} \mathrm{dba}_{3}(2.56 \mathrm{mM}$ in toluene, $805 \mu \mathrm{L}$, $2.06 \mu \mathrm{mol})$ was added to the solution. The solvent was evaporated in vacuo. To polymer ligand 4 (297.75 mg, $8.25 \mu \mathrm{mol}$ phosphorus atom) was added $\mathrm{Pd}_{2} \mathrm{dba}_{3}(2.56 \mathrm{mM}$ in toluene, $805 \mu \mathrm{L}, 2.06 \mu \mathrm{mol})$. The solvent was evaporated in vacuo. To the mixture were added $\mathrm{K}_{3} \mathrm{PO}_{4}(26.27 \mathrm{mg}, 123.8 \mu \mathrm{mol})$, aryl bromide 5 (13.0 mg, $\left.41.3 \mu \mathrm{mol}\right)$, boronic acid $6(15.35 \mathrm{mg}, 82.5 \mu \mathrm{mol}), \mathrm{H}_{2} \mathrm{O}(33 \mu \mathrm{L})$, and $\mathrm{PhCF}_{3}(2.5 \mathrm{~mL})$. The mixture was stirred at $\mathrm{rt}$ for $48 \mathrm{~h}$. Addition of $\mathrm{MeCN}(10 \mathrm{~mL})$ resulted in precipitation of polymer ligand 4. The suspension was passed through a pad of Celite using $\mathrm{MeCN}$ as an eluent. The filtrate was purified by preparative GPC. The corresponding product $(-)-(S)$-form was isolated in $48 \%$ yield. The enantiomeric excess of the product was determined to be 
95\% by HPLC with CHIRALCEL ${ }^{\circledR}$ OZ-H (Eluent: Hexane/2-PrOH $=80 / 20$, Flow rate: $0.6 \mathrm{~mL} / \mathrm{min}$, Retention time: $t_{\mathrm{R}}$ of $(-)$-isomer $=13.3 \mathrm{~min}, t_{\mathrm{R}}$ of $(+)$-isomer $\left.=16.6 \mathrm{~min}\right)$.

Scheme S8. Asymmetric Suzuki-Miyaura cross-coupling reaction using polymer 4 as a ligand in $\mathrm{PhCF}_{3}$.

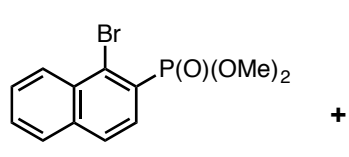

5<smiles>Cc1ccc(Br)c2ccccc12</smiles>

6

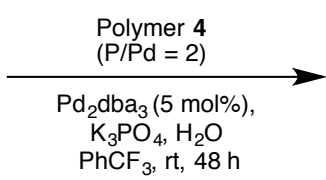

$\mathrm{K}_{3} \mathrm{PO}_{4}, \mathrm{H}_{2} \mathrm{O}$

$\mathrm{PhCF}_{3}, \mathrm{rt}, 48 \mathrm{~h}$

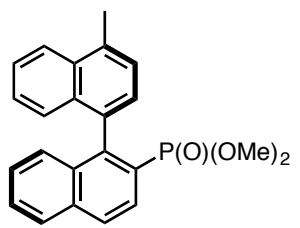

$(S)-7$ 


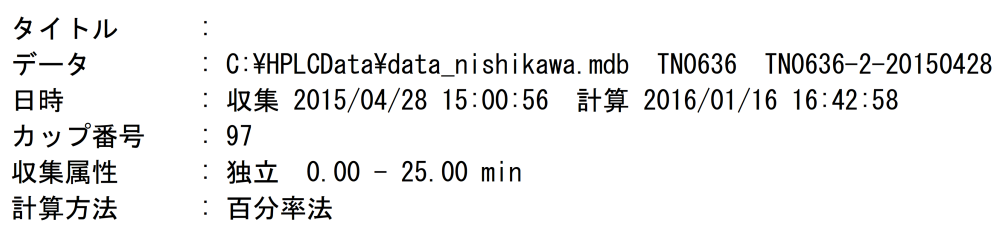

[mV]

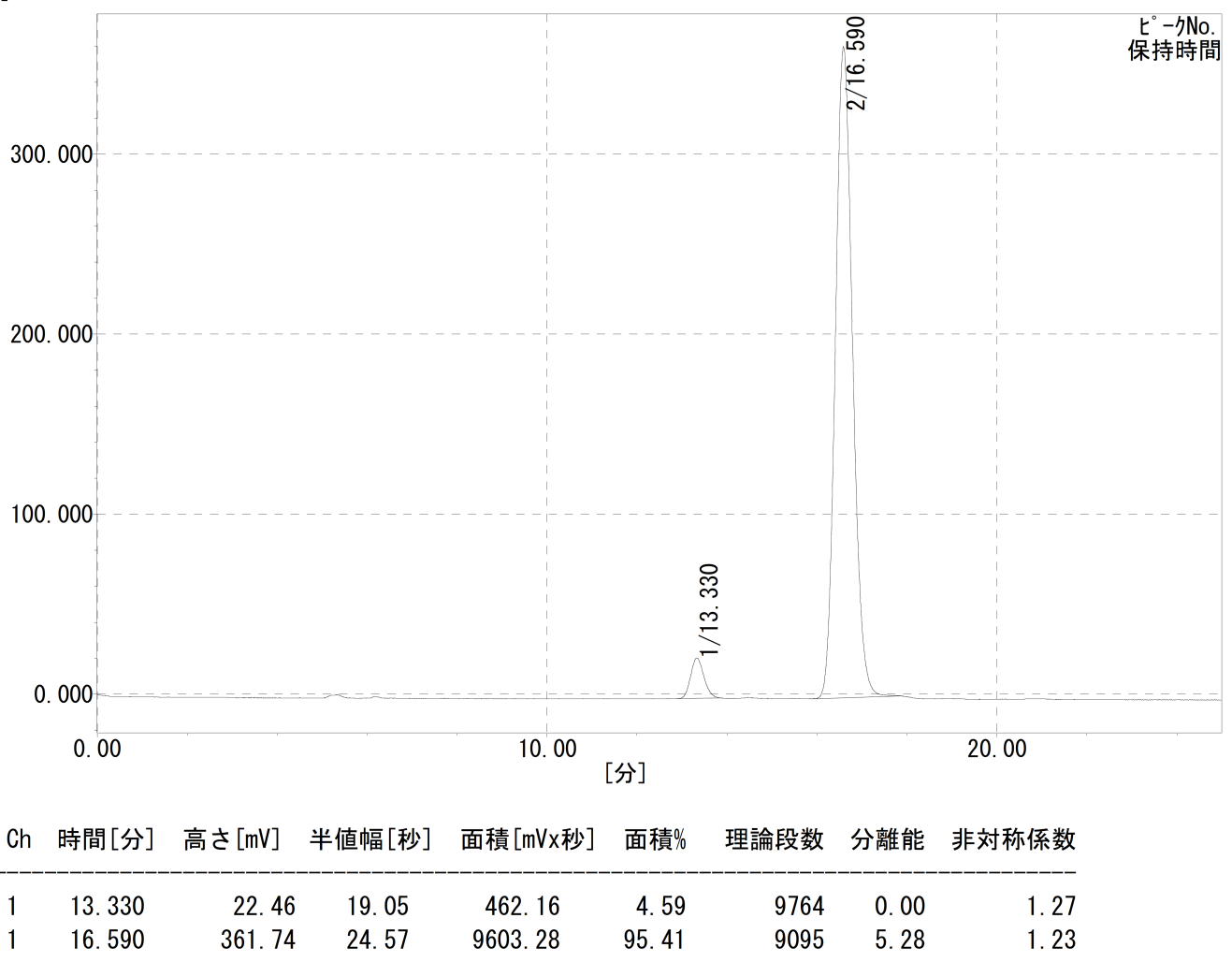

CH.1 Peak Not Found.

Figure S6. HPLC trace of the product of the asymmetric Suzuki-Miyaura Cross coupling reaction in $\mathrm{PhH}$ (Enantiomeric excess was found to be $91 \%(R)$ ). 


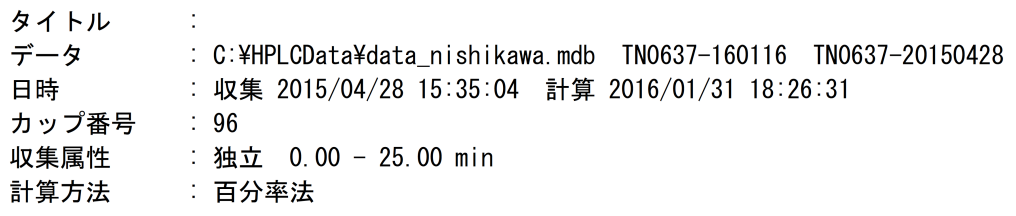

$[\mathrm{mV}]$

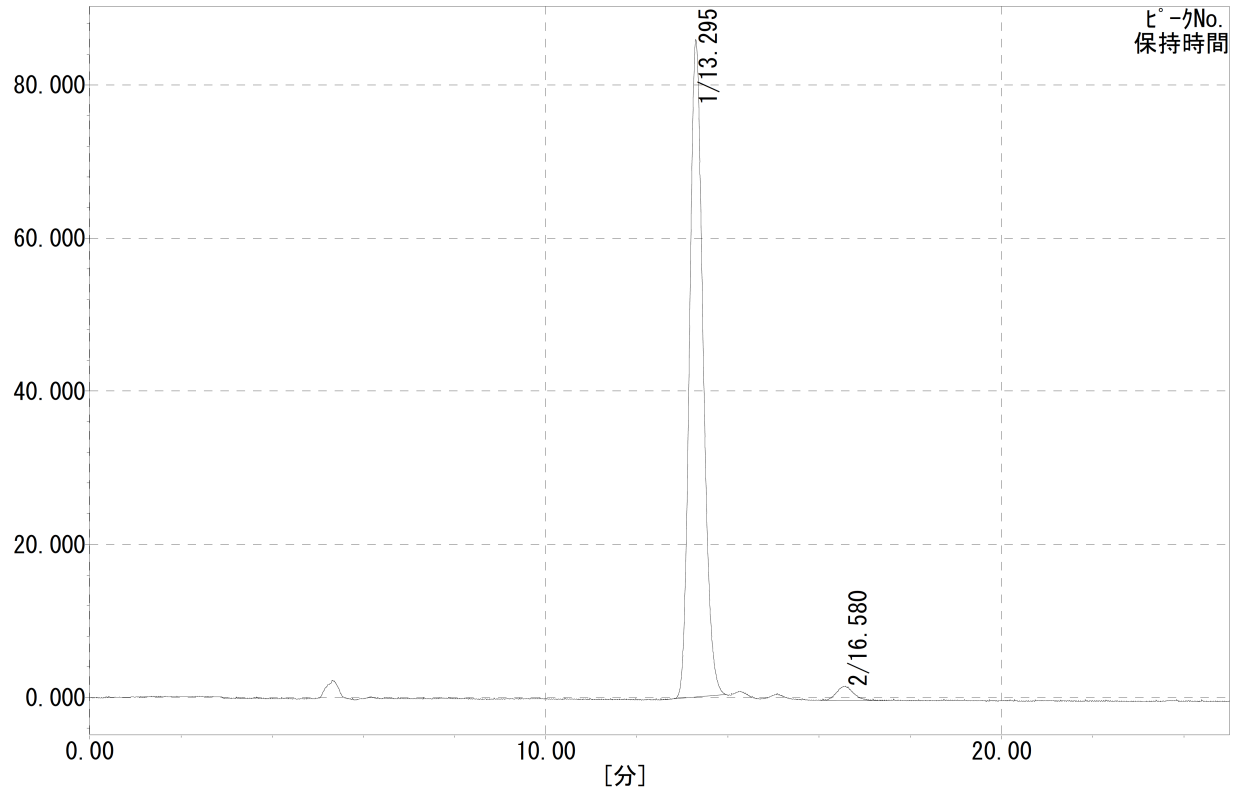

No. Ch 時間[分] 高さ [mV] 半値幅[秒] 面積 $[\mathrm{mVx}$ 秒] 面積\% 理論段数 分離能 非対称係数

\begin{tabular}{|c|c|c|c|c|c|c|c|c|c|}
\hline 1 & 1 & 13. 295 & 85.85 & 19. 10 & 1779. 29 & 97.38 & 9660 & 0.00 & 1. 29 \\
\hline 2 & 1 & 16. 580 & 1.85 & 23. 42 & 47.96 & 2. 62 & 9998 & 5. 46 & 1. 69 \\
\hline
\end{tabular}

CH.1 Peak Not Found.

Figure S7. HPLC trace of the product of the asymmetric Suzuki-Miyaura Cross coupling reaction in $\mathrm{PhCF}_{3}$ (Enantiomeric excess was found to be 95\% $(S)$ ). 


\section{Chiroptical Properties of Polymer Solutions}

Table S1. Dissymmetry factors $g_{\text {abs }}$ of polymers for Table 1 .

\begin{tabular}{ccccc}
\hline \multirow{2}{*}{ Polymer } & \multicolumn{4}{c}{$g_{\text {abs }}$ at $366.0 \mathrm{~nm} / 10^{-3}$} \\
\cline { 2 - 5 } & $\mathrm{PhH}$ & $\mathrm{PhCH}_{3}$ & $\mathrm{PhOCH}_{3}$ & $\mathrm{PhCF}_{3}$ \\
\hline $\mathbf{1 a}(\mathbf{1 0 0})$ & -2.10 & -2.27 & -2.33 & -2.30 \\
$\mathbf{1 b}(\mathbf{1 0 0})$ & +1.54 & +1.56 & +1.49 & +1.51 \\
$\mathbf{1 c}(\mathbf{1 0 0})$ & -1.63 & -1.85 & -1.85 & -1.77 \\
$\mathbf{1 d}(\mathbf{1 0 0})$ & -1.68 & -1.92 & -1.65 & -1.80 \\
$\mathbf{2 a ( 5 / 1 0 0 )}$ & -0.39 & -0.23 & -0.36 & -0.31 \\
$\mathbf{2 b}(\mathbf{5} / \mathbf{1 0 0})$ & +0.24 & +0.25 & +0.19 & +0.21 \\
$\mathbf{2 c ( 5 / 1 0 0 )}$ & -0.54 & -0.39 & -0.20 & +0.03 \\
$\mathbf{2 d ( 5 / 1 0 0 )}$ & +0.11 & +0.15 & +0.32 & +0.54 \\
$\mathbf{3 a ( 5 / 1 0 0 )}$ & -0.62 & -0.49 & -0.75 & -0.49 \\
$\mathbf{3 b}(\mathbf{5 / 1 0 0})$ & +0.30 & +0.25 & +0.18 & +0.23 \\
$\mathbf{3 c ( 5 / 1 0 0 )}$ & -0.74 & -0.62 & -0.55 & -0.19 \\
$\mathbf{3 d}(\mathbf{5} / \mathbf{1 0 0})$ & -0.17 & -0.10 & +0.17 & +0.41 \\
\hline
\end{tabular}


Table S2. Dissymmetry factors $g_{\text {abs }}$ of polymers for Figure 1.

\begin{tabular}{ccccc}
\hline \multirow{2}{*}{ Polymer } & \multicolumn{4}{c}{$g_{\text {abs }}$ at $366.0 \mathrm{~nm} / 10^{-3}$} \\
\cline { 2 - 5 } & $\mathrm{PhH}$ & $\mathrm{PhCH}_{3}$ & $\mathrm{PhOCH}_{3}$ & $\mathrm{PhCF}_{3}$ \\
\hline $\mathbf{3 d}(\mathbf{2 . 5 / 9 7 . 5 )}$ & -0.07 & -0.02 & +0.12 & +0.26 \\
$\mathbf{3 d}(\mathbf{5 / 9 5})$ & -0.16 & -0.06 & +0.23 & +0.43 \\
$\mathbf{3 d}(\mathbf{7 . 5 / 9 2 . 5 )}$ & -0.26 & -0.12 & +0.34 & +0.63 \\
$\mathbf{3 d}(\mathbf{1 0} / \mathbf{9 0})$ & -0.36 & -0.14 & +0.43 & +0.79 \\
$\mathbf{3 d}(\mathbf{1 5 / 8 0})$ & -0.59 & -0.29 & +0.61 & +1.04 \\
$\mathbf{3 d}(\mathbf{2 0} / \mathbf{8 0})$ & -0.90 & -0.54 & +0.76 & +1.34 \\
$\mathbf{3 d ( 3 0 / 7 0 )}$ & -1.19 & -0.86 & +0.87 & +1.49 \\
$\mathbf{3 d}(\mathbf{4 0} / \mathbf{6 0})$ & -1.38 & -1.13 & +0.89 & +1.55 \\
$\mathbf{3 d}(\mathbf{6 0} / \mathbf{4 0})$ & -1.66 & -1.78 & +0.51 & +1.42 \\
$\mathbf{3 d}(\mathbf{8 0} / \mathbf{2 0})$ & -1.68 & -1.94 & -0.59 & -0.08 \\
\hline
\end{tabular}

Table S3. Dissymmetry factors $g_{\text {abs }}$ of polymers for Figure 2 .

\begin{tabular}{ccc}
\hline \multirow{2}{*}{ Polymer } & \multicolumn{2}{c}{$g_{\text {abs }}$ at $366.0 \mathrm{~nm} / 10^{-3}$} \\
\cline { 2 - 3 } & $\mathrm{PhH}$ & $\mathrm{PhCF}_{3}$ \\
\hline $\mathbf{3 d}(\mathbf{2 0} / \mathbf{3 0})$ & -0.85 & +1.03 \\
$\mathbf{3 d}(\mathbf{6 0} / \mathbf{9 0})$ & -1.38 & +1.55 \\
$\mathbf{3 d}(\mathbf{8 0} / \mathbf{1 2 0})$ & -1.68 & +2.00 \\
$\mathbf{3 d}(\mathbf{1 0 0} / \mathbf{1 5 0})$ & -1.68 & +2.04 \\
$\mathbf{3 d}(\mathbf{1 2 0} / \mathbf{1 8 0})$ & -1.72 & +2.04 \\
\hline
\end{tabular}




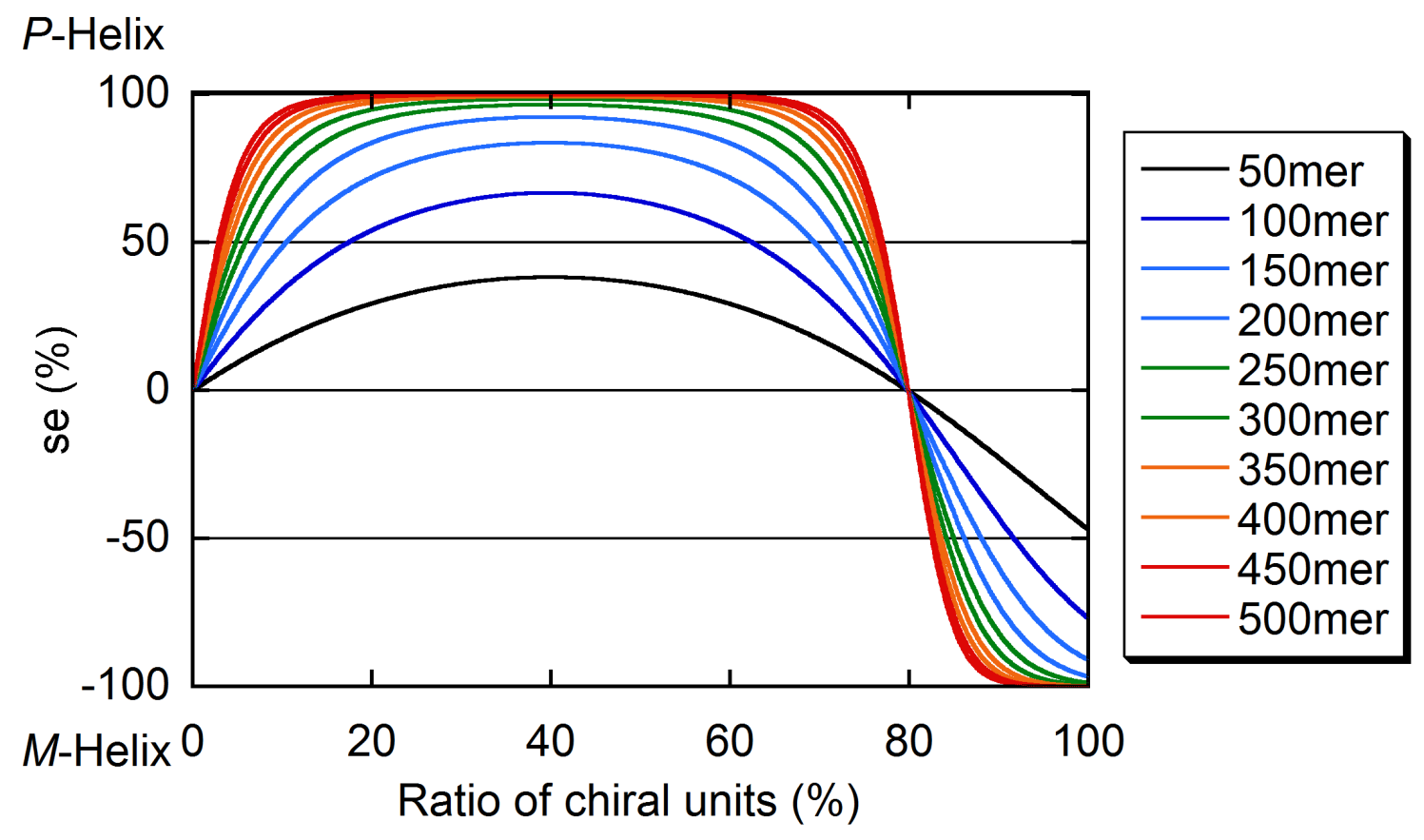

Figure S8. Simulated correlation between the ratio of chiral units and the se of $\mathbf{3 d}(\mathbf{x} / \mathbf{y})$ $(\mathrm{DP}=\mathrm{x}+\mathrm{y}=50-500)$ in $\mathrm{PhCF}_{3}$. All the lines were generated from $\Delta G_{\mathrm{h}, \mathrm{CC}},(-0.25$ $\mathrm{kJ} / \mathrm{mol})$ and $\Delta G_{\mathrm{h}, \mathrm{CA}}(+0.065 \mathrm{~kJ} / \mathrm{mol})$ using Equations 1 and 2 . 
5 UV-vis Absorption and CD Spectra of New Compounds

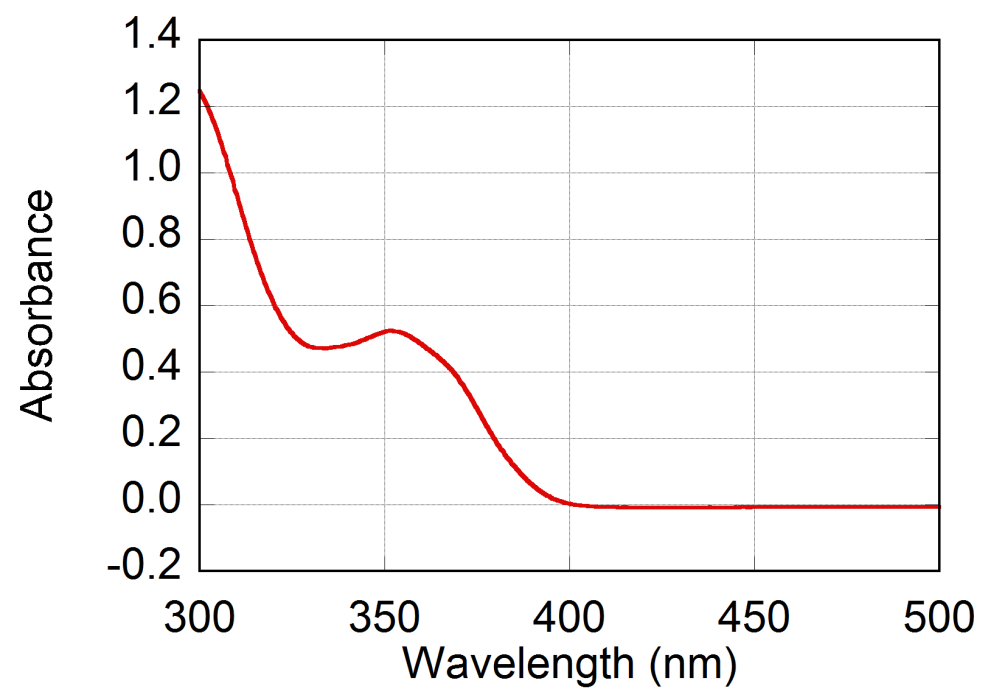

Figure S9. UV-vis absorption spectrum of $1 \mathbf{a ( 1 0 0 )}$ in $\mathrm{PhH}\left(2.01 \times 10^{-2} \mathrm{~g} / \mathrm{L}\right.$, light path length $=10 \mathrm{~mm}$ ).

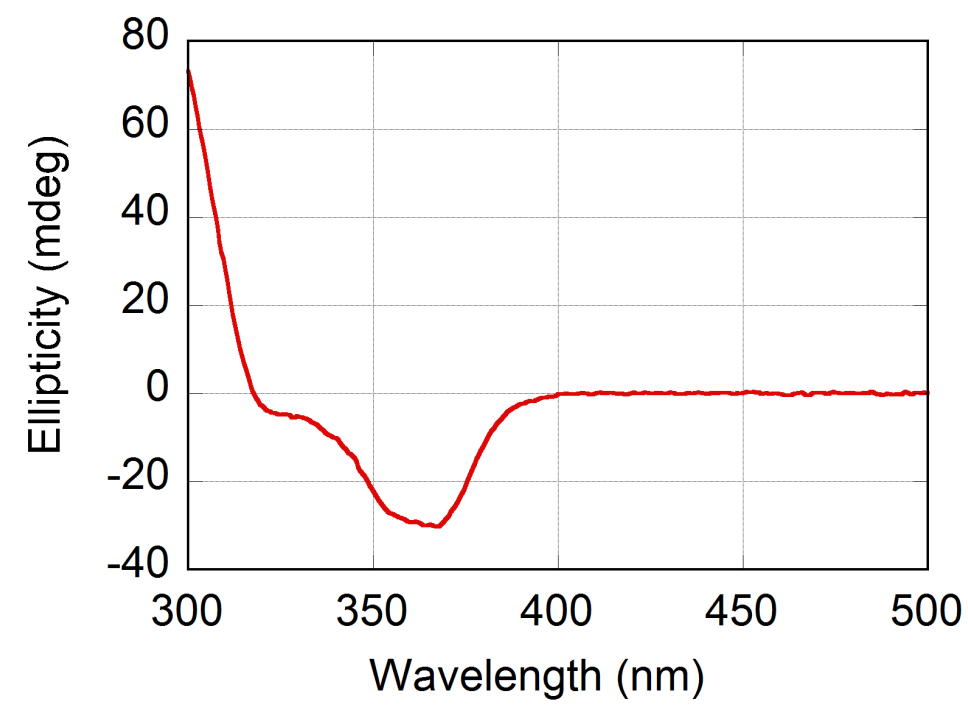

Figure S10. CD spectrum of $\mathbf{1 a ( 1 0 0 )}$ in $\mathrm{PhH}\left(2.01 \times 10^{-2} \mathrm{~g} / \mathrm{L}\right.$, light path length $=10$ $\mathrm{mm})$. 


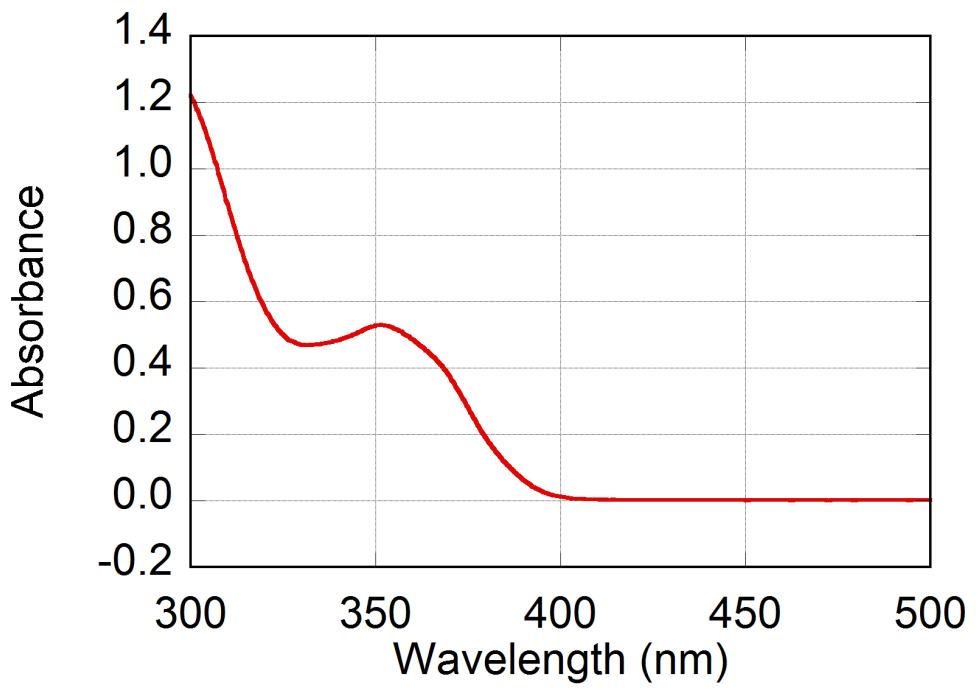

Figure S11. UV-vis absorption spectrum of $1 \mathbf{a ( 1 0 0 )}$ in $\mathrm{PhCH}_{3}\left(2.01 \times 10^{-2} \mathrm{~g} / \mathrm{L}\right.$, light path length $=10 \mathrm{~mm}$ ).

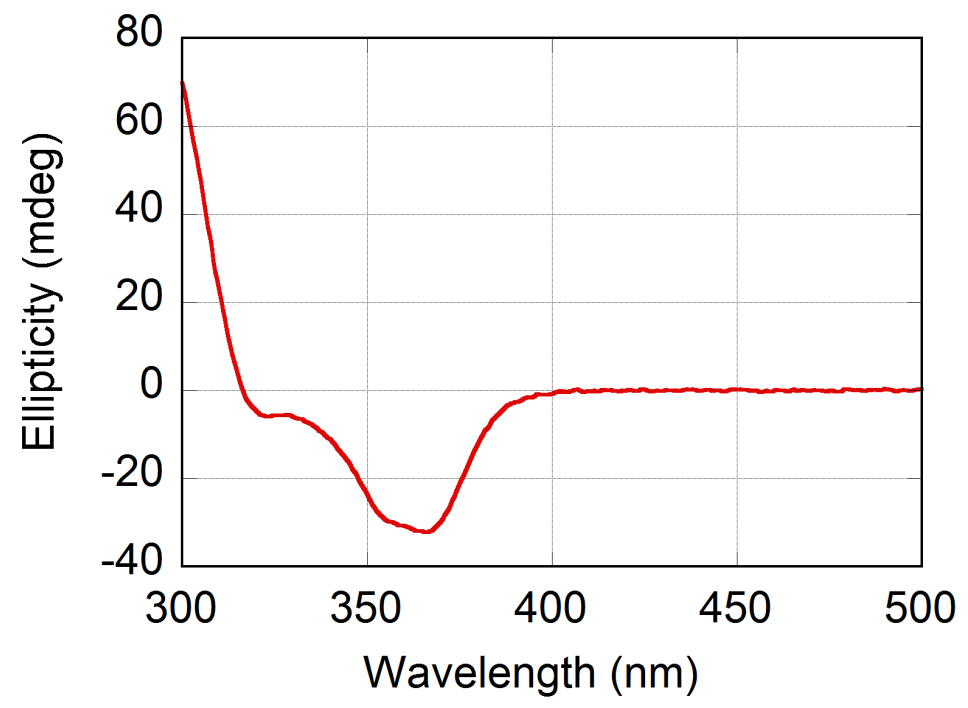

Figure S12. CD spectrum of $1 \mathbf{a ( 1 0 0 )}$ in $\mathrm{PhCH}_{3}\left(2.01 \times 10^{-2} \mathrm{~g} / \mathrm{L}\right.$, light path length $=10$ $\mathrm{mm})$. 


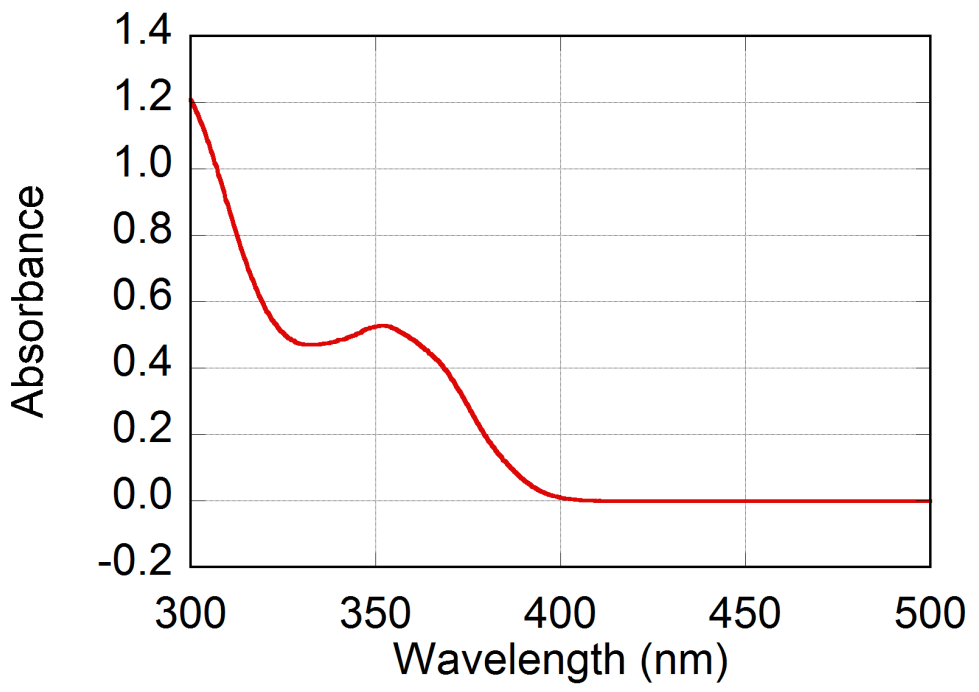

Figure S13. UV-vis absorption spectrum of 1a(100) in $\mathrm{PhOCH}_{3}\left(2.01 \times 10^{-2} \mathrm{~g} / \mathrm{L}\right.$, light path length $=10 \mathrm{~mm}$ ).

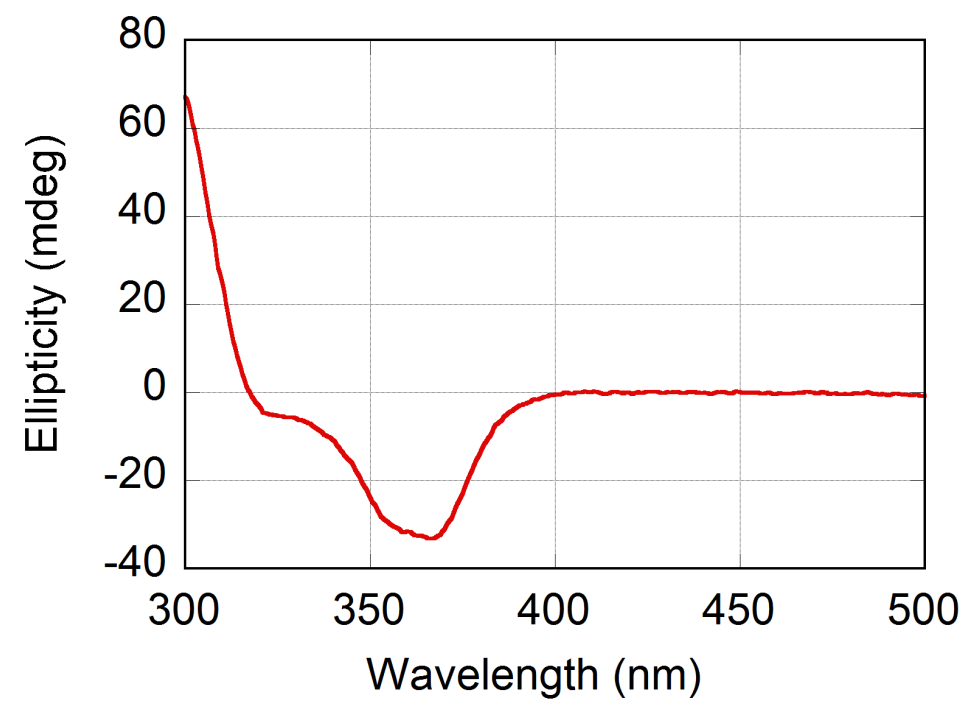

Figure S14. CD spectrum of $\mathbf{1 a}(\mathbf{1 0 0})$ in $\mathrm{PhOCH}_{3}\left(2.01 \times 10^{-2} \mathrm{~g} / \mathrm{L}\right.$, light path length $=$ $10 \mathrm{~mm}$ ). 


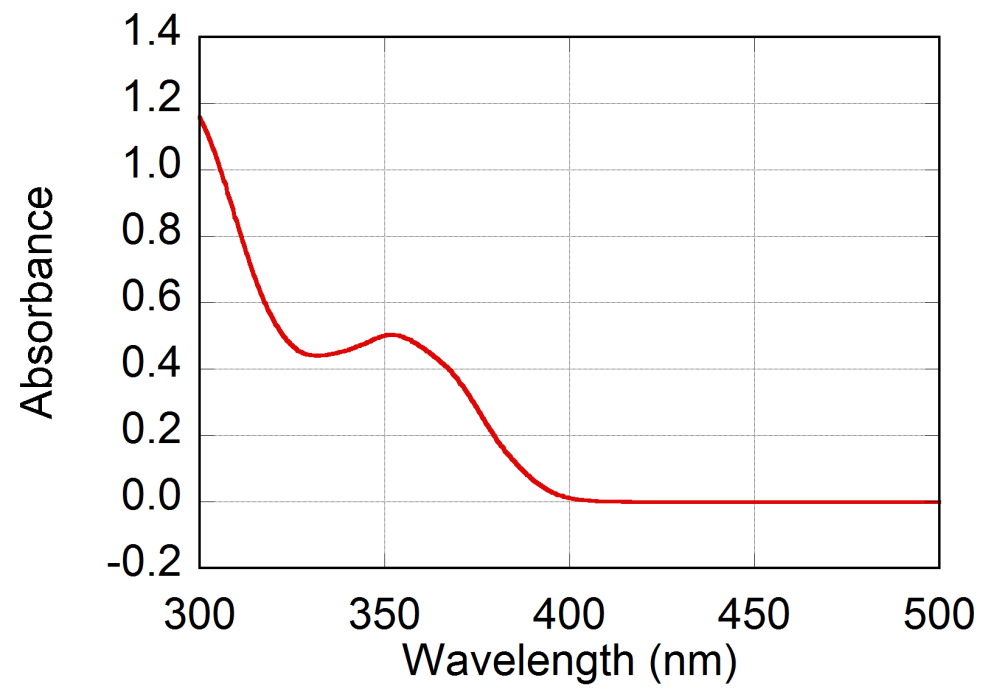

Figure S15. UV-vis absorption spectrum of 1a(100) in $\mathrm{PhCF}_{3}\left(2.01 \times 10^{-2} \mathrm{~g} / \mathrm{L}\right.$, light path length $=10 \mathrm{~mm}$ ).

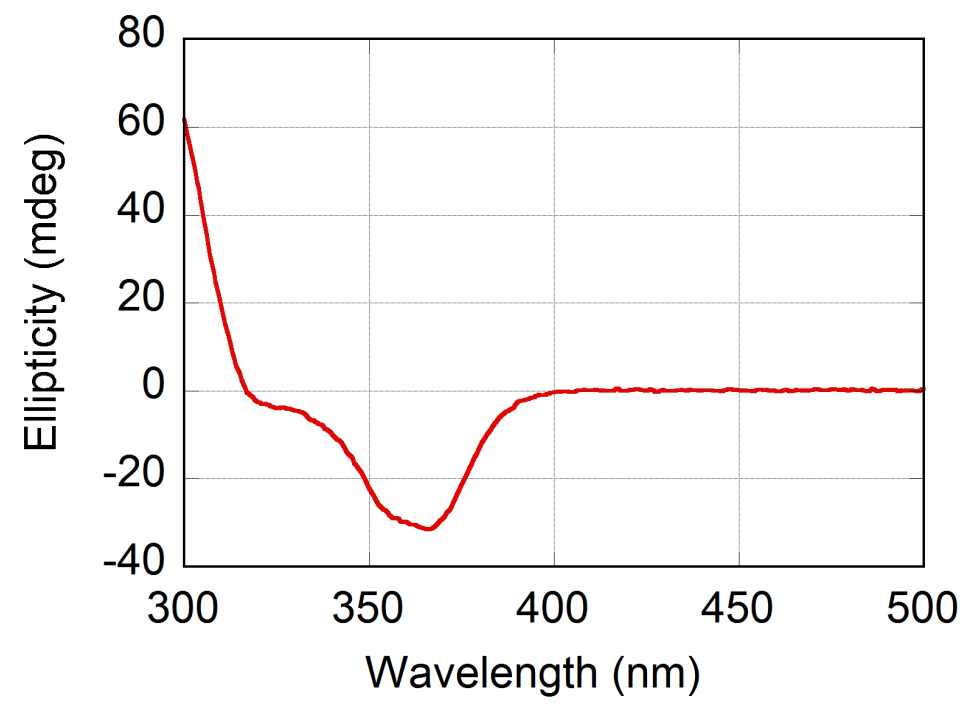

Figure S16. CD spectrum of $\mathbf{1 a ( 1 0 0 )}$ in $\mathrm{PhCF}_{3}\left(2.01 \times 10^{-2} \mathrm{~g} / \mathrm{L}\right.$, light path length $=10$ $\mathrm{mm})$. 


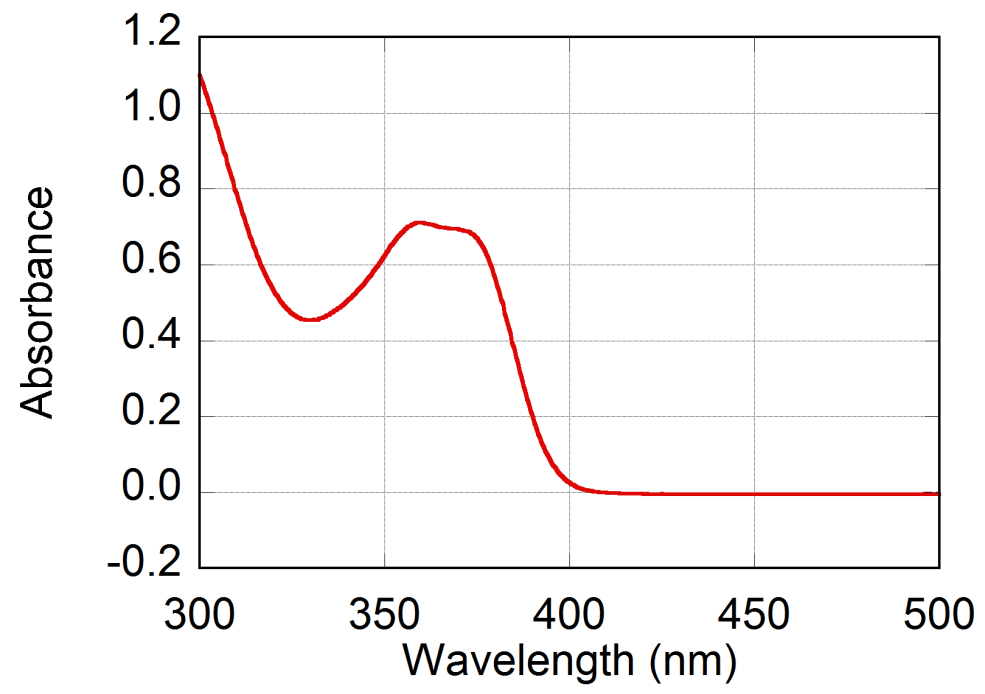

Figure S17. UV-vis absorption spectrum of $\mathbf{1 b}(\mathbf{1 0 0})$ in $\mathrm{PhH}\left(2.30 \times 10^{-2} \mathrm{~g} / \mathrm{L}\right.$, light path length $=10 \mathrm{~mm}$ ).

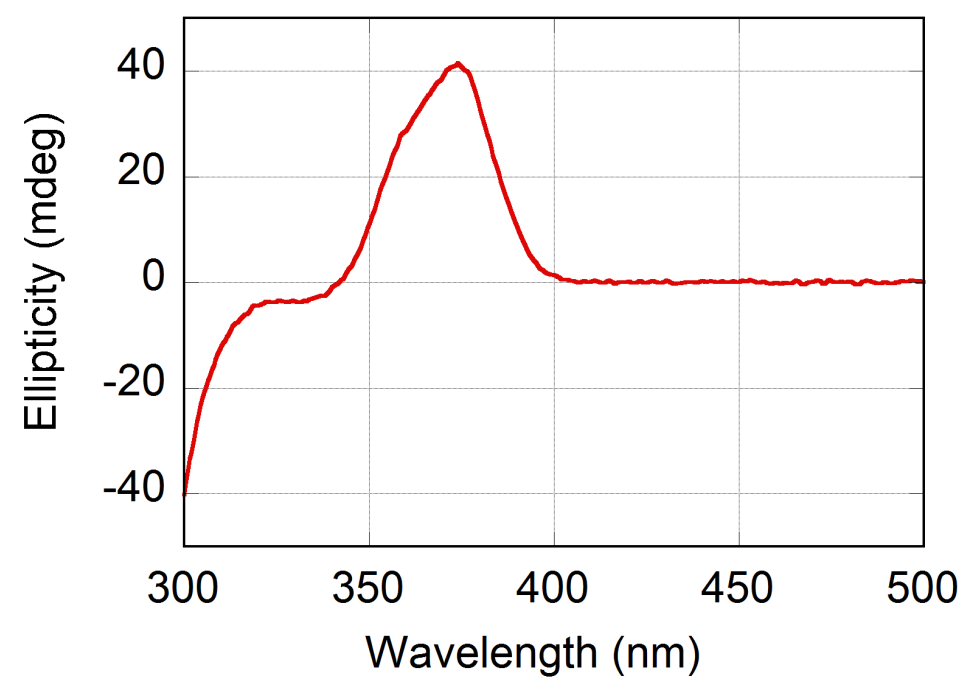

Figure S18. CD spectrum of $\mathbf{1 b}(\mathbf{1 0 0})$ in $\mathrm{PhH}\left(2.30 \times 10^{-2} \mathrm{~g} / \mathrm{L}\right.$, light path length $=10$ $\mathrm{mm}$ ). 


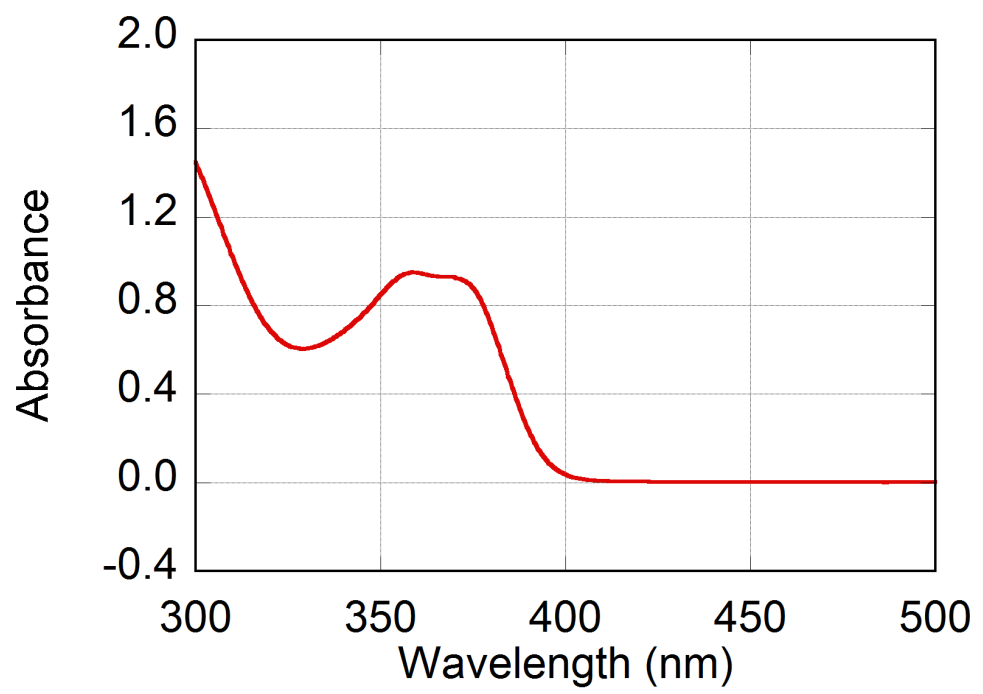

Figure S19. UV-vis absorption spectrum of $\mathbf{1 b}(\mathbf{1 0 0})$ in $\mathrm{PhCH}_{3}\left(2.30 \times 10^{-2} \mathrm{~g} / \mathrm{L}\right.$, light path length $=10 \mathrm{~mm}$ ).

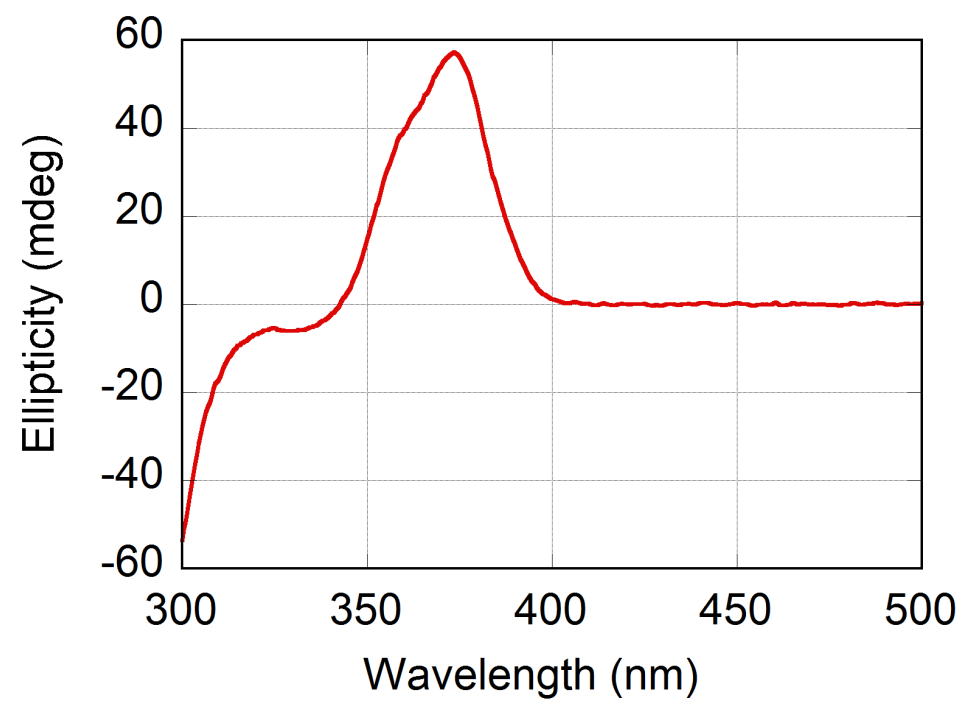

Figure S20. CD spectrum of $\mathbf{1 b}(\mathbf{1 0 0})$ in $\mathrm{PhCH}_{3}\left(2.30 \times 10^{-2} \mathrm{~g} / \mathrm{L}\right.$, light path length $=10$ $\mathrm{mm})$. 


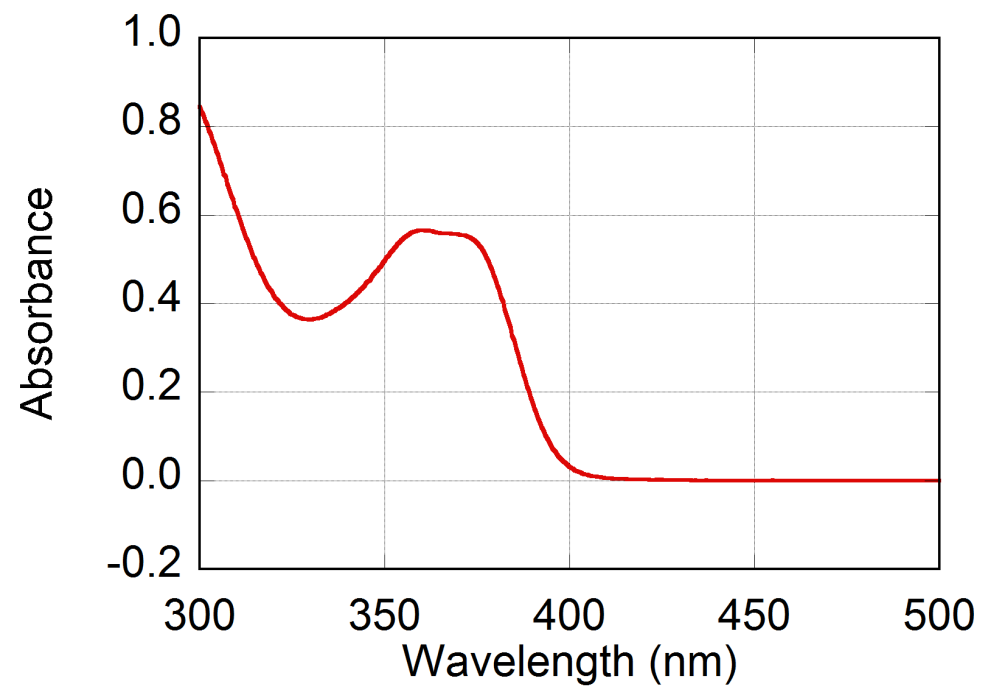

Figure S21. UV-vis absorption spectrum of 1b(100) in $\mathrm{PhOCH}_{3}\left(2.30 \times 10^{-2} \mathrm{~g} / \mathrm{L}\right.$, light path length $=10 \mathrm{~mm}$ ).

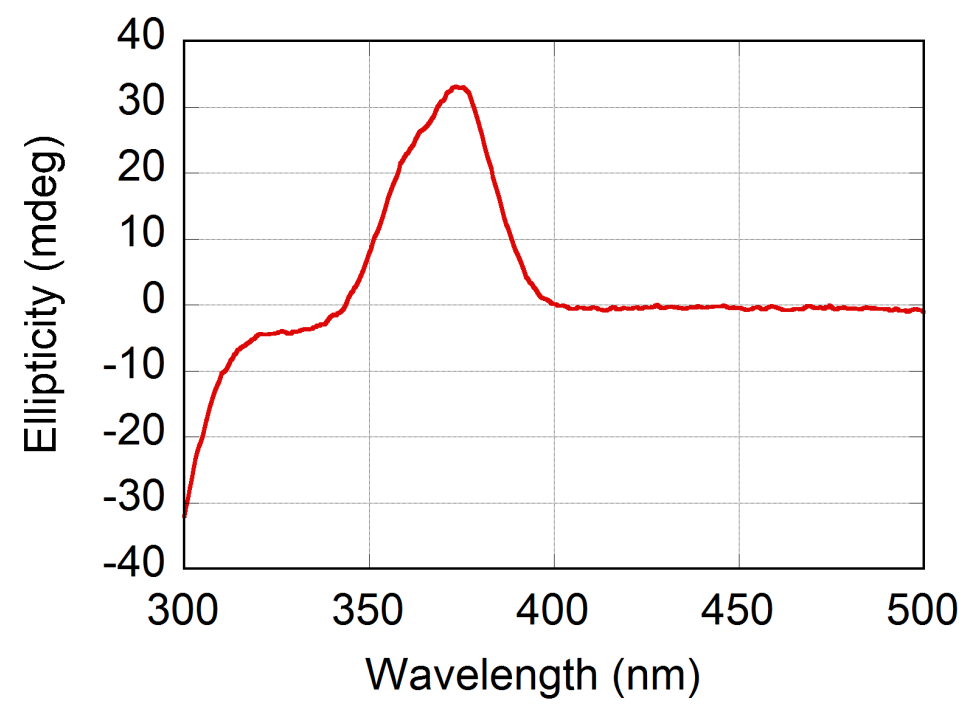

Figure S22. CD spectrum of $\mathbf{1 b}(\mathbf{1 0 0})$ in $\mathrm{PhOCH}_{3}\left(2.30 \times 10^{-2} \mathrm{~g} / \mathrm{L}\right.$, light path length $=$ $10 \mathrm{~mm}$ ). 


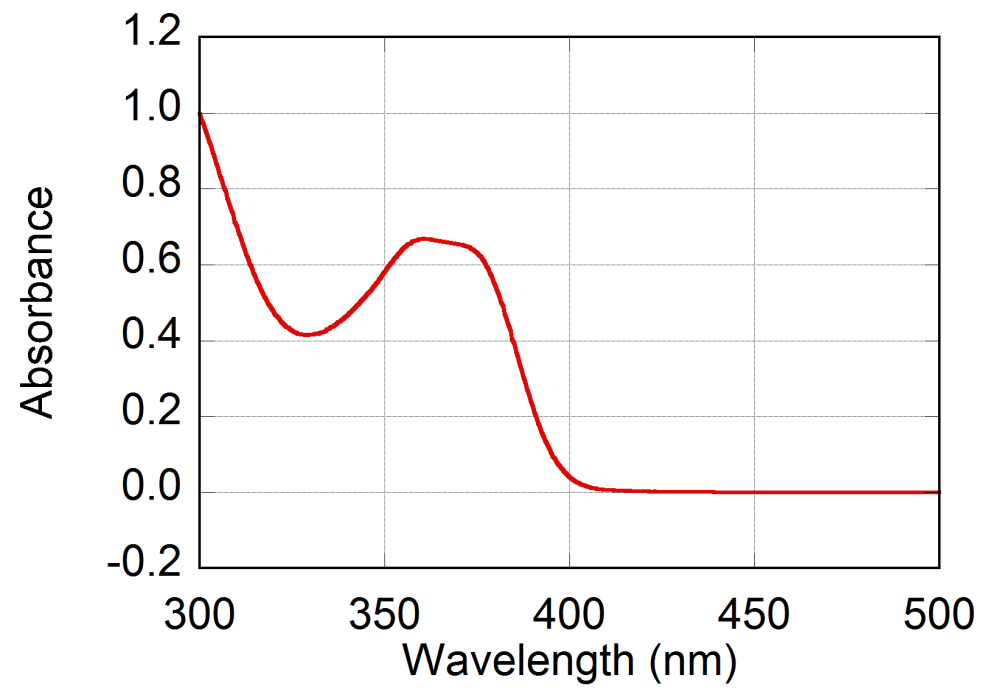

Figure S23. UV-vis absorption spectrum of $\mathbf{1 b}(\mathbf{1 0 0})$ in $\mathrm{PhCF}_{3}\left(2.30 \times 10^{-2} \mathrm{~g} / \mathrm{L}\right.$, light path length $=10 \mathrm{~mm}$ ).

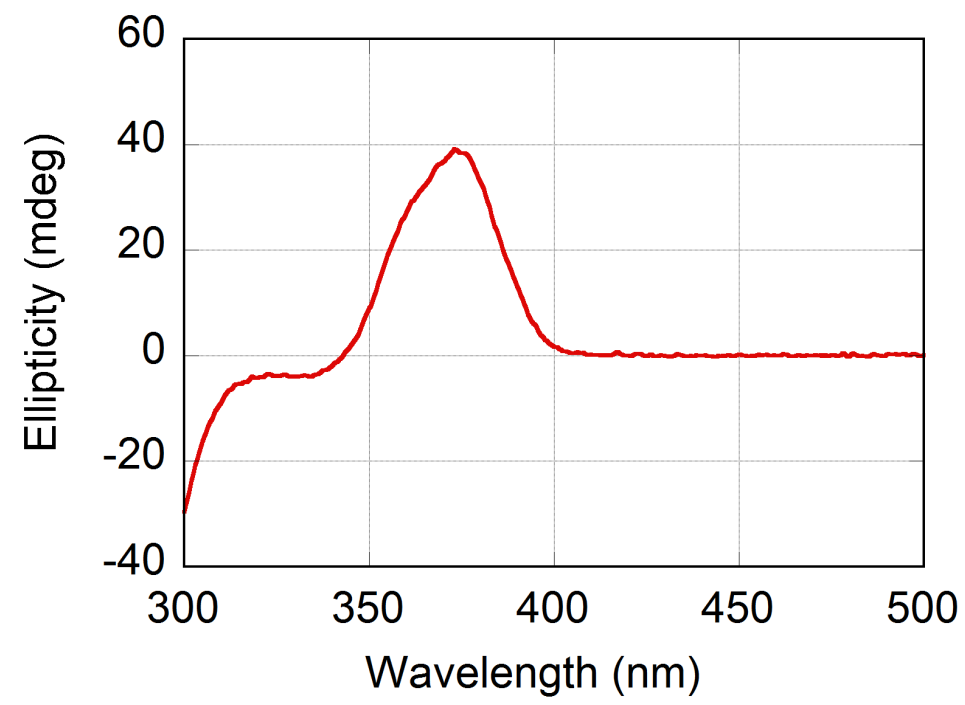

Figure S24. UV-vis absorption spectrum of $\mathbf{1 b}(\mathbf{1 0 0})$ in $\mathrm{PhCF}_{3}\left(2.30 \times 10^{-2} \mathrm{~g} / \mathrm{L}\right.$, light path length $=10 \mathrm{~mm}$ ). 


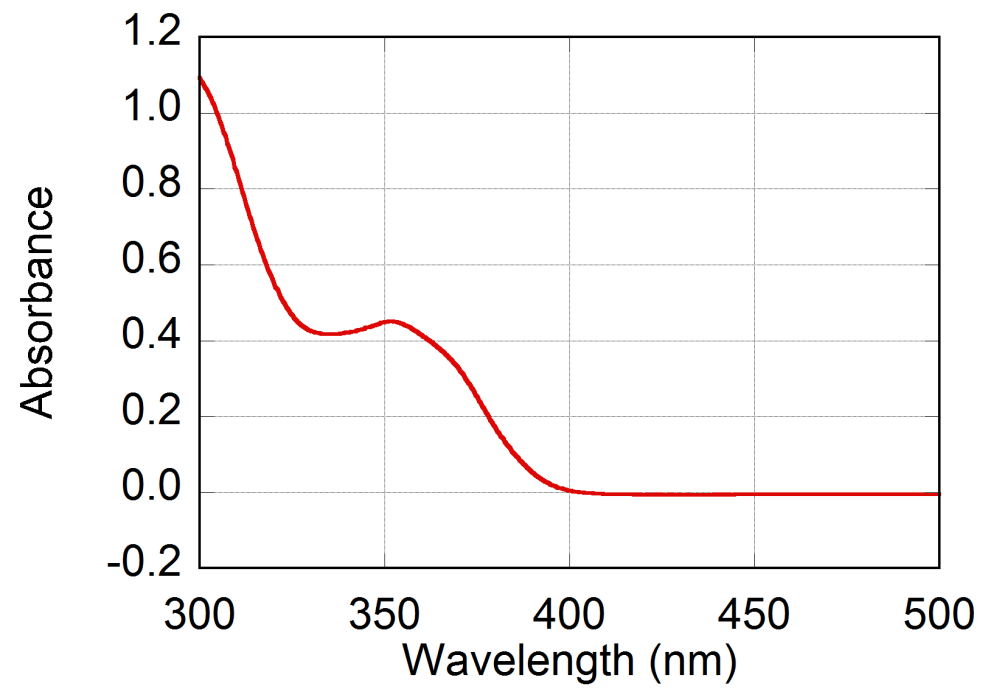

Figure S25. UV-vis absorption spectrum of 1c(100) in $\mathrm{PhH}\left(2.07 \times 10^{-2} \mathrm{~g} / \mathrm{L}\right.$, light path length $=10 \mathrm{~mm}$ ).

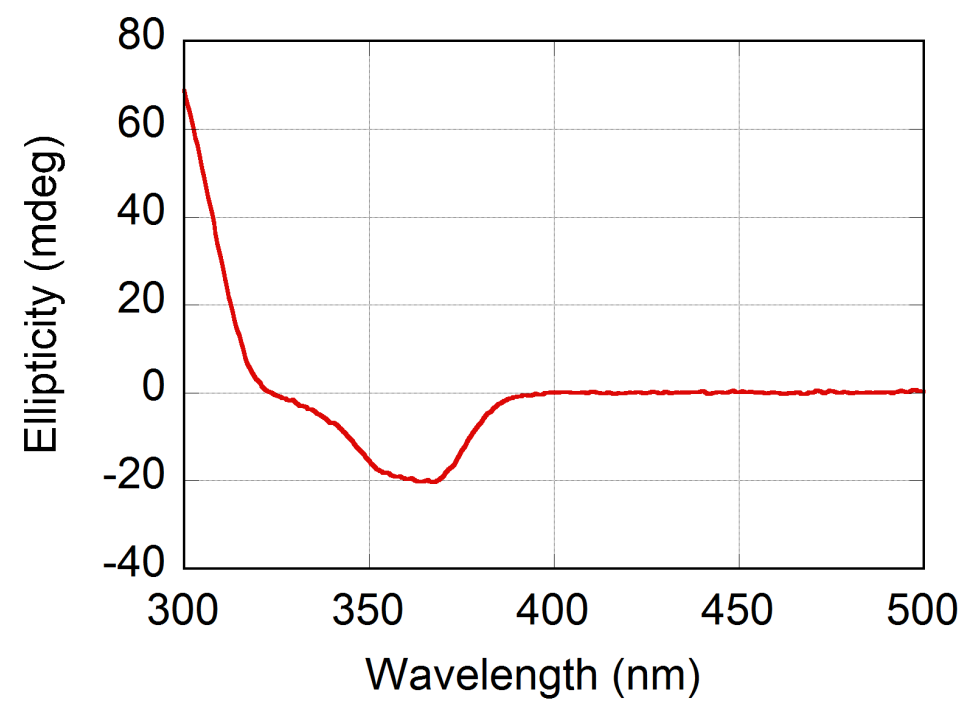

Figure S26. CD spectrum of 1c(100) in $\mathrm{PhH}\left(2.07 \times 10^{-2} \mathrm{~g} / \mathrm{L}\right.$, light path length $=10$ $\mathrm{mm})$. 


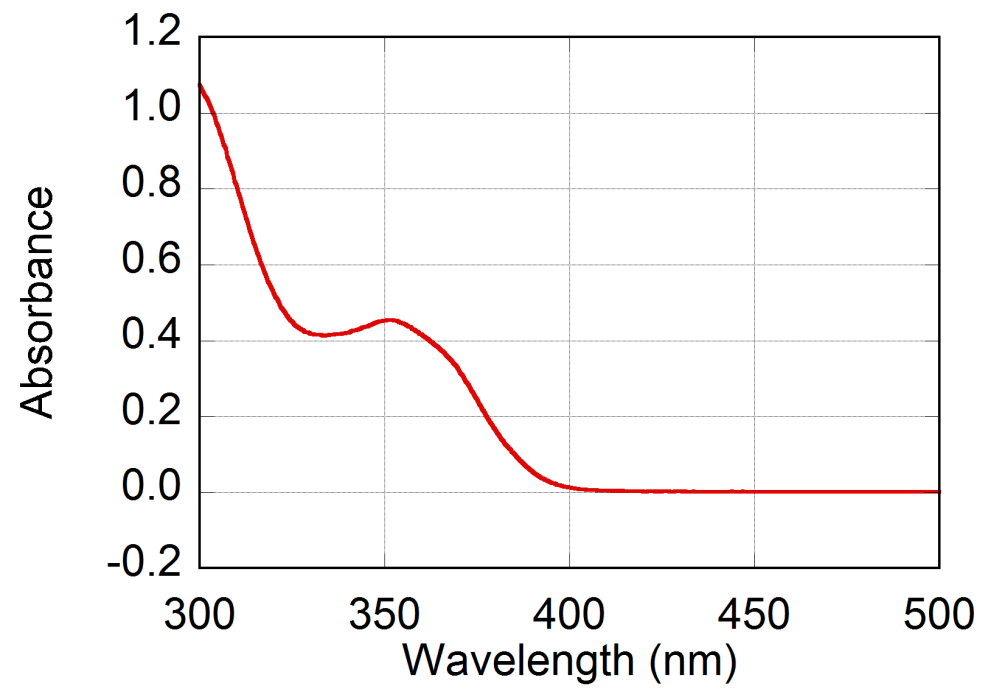

Figure S27. UV-vis absorption spectrum of 1c(100) in $\mathrm{PhCH}_{3}\left(2.07 \times 10^{-2} \mathrm{~g} / \mathrm{L}\right.$, light path length $=10 \mathrm{~mm}$ ).

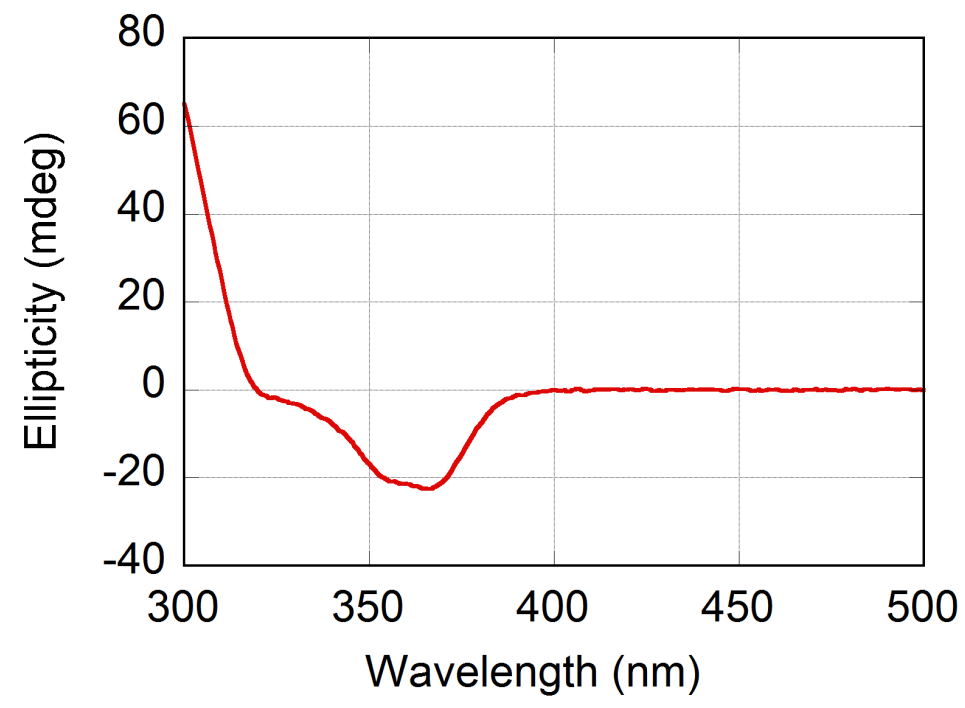

Figure S28. CD spectrum of $\mathbf{1 c ( 1 0 0 )}$ in $\mathrm{PhCH}_{3}\left(2.07 \times 10^{-2} \mathrm{~g} / \mathrm{L}\right.$, light path length $=10$ $\mathrm{mm}$ ). 


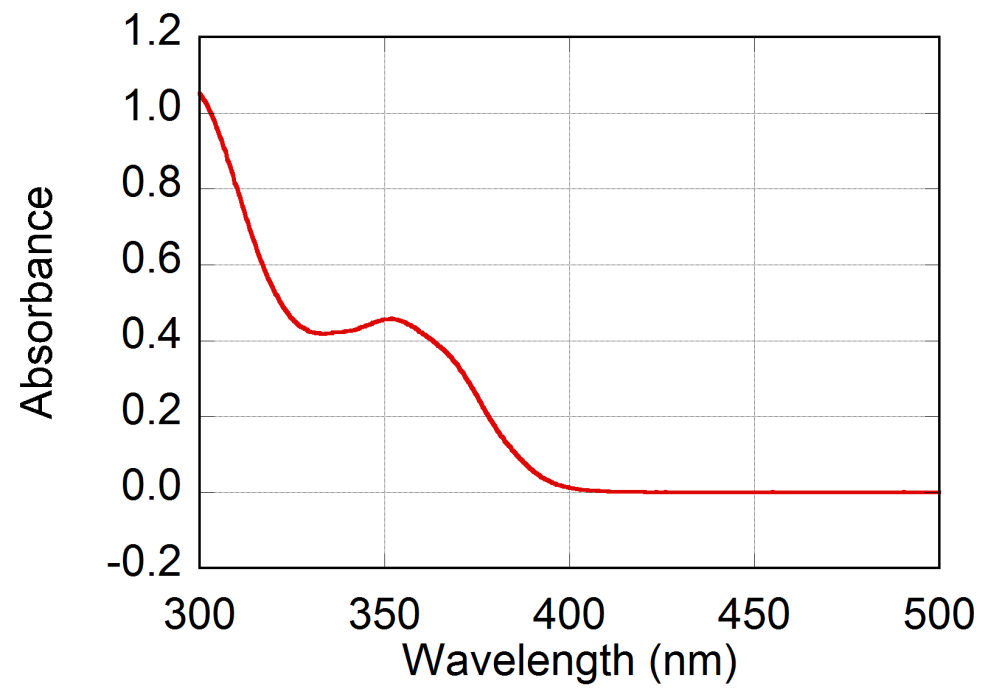

Figure S29. UV-vis absorption spectrum of 1c(100) in $\mathrm{PhOCH}_{3}\left(2.07 \times 10^{-2} \mathrm{~g} / \mathrm{L}\right.$, light path length $=10 \mathrm{~mm}$ ).

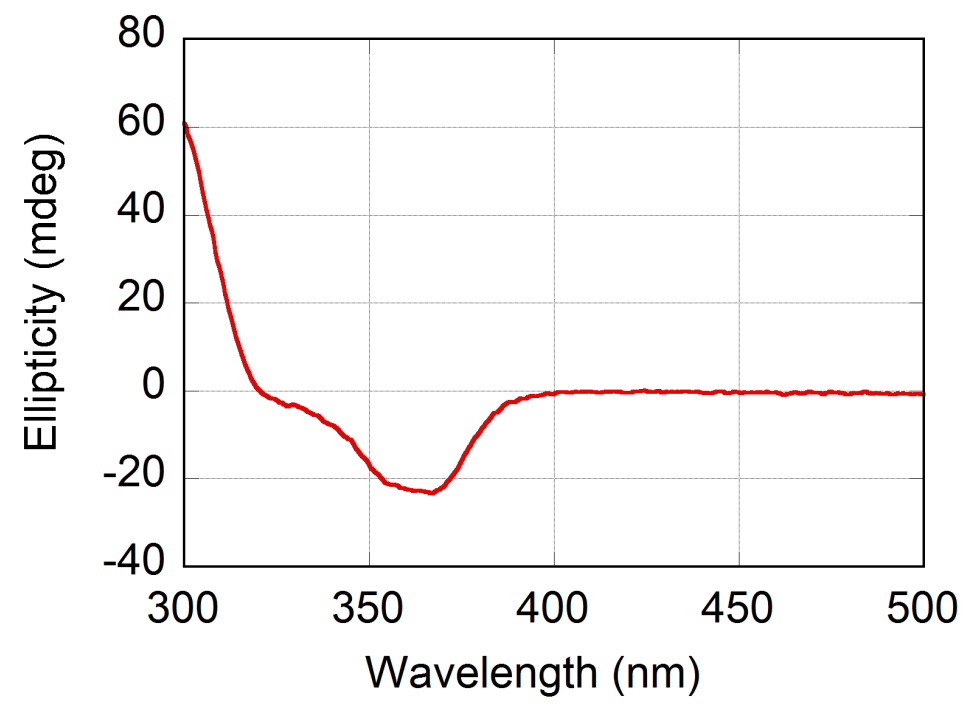

Figure S30. CD spectrum of $\mathbf{1 c ( 1 0 0 )}$ in $\mathrm{PhOCH}_{3}\left(2.07 \times 10^{-2} \mathrm{~g} / \mathrm{L}\right.$, light path length $=10$ $\mathrm{mm})$. 


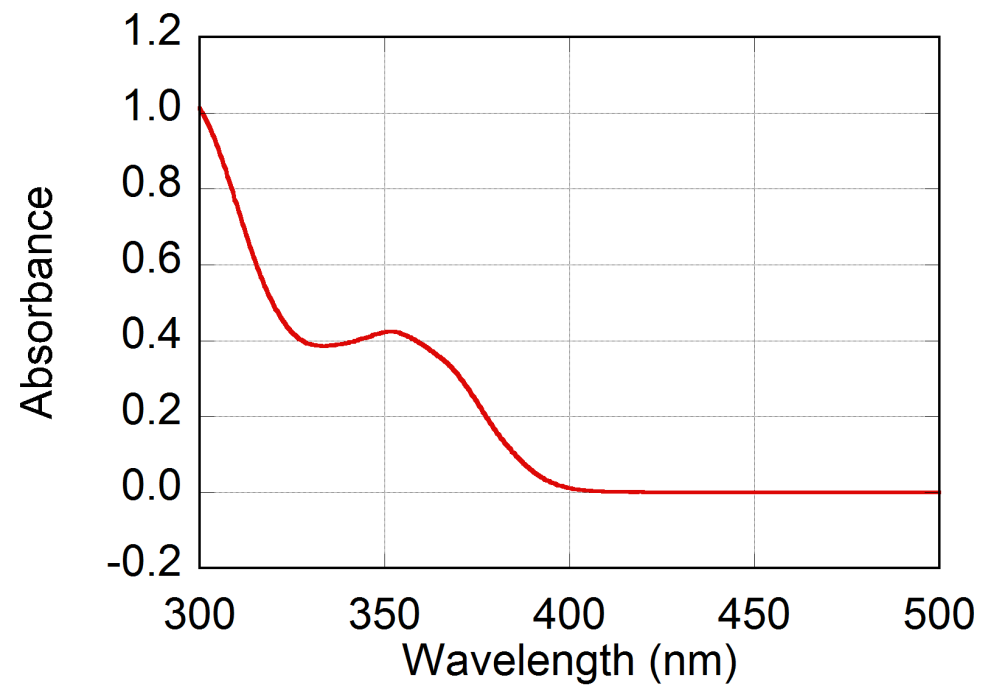

Figure S31. UV-vis absorption spectrum of 1c(100) in $\mathrm{PhCF}_{3}\left(2.07 \times 10^{-2} \mathrm{~g} / \mathrm{L}\right.$, light path length $=10 \mathrm{~mm}$ ).

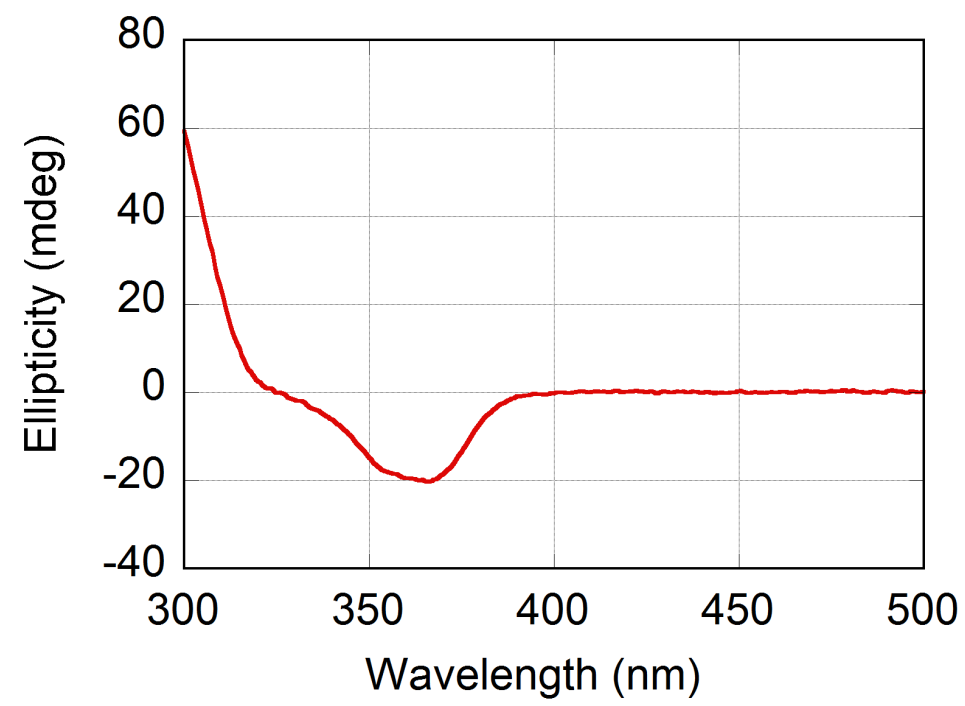

Figure S32. CD spectrum of 1c(100) in $\mathrm{PhCF}_{3}\left(2.07 \times 10^{-2} \mathrm{~g} / \mathrm{L}\right.$, light path length $=10$ $\mathrm{mm}$ ). 


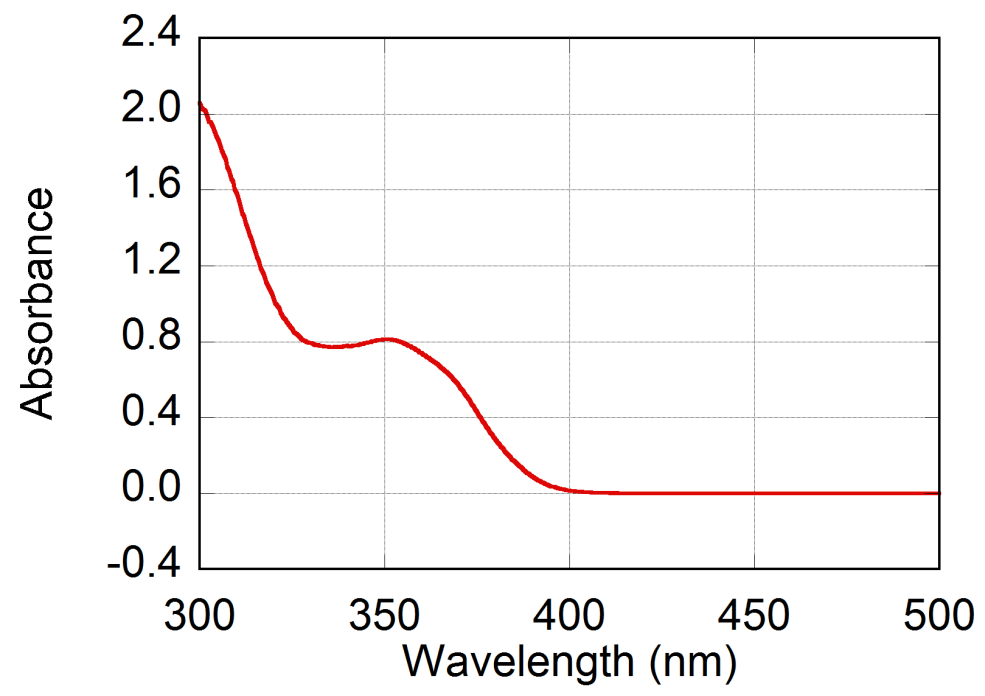

Figure S33. UV-vis absorption spectrum of $\mathbf{1 d ( 1 0 0 )}$ in $\mathrm{PhH}\left(2.44 \times 10^{-2} \mathrm{~g} / \mathrm{L}\right.$, light path length $=10 \mathrm{~mm}$ ).

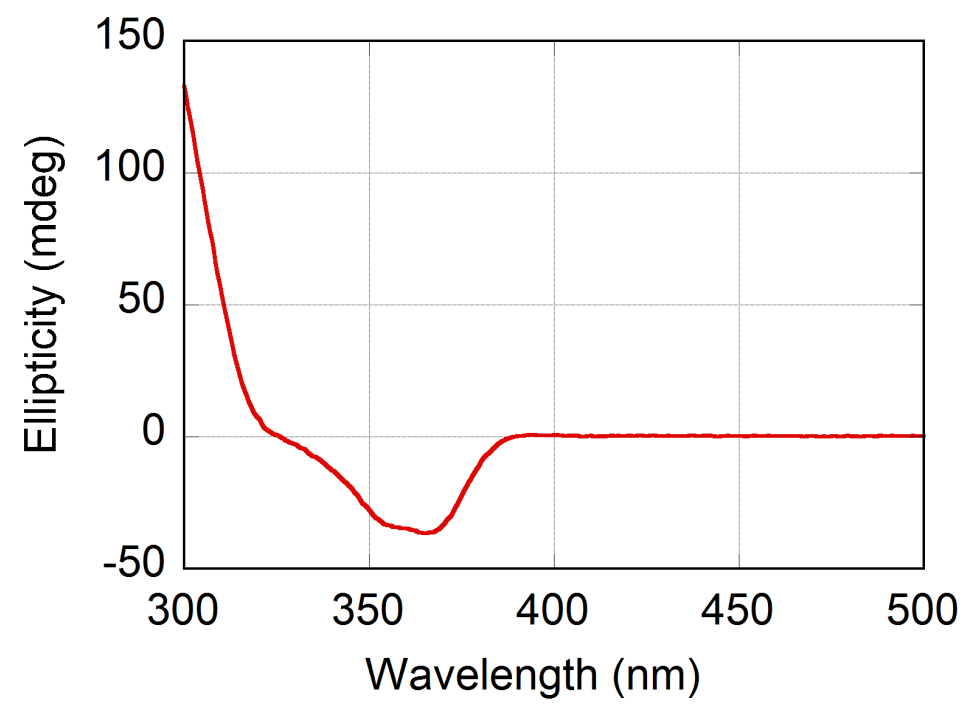

Figure S34. CD spectrum of $\mathbf{1 d ( 1 0 0 )}$ in $\mathrm{PhH}\left(2.44 \times 10^{-2} \mathrm{~g} / \mathrm{L}\right.$, light path length $=10$ $\mathrm{mm})$. 


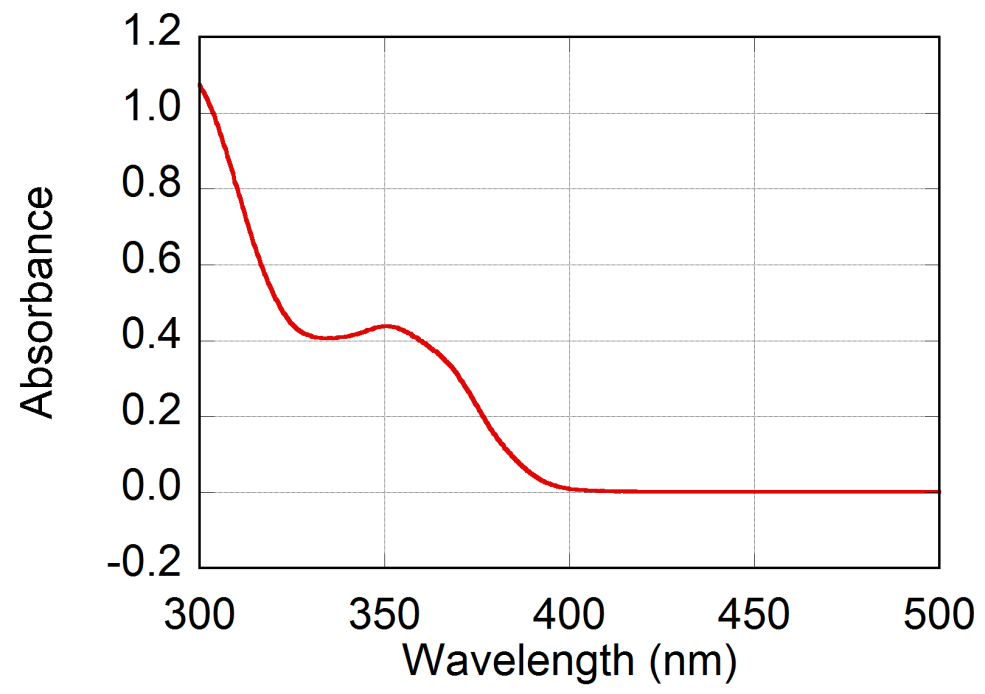

Figure S35. UV-vis absorption spectrum of 1d(100) in $\mathrm{PhCH}_{3}\left(2.44 \times 10^{-2} \mathrm{~g} / \mathrm{L}\right.$, light path length $=10 \mathrm{~mm}$ ).

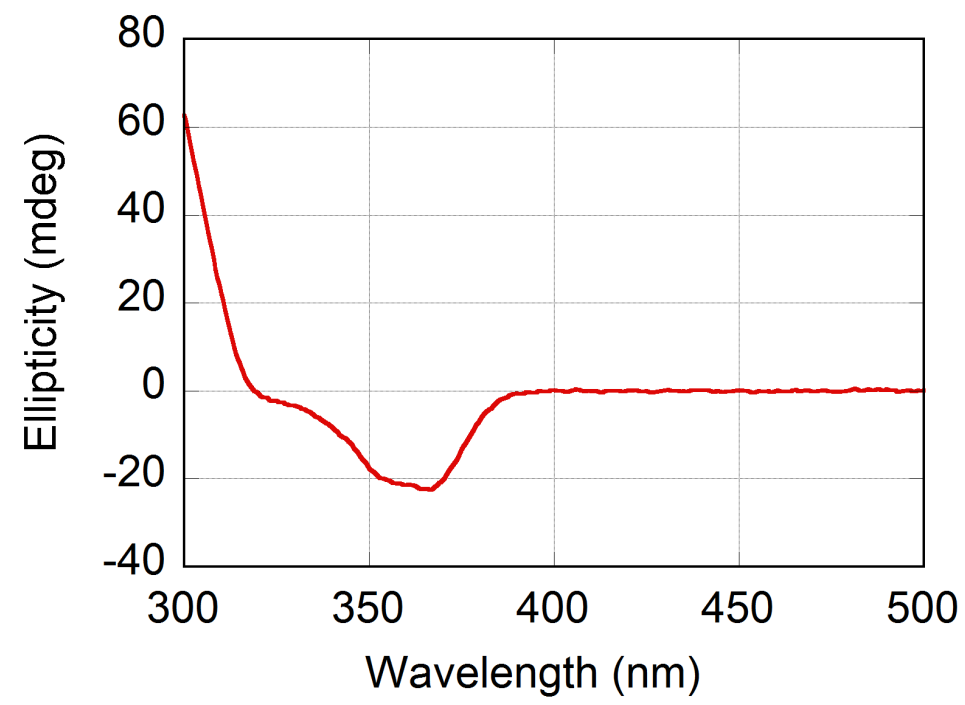

Figure S36. CD spectrum of $\mathbf{1 d ( 1 0 0 )}$ in $\mathrm{PhCH}_{3}\left(2.44 \times 10^{-2} \mathrm{~g} / \mathrm{L}\right.$, light path length $=10$ $\mathrm{mm})$. 


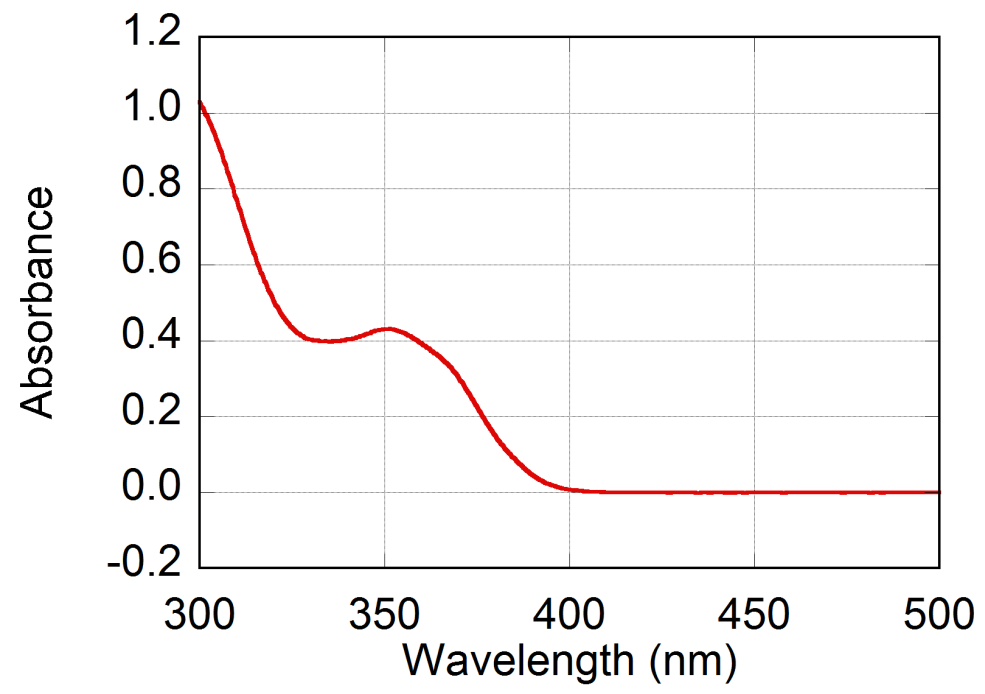

Figure S37. UV-vis absorption spectrum of 1d(100) in $\mathrm{PhOCH}_{3}\left(2.44 \times 10^{-2} \mathrm{~g} / \mathrm{L}\right.$, light path length $=10 \mathrm{~mm}$ ).

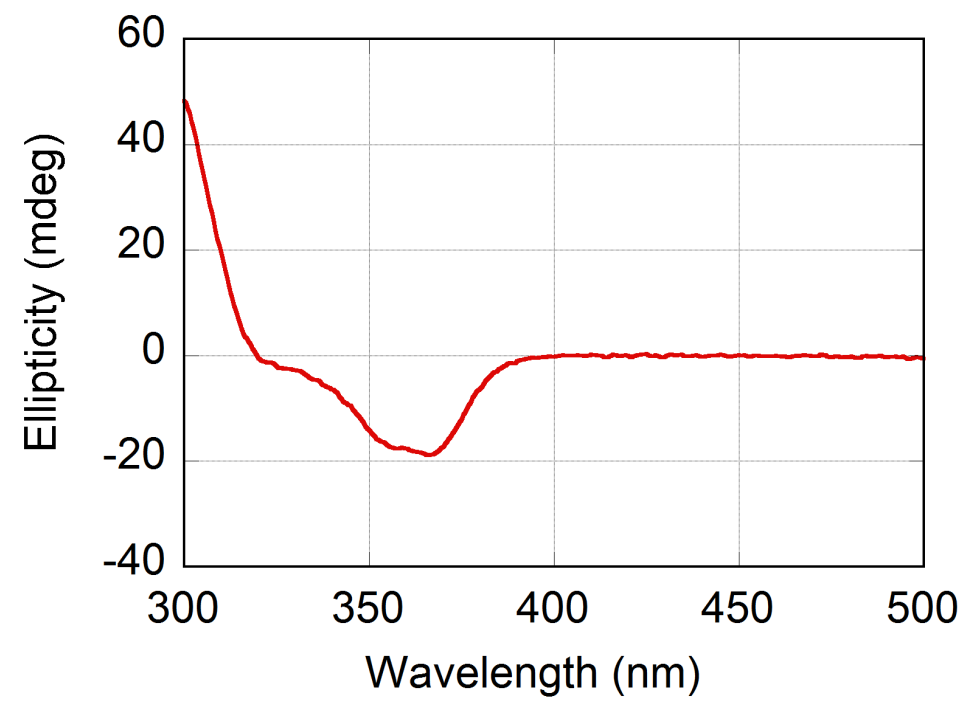

Figure S38. CD spectrum of $\mathbf{1 d}(\mathbf{1 0 0})$ in $\mathrm{PhOCH}_{3}\left(2.44 \times 10^{-2} \mathrm{~g} / \mathrm{L}\right.$, light path length $=$ $10 \mathrm{~mm}$ ). 


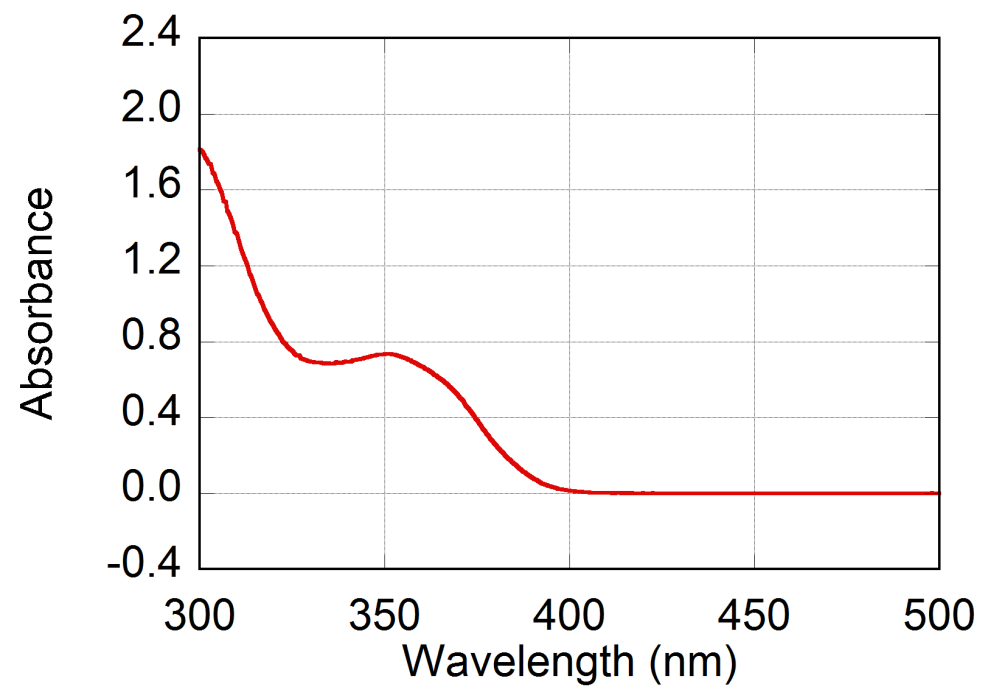

Figure S39. UV-vis absorption spectrum of $\mathbf{1 d ( 1 0 0 )}$ in $\mathrm{PhCF}_{3}\left(2.44 \times 10^{-2} \mathrm{~g} / \mathrm{L}\right.$, light path length $=10 \mathrm{~mm}$ ).

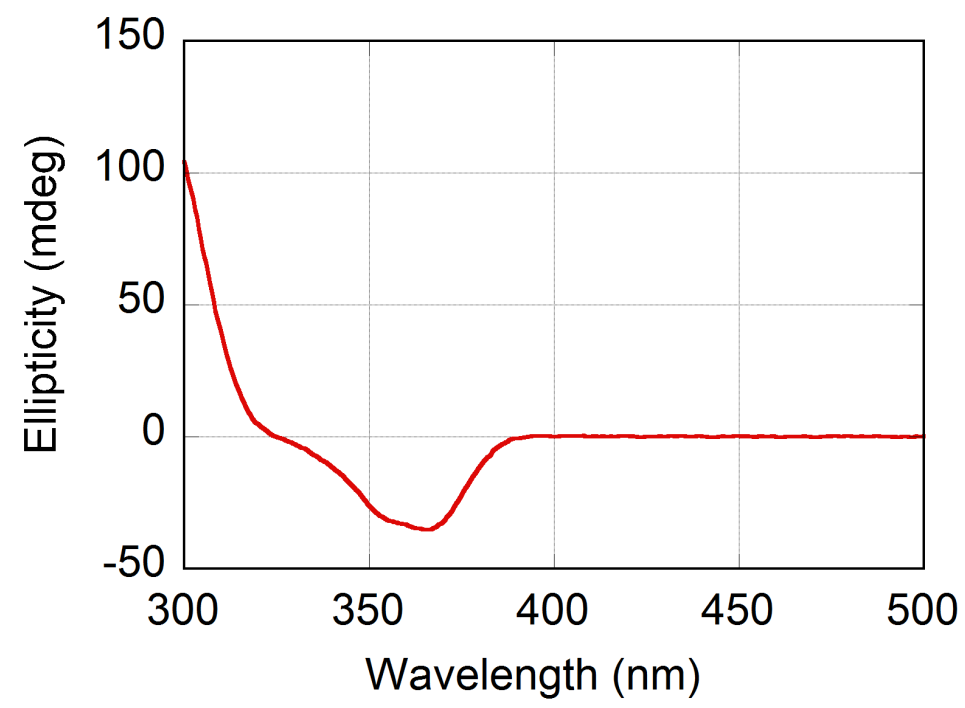

Figure S40. CD spectrum of $\mathbf{1 d ( 1 0 0 )}$ in $\mathrm{PhCF}_{3}\left(2.44 \times 10^{-2} \mathrm{~g} / \mathrm{L}\right.$, light path length $=10$ $\mathrm{mm}$ ). 


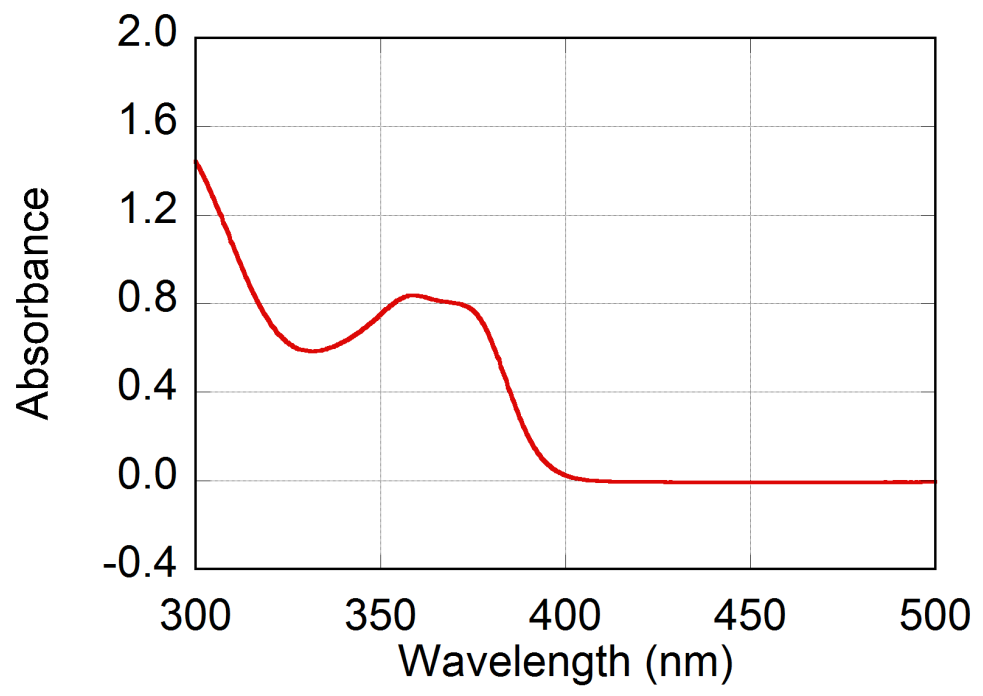

Figure S41. UV-vis absorption spectrum of $\mathbf{1 a ( 5 / 1 0 0 )}$ in $\mathrm{PhH}\left(2.62 \times 10^{-2} \mathrm{~g} / \mathrm{L}\right.$, light path length $=10 \mathrm{~mm}$ ).

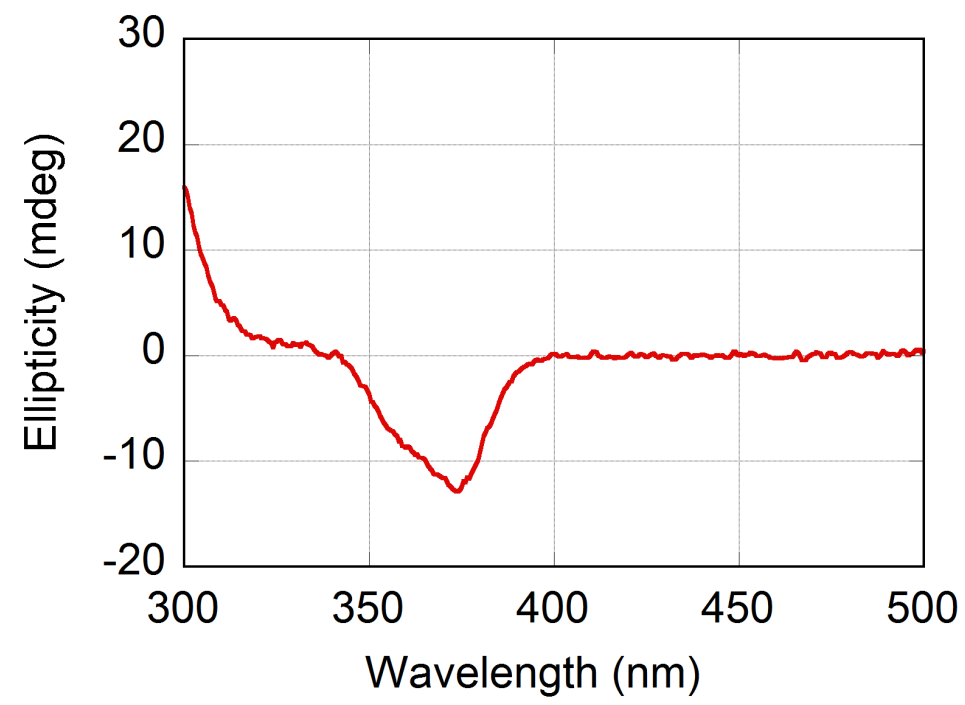

Figure S42. CD spectrum of $\mathbf{1 a ( 5 / 1 0 0})$ in $\mathrm{PhH}\left(2.62 \times 10^{-2} \mathrm{~g} / \mathrm{L}\right.$, light path length $=10$ $\mathrm{mm})$. 


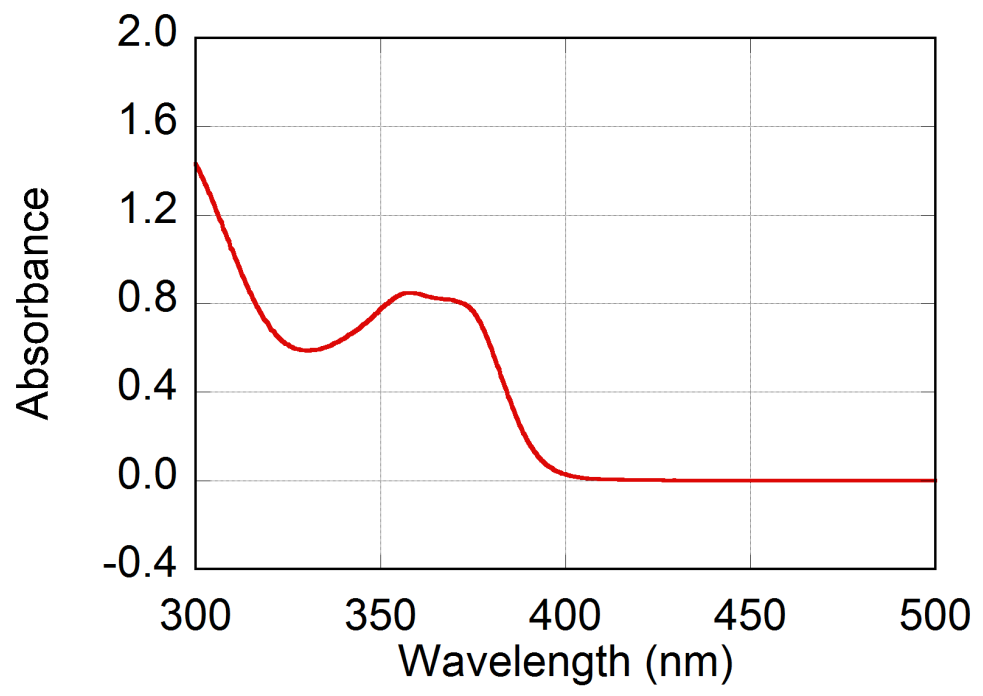

Figure S43. UV-vis absorption spectrum of $\mathbf{1 a ( 5 / 1 0 0 )}$ in $\mathrm{PhCH}_{3}\left(2.62 \times 10^{-2} \mathrm{~g} / \mathrm{L}\right.$, light path length $=10 \mathrm{~mm}$ ).

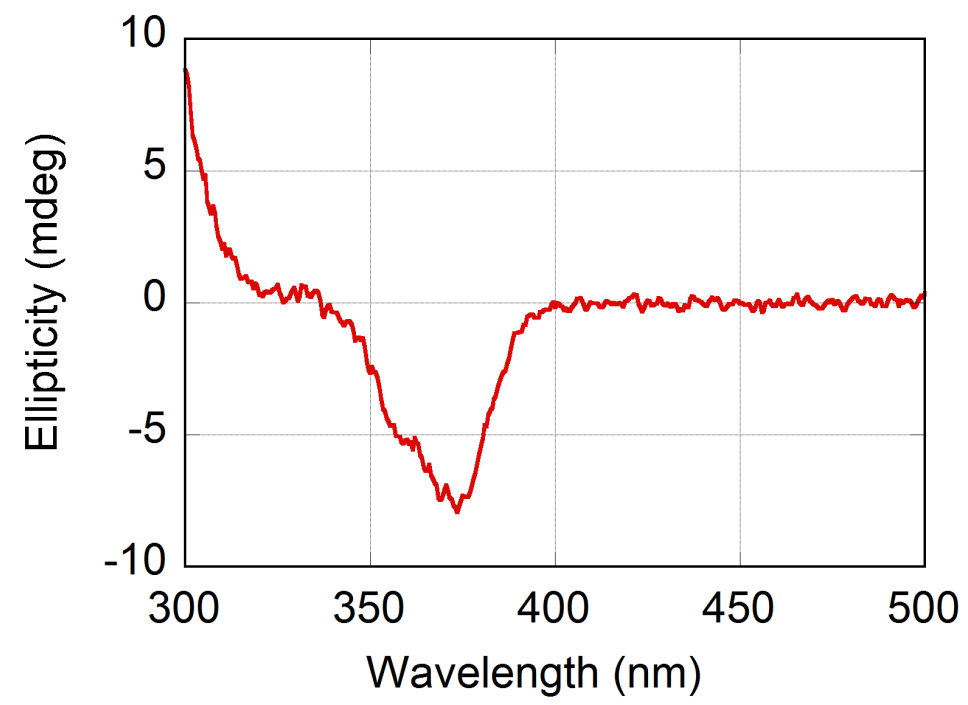

Figure S44. CD spectrum of $\mathbf{1 a ( 5 / 1 0 0})$ in $\mathrm{PhCH}_{3}\left(2.62 \times 10^{-2} \mathrm{~g} / \mathrm{L}\right.$, light path length $=$ $10 \mathrm{~mm}$ ). 


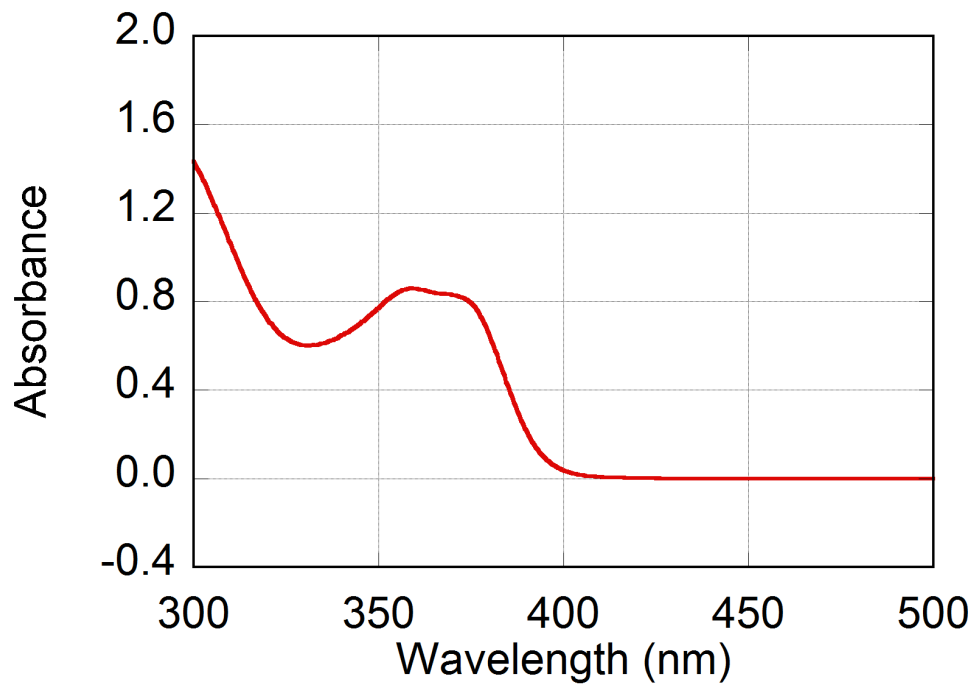

Figure S45. UV-vis absorption spectrum of $\mathbf{1 a ( 5 / 1 0 0 )}$ in $\mathrm{PhOCH}_{3}\left(2.62 \times 10^{-2} \mathrm{~g} / \mathrm{L}\right.$, light path length $=10 \mathrm{~mm}$ ).

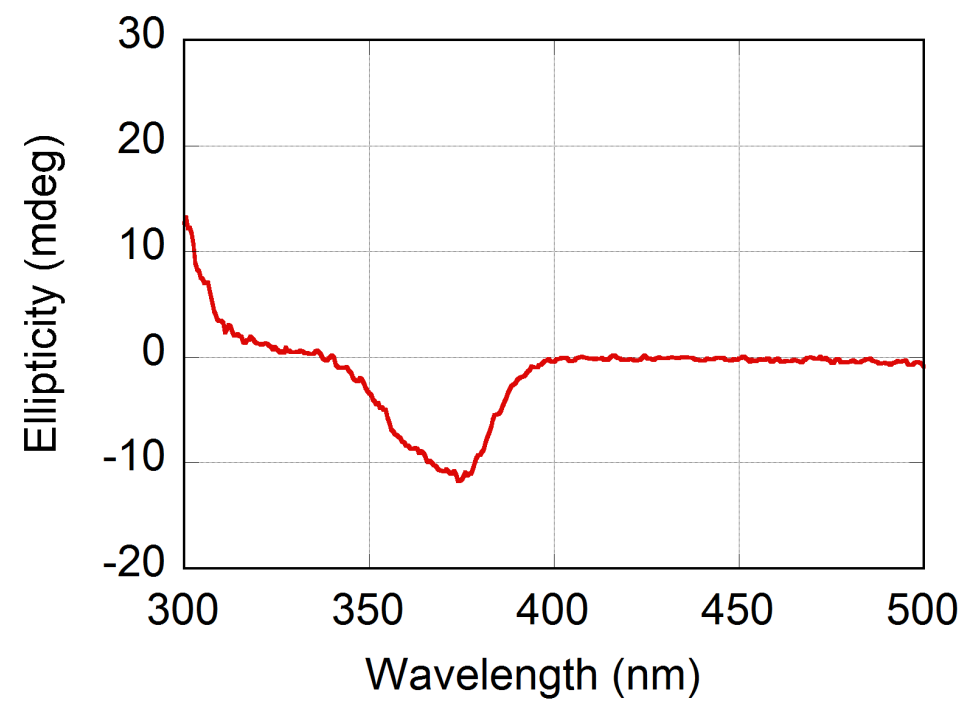

Figure S46. CD spectrum of 1a(5/100) in $\mathrm{PhOCH}_{3}\left(2.62 \times 10^{-2} \mathrm{~g} / \mathrm{L}\right.$, light path length $=$ $10 \mathrm{~mm}$ ). 


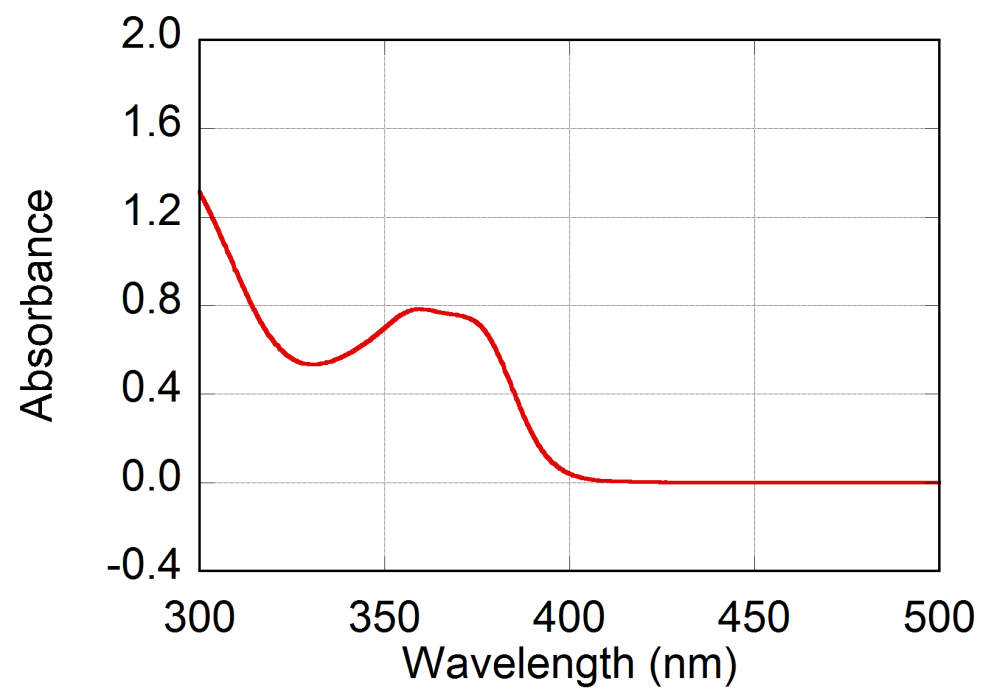

Figure S47. UV-vis absorption spectrum of $\mathbf{2 a ( 5 / 1 0 0 )}$ in $\mathrm{PhCF}_{3}\left(2.62 \times 10^{-2} \mathrm{~g} / \mathrm{L}\right.$, light path length $=10 \mathrm{~mm}$ ).

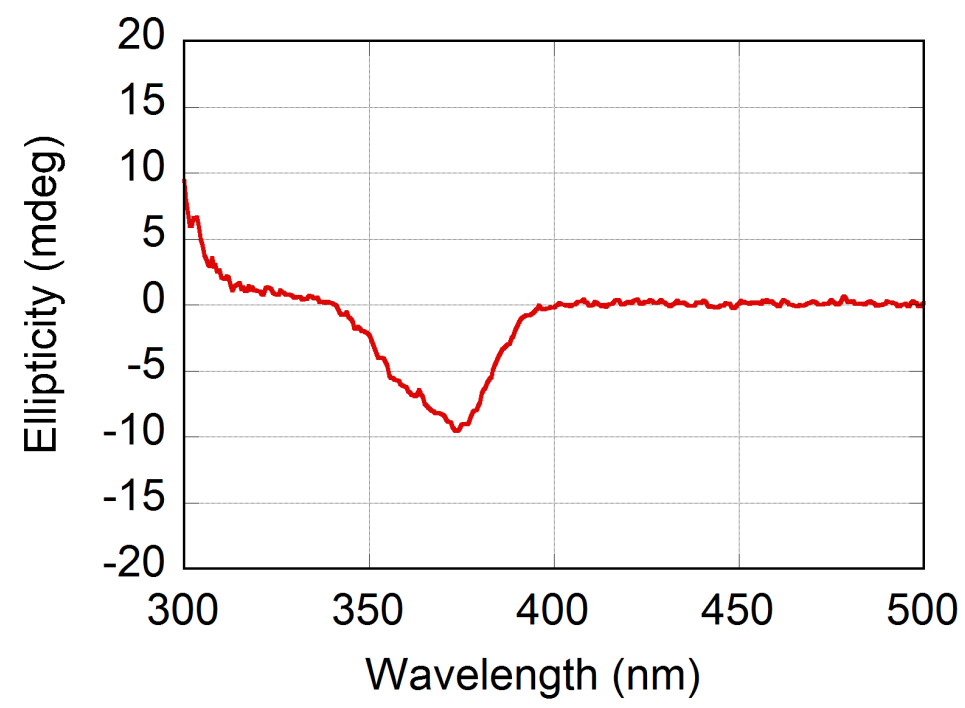

Figure S48. CD spectrum of $\mathbf{2 a ( 5 / 1 0 0 )}$ in $\mathrm{PhCF}_{3}\left(2.62 \times 10^{-2} \mathrm{~g} / \mathrm{L}\right.$, light path length $=10$ $\mathrm{mm})$. 


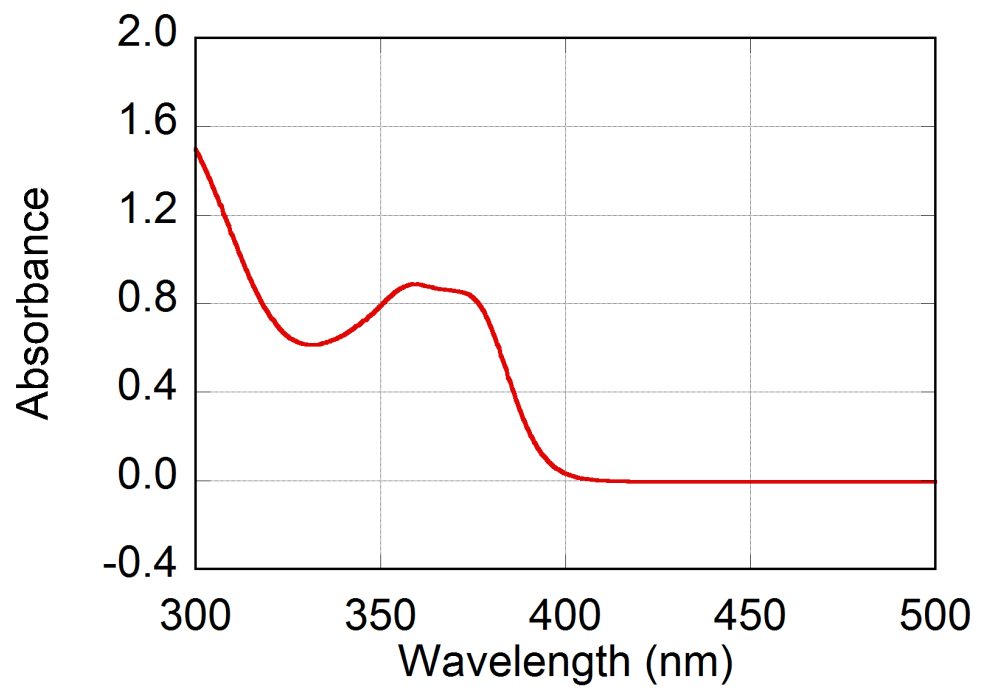

Figure S49. UV-vis absorption spectrum of $\mathbf{2 b}(\mathbf{5} / \mathbf{1 0 0})$ in $\mathrm{PhH}\left(2.48 \times 10^{-2} \mathrm{~g} / \mathrm{L}\right.$, light path length $=10 \mathrm{~mm}$ ).

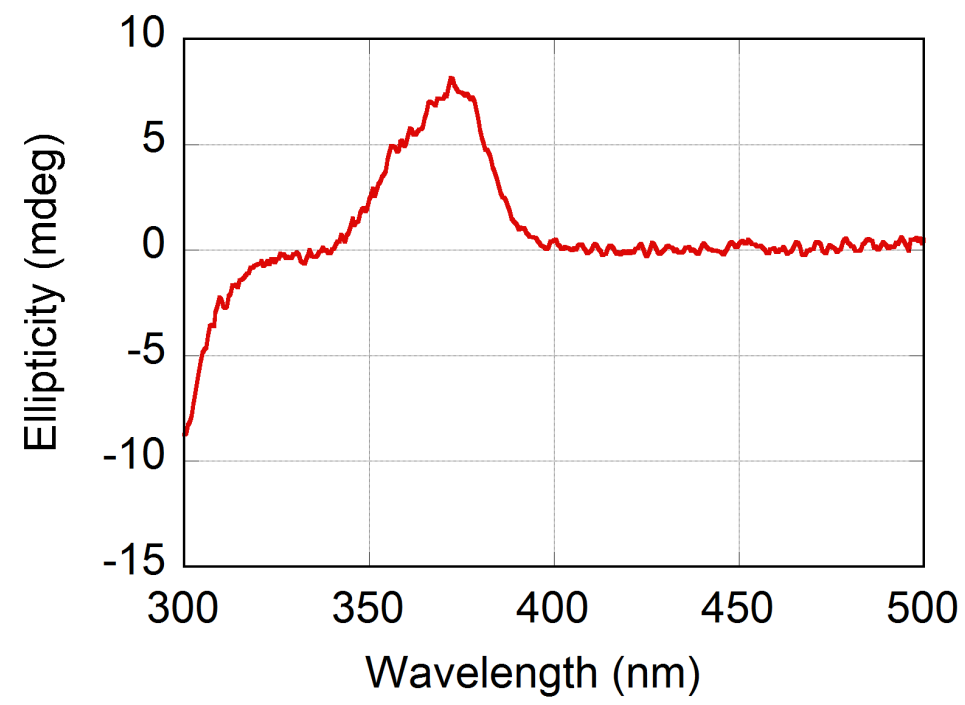

Figure S50. CD spectrum of $\mathbf{2 b}(\mathbf{5} / \mathbf{1 0 0})$ in $\mathrm{PhH}\left(2.48 \times 10^{-2} \mathrm{~g} / \mathrm{L}\right.$, light path length $=10$ $\mathrm{mm})$. 


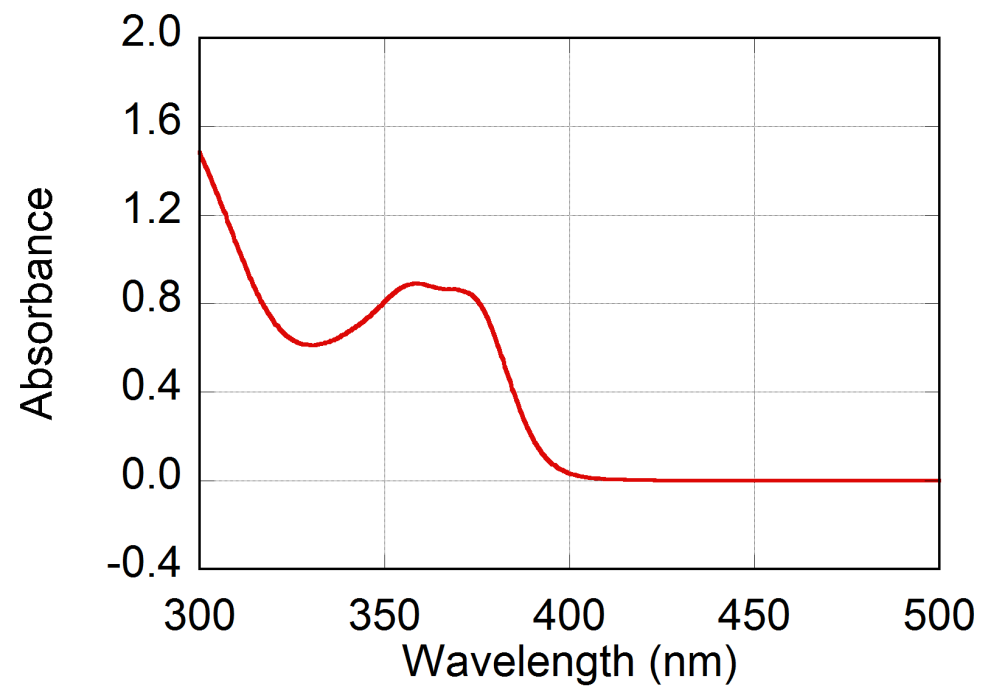

Figure S51. UV-vis absorption spectrum of $\mathbf{2 b}(\mathbf{5} / \mathbf{1 0 0})$ in $\mathrm{PhCH}_{3}\left(2.48 \times 10^{-2} \mathrm{~g} / \mathrm{L}\right.$, light path length $=10 \mathrm{~mm}$ ).

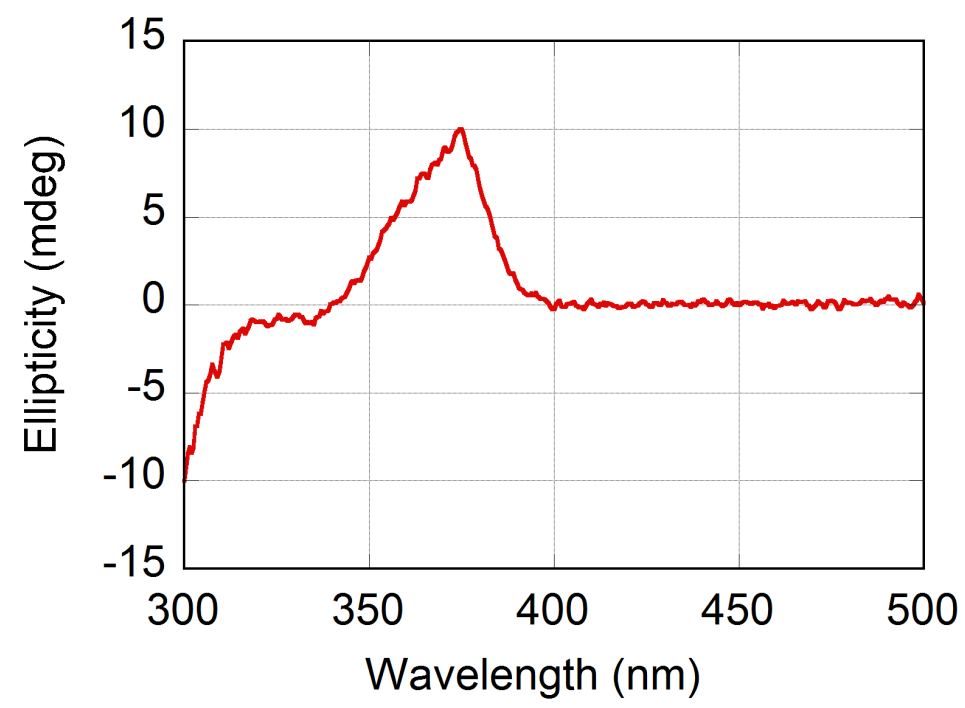

Figure S52. CD spectrum of $\mathbf{2 b}(\mathbf{5} / \mathbf{1 0 0})$ in $\mathrm{PhCH}_{3}\left(2.48 \times 10^{-2} \mathrm{~g} / \mathrm{L}\right.$, light path length $=$ $10 \mathrm{~mm}$ ). 


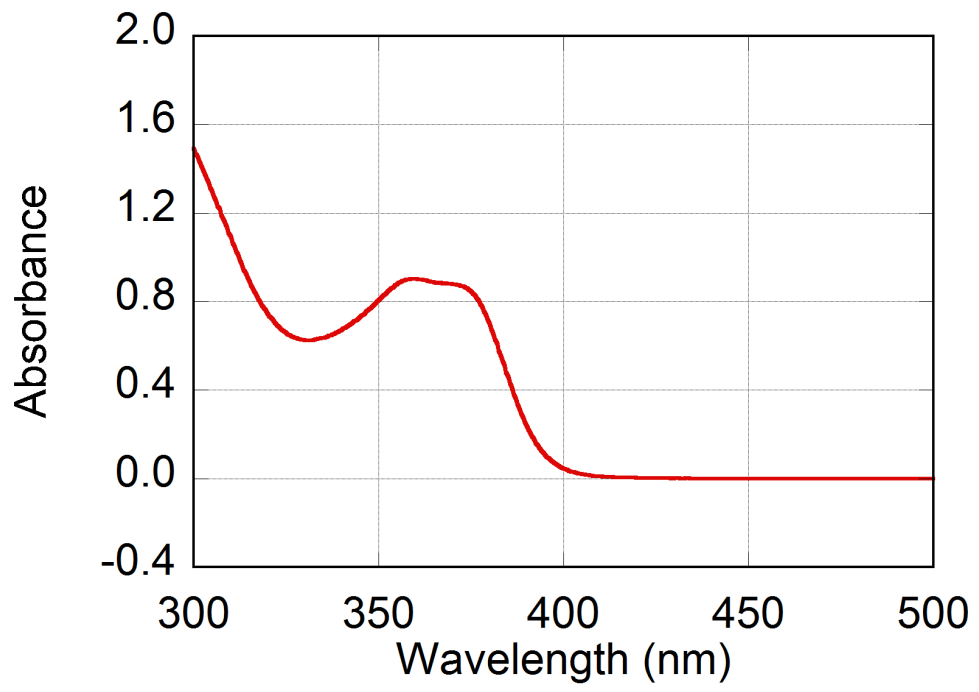

Figure S53. UV-vis absorption spectrum of $\mathbf{2 b}(\mathbf{5} / \mathbf{1 0 0})$ in $\mathrm{PhOCH}_{3}\left(2.48 \times 10^{-2} \mathrm{~g} / \mathrm{L}\right.$, light path length $=10 \mathrm{~mm}$ ).

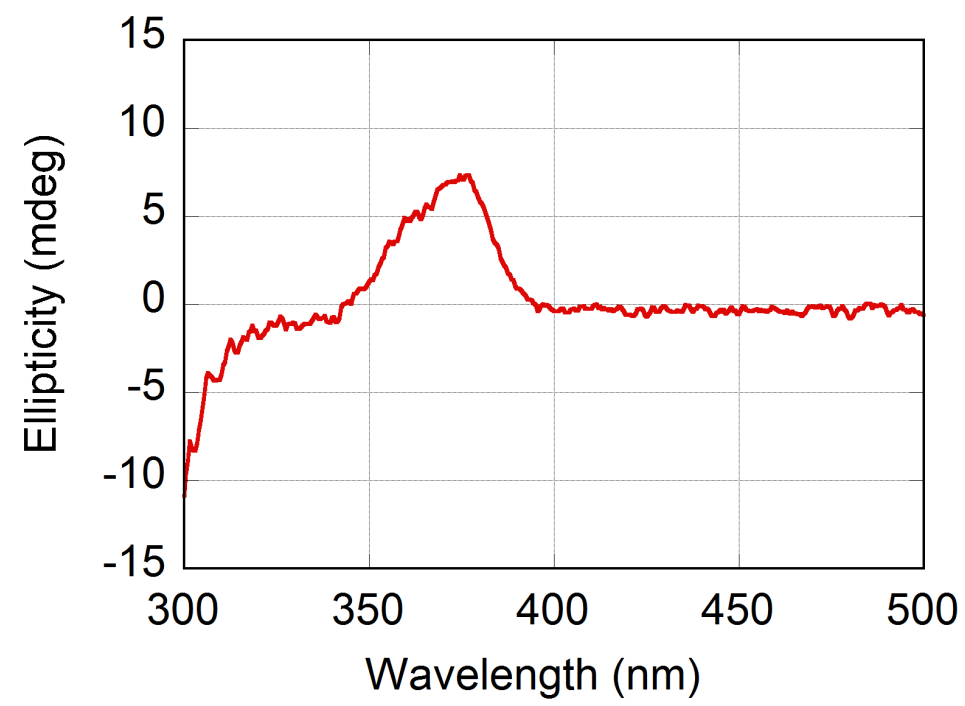

Figure S54. CD spectrum of $\mathbf{2 b}(\mathbf{5} / \mathbf{1 0 0})$ in $\mathrm{PhOCH}_{3}\left(2.48 \times 10^{-2} \mathrm{~g} / \mathrm{L}\right.$, light path length $=$ $10 \mathrm{~mm}$ ). 


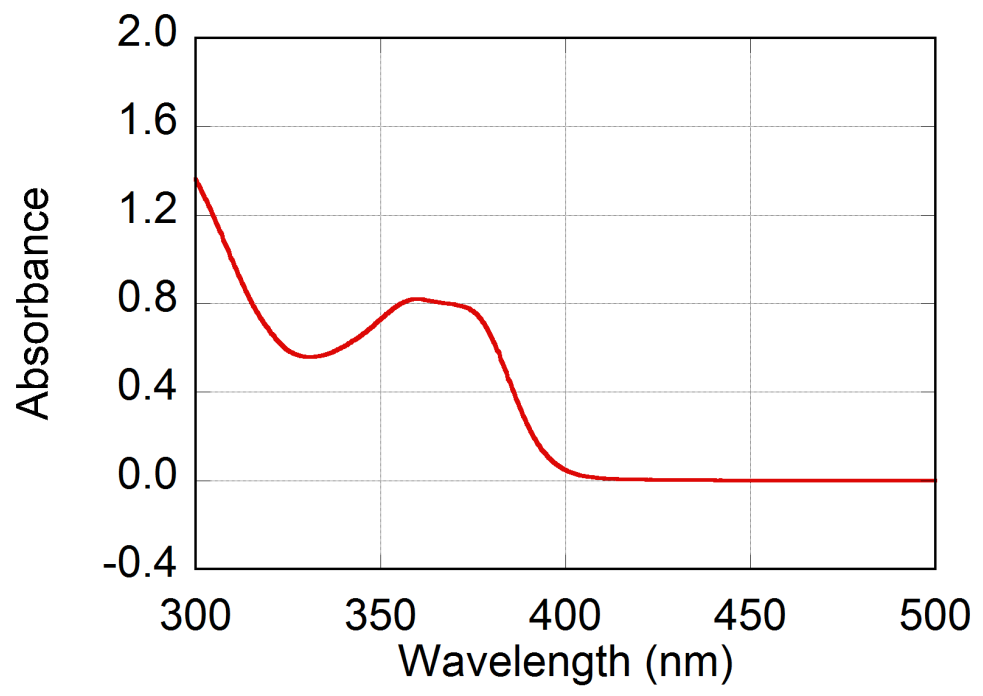

Figure S55. UV-vis absorption spectrum of $\mathbf{2 b}(\mathbf{5} / \mathbf{1 0 0})$ in $\mathrm{PhCF}_{3}\left(2.48 \times 10^{-2} \mathrm{~g} / \mathrm{L}\right.$, light path length $=10 \mathrm{~mm}$ ).

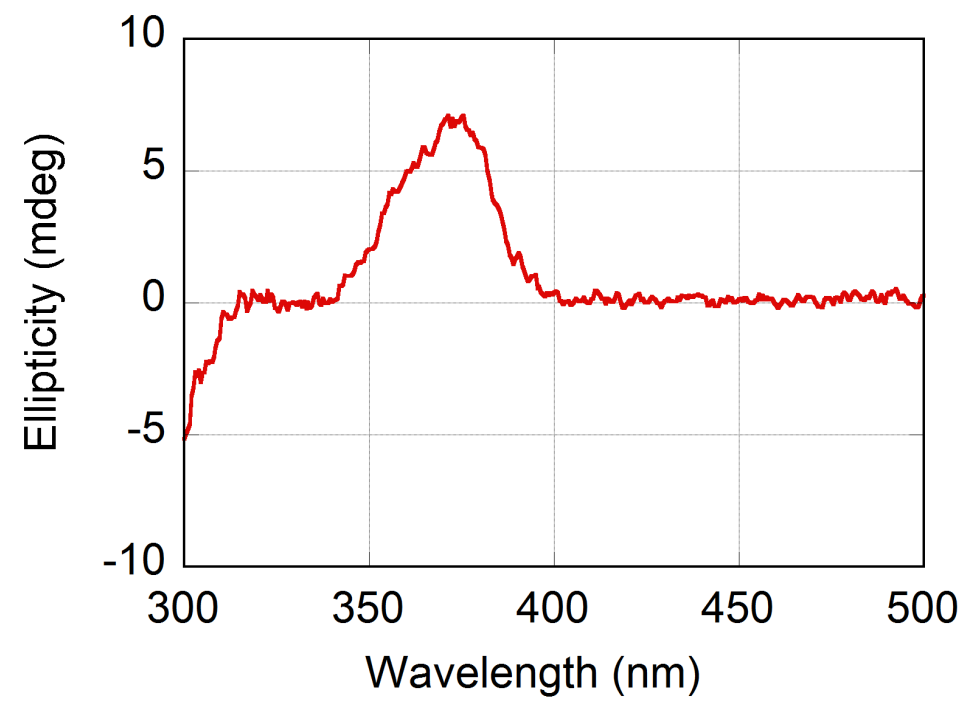

Figure S56. CD spectrum of $\mathbf{2 b}(\mathbf{5} / \mathbf{1 0 0})$ in $\mathrm{PhCF}_{3}\left(2.48 \times 10^{-2} \mathrm{~g} / \mathrm{L}\right.$, light path length $=10$ $\mathrm{mm})$. 


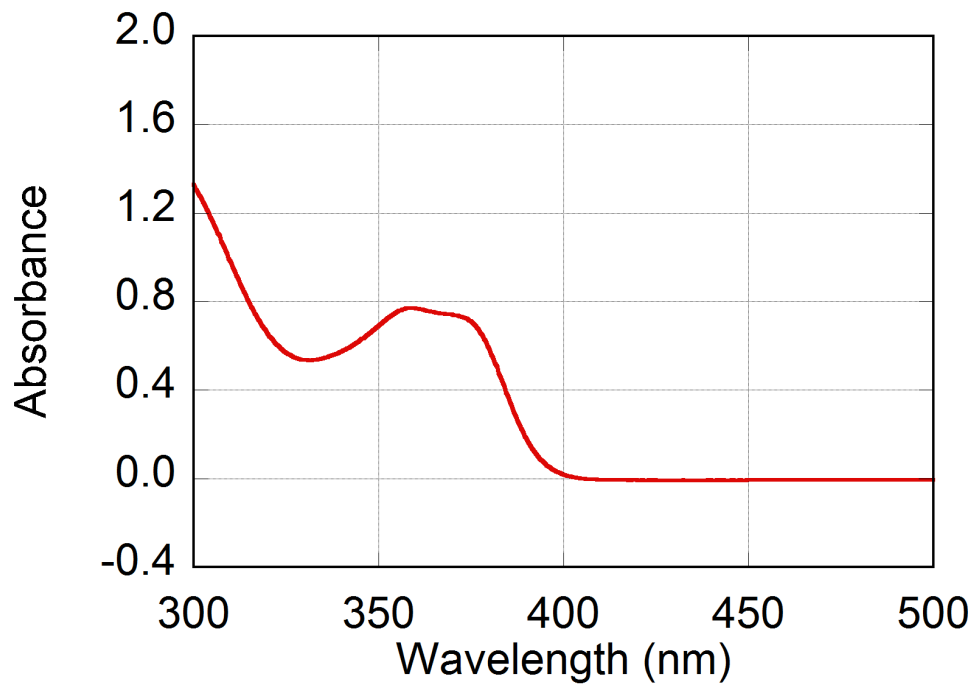

Figure S57. UV-vis absorption spectrum of $\mathbf{2 c ( 5 / 1 0 0 )}$ in $\mathrm{PhH}\left(2.97 \times 10^{-2} \mathrm{~g} / \mathrm{L}\right.$, light path length $=10 \mathrm{~mm}$ ).

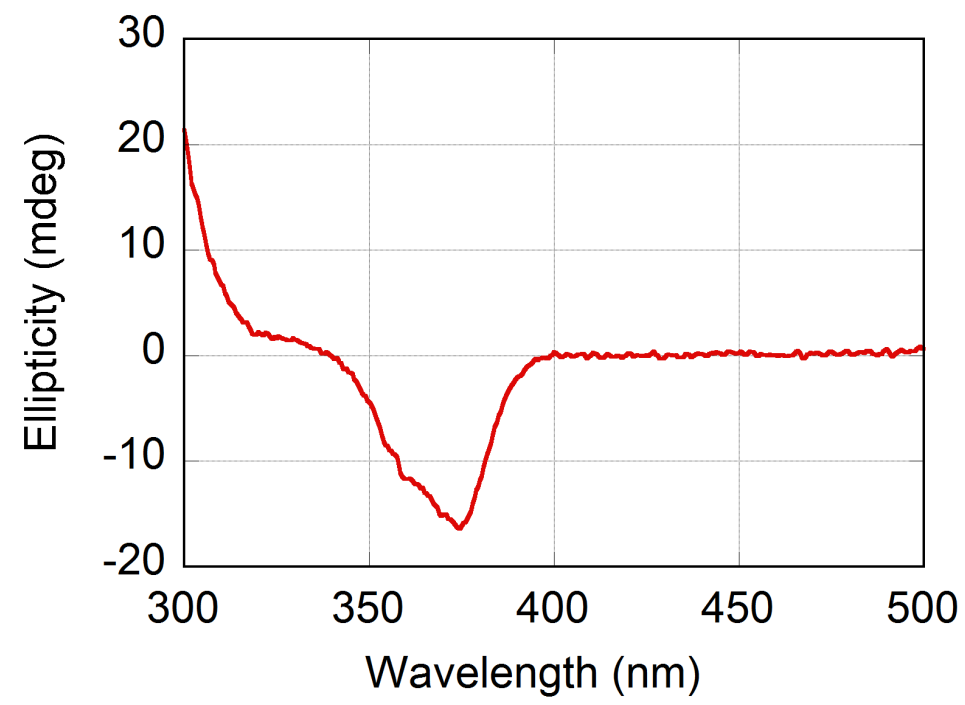

Figure S58. CD spectrum of $\mathbf{2 c ( 5 / 1 0 0 )}$ in $\mathrm{PhH}\left(2.97 \times 10^{-2} \mathrm{~g} / \mathrm{L}\right.$, light path length $=10$ $\mathrm{mm})$. 


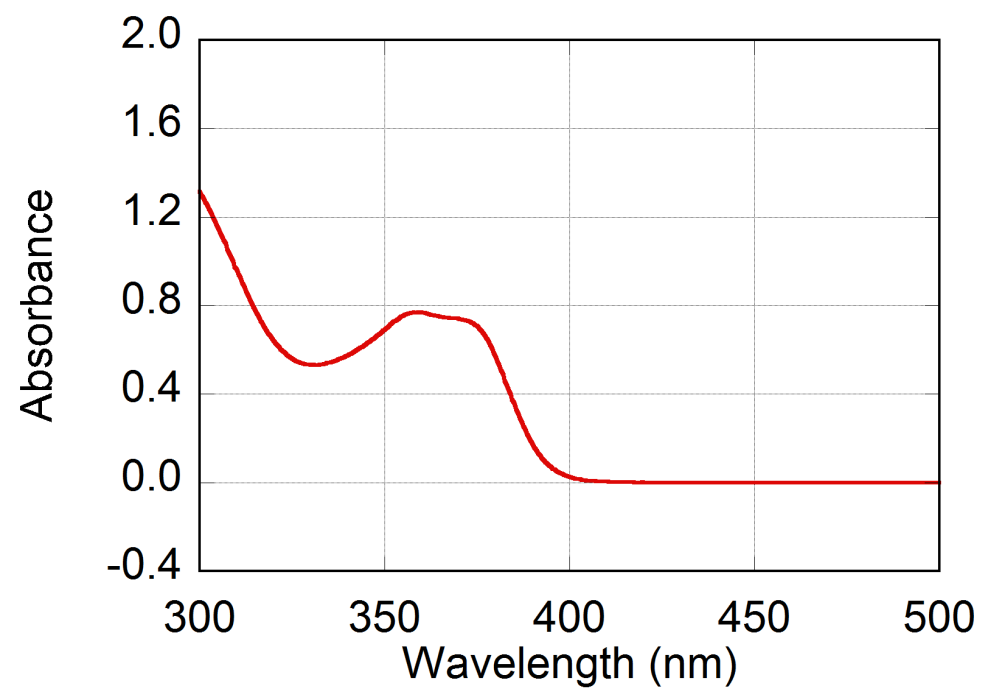

Figure S59. UV-vis absorption spectrum of 2c(5/100) in $\mathrm{PhCH}_{3}\left(2.97 \times 10^{-2} \mathrm{~g} / \mathrm{L}\right.$, light path length $=10 \mathrm{~mm}$ ).

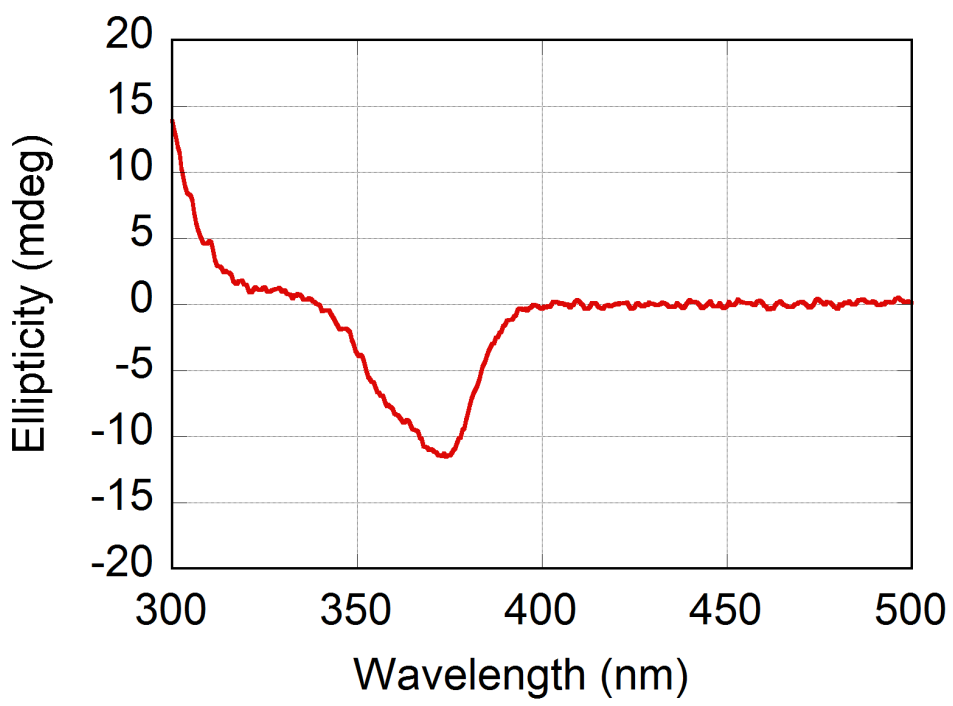

Figure S60. CD spectrum of $\mathbf{2 c ( 5 / 1 0 0 )}$ in $\mathrm{PhCH}_{3}\left(2.97 \times 10^{-2} \mathrm{~g} / \mathrm{L}\right.$, light path length $=$ $10 \mathrm{~mm}$ ). 


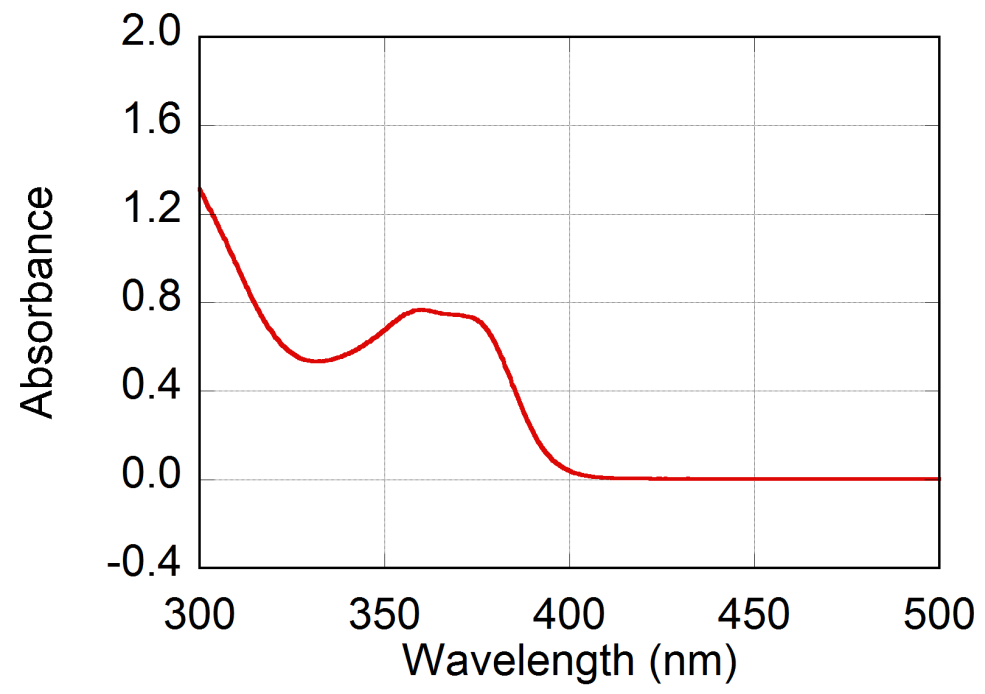

Figure S61. UV-vis absorption spectrum of $\mathbf{2 c ( 5 / 1 0 0 )}$ in $\mathrm{PhOCH}_{3}\left(2.97 \times 10^{-2} \mathrm{~g} / \mathrm{L}\right.$, light path length $=10 \mathrm{~mm}$ ).

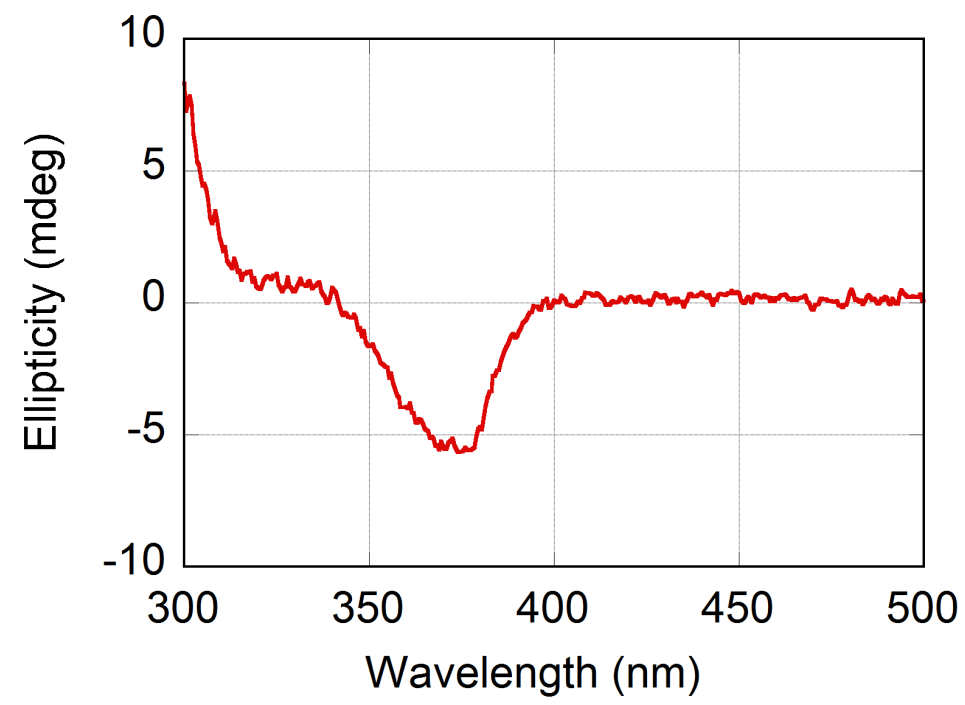

Figure S62. CD spectrum of $\mathbf{2 c ( 5 / 1 0 0 )}$ in $\mathrm{PhOCH}_{3}\left(2.97 \times 10^{-2} \mathrm{~g} / \mathrm{L}\right.$, light path length $=$ $10 \mathrm{~mm}$ ). 


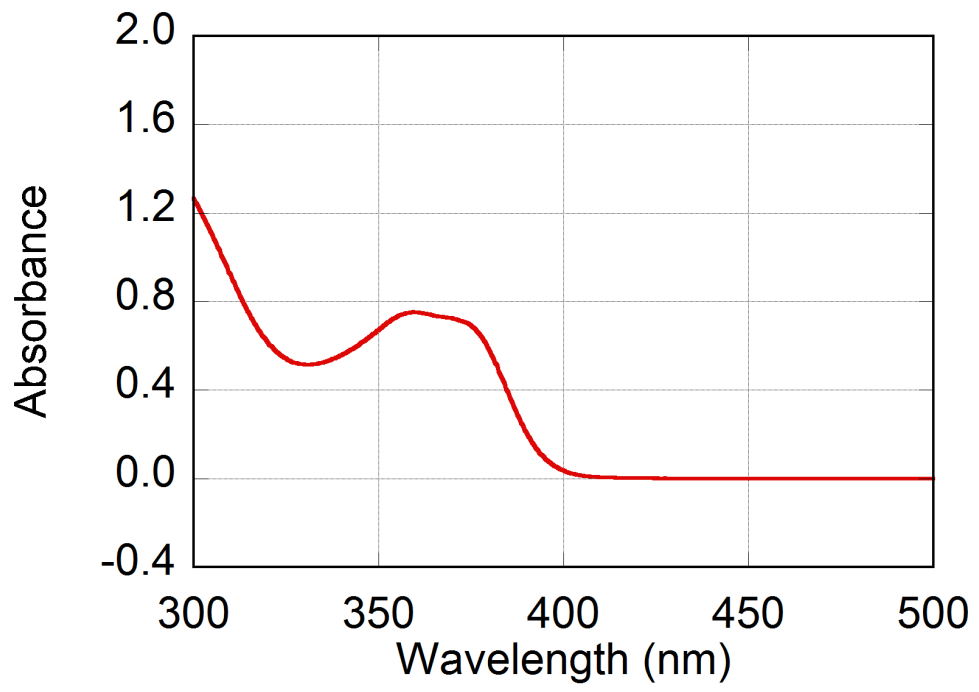

Figure S63. UV-vis absorption spectrum of 2c(5/100) in $\mathrm{PhCF}_{3}\left(2.97 \times 10^{-2} \mathrm{~g} / \mathrm{L}\right.$, light path length $=10 \mathrm{~mm}$ ).

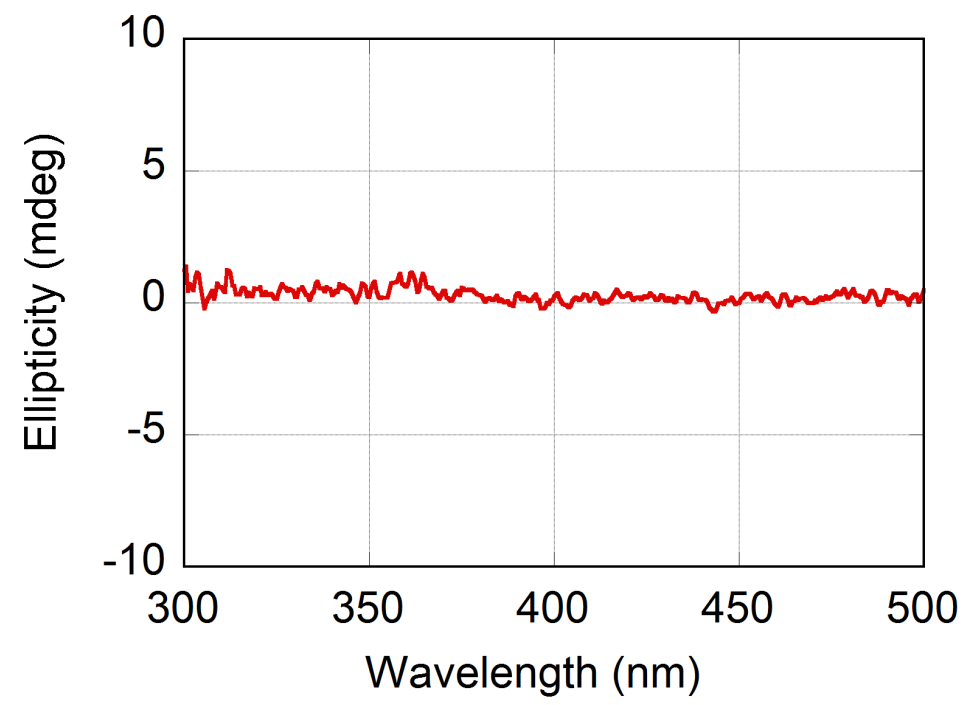

Figure S64. CD spectrum of $\mathbf{2 c ( 5 / 1 0 0 )}$ in $\mathrm{PhCF}_{3}\left(2.97 \times 10^{-2} \mathrm{~g} / \mathrm{L}\right.$, light path length $=10$ $\mathrm{mm})$. 


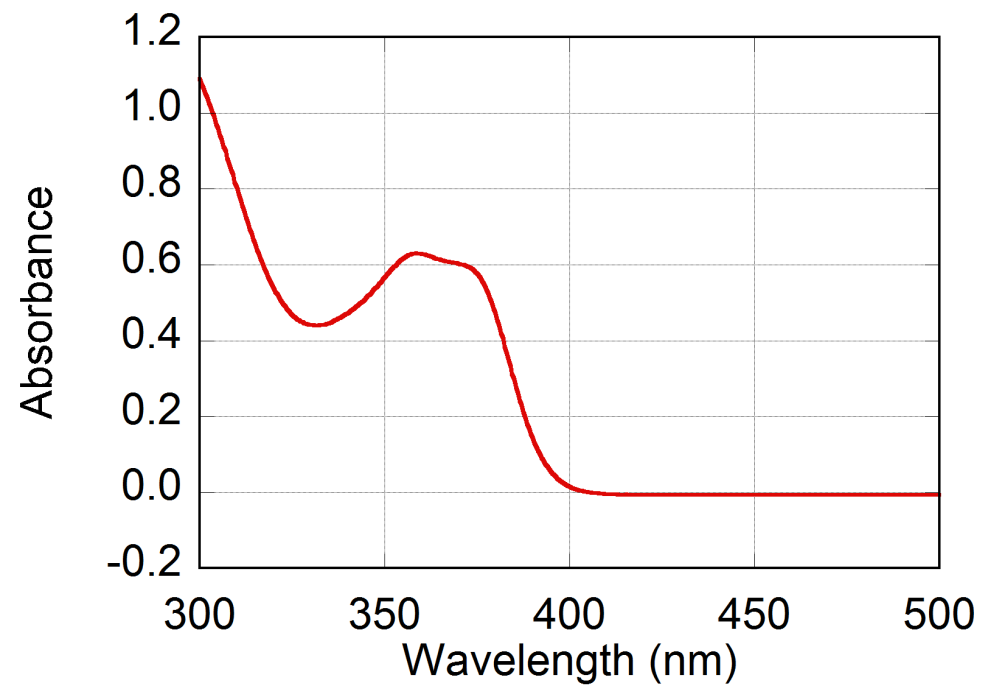

Figure S65. UV-vis absorption spectrum of 2d(5/100) in $\mathrm{PhH}\left(1.78 \times 10^{-2} \mathrm{~g} / \mathrm{L}\right.$, light path length $=10 \mathrm{~mm}$ ).

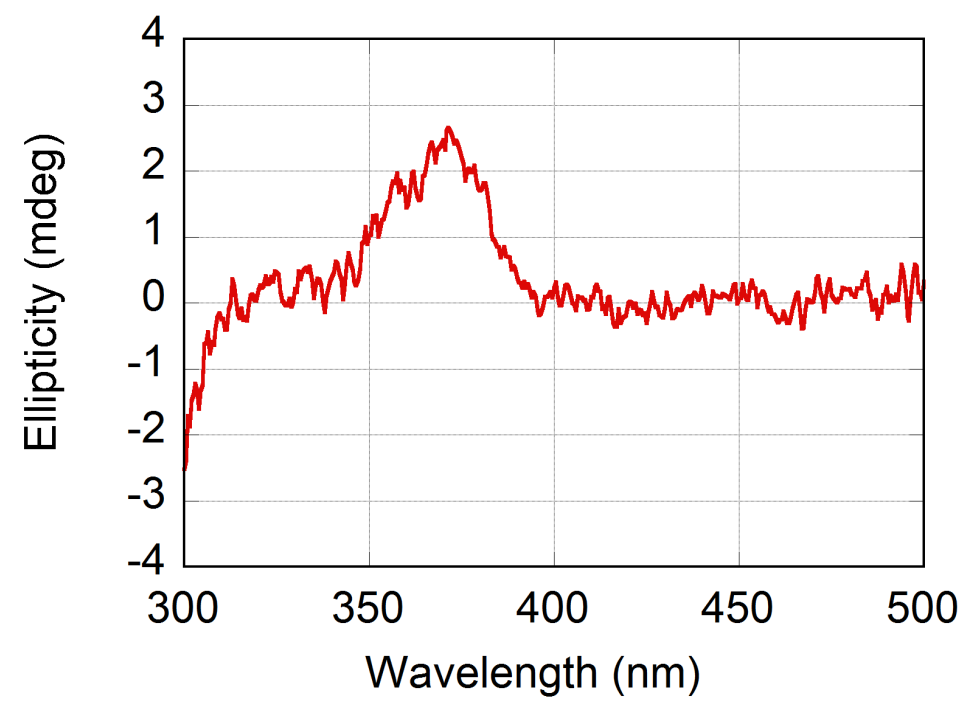

Figure S66. CD spectrum of $\mathbf{2 d ( 5 / 1 0 0 )}$ in $\mathrm{PhH}\left(1.78 \times 10^{-2} \mathrm{~g} / \mathrm{L}\right.$, light path length $=10$ $\mathrm{mm})$. 


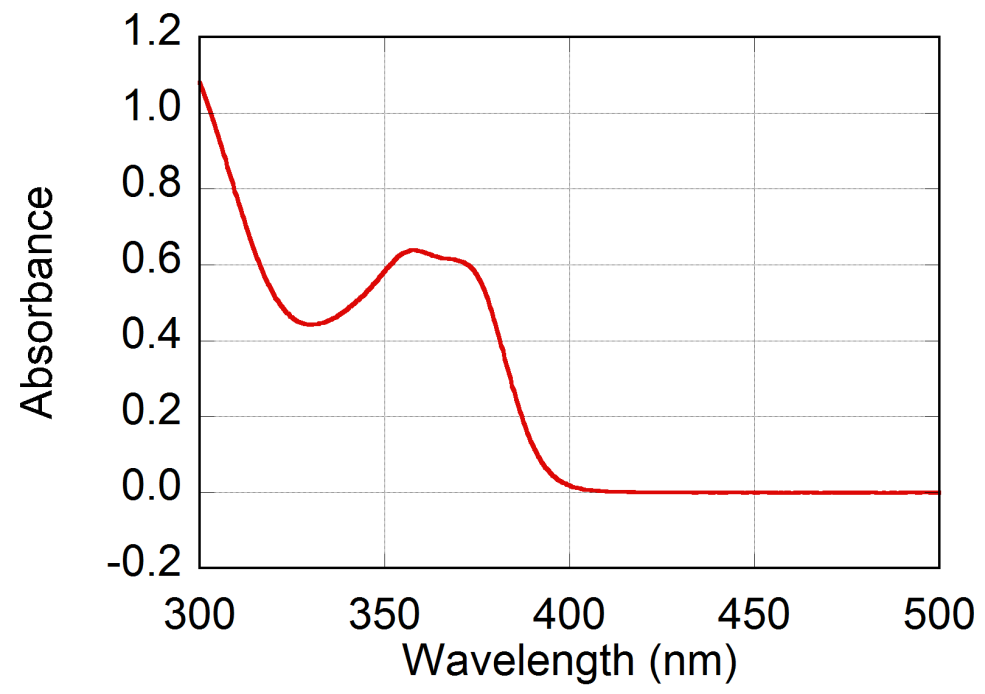

Figure S67. UV-vis absorption spectrum of 2d(5/100) in $\mathrm{PhCH}_{3}\left(1.78 \times 10^{-2} \mathrm{~g} / \mathrm{L}\right.$, light path length $=10 \mathrm{~mm}$ ).

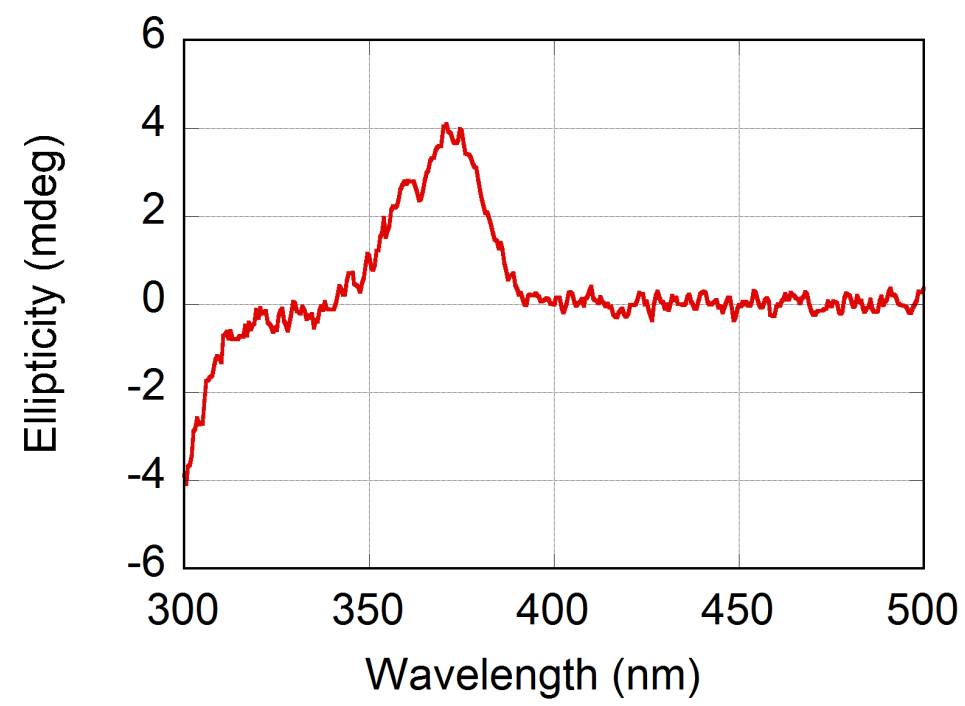

Figure S68. CD spectrum of $\mathbf{2 d ( 5 / 1 0 0 )}$ in $\mathrm{PhCH}_{3}\left(1.78 \times 10^{-2} \mathrm{~g} / \mathrm{L}\right.$, light path length $=$ $10 \mathrm{~mm}$ ). 


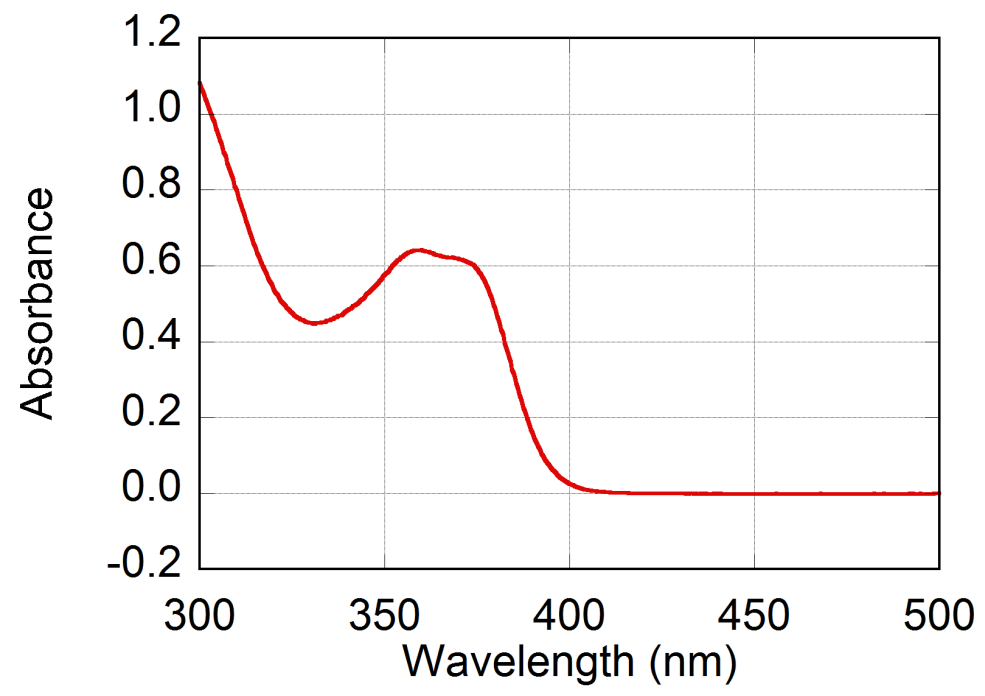

Figure S69. UV-vis absorption spectrum of 2d(5/100) in $\mathrm{PhOCH}_{3}\left(1.78 \times 10^{-2} \mathrm{~g} / \mathrm{L}\right.$, light path length $=10 \mathrm{~mm}$ ).

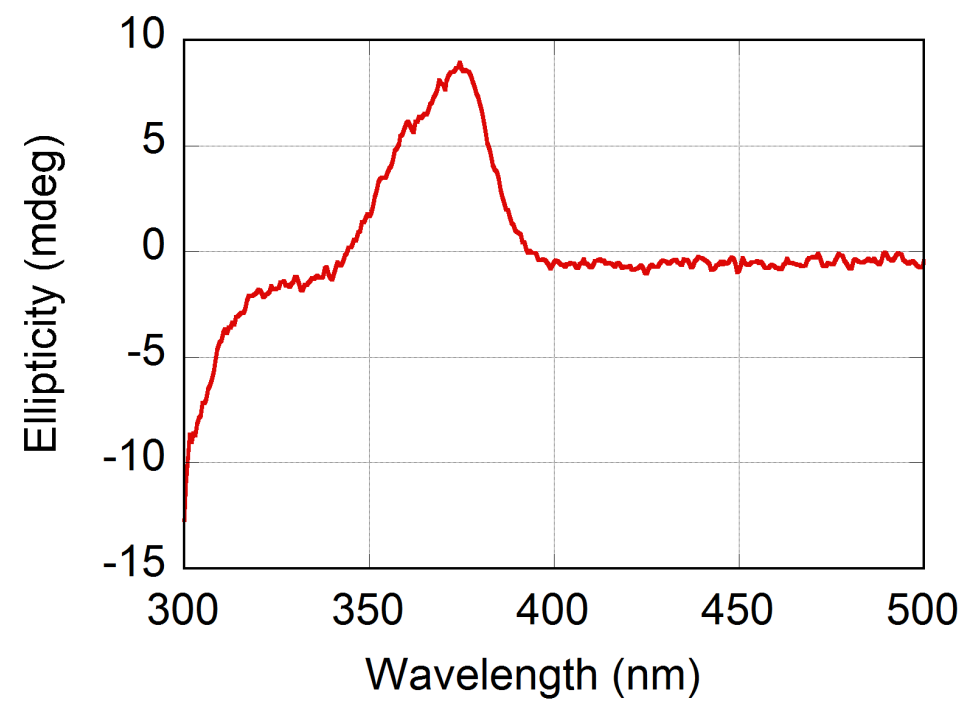

Figure S70. CD spectrum of $\mathbf{2 d ( 5 / 1 0 0 )}$ in $\mathrm{PhOCH}_{3}\left(1.78 \times 10^{-2} \mathrm{~g} / \mathrm{L}\right.$, light path length $=$ $10 \mathrm{~mm}$ ). 


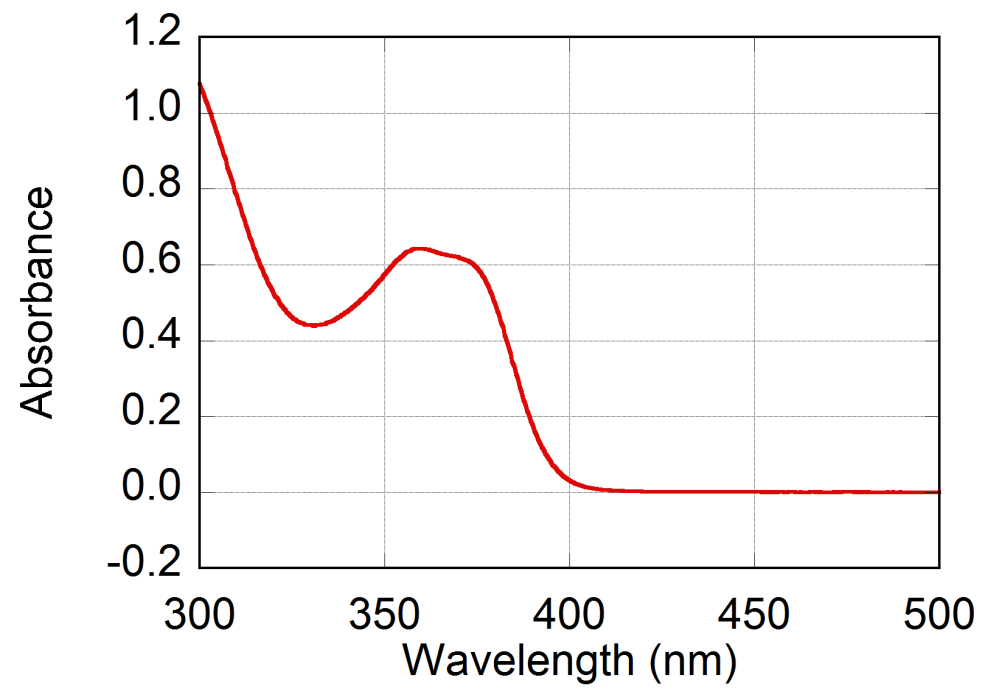

Figure S71. UV-vis absorption spectrum of 2d(5/100) in $\mathrm{PhCF}_{3}\left(1.78 \times 10^{-2} \mathrm{~g} / \mathrm{L}\right.$, light path length $=10 \mathrm{~mm}$ ).

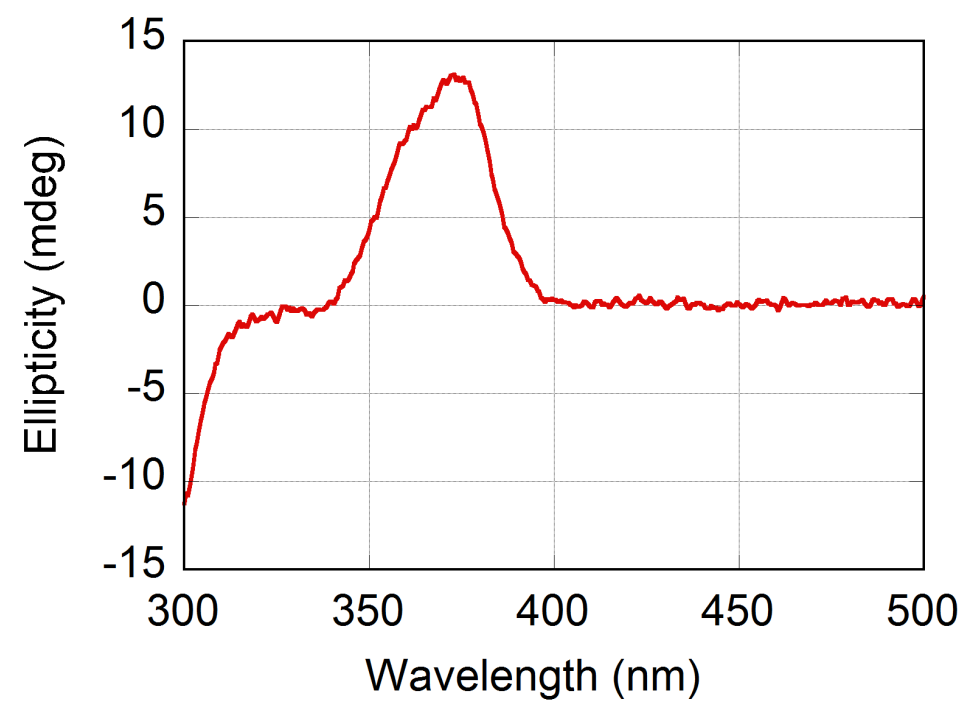

Figure S72. CD spectrum of $\mathbf{2 d ( 5 / 1 0 0 )}$ in $\mathrm{PhCF}_{3}\left(1.78 \times 10^{-2} \mathrm{~g} / \mathrm{L}\right.$, light path length $=10$ $\mathrm{mm})$. 


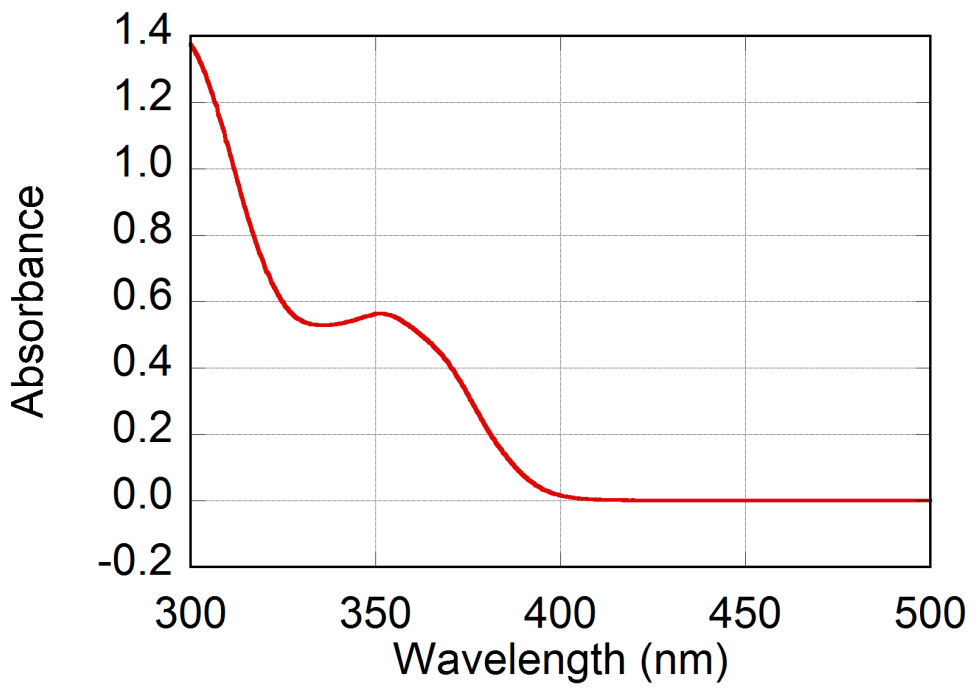

Figure S73. UV-vis absorption spectrum of 3a(5/100) in $\mathrm{PhH}\left(2.54 \times 10^{-2} \mathrm{~g} / \mathrm{L}\right.$, light path length $=10 \mathrm{~mm}$ ).

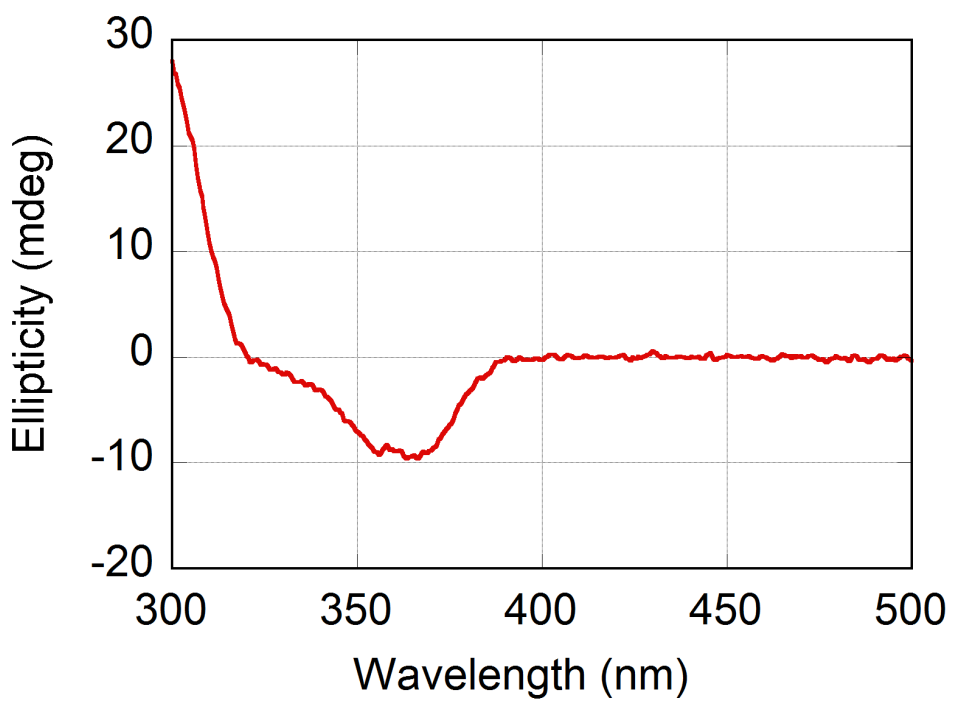

Figure S74. CD spectrum of $\mathbf{3 a}(\mathbf{5} / \mathbf{1 0 0})$ in $\mathrm{PhH}\left(2.54 \times 10^{-2} \mathrm{~g} / \mathrm{L}\right.$, light path length $=10$ $\mathrm{mm})$. 


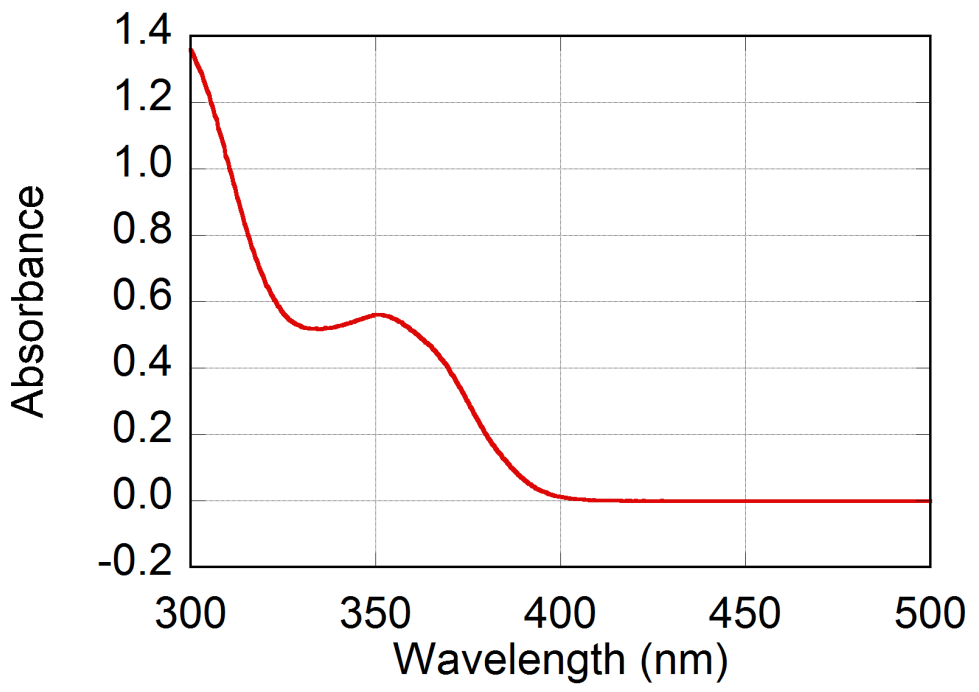

Figure S75. UV-vis absorption spectrum of $\mathbf{3 a ( 5 / 1 0 0 )}$ in $\mathrm{PhCH}_{3}\left(2.54 \times 10^{-2} \mathrm{~g} / \mathrm{L}\right.$, light path length $=10 \mathrm{~mm}$ ).

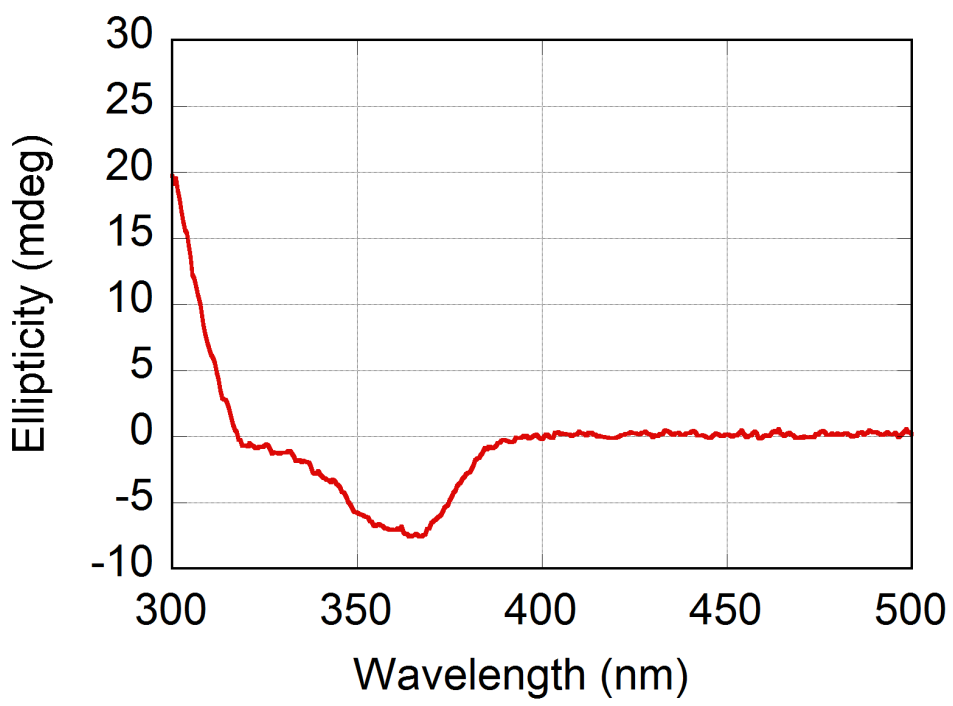

Figure S76. CD spectrum of $\mathbf{3 a ( 5 / 1 0 0 )}$ in $\mathrm{PhCH}_{3}\left(2.54 \times 10^{-2} \mathrm{~g} / \mathrm{L}\right.$, light path length $=$ $10 \mathrm{~mm})$. 


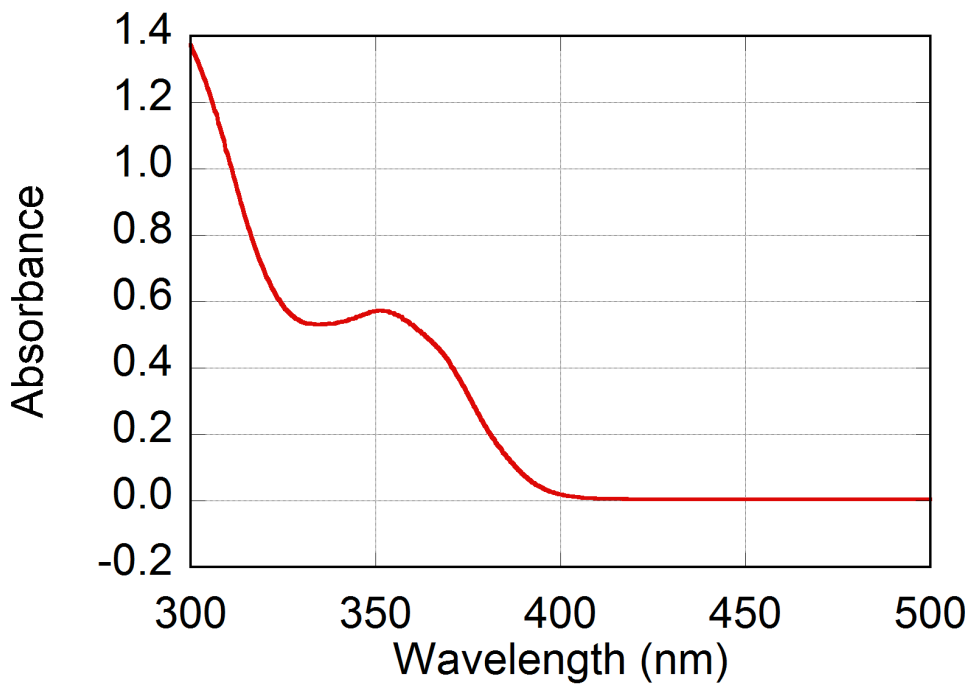

Figure S77. UV-vis absorption spectrum of 3a(5/100) in $\mathrm{PhOCH}_{3}\left(2.54 \times 10^{-2} \mathrm{~g} / \mathrm{L}\right.$, light path length $=10 \mathrm{~mm}$ ).

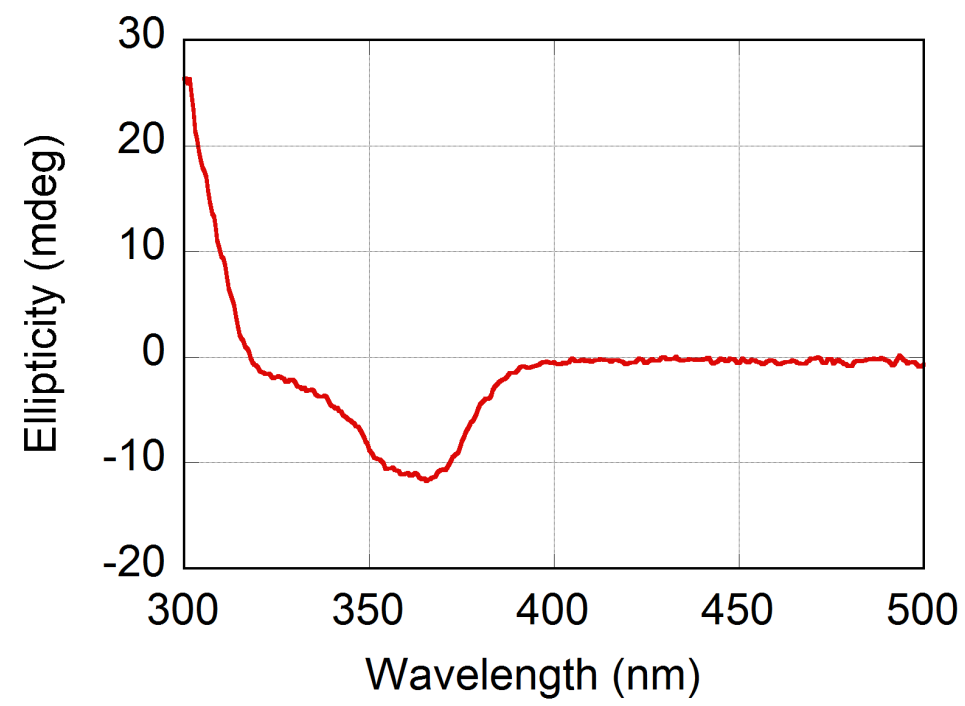

Figure S78. CD spectrum of $\mathbf{3 a}(\mathbf{5} / \mathbf{1 0 0})$ in $\mathrm{PhOCH}_{3}\left(2.54 \times 10^{-2} \mathrm{~g} / \mathrm{L}\right.$, light path length $=$ $10 \mathrm{~mm}$ ). 


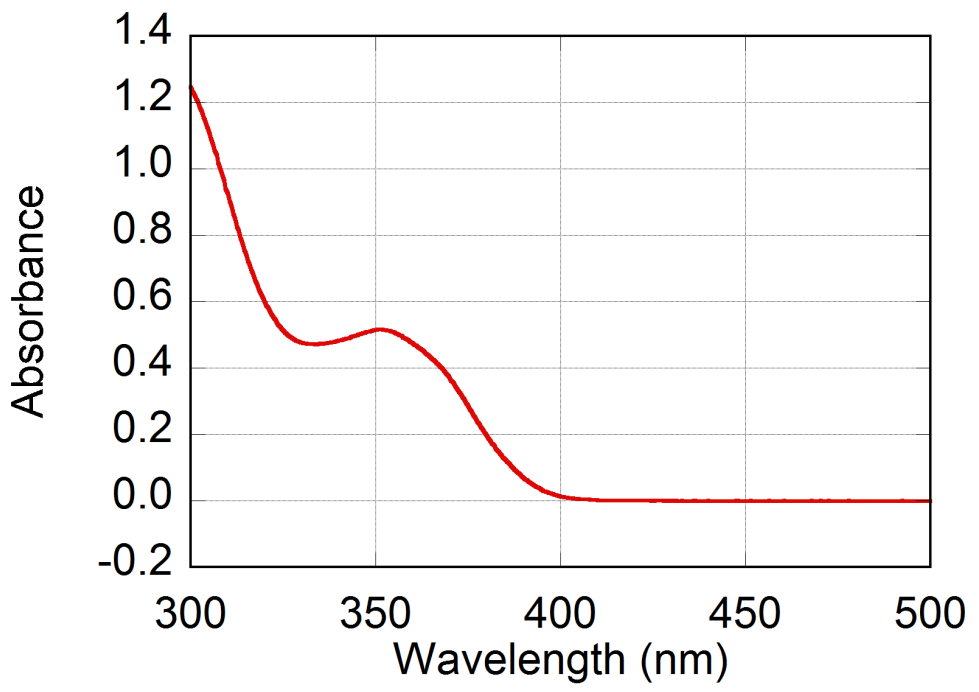

Figure S79. UV-vis absorption spectrum of 3a(5/100) in $\mathrm{PhCF}_{3}\left(2.54 \times 10^{-2} \mathrm{~g} / \mathrm{L}\right.$, light path length $=10 \mathrm{~mm}$ ).

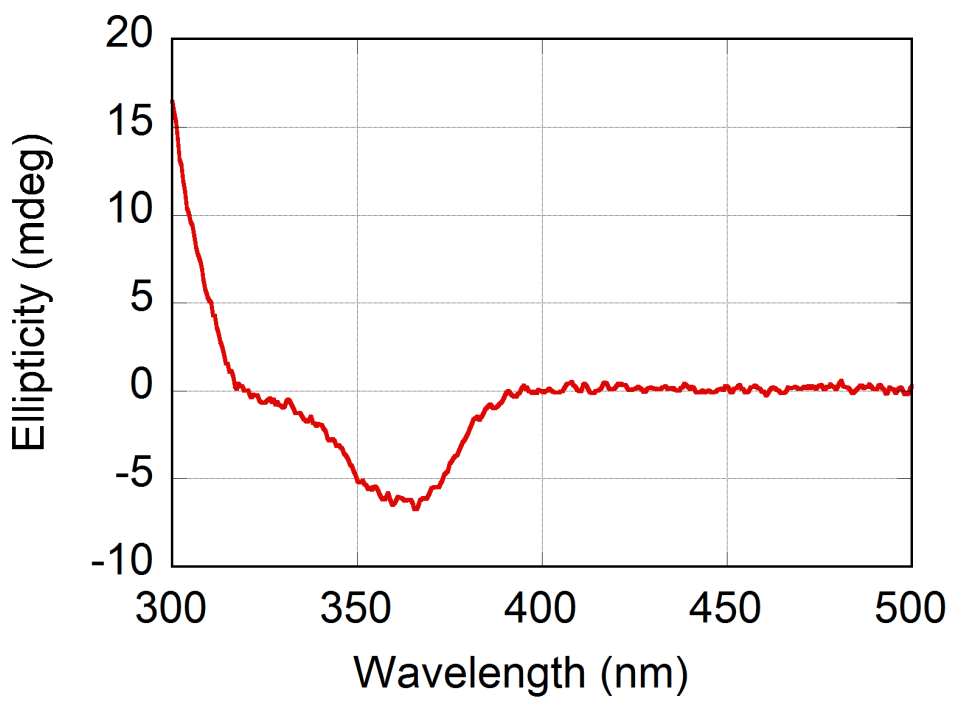

Figure S80. CD spectrum of $\mathbf{3 a ( 5 / 1 0 0})$ in $\mathrm{PhCF}_{3}\left(2.54 \times 10^{-2} \mathrm{~g} / \mathrm{L}\right.$, light path length $=10$ $\mathrm{mm})$. 


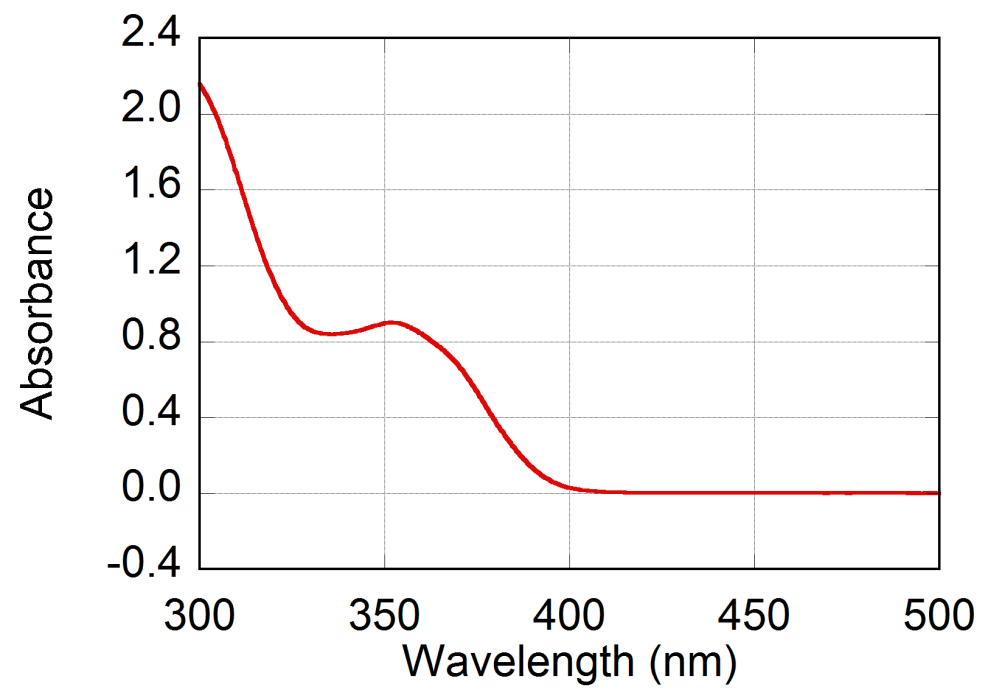

Figure S81. UV-vis absorption spectrum of $\mathbf{3 b}(\mathbf{5} / \mathbf{1 0 0})$ in $\mathrm{PhH}\left(3.64 \times 10^{-2} \mathrm{~g} / \mathrm{L}\right.$, light path length $=10 \mathrm{~mm}$ ).

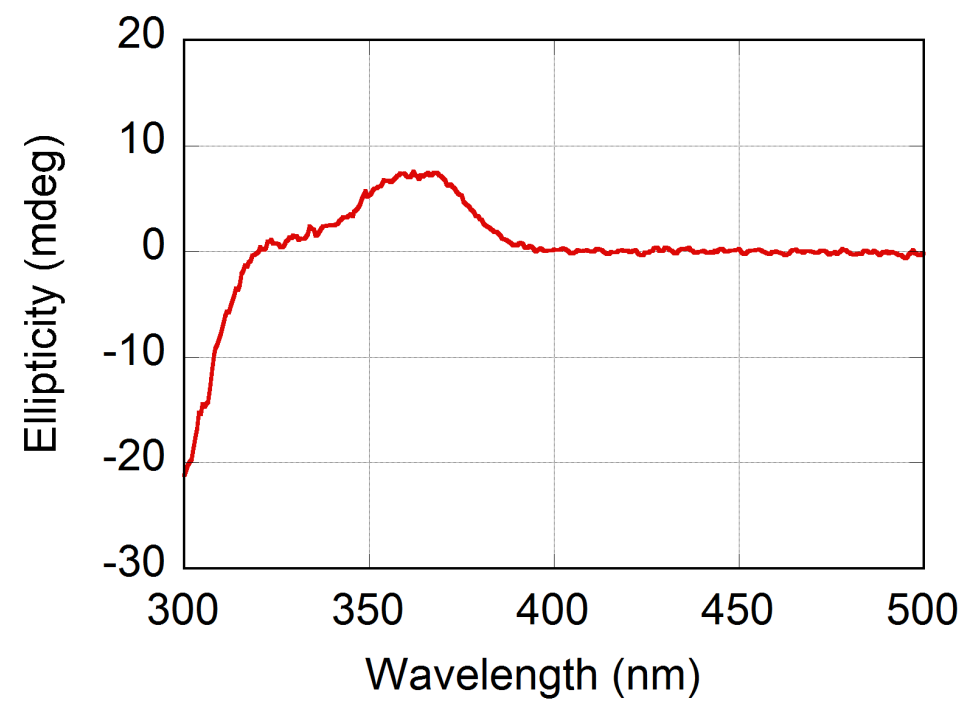

Figure S82. CD spectrum of $\mathbf{3 b}(\mathbf{5} / \mathbf{1 0 0})$ in $\mathrm{PhH}\left(3.64 \times 10^{-2} \mathrm{~g} / \mathrm{L}\right.$, light path length $=10$ $\mathrm{mm})$. 


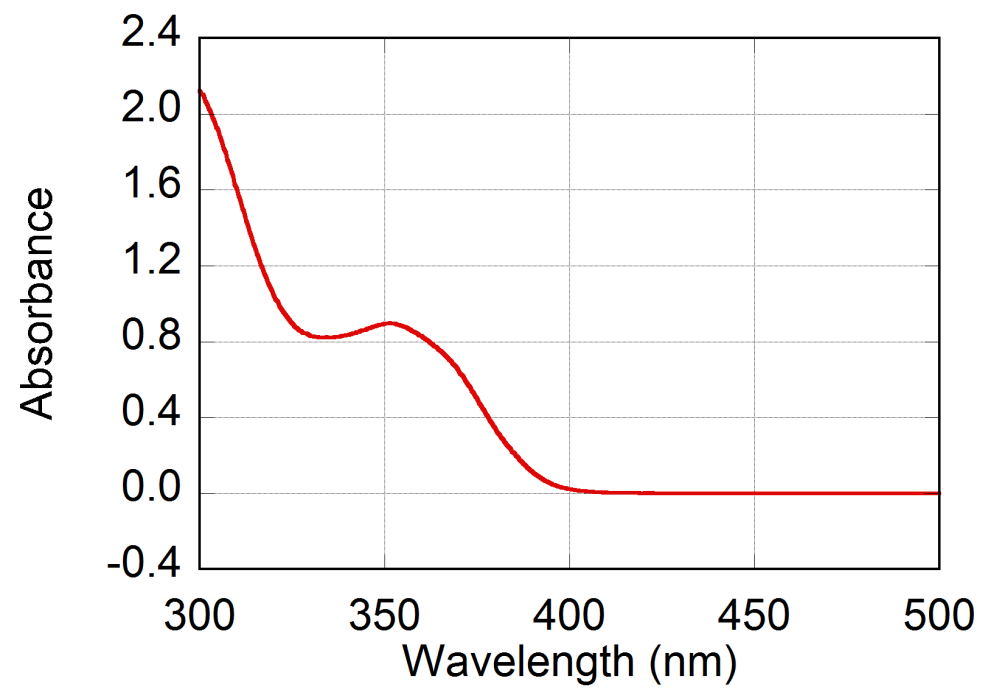

Figure S83. UV-vis absorption spectrum of $\mathbf{3 b} \mathbf{b} \mathbf{5} / \mathbf{1 0 0})$ in $\mathrm{PhCH}_{3}\left(3.64 \times 10^{-2} \mathrm{~g} / \mathrm{L}\right.$, light path length $=10 \mathrm{~mm}$ ).

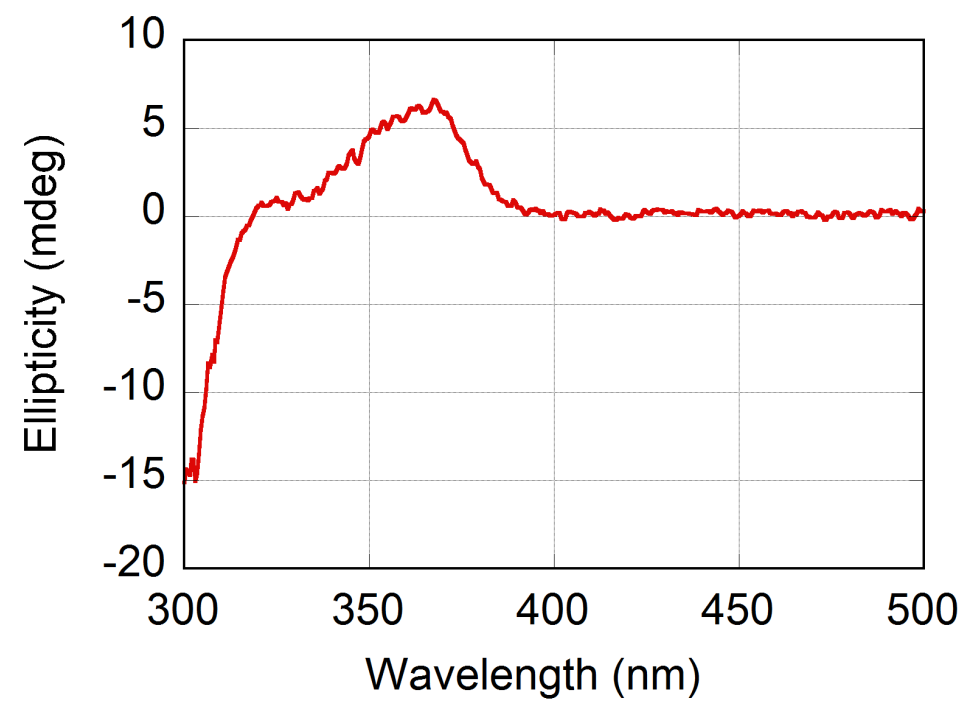

Figure S84. CD spectrum of $\mathbf{3 b}(\mathbf{5} / \mathbf{1 0 0})$ in $\mathrm{PhCH}_{3}\left(3.64 \times 10^{-2} \mathrm{~g} / \mathrm{L}\right.$, light path length $=$ $10 \mathrm{~mm}$ ). 


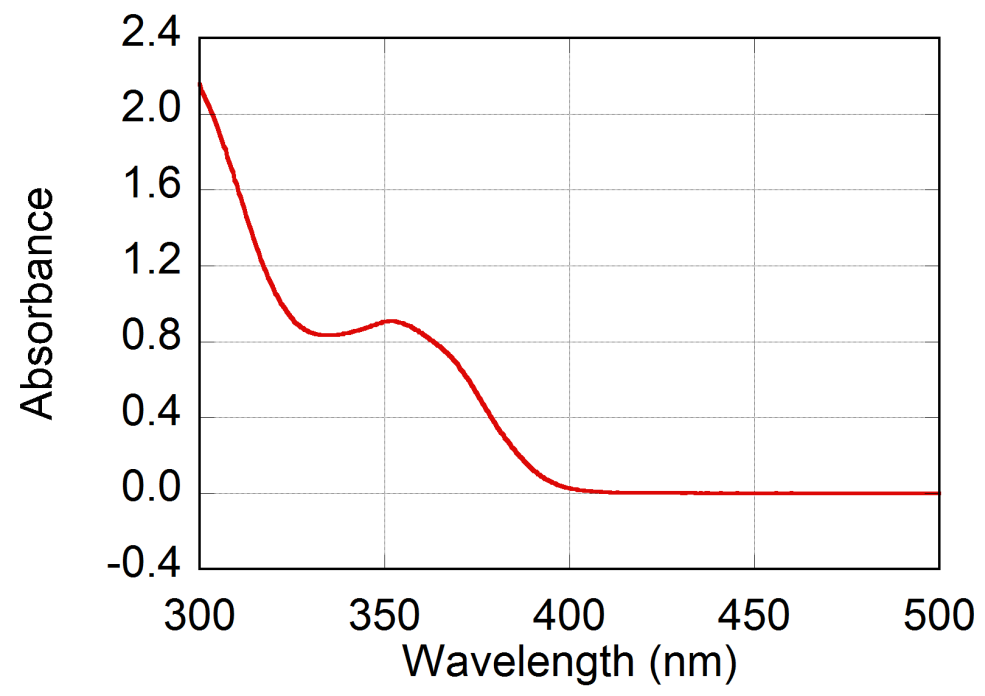

Figure S85. UV-vis absorption spectrum of 3b(5/100) in $\mathrm{PhOCH}_{3}\left(3.64 \times 10^{-2} \mathrm{~g} / \mathrm{L}\right.$, light path length $=10 \mathrm{~mm}$ ).

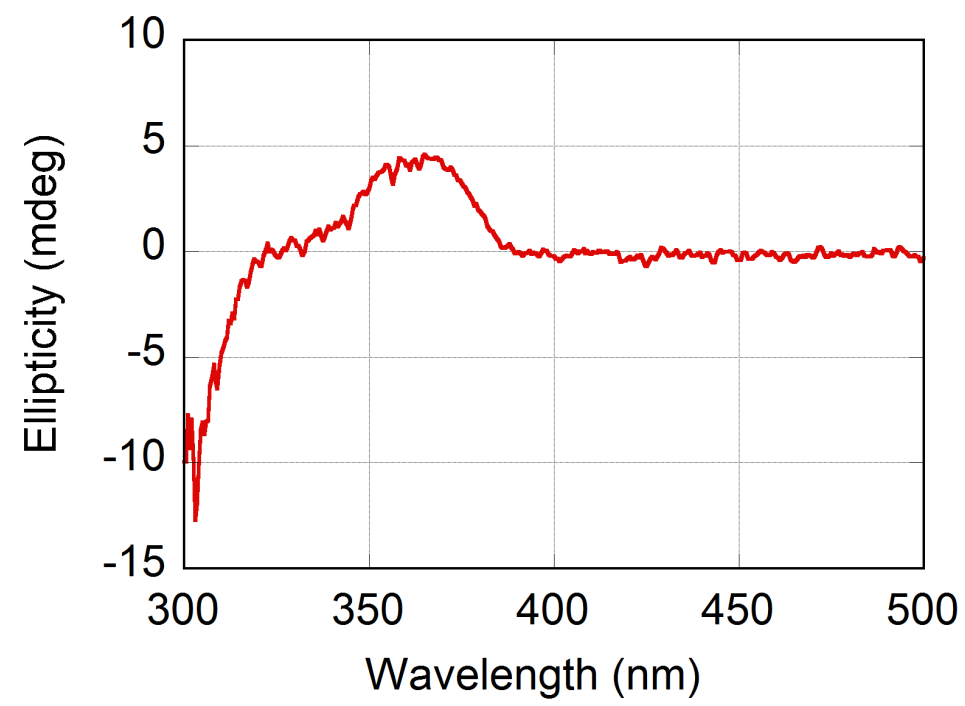

Figure S86. CD spectrum of $\mathbf{3 b}(\mathbf{5} / \mathbf{1 0 0})$ in $\mathrm{PhOCH}_{3}\left(3.64 \times 10^{-2} \mathrm{~g} / \mathrm{L}\right.$, light path length $=$ $10 \mathrm{~mm}$ ). 


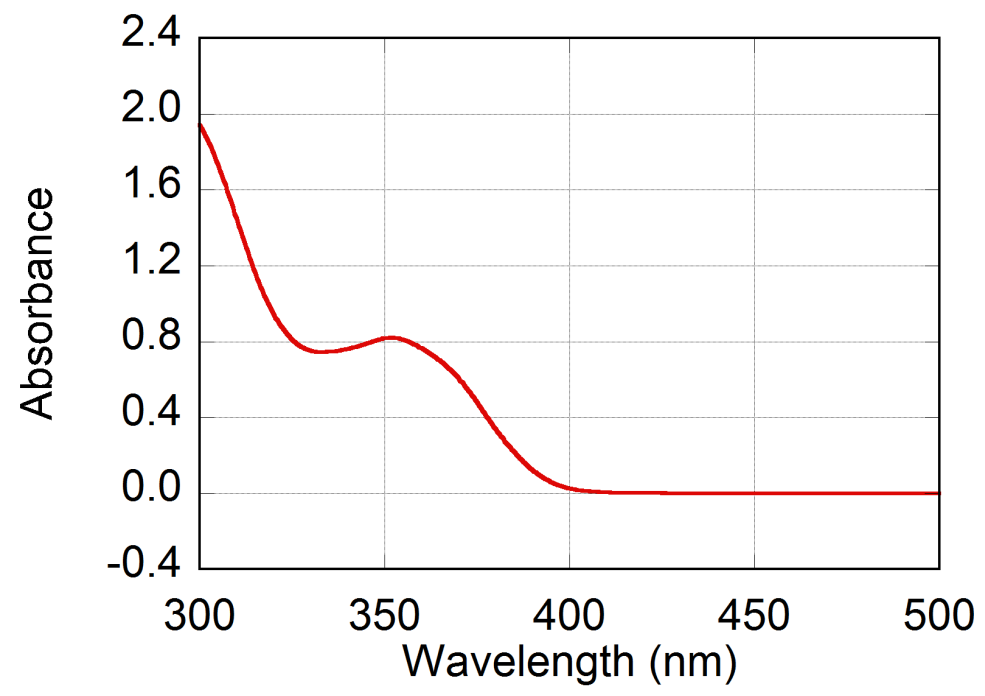

Figure S87. UV-vis absorption spectrum of $\mathbf{3 b}(\mathbf{5} / \mathbf{1 0 0})$ in $\mathrm{PhCF}_{3}\left(3.64 \times 10^{-2} \mathrm{~g} / \mathrm{L}\right.$, light path length $=10 \mathrm{~mm}$ ).

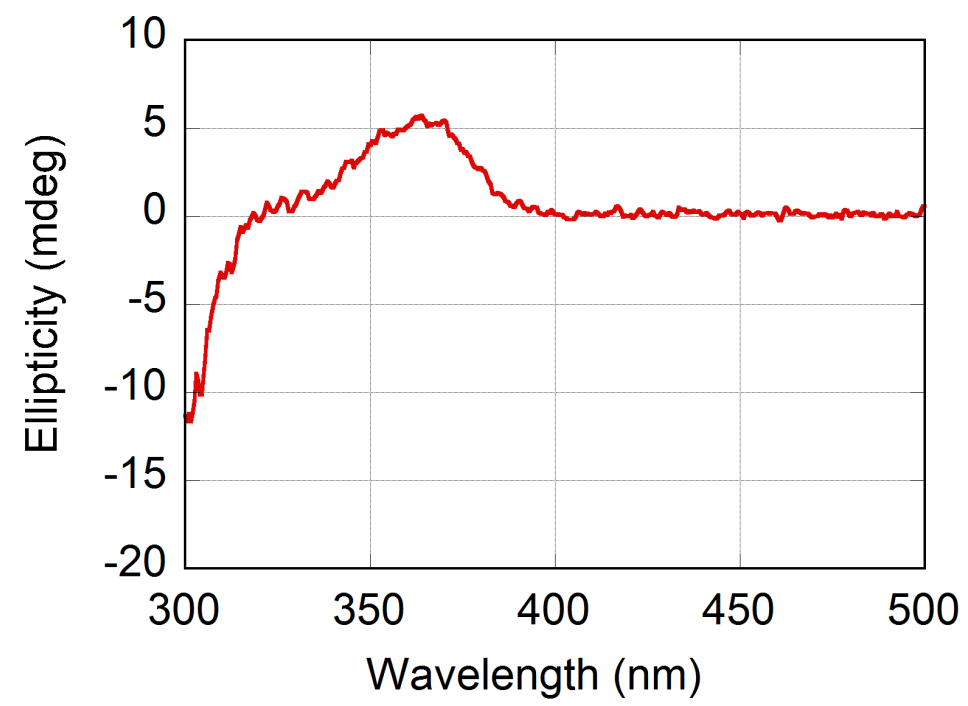

Figure S88. CD spectrum of $\mathbf{3 b}(\mathbf{5} / \mathbf{1 0 0})$ in $\mathrm{PhCF}_{3}\left(3.64 \times 10^{-2} \mathrm{~g} / \mathrm{L}\right.$, light path length $=10$ $\mathrm{mm})$. 


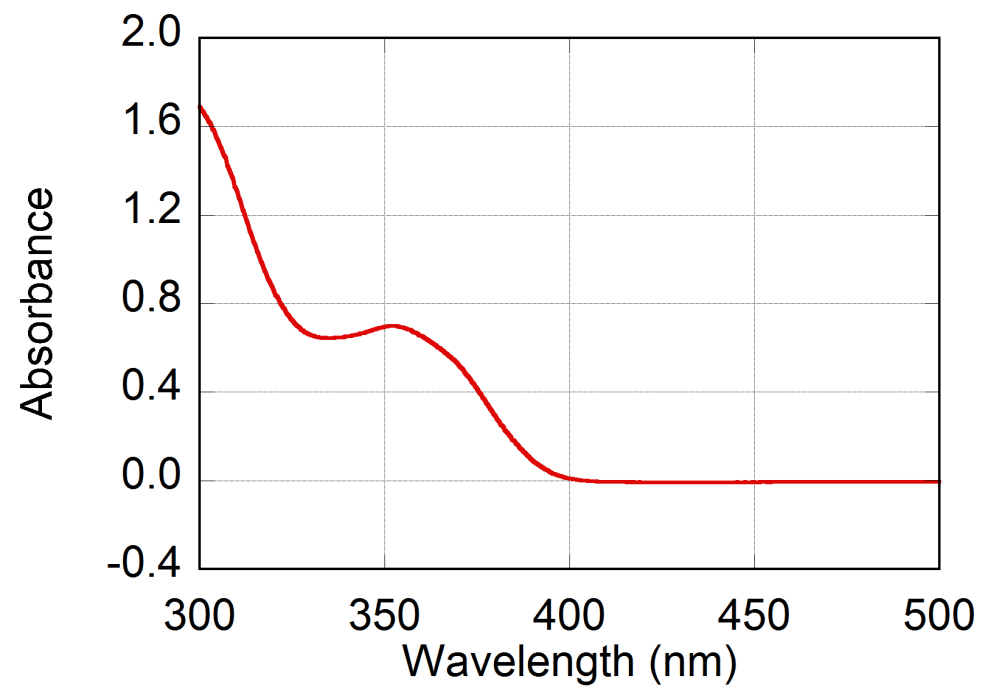

Figure S89. UV-vis absorption spectrum of 3c(5/100) in $\mathrm{PhH}\left(3.19 \times 10^{-2} \mathrm{~g} / \mathrm{L}\right.$, light path length $=10 \mathrm{~mm}$ ).

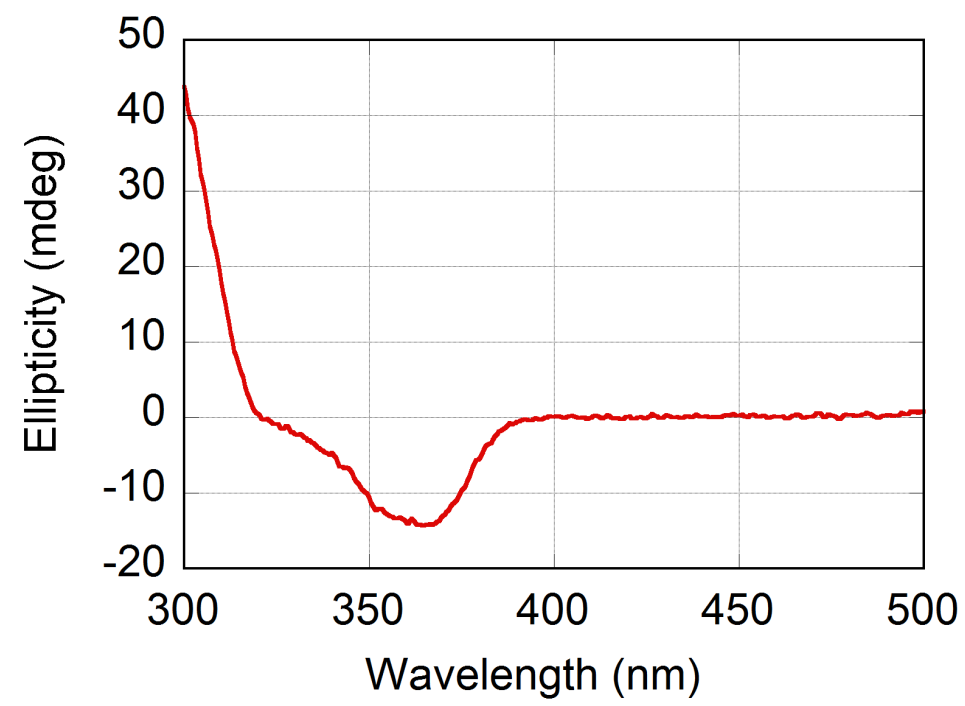

Figure S90. CD spectrum of $\mathbf{3 c}(\mathbf{5} / \mathbf{1 0 0})$ in $\mathrm{PhH}\left(3.19 \times 10^{-2} \mathrm{~g} / \mathrm{L}\right.$, light path length $=10$ $\mathrm{mm})$. 


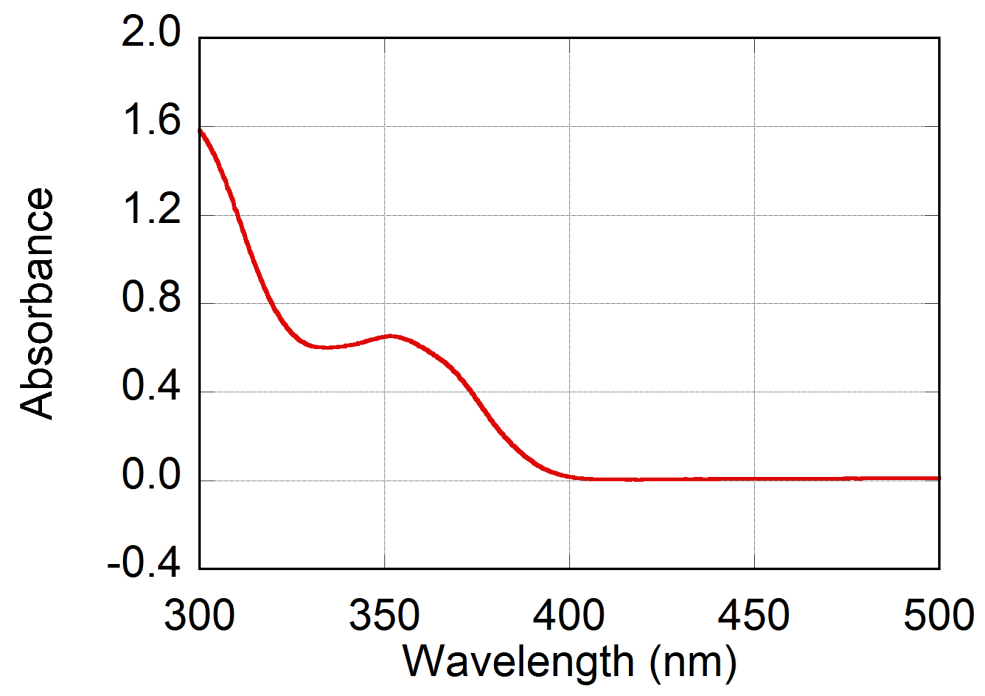

Figure S91. UV-vis absorption spectrum of $\mathbf{3 c}(\mathbf{5} / \mathbf{1 0 0})$ in $\mathrm{PhCH}_{3}\left(3.19 \times 10^{-2} \mathrm{~g} / \mathrm{L}\right.$, light path length $=10 \mathrm{~mm}$ ).

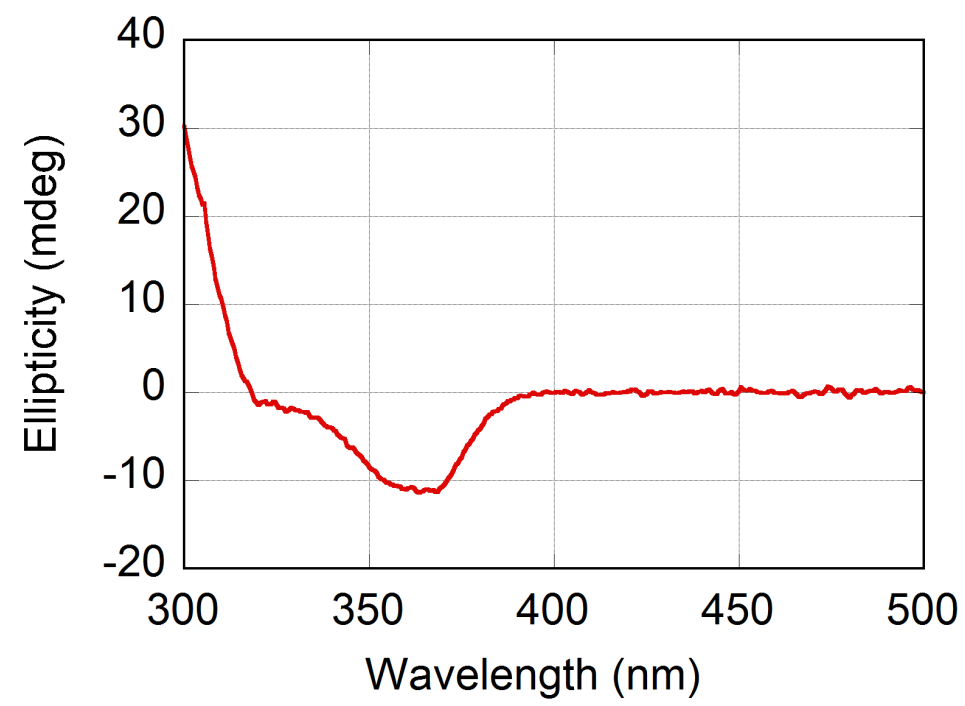

Figure S92. CD spectrum of $\mathbf{3 c}(\mathbf{5} / \mathbf{1 0 0})$ in $\mathrm{PhCH}_{3}\left(3.19 \times 10^{-2} \mathrm{~g} / \mathrm{L}\right.$, light path length $=$ $10 \mathrm{~mm}$ ). 


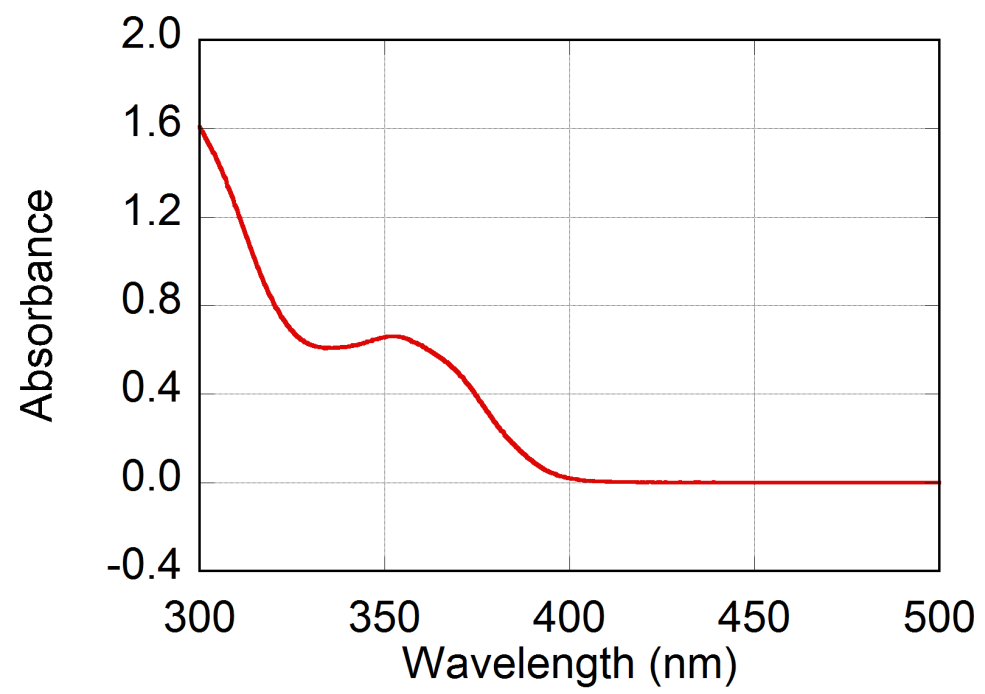

Figure S93. UV-vis absorption spectrum of $\mathbf{3 c}(\mathbf{5} / \mathbf{1 0 0})$ in $\mathrm{PhOCH}_{3}\left(3.19 \times 10^{-2} \mathrm{~g} / \mathrm{L}\right.$, light path length $=10 \mathrm{~mm}$ ).

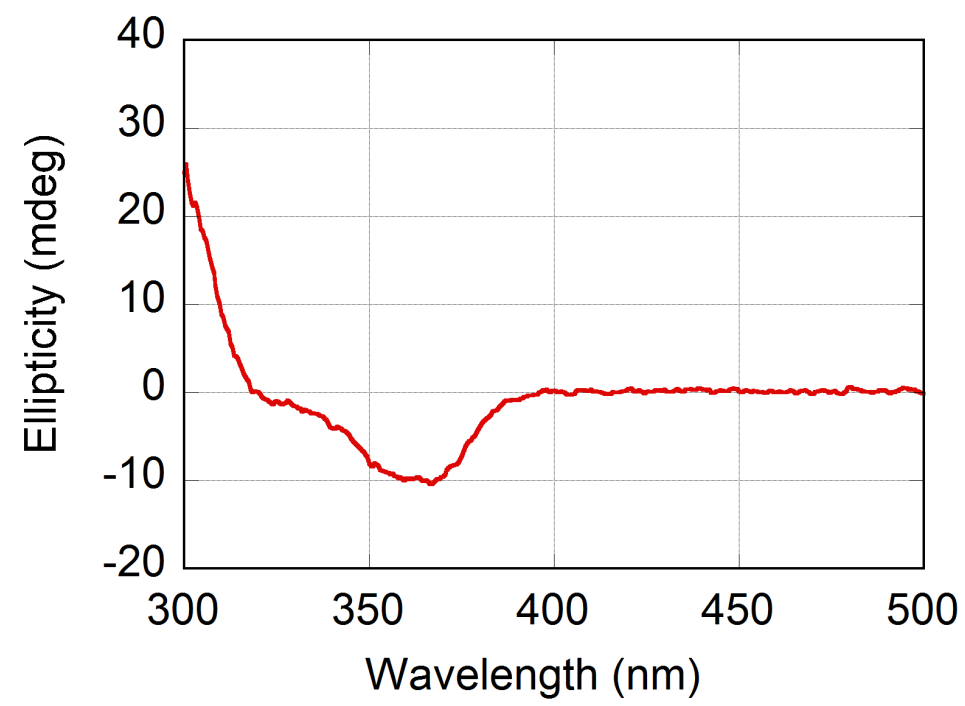

Figure S94. CD spectrum of $\mathbf{3 c}(\mathbf{5} / \mathbf{1 0 0})$ in $\mathrm{PhOCH}_{3}\left(3.19 \times 10^{-2} \mathrm{~g} / \mathrm{L}\right.$, light path length $=$ $10 \mathrm{~mm}$ ). 


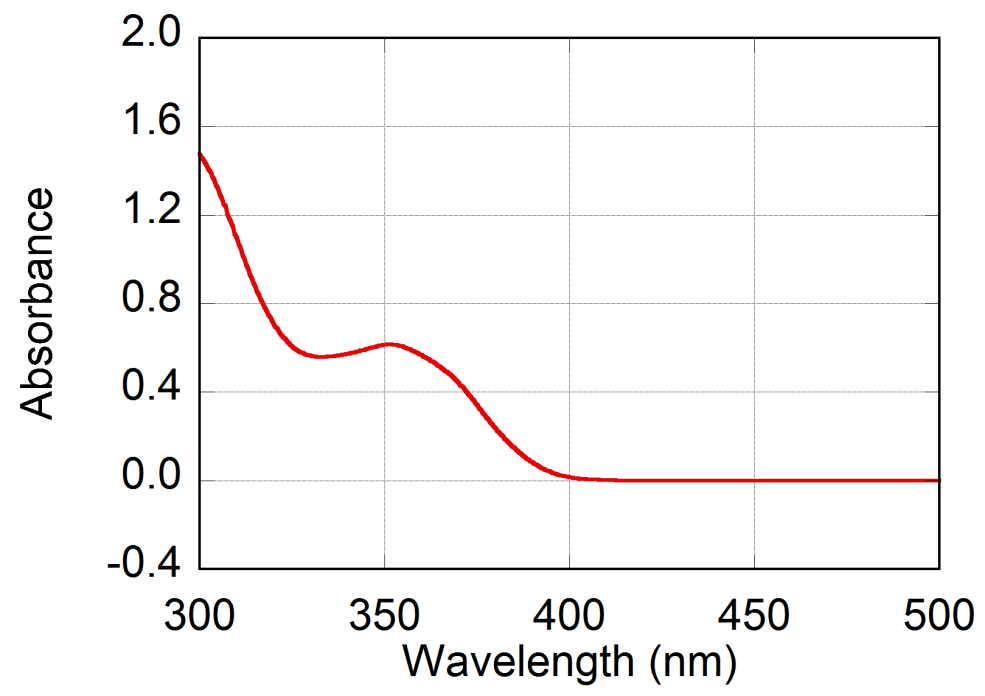

Figure S95. UV-vis absorption spectrum of 3c(5/100) in $\mathrm{PhCF}_{3}\left(3.19 \times 10^{-2} \mathrm{~g} / \mathrm{L}\right.$, light path length $=10 \mathrm{~mm}$ ).

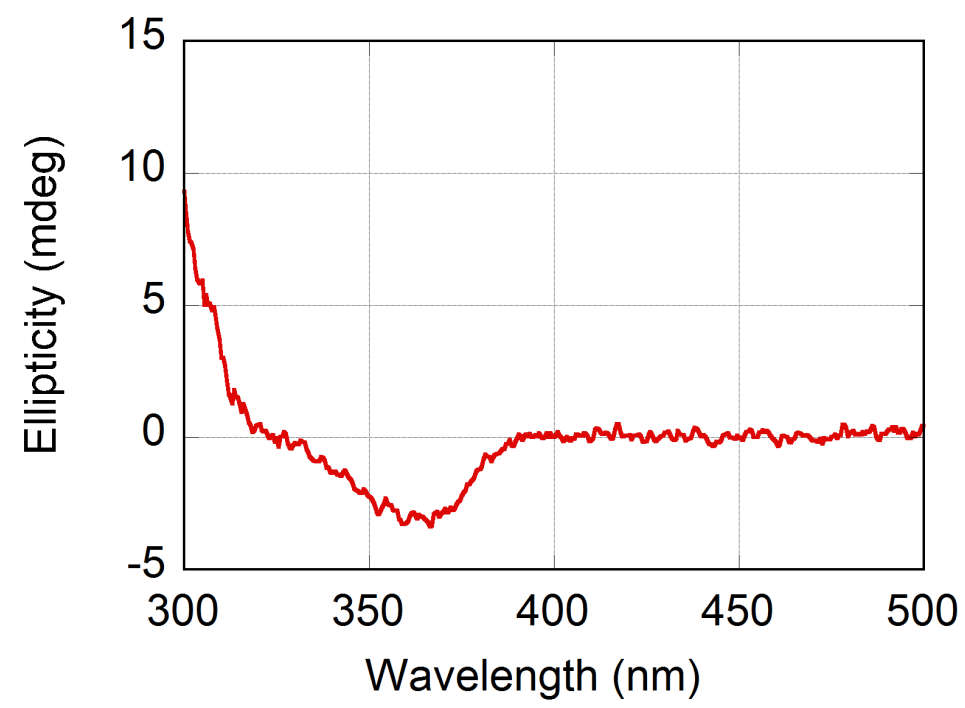

Figure S96. CD spectrum of $\mathbf{3 c}(\mathbf{5} / \mathbf{1 0 0})$ in $\mathrm{PhCF}_{3}\left(3.19 \times 10^{-2} \mathrm{~g} / \mathrm{L}\right.$, light path length $=10$ $\mathrm{mm})$. 


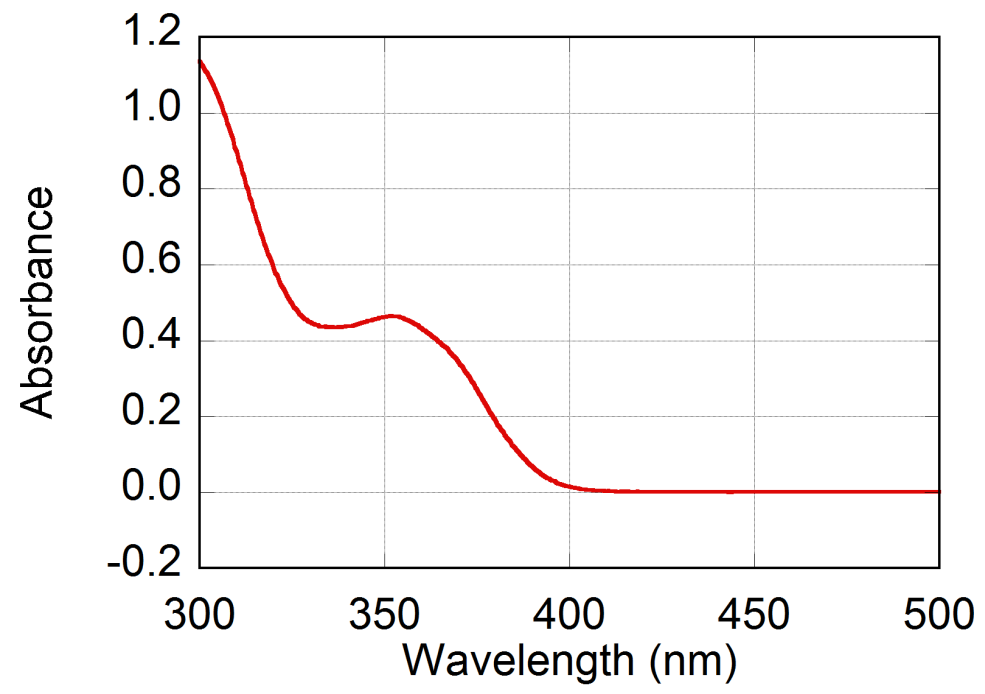

Figure S97. UV-vis absorption spectrum of 3d(5/100) in $\mathrm{PhH}\left(2.64 \times 10^{-2} \mathrm{~g} / \mathrm{L}\right.$, light path length $=10 \mathrm{~mm}$ ).

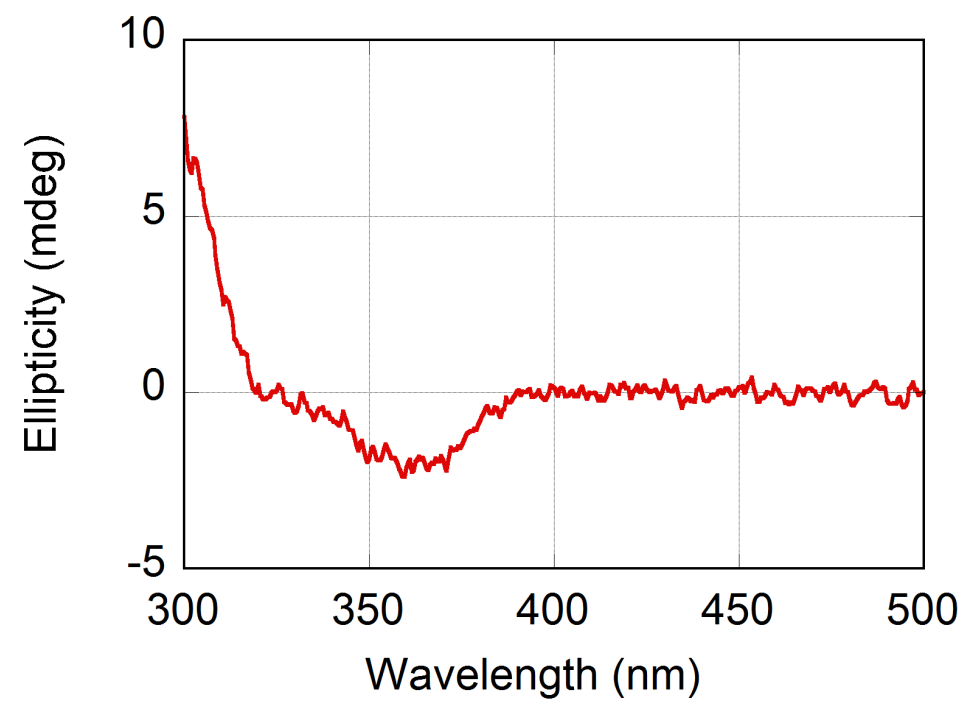

Figure S98. CD spectrum of $\mathbf{3 d}(\mathbf{5} / \mathbf{1 0 0})$ in $\mathrm{PhH}\left(2.64 \times 10^{-2} \mathrm{~g} / \mathrm{L}\right.$, light path length $=10$ $\mathrm{mm})$. 


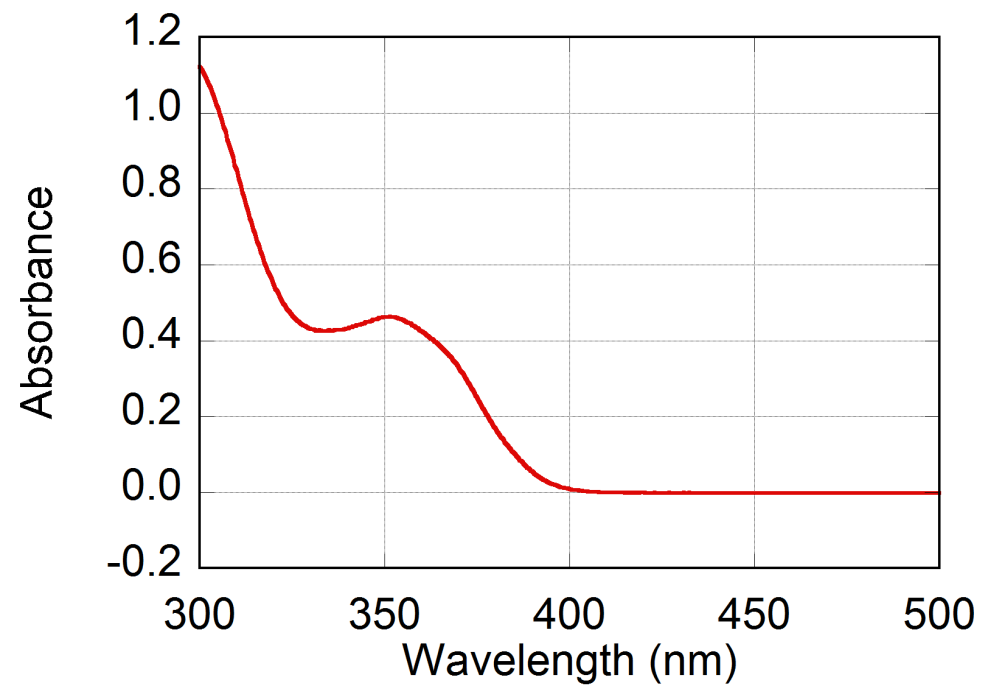

Figure S99. UV-vis absorption spectrum of 3d(5/100) in $\mathrm{PhCH}_{3}\left(2.64 \times 10^{-2} \mathrm{~g} / \mathrm{L}\right.$, light path length $=10 \mathrm{~mm}$ ).

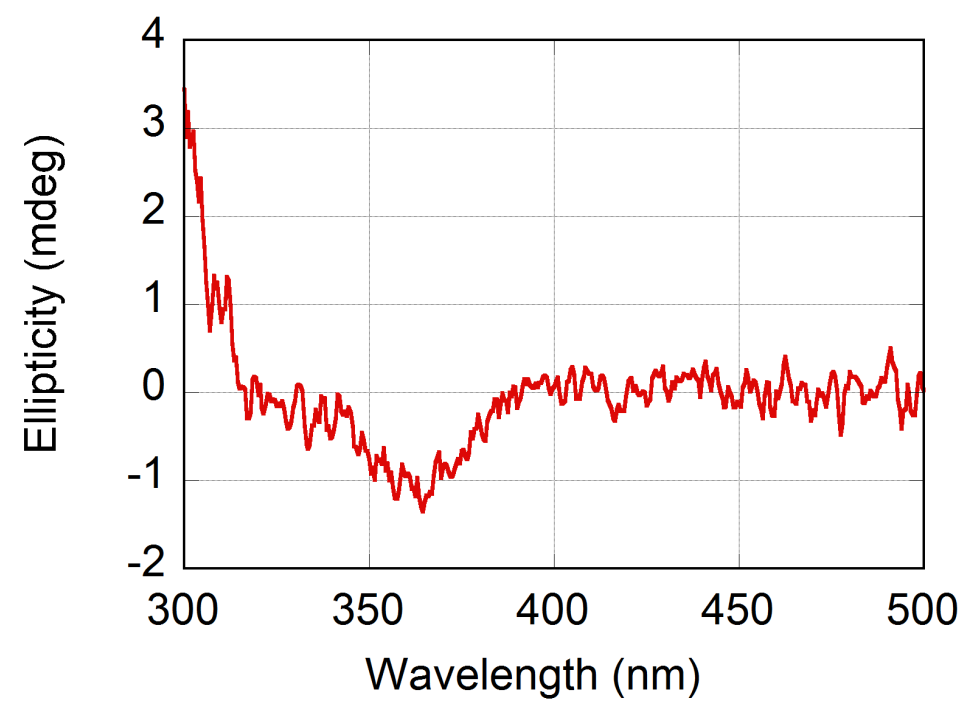

Figure S100. CD spectrum of 3d(5/100) in $\mathrm{PhCH}_{3}\left(2.64 \times 10^{-2} \mathrm{~g} / \mathrm{L}\right.$, light path length $=$ $10 \mathrm{~mm}$ ). 


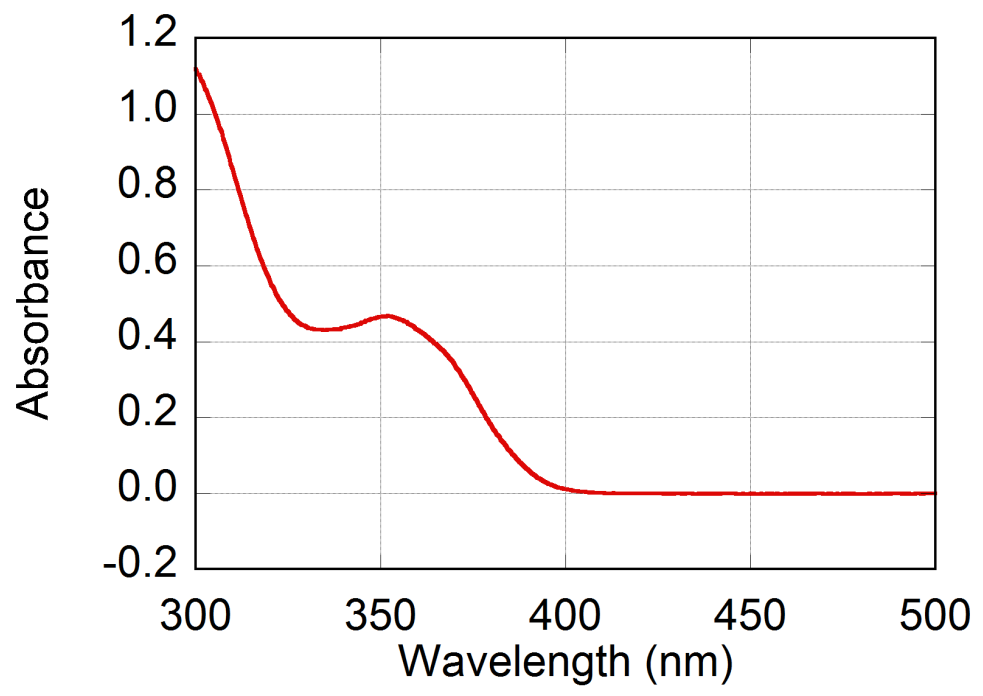

Figure S101. UV-vis absorption spectrum of 3d(5/100) in $\mathrm{PhOCH}_{3}\left(2.64 \times 10^{-2} \mathrm{~g} / \mathrm{L}\right.$, light path length $=10 \mathrm{~mm}$ ).

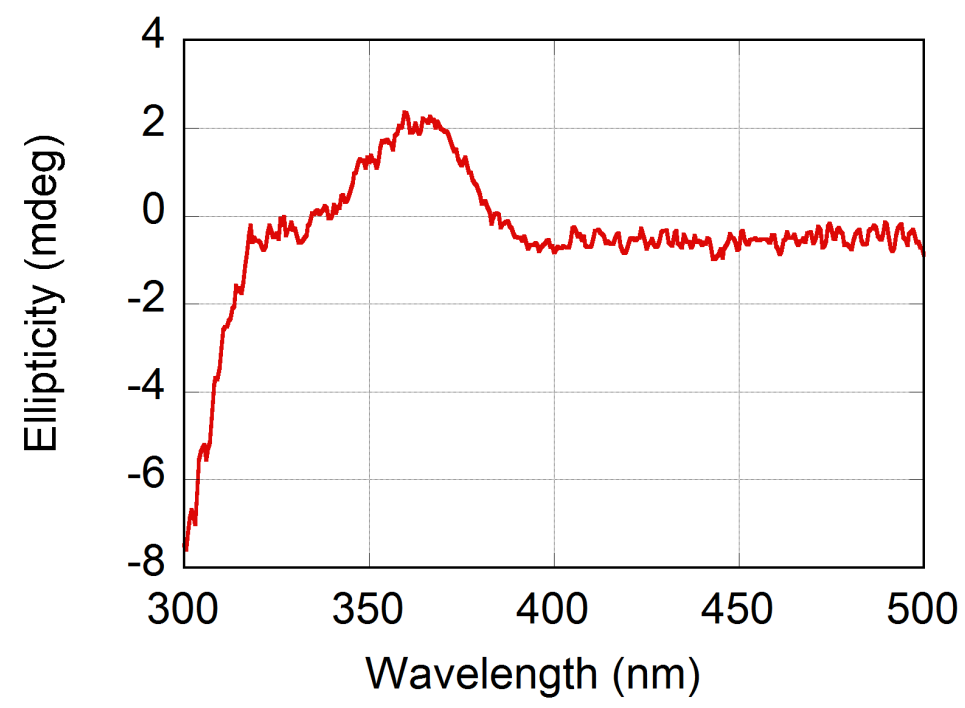

Figure S102. CD spectrum of 3d(5/100) in $\mathrm{PhOCH}_{3}\left(2.64 \times 10^{-2} \mathrm{~g} / \mathrm{L}\right.$, light path length $=10 \mathrm{~mm}$ ). 


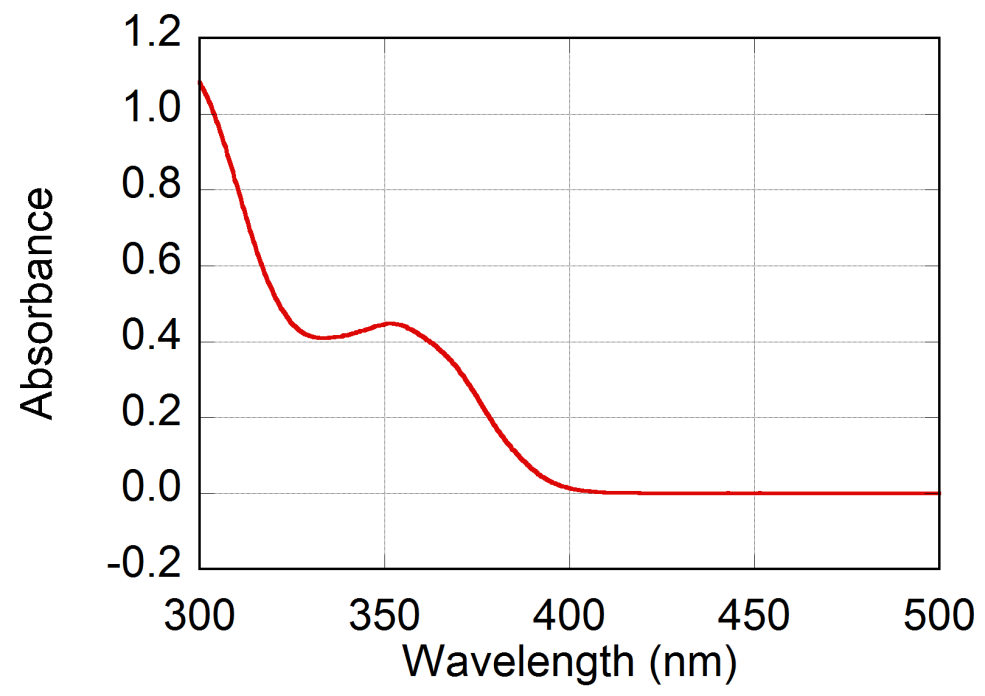

Figure S103. UV-vis absorption spectrum of 3d(5/100) in $\mathrm{PhCF}_{3}\left(2.64 \times 10^{-2} \mathrm{~g} / \mathrm{L}\right.$, light path length $=10 \mathrm{~mm}$ ).

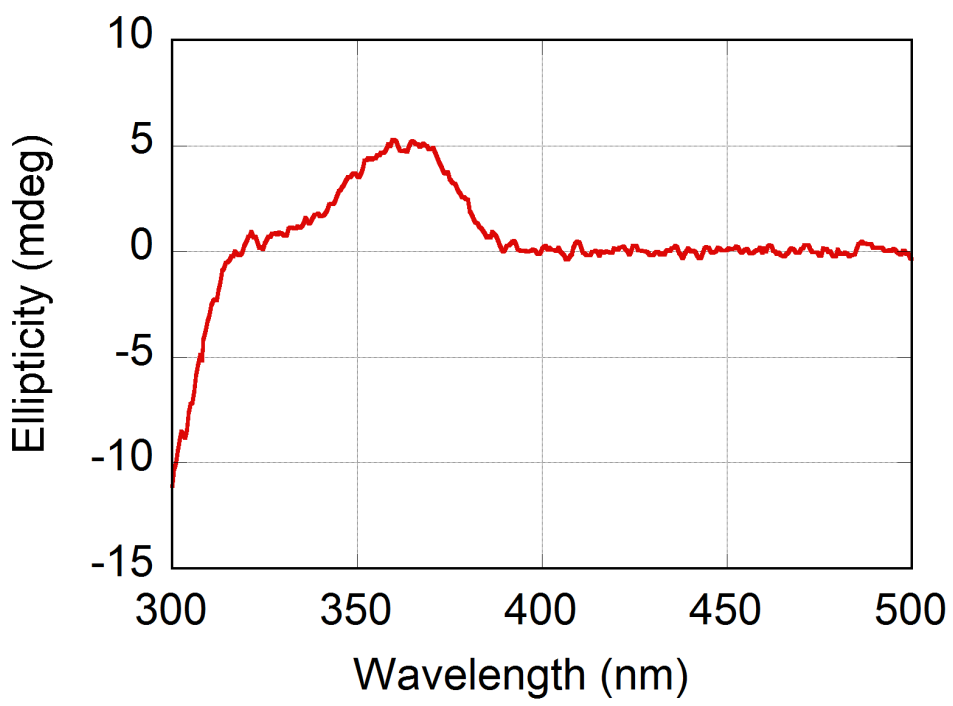

Figure S104. CD spectrum of 3d(5/100) in $\mathrm{PhCF}_{3}\left(2.64 \times 10^{-2} \mathrm{~g} / \mathrm{L}\right.$, light path length $=$ $10 \mathrm{~mm}$ ). 


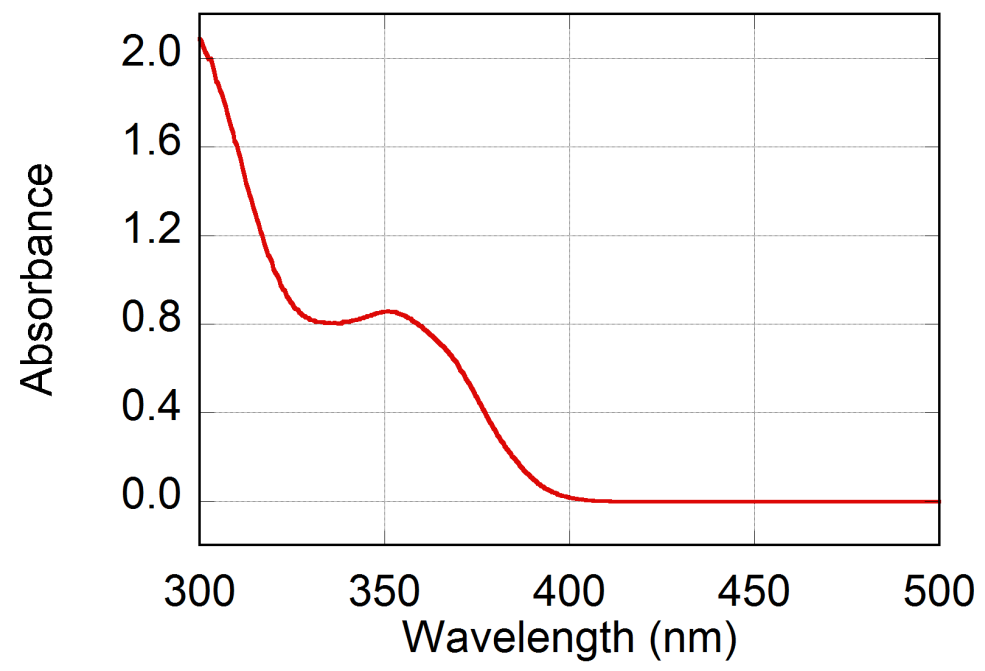

Figure S105. UV-vis absorption spectrum of $\mathbf{3 d}(\mathbf{2 . 5} / \mathbf{9 7 . 5})$ in $\mathrm{PhH}\left(2.46 \times 10^{-2} \mathrm{~g} / \mathrm{L}\right.$, light path length $=10 \mathrm{~mm}$ ).

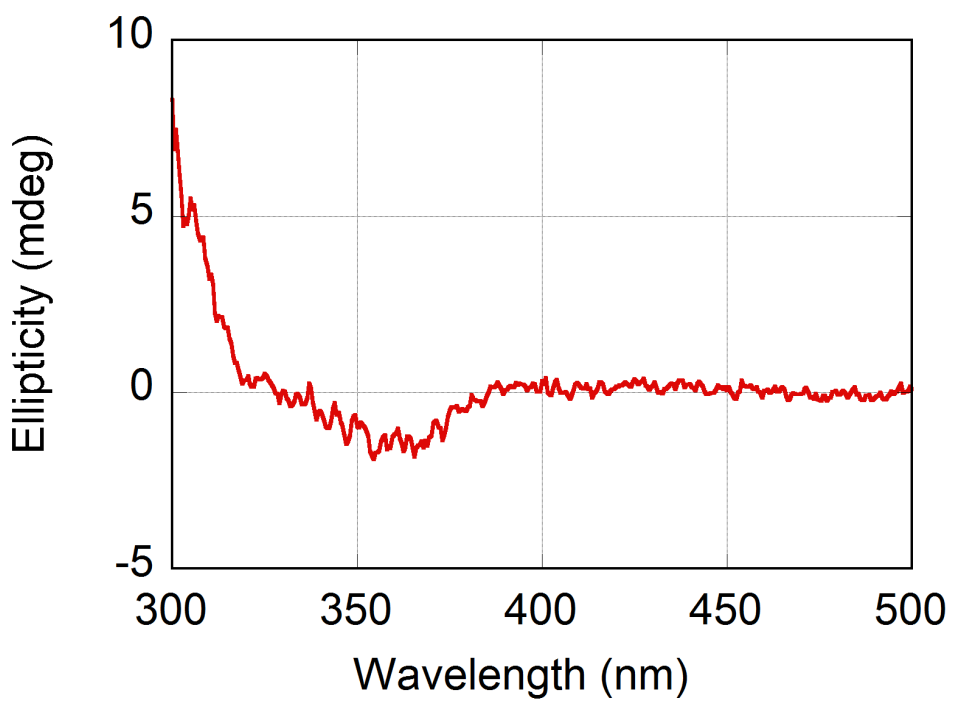

Figure S106. CD spectrum of $\mathbf{3 d} \mathbf{d}(\mathbf{2 . 5} / \mathbf{9 7 . 5})$ in $\mathrm{PhH}\left(2.46 \times 10^{-2} \mathrm{~g} / \mathrm{L}\right.$, light path length $=$ $10 \mathrm{~mm}$ ). 


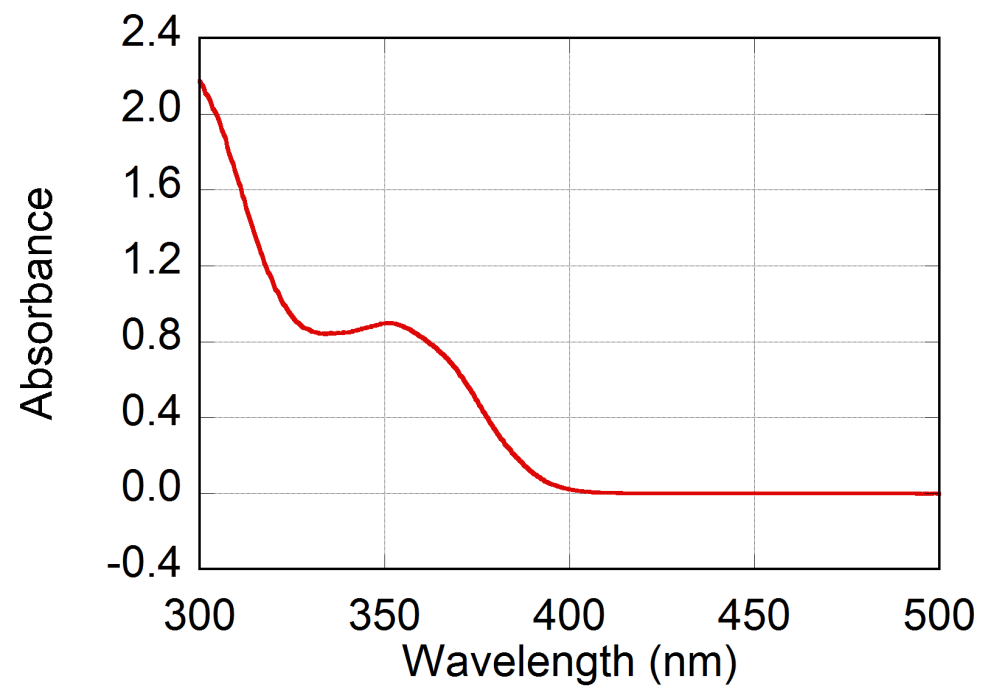

Figure S107. UV-vis absorption spectrum of 3d(5/95) in $\mathrm{PhH}\left(2.55 \times 10^{-2} \mathrm{~g} / \mathrm{L}\right.$, light path length $=10 \mathrm{~mm}$ ).

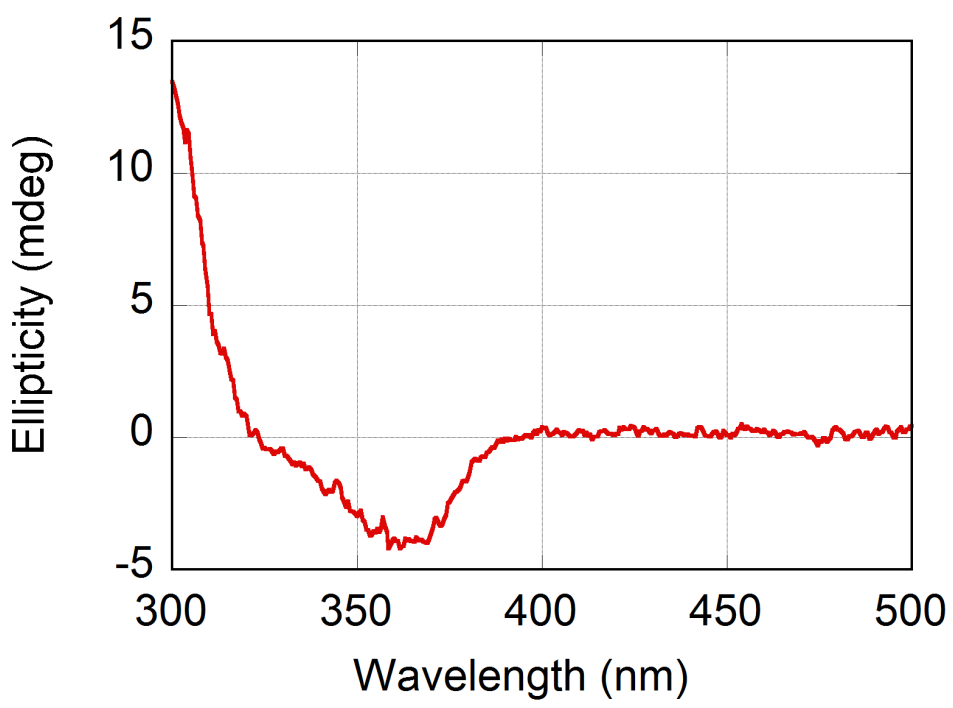

Figure S108. CD spectrum of $\mathbf{3 d}(\mathbf{5} / \mathbf{9 5})$ in $\mathrm{PhH}\left(2.55 \times 10^{-2} \mathrm{~g} / \mathrm{L}\right.$, light path length $=10$ $\mathrm{mm})$. 


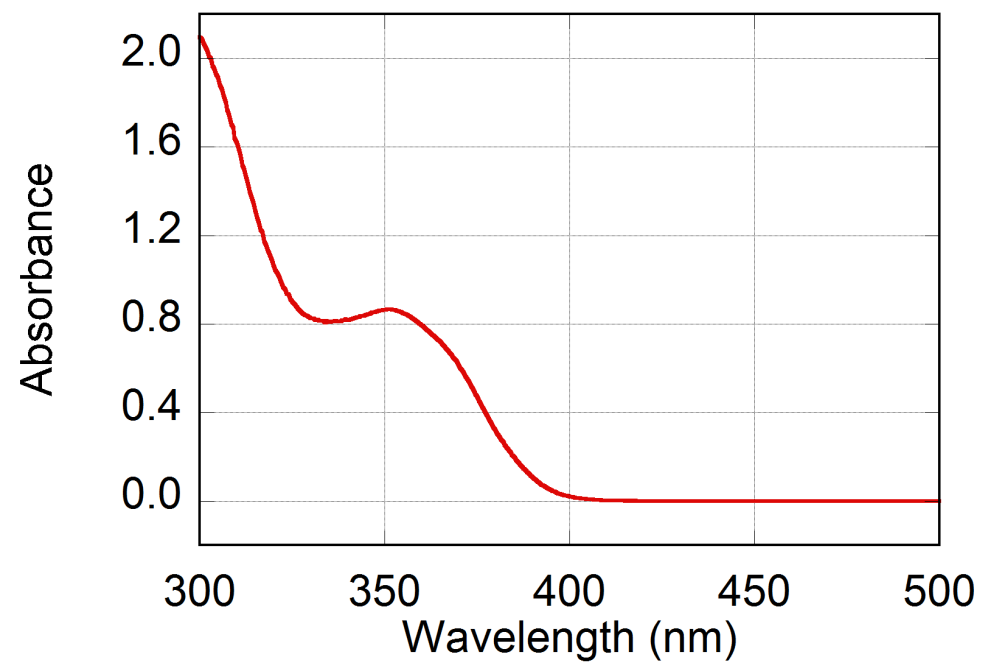

Figure S109. UV-vis absorption spectrum of 3d(7.5/92.5) in $\mathrm{PhH}\left(2.61 \times 10^{-2} \mathrm{~g} / \mathrm{L}\right.$, light path length $=10 \mathrm{~mm}$ ).

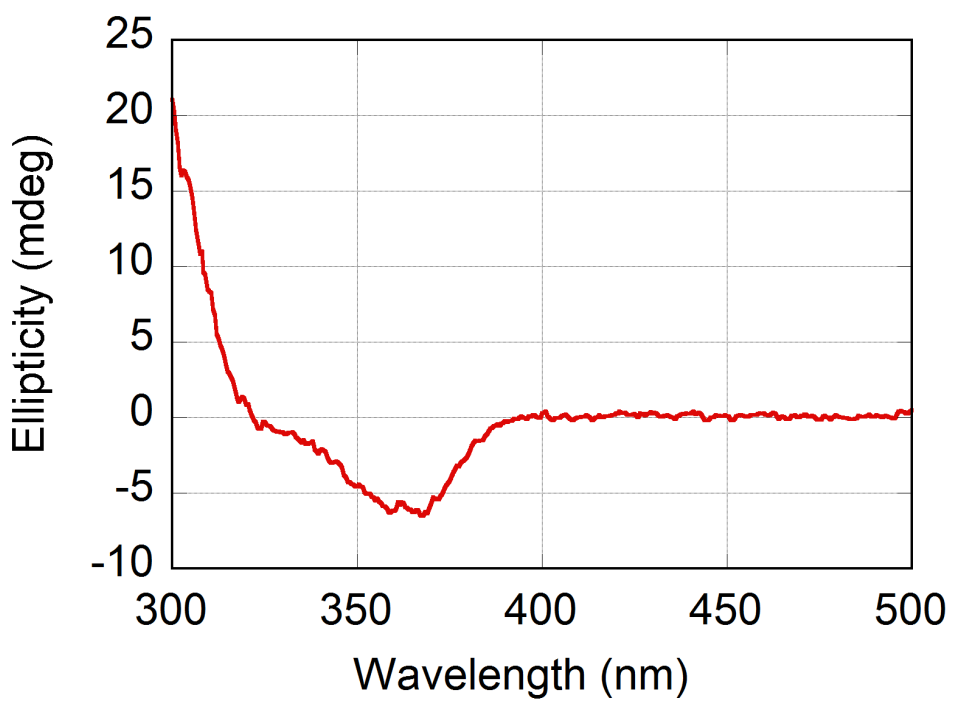

Figure S110. CD spectrum of 3d(7.5/92.5) in $\mathrm{PhH}\left(2.61 \times 10^{-2} \mathrm{~g} / \mathrm{L}\right.$, light path length $=$ $10 \mathrm{~mm}$ ). 


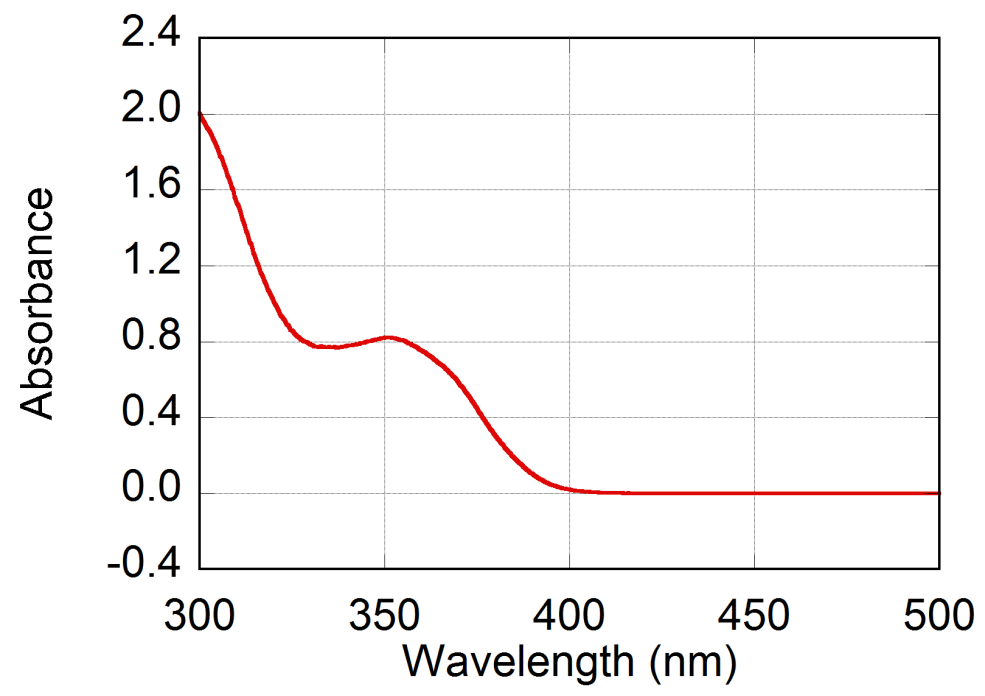

Figure S111. UV-vis absorption spectrum of 3d(10/90) in $\mathrm{PhH}\left(2.55 \times 10^{-2} \mathrm{~g} / \mathrm{L}\right.$, light path length $=10 \mathrm{~mm}$ ).

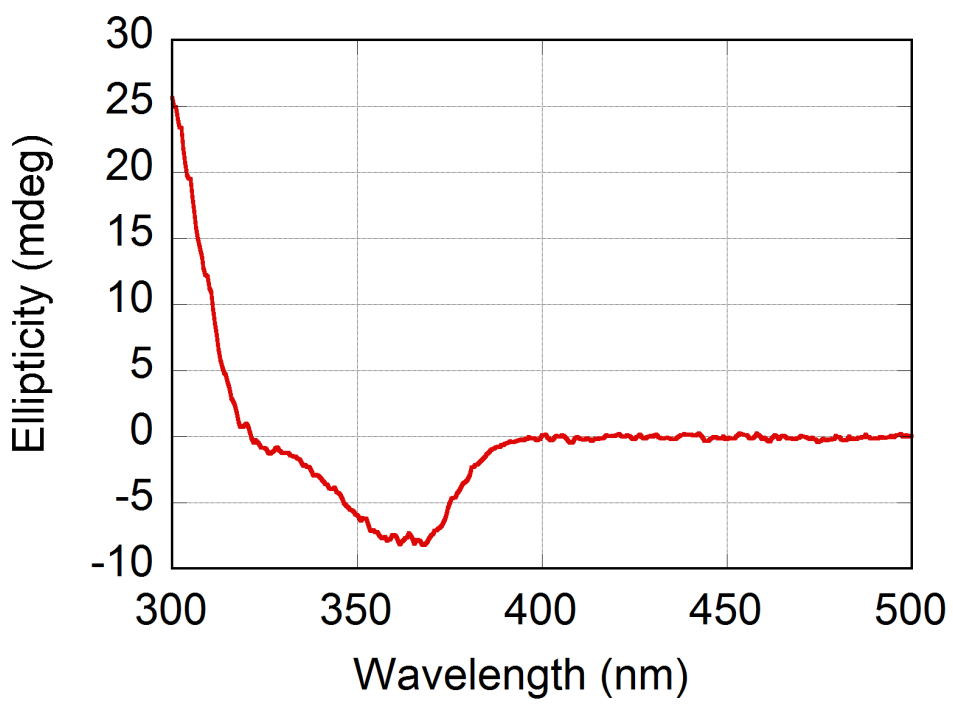

Figure S112. CD spectrum of $\mathbf{3 d}(\mathbf{1 0} / \mathbf{9 0})$ in $\mathrm{PhH}\left(2.55 \times 10^{-2} \mathrm{~g} / \mathrm{L}\right.$, light path length $=10$ $\mathrm{mm})$. 


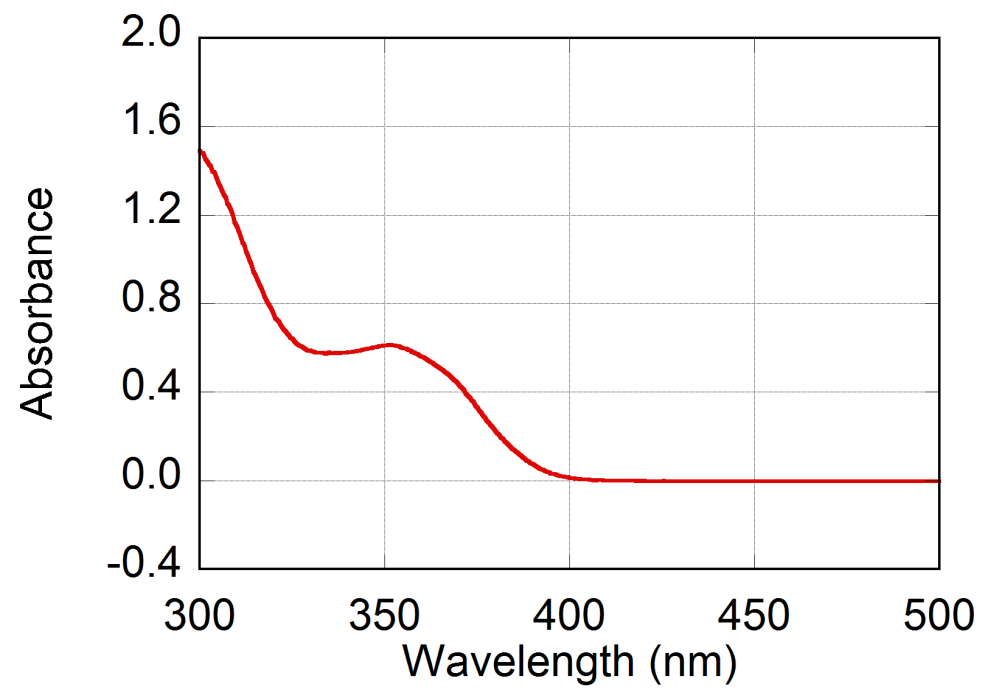

Figure S113. UV-vis absorption spectrum of $\mathbf{3 d}(\mathbf{1 5} / \mathbf{8 5})$ in $\mathrm{PhH}\left(1.88 \times 10^{-2} \mathrm{~g} / \mathrm{L}\right.$, light path length $=10 \mathrm{~mm}$ ).

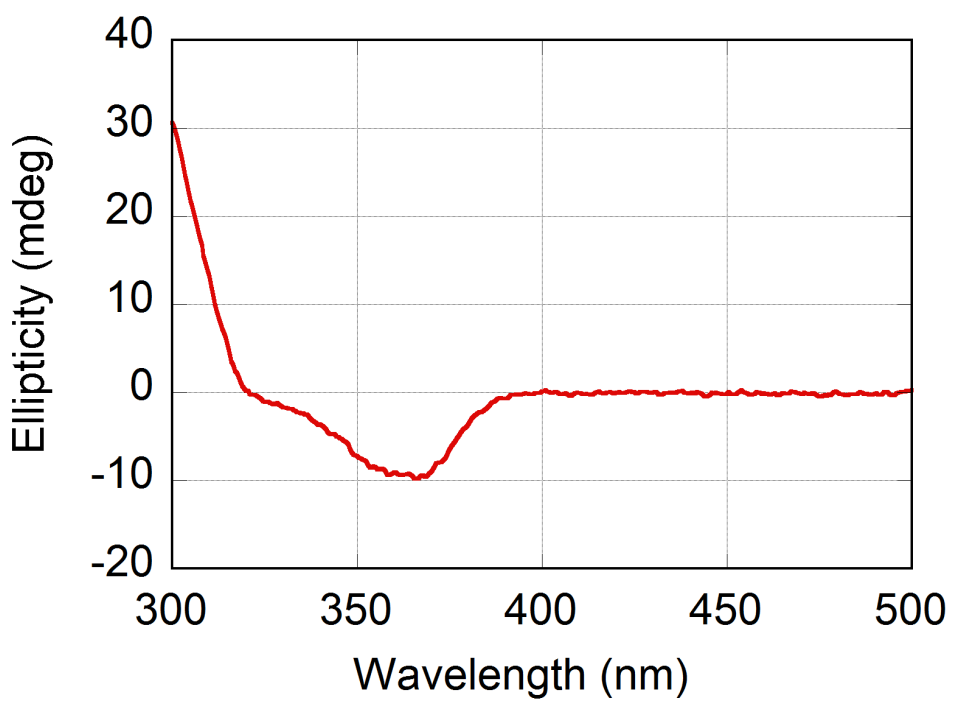

Figure S114. CD spectrum of $\mathbf{3 d}(\mathbf{1 5} / \mathbf{8 5})$ in $\mathrm{PhH}\left(1.88 \times 10^{-2} \mathrm{~g} / \mathrm{L}\right.$, light path length $=10$ $\mathrm{mm})$. 


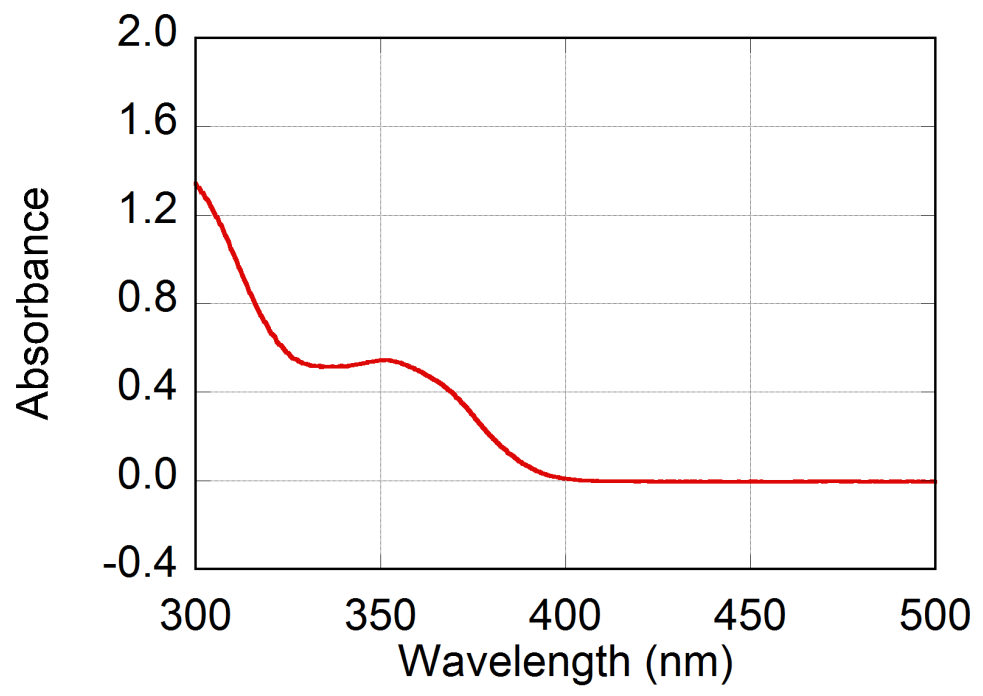

Figure S115. UV-vis absorption spectrum of 3d(20/80) in $\mathrm{PhH}\left(1.22 \times 10^{-2} \mathrm{~g} / \mathrm{L}\right.$, light path length $=10 \mathrm{~mm}$ ).

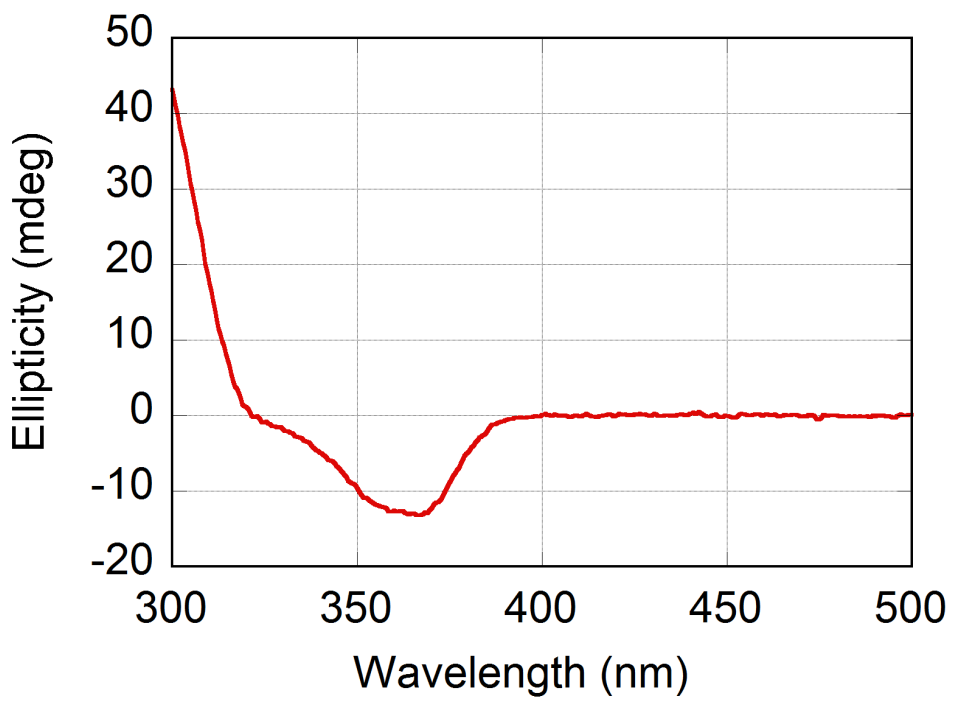

Figure S116. CD spectrum of $\mathbf{3 d}(\mathbf{2 0} / \mathbf{8 0})$ in $\mathrm{PhH}\left(1.22 \times 10^{-2} \mathrm{~g} / \mathrm{L}\right.$, light path length $=10$ $\mathrm{mm})$. 


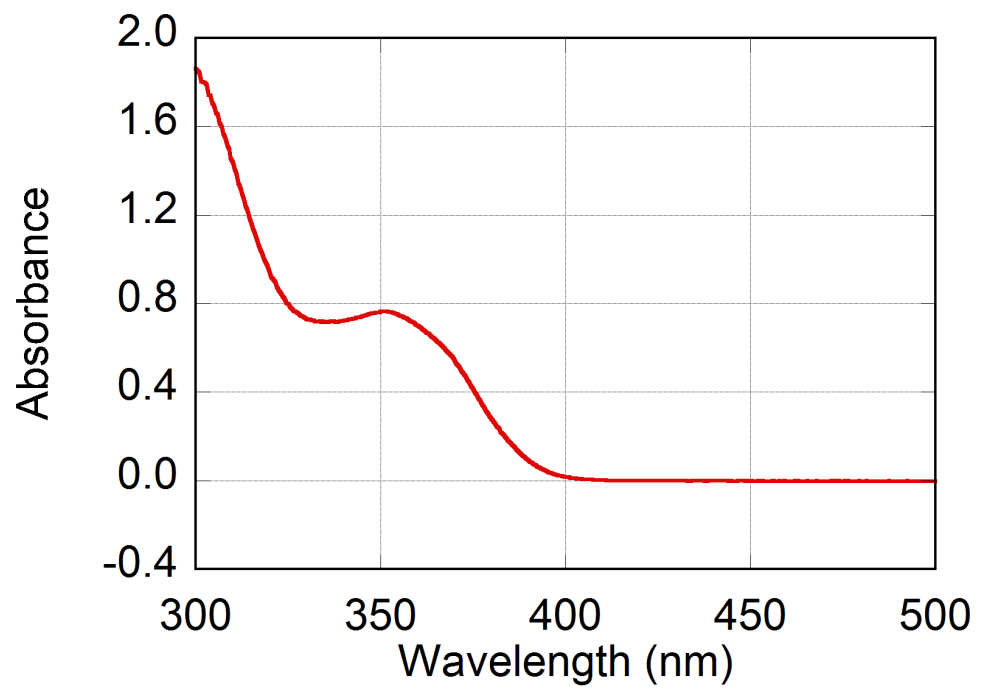

Figure S117. UV-vis absorption spectrum of 3d(30/70) in $\mathrm{PhH}\left(2.60 \times 10^{-2} \mathrm{~g} / \mathrm{L}\right.$, light path length $=10 \mathrm{~mm}$ ).

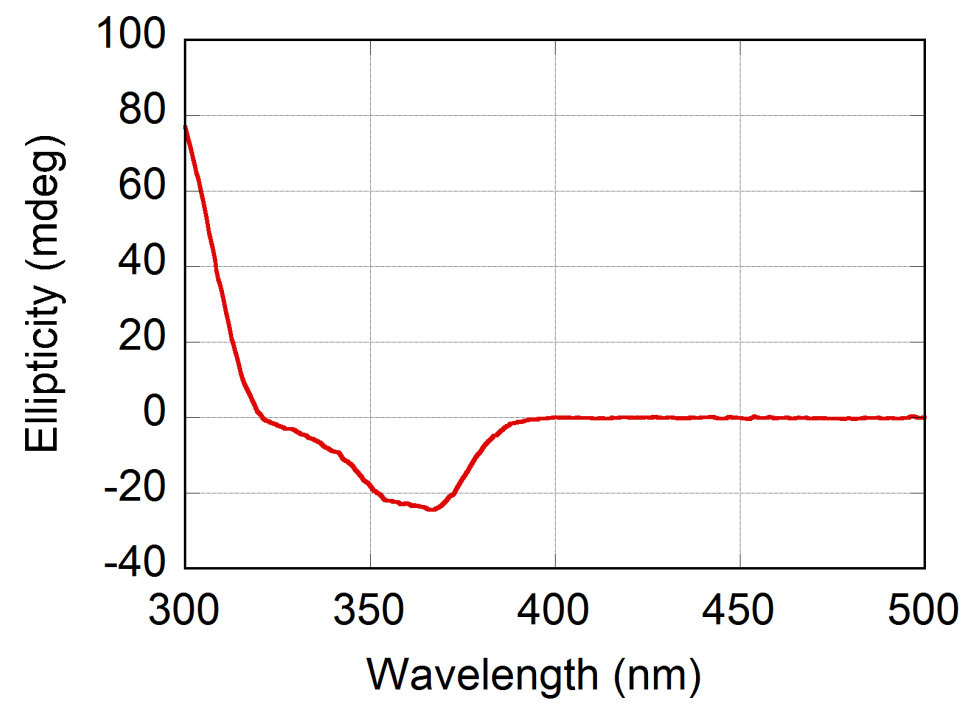

Figure S118. CD spectrum of $\mathbf{3 d}(\mathbf{3 0} / \mathbf{7 0})$ in $\mathrm{PhH}\left(2.60 \times 10^{-2} \mathrm{~g} / \mathrm{L}\right.$, light path length $=10$ $\mathrm{mm})$. 


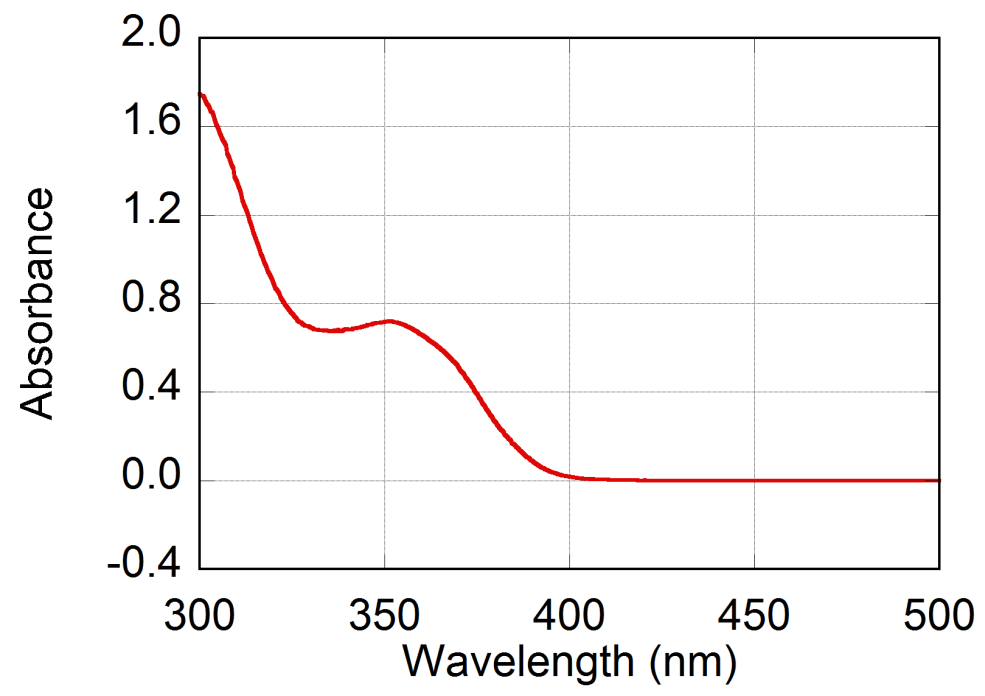

Figure S119. UV-vis absorption spectrum of 3d(40/60) in $\mathrm{PhH}\left(2.45 \times 10^{-2} \mathrm{~g} / \mathrm{L}\right.$, light path length $=10 \mathrm{~mm}$ ).

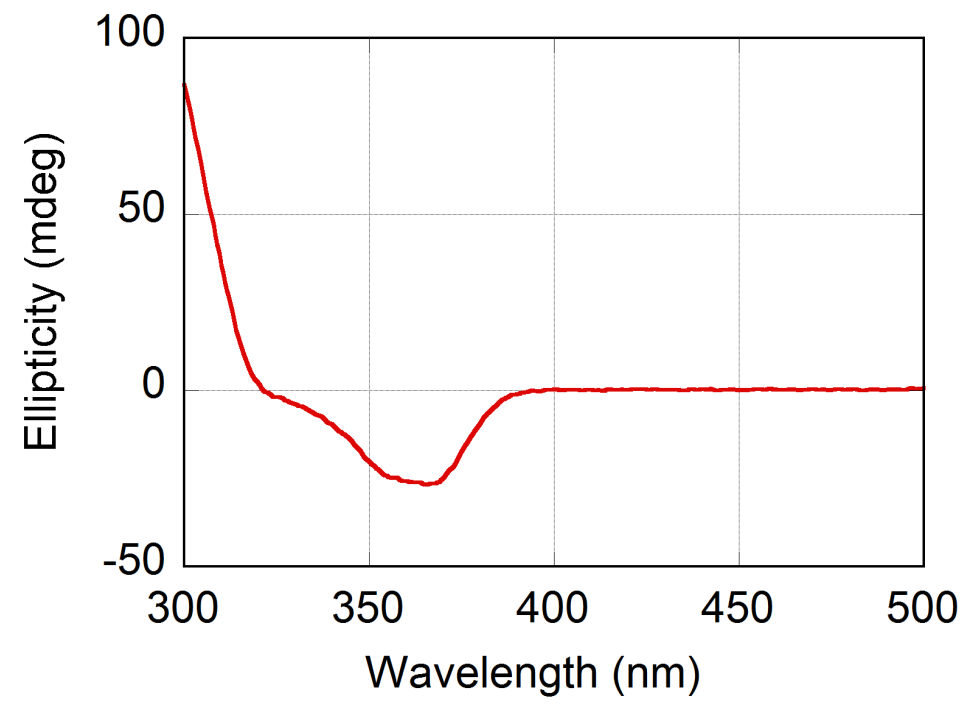

Figure S120. CD spectrum of $\mathbf{3 d}(\mathbf{4 0} / \mathbf{6 0})$ in $\mathrm{PhH}\left(2.45 \times 10^{-2} \mathrm{~g} / \mathrm{L}\right.$, light path length $=10$ $\mathrm{mm})$. 


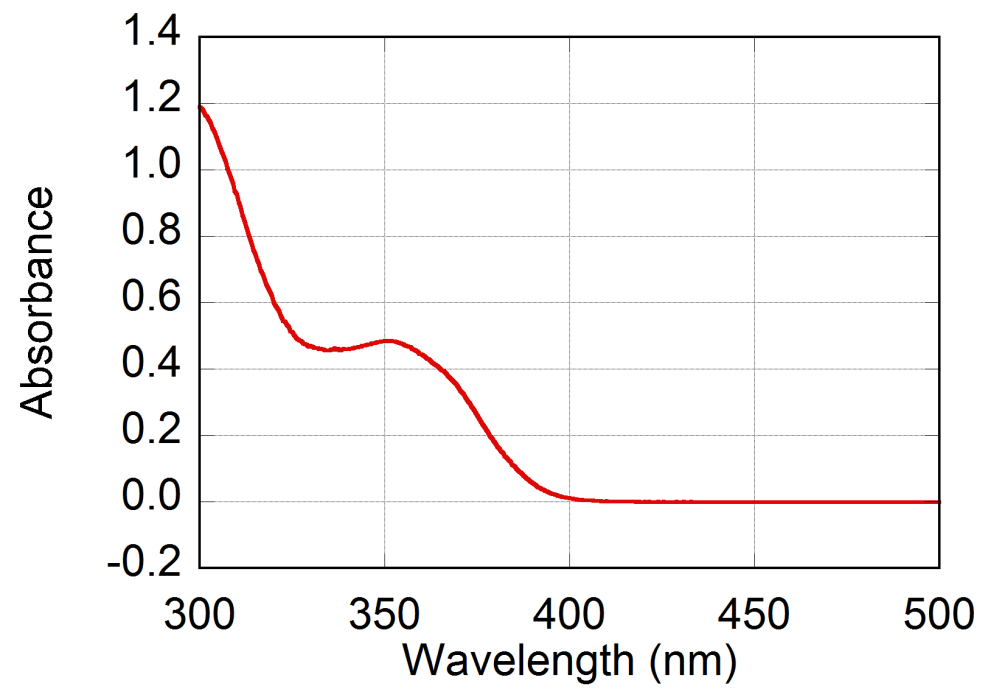

Figure S121. UV-vis absorption spectrum of $\mathbf{3 d}(\mathbf{6 0} / \mathbf{4 0})$ in $\mathrm{PhH}\left(2.15 \times 10^{-2} \mathrm{~g} / \mathrm{L}\right.$, light path length $=10 \mathrm{~mm}$ ).

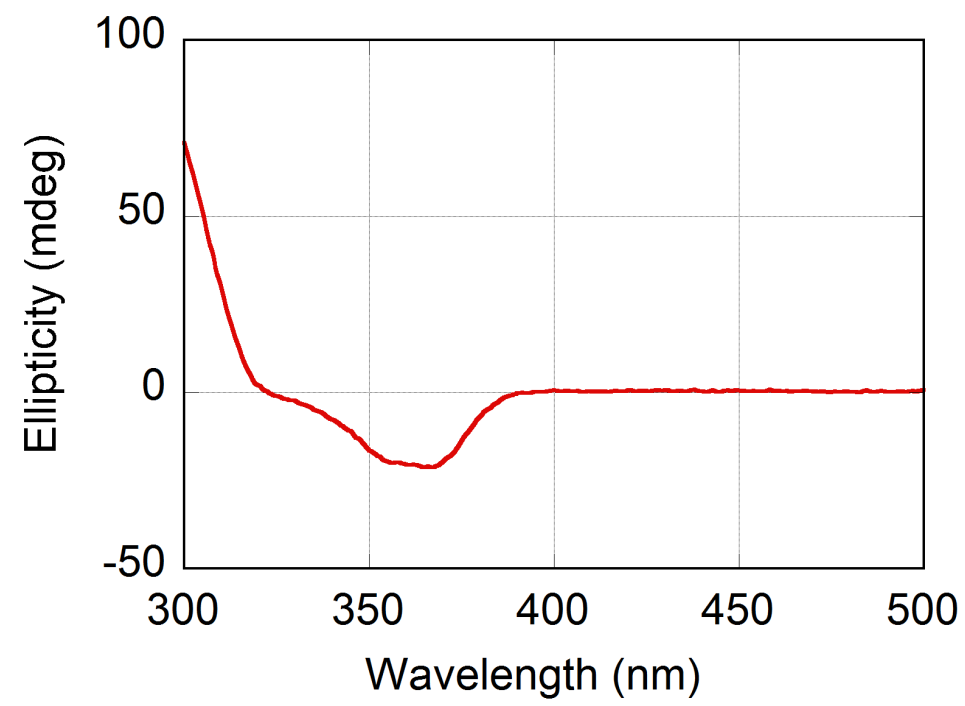

Figure S122. CD spectrum of $\mathbf{3 d}(\mathbf{6 0} / \mathbf{4 0})$ in $\mathrm{PhH}\left(2.15 \times 10^{-2} \mathrm{~g} / \mathrm{L}\right.$, light path length $=10$ $\mathrm{mm})$. 


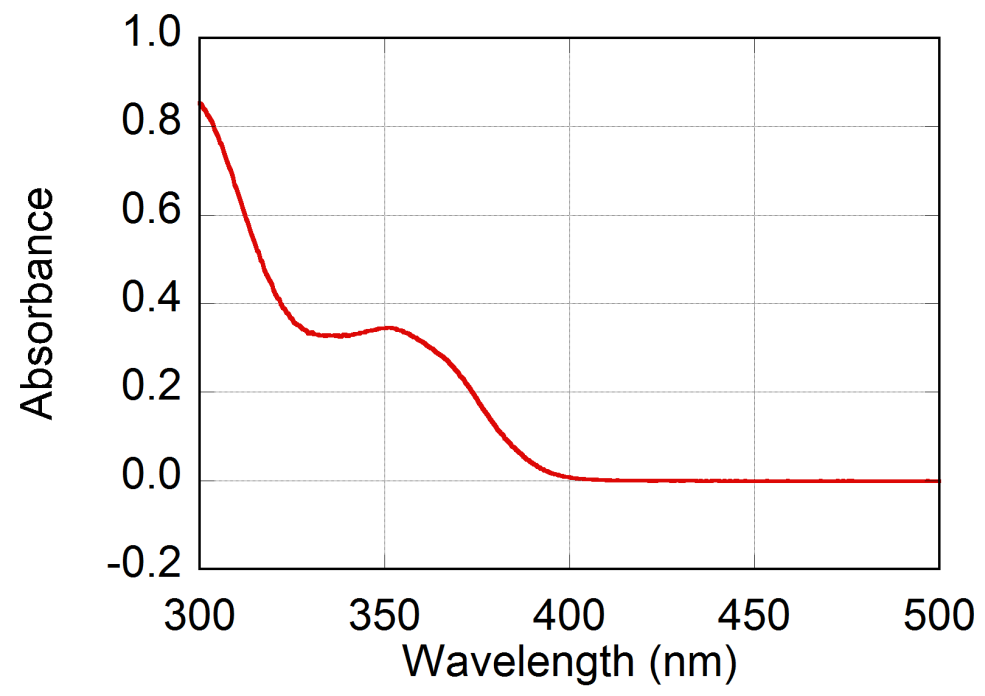

Figure S123. UV-vis absorption spectrum of $\mathbf{3 d}(\mathbf{8 0} / \mathbf{2 0})$ in $\mathrm{PhH}\left(1.75 \times 10^{-2} \mathrm{~g} / \mathrm{L}\right.$, light path length $=10 \mathrm{~mm}$ ).

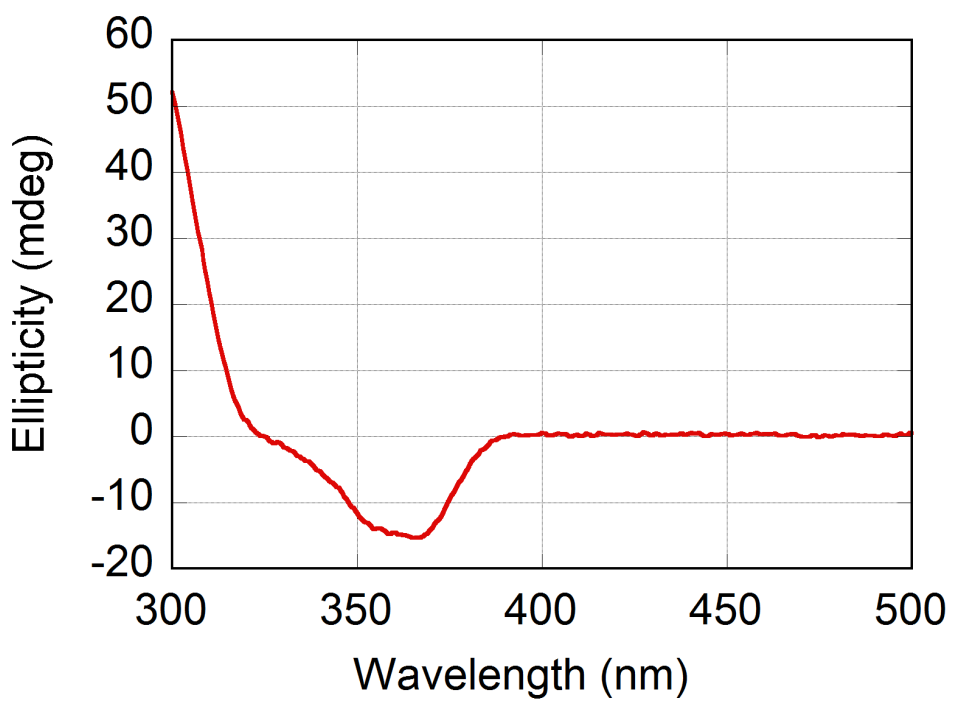

Figure S124. CD spectrum of $\mathbf{3 d}(\mathbf{8 0} / \mathbf{2 0})$ in $\mathrm{PhH}\left(1.75 \times 10^{-2} \mathrm{~g} / \mathrm{L}\right.$, light path length $=10$ $\mathrm{mm})$. 


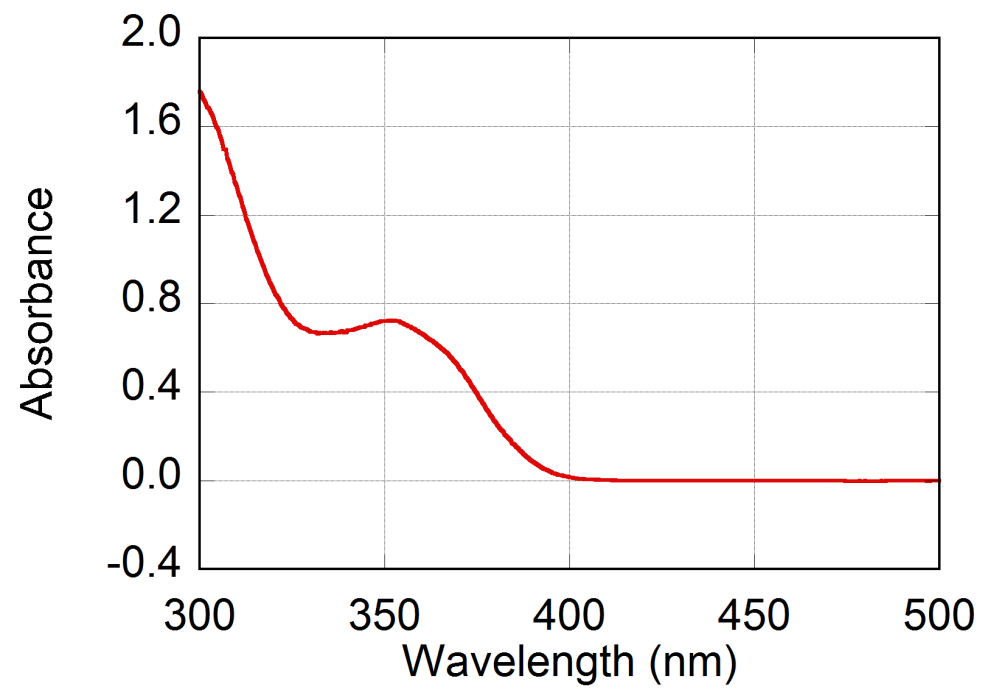

Figure S125. UV-vis absorption spectrum of 3d(2.5/97.5) in $\mathrm{PhCH}_{3}\left(2.46 \times 10^{-2} \mathrm{~g} / \mathrm{L}\right.$, light path length $=10 \mathrm{~mm}$ ).

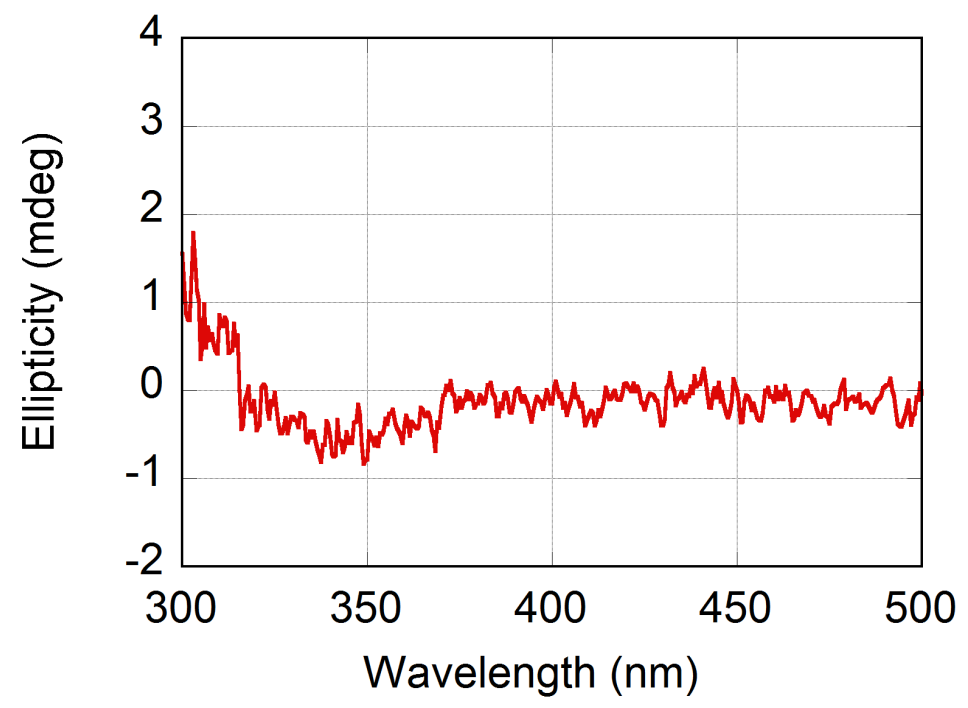

Figure S126. CD spectrum of $\mathbf{3 d}(\mathbf{2 . 5} / \mathbf{9 7 . 5})$ in $\mathrm{PhCH}_{3}\left(2.46 \times 10^{-2} \mathrm{~g} / \mathrm{L}\right.$, light path length $=10 \mathrm{~mm}$ ). 


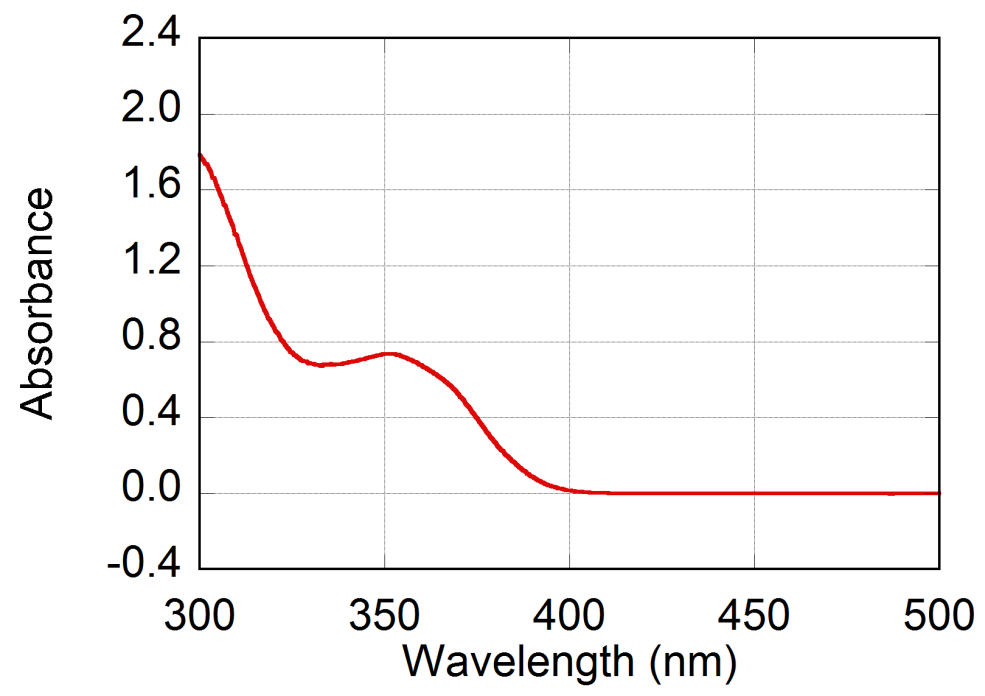

Figure S127. UV-vis absorption spectrum of 3d(5/95) in $\mathrm{PhCH}_{3}\left(2.55 \times 10^{-2} \mathrm{~g} / \mathrm{L}\right.$, light path length $=10 \mathrm{~mm}$ ).

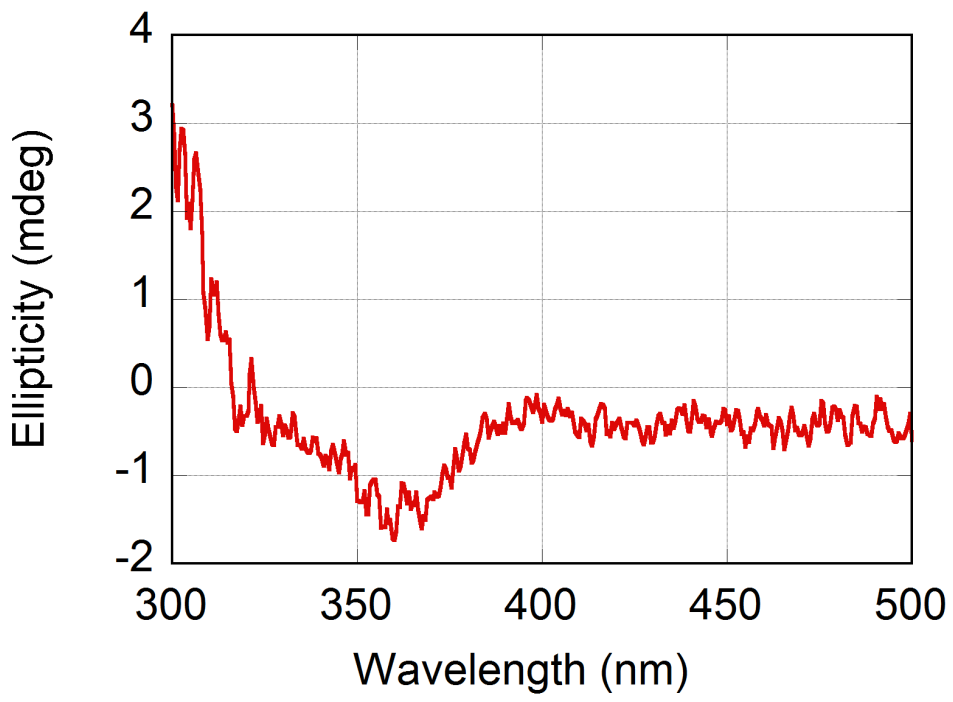

Figure S128. CD spectrum of $\mathbf{3 d}(\mathbf{5} / \mathbf{9 5})$ in $\mathrm{PhCH}_{3}\left(2.55 \times 10^{-2} \mathrm{~g} / \mathrm{L}\right.$, light path length $=$ $10 \mathrm{~mm}$ ). 


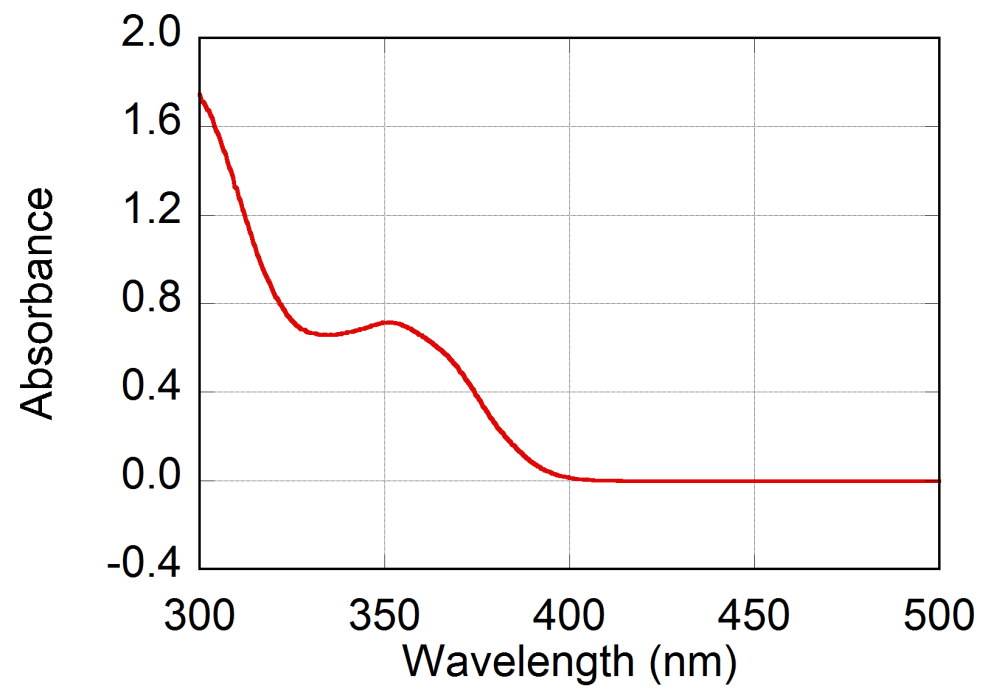

Figure S129. UV-vis absorption spectrum of 3d(7.5/92.5) in $\mathrm{PhCH}_{3}\left(2.61 \times 10^{-2} \mathrm{~g} / \mathrm{L}\right.$, light path length $=10 \mathrm{~mm}$ ).

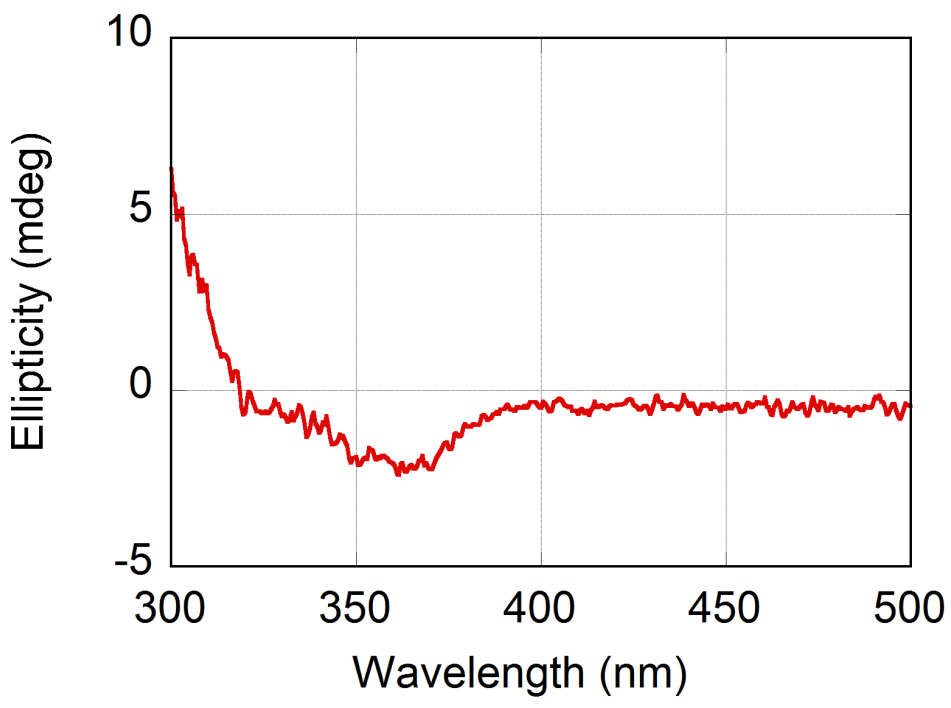

Figure S130. CD spectrum of $\mathbf{3 d} \mathbf{d}(\mathbf{7 . 5} / \mathbf{9 2} .5)$ in $\mathrm{PhCH}_{3}\left(2.61 \times 10^{-2} \mathrm{~g} / \mathrm{L}\right.$, light path length $=10 \mathrm{~mm}$ ). 


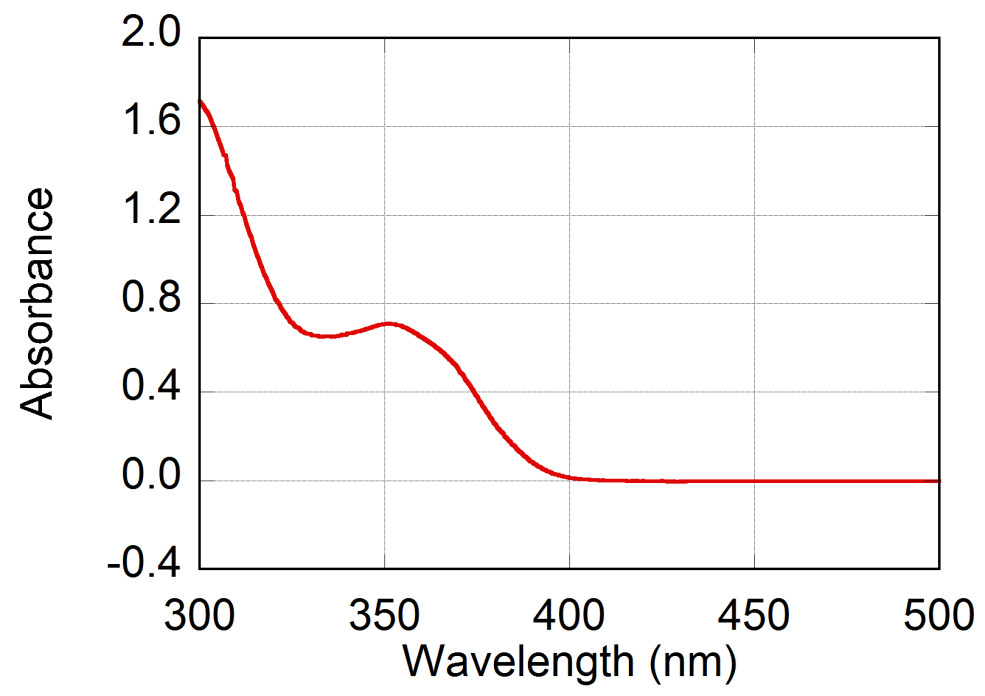

Figure S131. UV-vis absorption spectrum of 3d(10/90) in $\mathrm{PhCH}_{3}\left(2.55 \times 10^{-2} \mathrm{~g} / \mathrm{L}\right.$, light path length $=10 \mathrm{~mm}$ ).

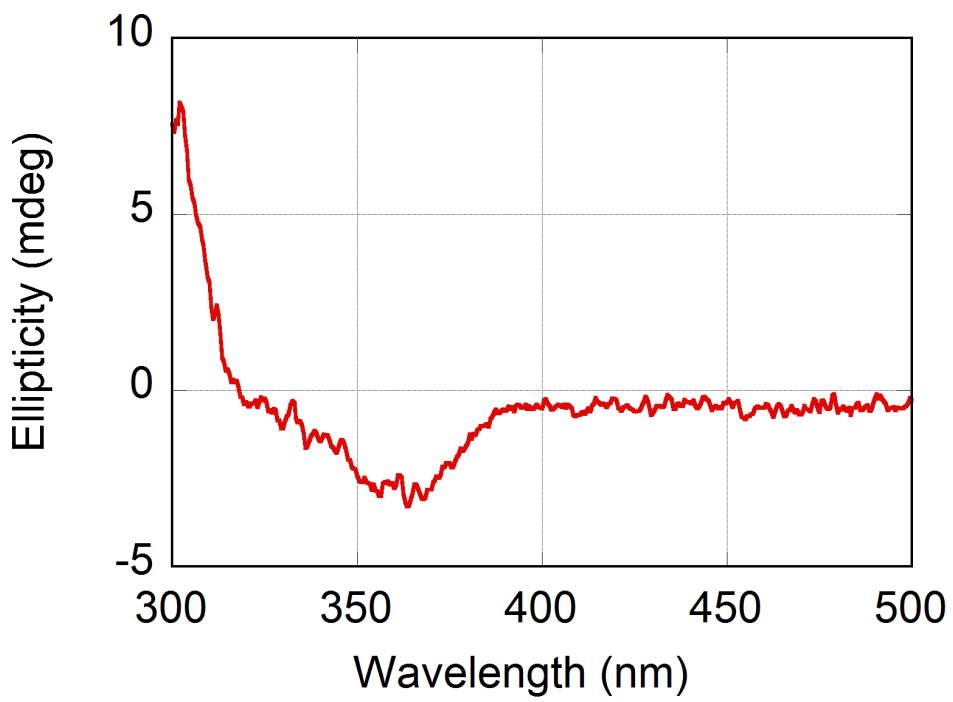

Figure S132. CD spectrum of 3d(10/90) in $\mathrm{PhCH}_{3}\left(2.55 \times 10^{-2} \mathrm{~g} / \mathrm{L}\right.$, light path length $=$ $10 \mathrm{~mm}$ ). 


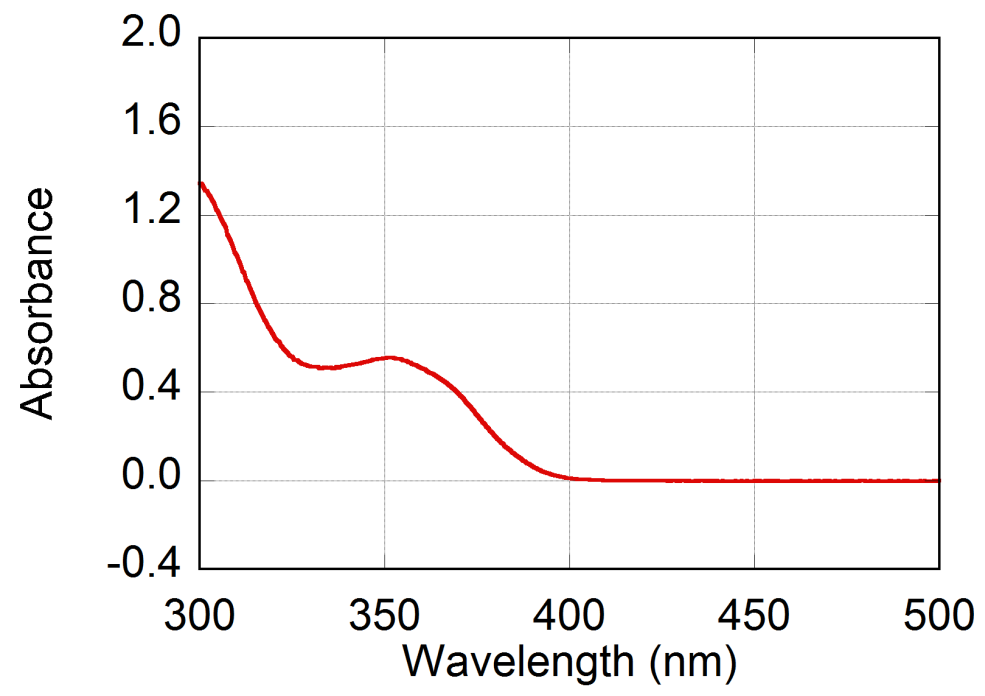

Figure S133. UV-vis absorption spectrum of $\mathbf{3 d}(\mathbf{1 5} / \mathbf{8 5})$ in $\mathrm{PhCH}_{3}\left(1.88 \times 10^{-2} \mathrm{~g} / \mathrm{L}\right.$, light path length $=10 \mathrm{~mm}$ ).

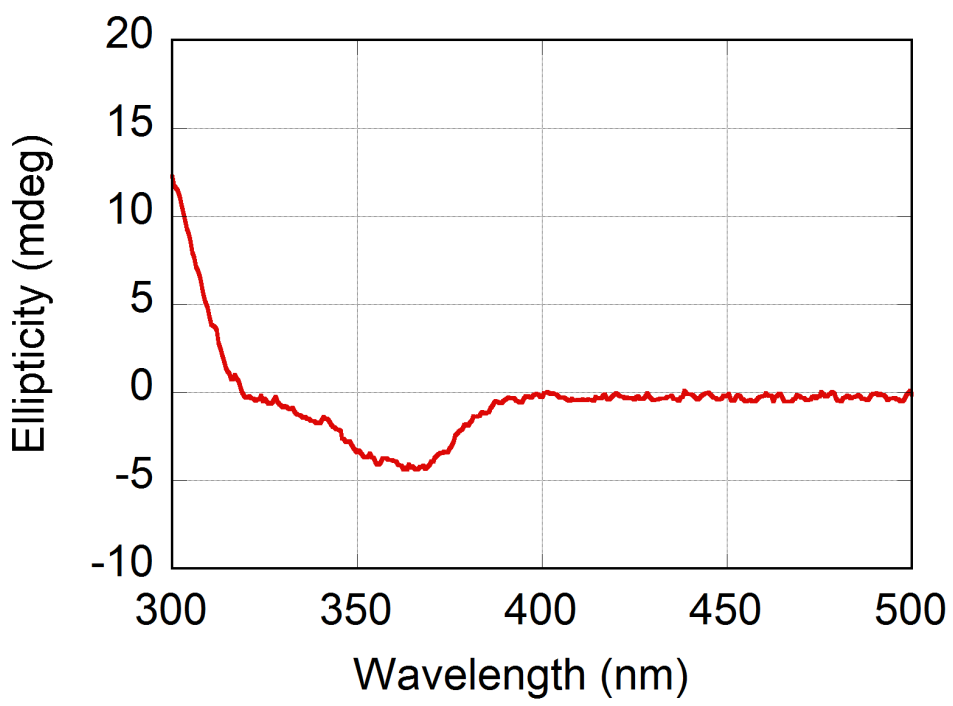

Figure S134. CD spectrum of $\mathbf{3 d}(\mathbf{1 5} / \mathbf{8 5})$ in $\mathrm{PhCH}_{3}\left(1.88 \times 10^{-2} \mathrm{~g} / \mathrm{L}\right.$, light path length $=$ $10 \mathrm{~mm}$ ). 


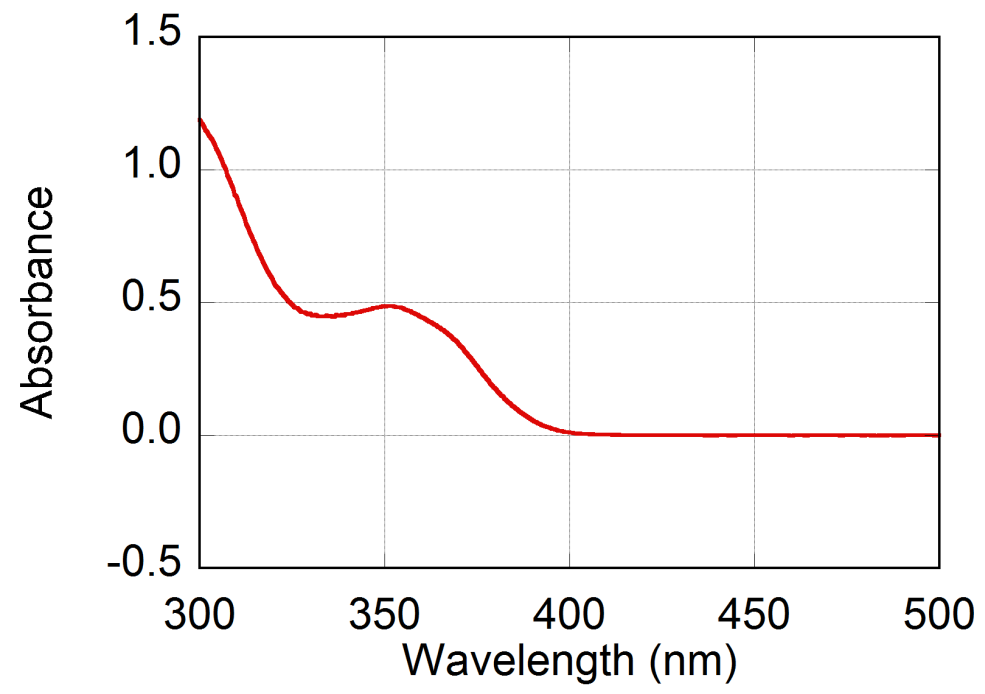

Figure S135. UV-vis absorption spectrum of $\mathbf{3 d}(\mathbf{2 0} / \mathbf{8 0})$ in $\mathrm{PhCH}_{3}\left(1.22 \times 10^{-2} \mathrm{~g} / \mathrm{L}\right.$, light path length $=10 \mathrm{~mm}$ ).

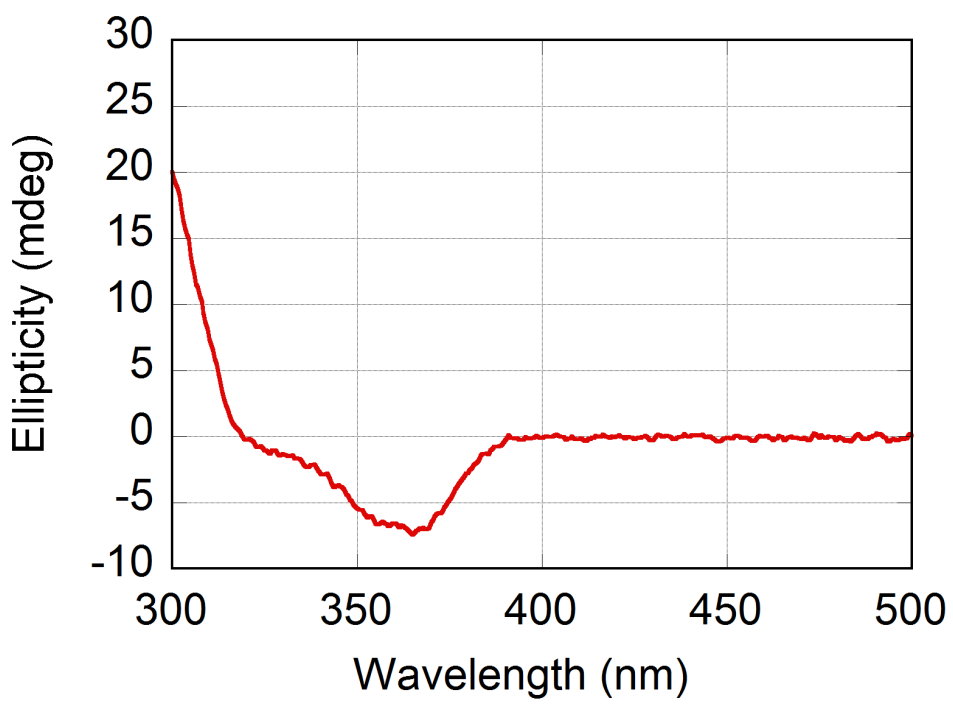

Figure S136. CD spectrum of $\mathbf{3 d}(\mathbf{2 0} / \mathbf{8 0})$ in $\mathrm{PhCH}_{3}\left(1.22 \times 10^{-2} \mathrm{~g} / \mathrm{L}\right.$, light path length $=$ $10 \mathrm{~mm}$ ). 


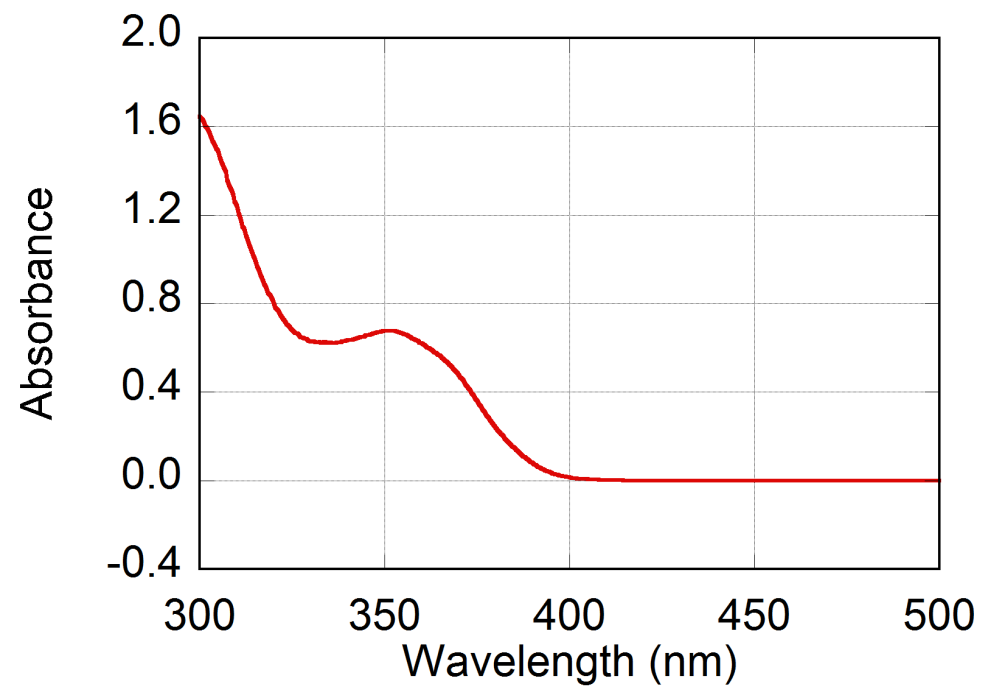

Figure S137. UV-vis absorption spectrum of 3d(30/70) in $\mathrm{PhCH}_{3}\left(2.60 \times 10^{-2} \mathrm{~g} / \mathrm{L}\right.$, light path length $=10 \mathrm{~mm}$ ).

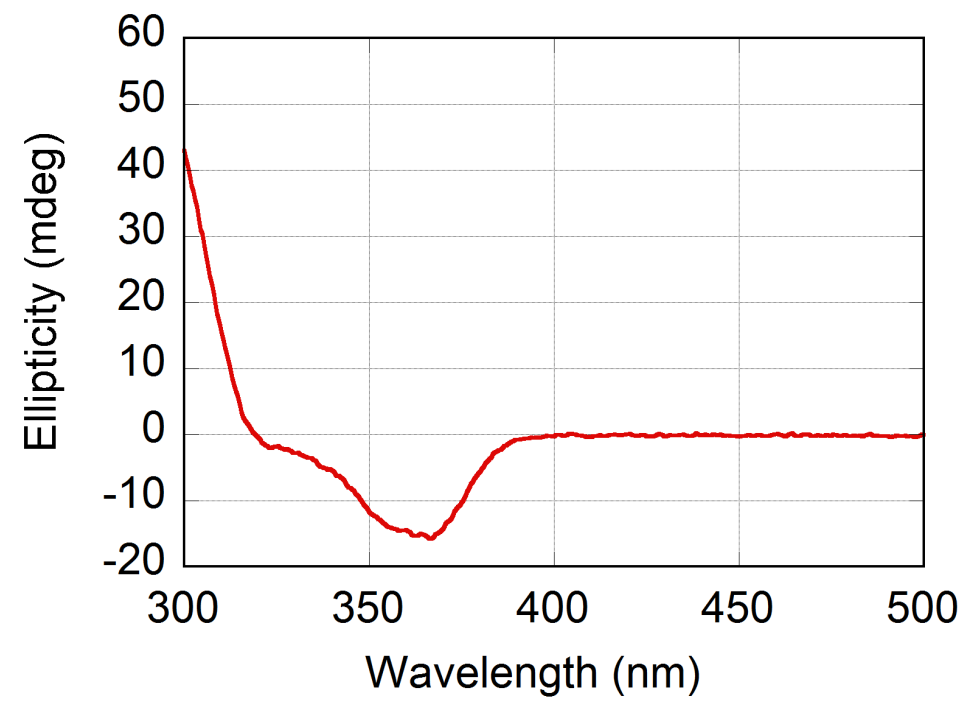

Figure S138. CD spectrum of 3d(30/70) in $\mathrm{PhCH}_{3}\left(2.60 \times 10^{-2} \mathrm{~g} / \mathrm{L}\right.$, light path length $=$ $10 \mathrm{~mm}$ ). 


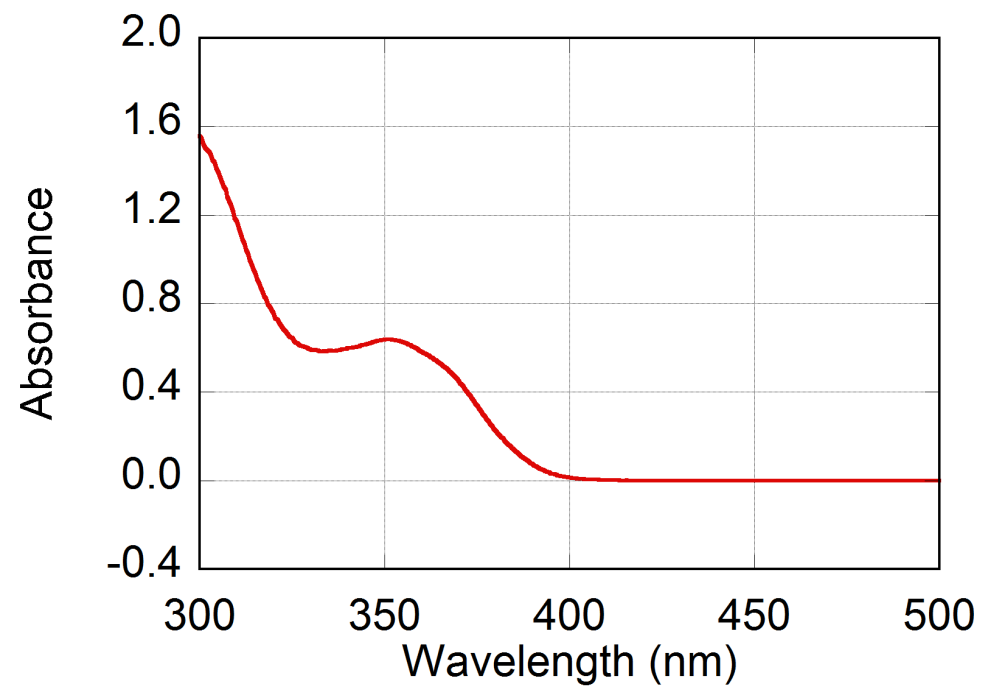

Figure S139. UV-vis absorption spectrum of 3d(40/60) in $\mathrm{PhCH}_{3}\left(2.45 \times 10^{-2} \mathrm{~g} / \mathrm{L}\right.$, light path length $=10 \mathrm{~mm}$ ).

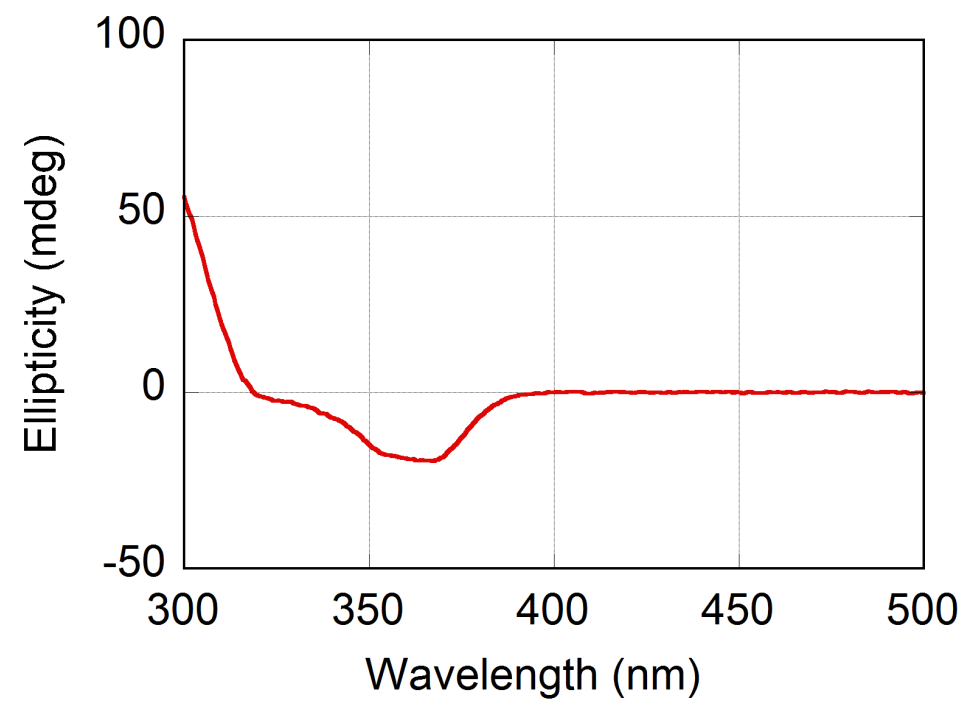

Figure S140. CD spectrum of $\mathbf{3 d}(\mathbf{4 0} / \mathbf{6 0})$ in $\mathrm{PhCH}_{3}\left(2.45 \times 10^{-2} \mathrm{~g} / \mathrm{L}\right.$, light path length $=$ $10 \mathrm{~mm}$ ). 


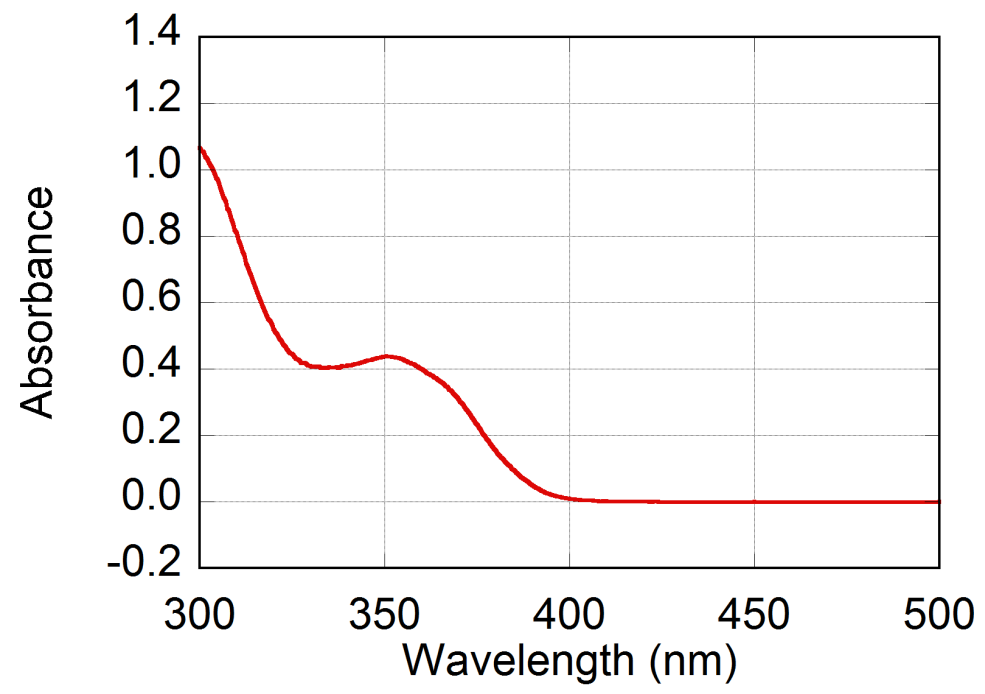

Figure S141. UV-vis absorption spectrum of $\mathbf{3 d}(\mathbf{6 0} / 40)$ in $\mathrm{PhCH}_{3}\left(2.15 \times 10^{-2} \mathrm{~g} / \mathrm{L}\right.$, light path length $=10 \mathrm{~mm}$ ).

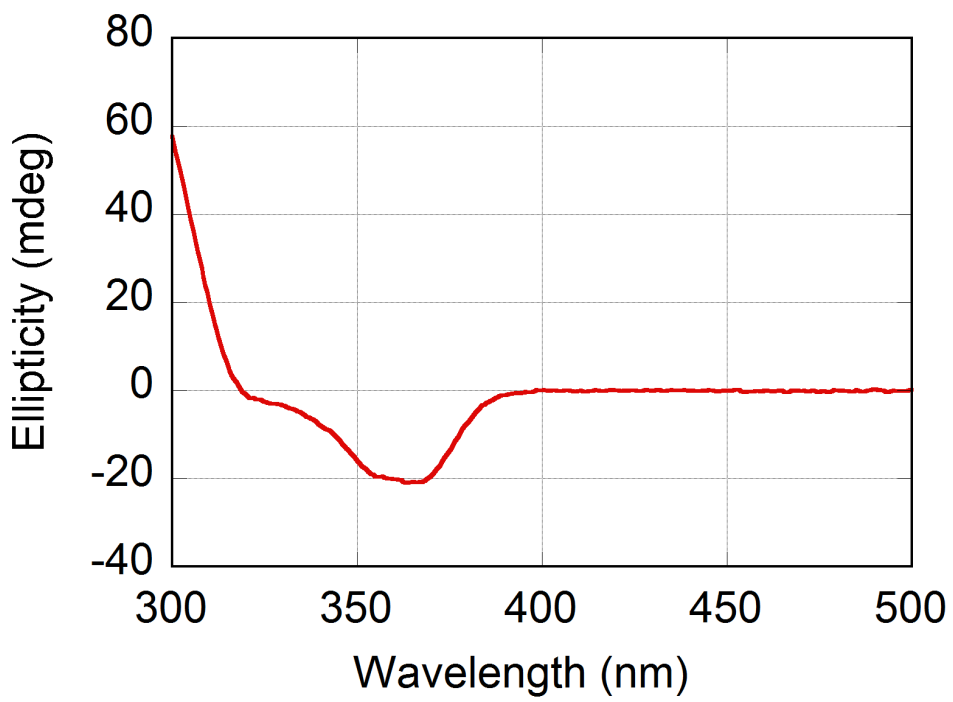

Figure S142. CD spectrum of $\mathbf{3 d}(\mathbf{6 0} / \mathbf{4 0})$ in $\mathrm{PhCH}_{3}\left(2.45 \times 10^{-2} \mathrm{~g} / \mathrm{L}\right.$, light path length $=$ $10 \mathrm{~mm}$ ). 


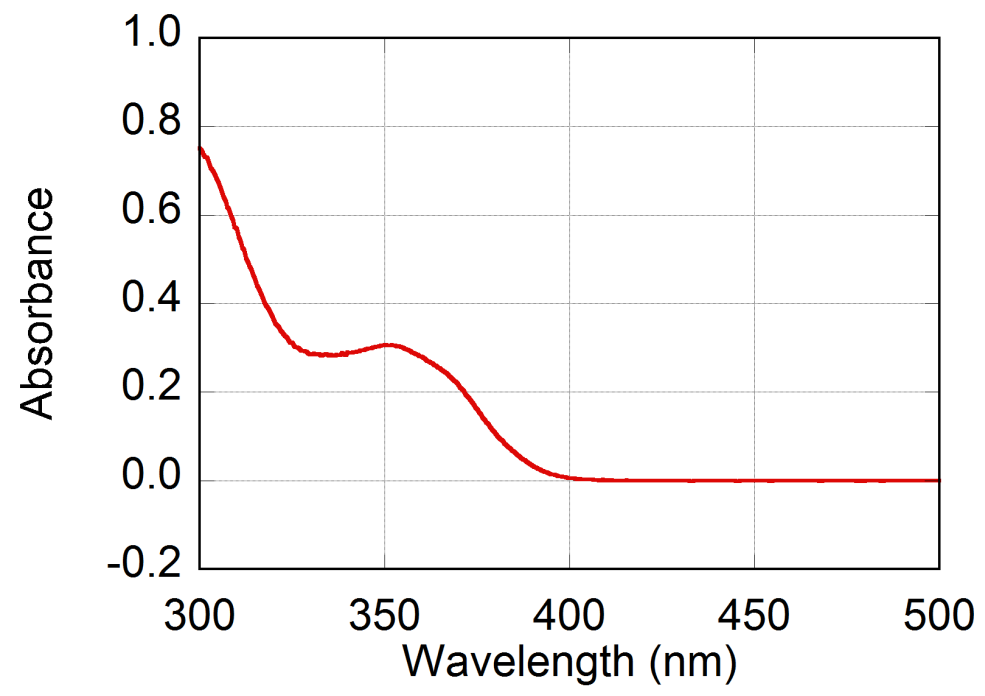

Figure S143. UV-vis absorption spectrum of $\mathbf{3 d}(\mathbf{8 0} / \mathbf{2 0})$ in $\mathrm{PhCH}_{3}\left(1.75 \times 10^{-2} \mathrm{~g} / \mathrm{L}\right.$, light path length $=10 \mathrm{~mm}$ ).

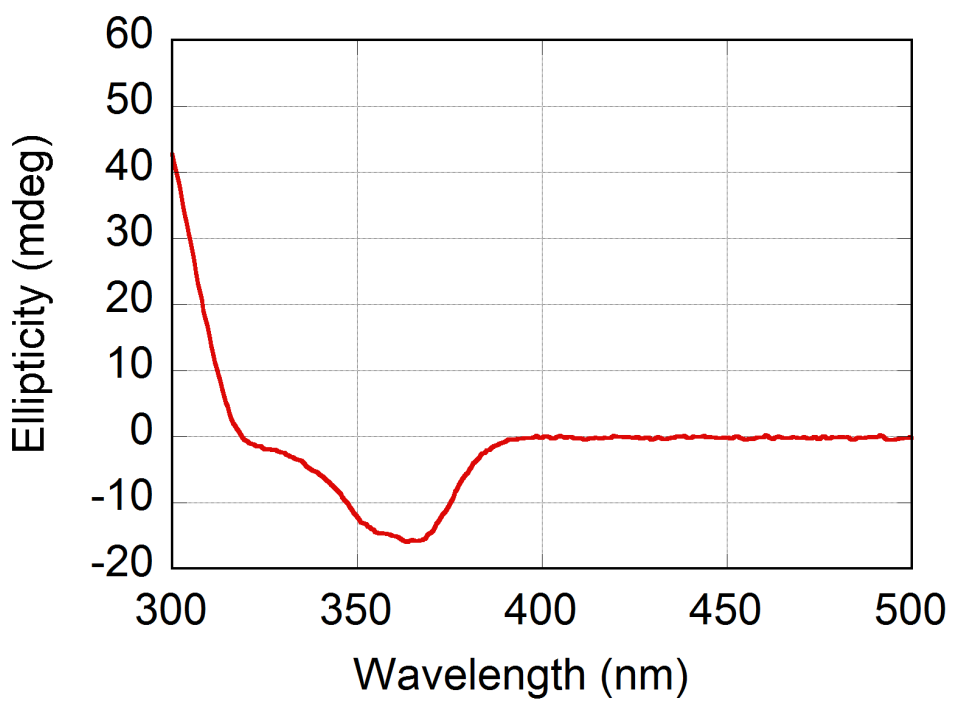

Figure S144. CD spectrum of $\mathbf{3 d}(\mathbf{8 0} / \mathbf{2 0})$ in $\mathrm{PhCH}_{3}\left(1.75 \times 10^{-2} \mathrm{~g} / \mathrm{L}\right.$, light path length $=$ $10 \mathrm{~mm}$ ). 


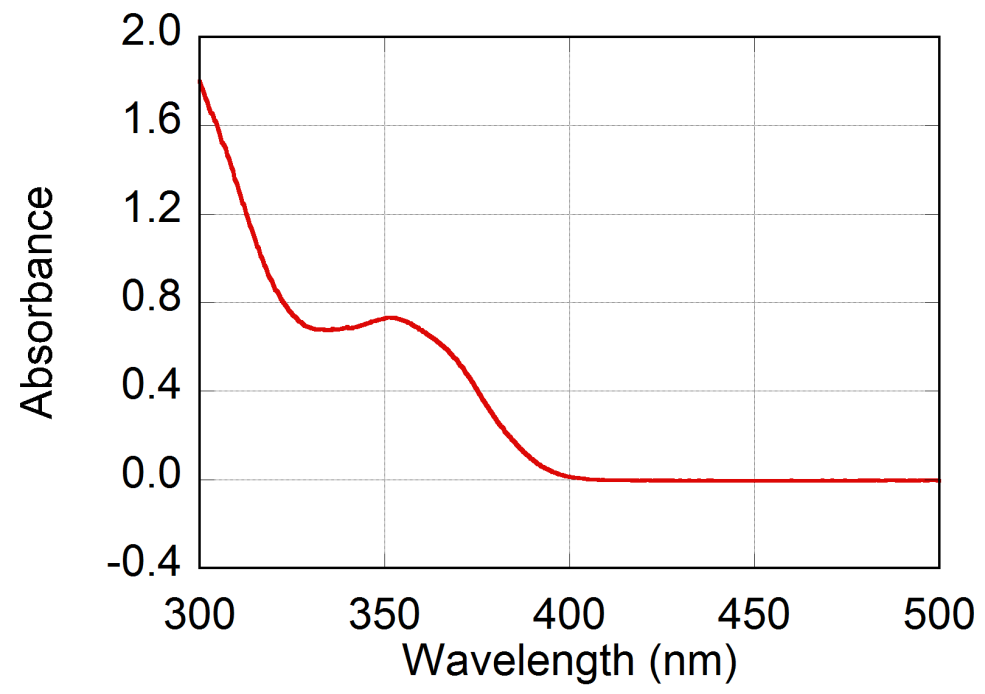

Figure S145. UV-vis absorption spectrum of 3d(2.5/97.5) in $\mathrm{PhOCH}_{3}\left(2.46 \times 10^{-2} \mathrm{~g} / \mathrm{L}\right.$, light path length $=10 \mathrm{~mm}$ ).

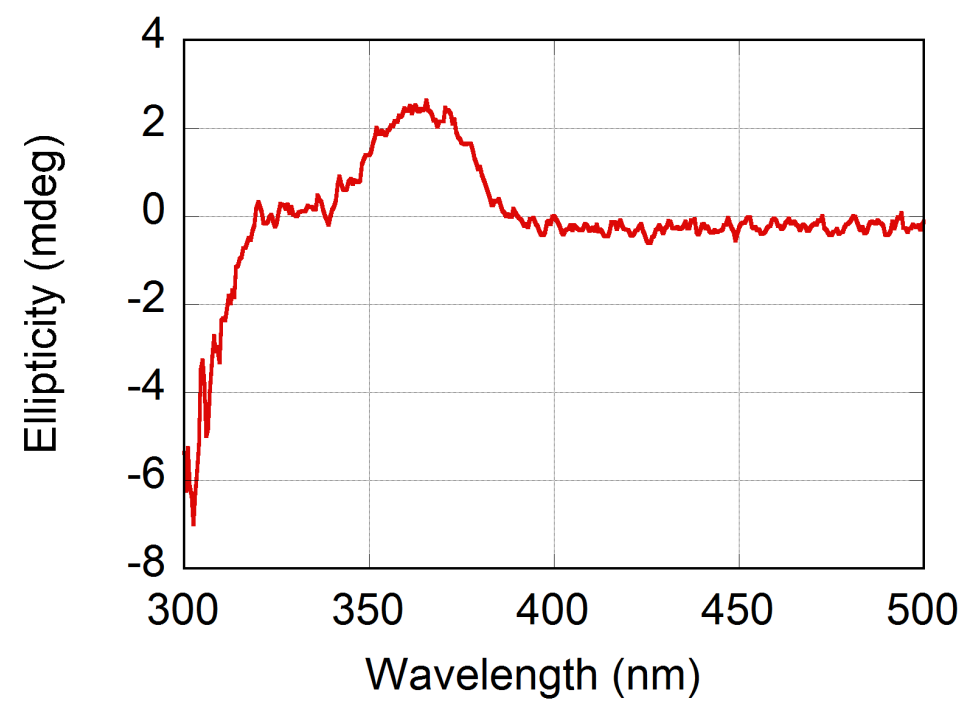

Figure S146. CD spectrum of $\mathbf{3 d}(\mathbf{2 . 5 / 9 7 . 5})$ in $\mathrm{PhOCH}_{3}\left(2.46 \times 10^{-2} \mathrm{~g} / \mathrm{L}\right.$, light path length $=10 \mathrm{~mm}$ ). 


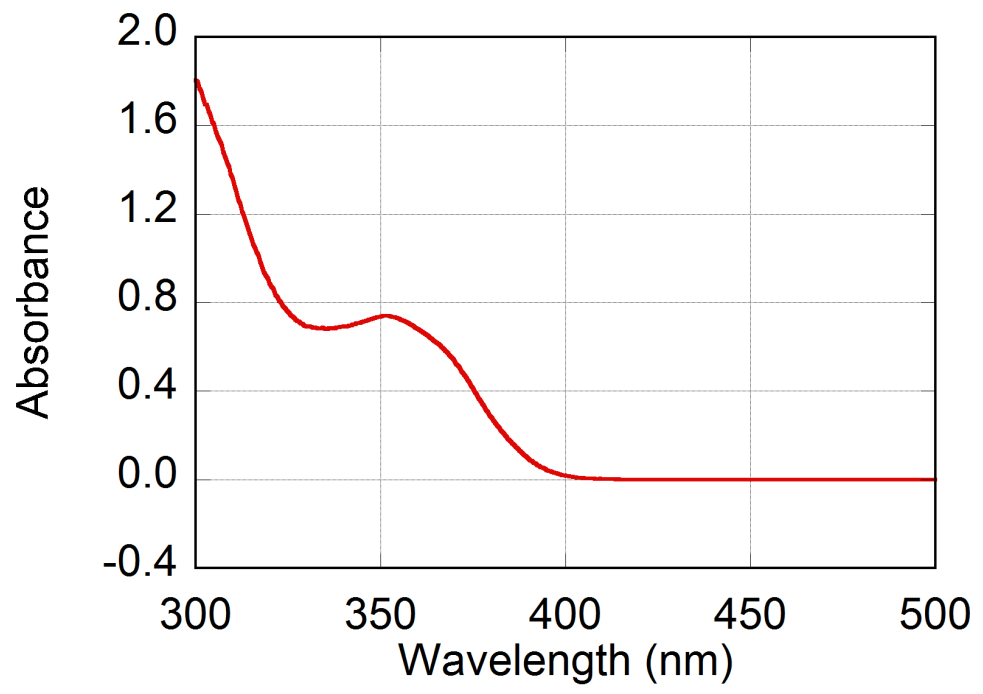

Figure S147. UV-vis absorption spectrum of 3d(5/95) in $\mathrm{PhOCH}_{3}\left(2.55 \times 10^{-2} \mathrm{~g} / \mathrm{L}\right.$, light path length $=10 \mathrm{~mm}$ ).

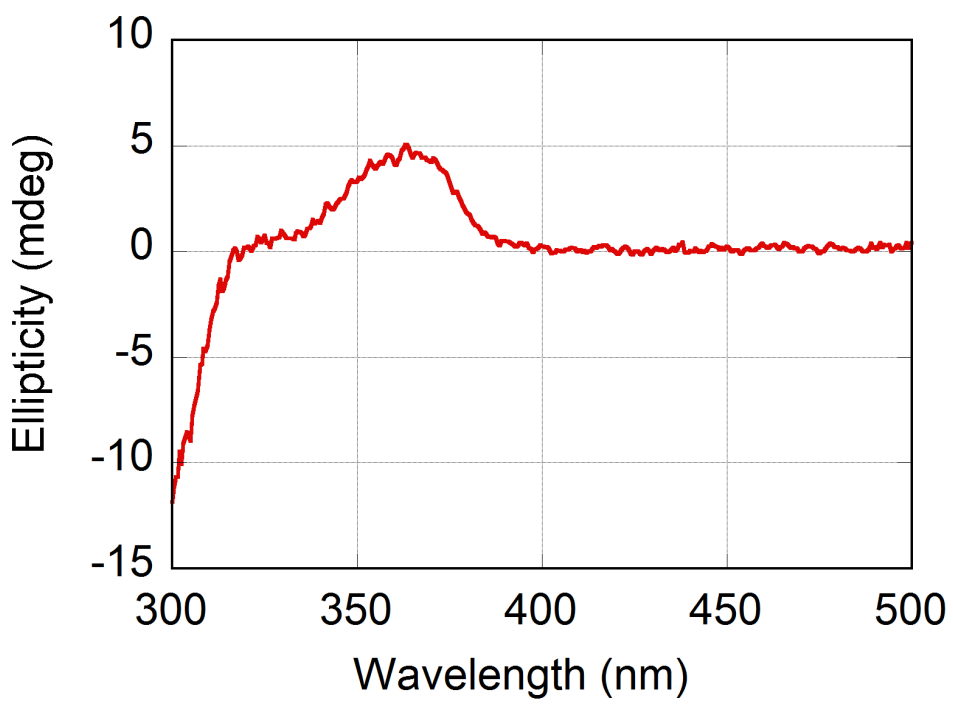

Figure S148. CD spectrum of $\mathbf{3 d}(\mathbf{5} / \mathbf{9 5})$ in $\mathrm{PhOCH}_{3}\left(2.55 \times 10^{-2} \mathrm{~g} / \mathrm{L}\right.$, light path length $=$ $10 \mathrm{~mm}$ ). 


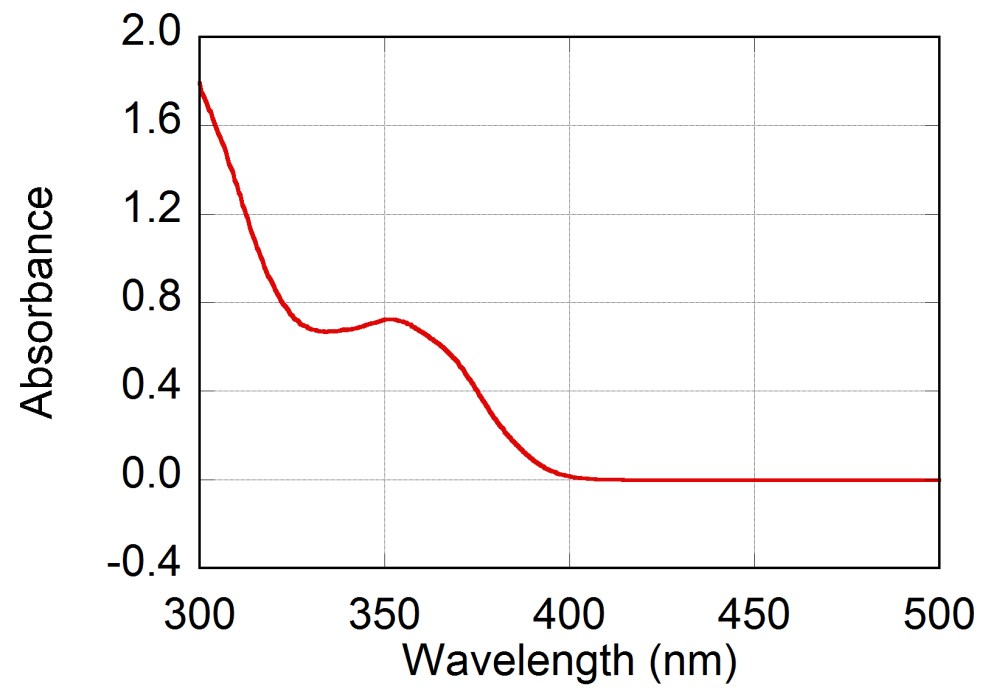

Figure S149. UV-vis absorption spectrum of 3d(7.5/92.5) in $\mathrm{PhOCH}_{3}\left(2.61 \times 10^{-2} \mathrm{~g} / \mathrm{L}\right.$, light path length $=10 \mathrm{~mm}$ ).

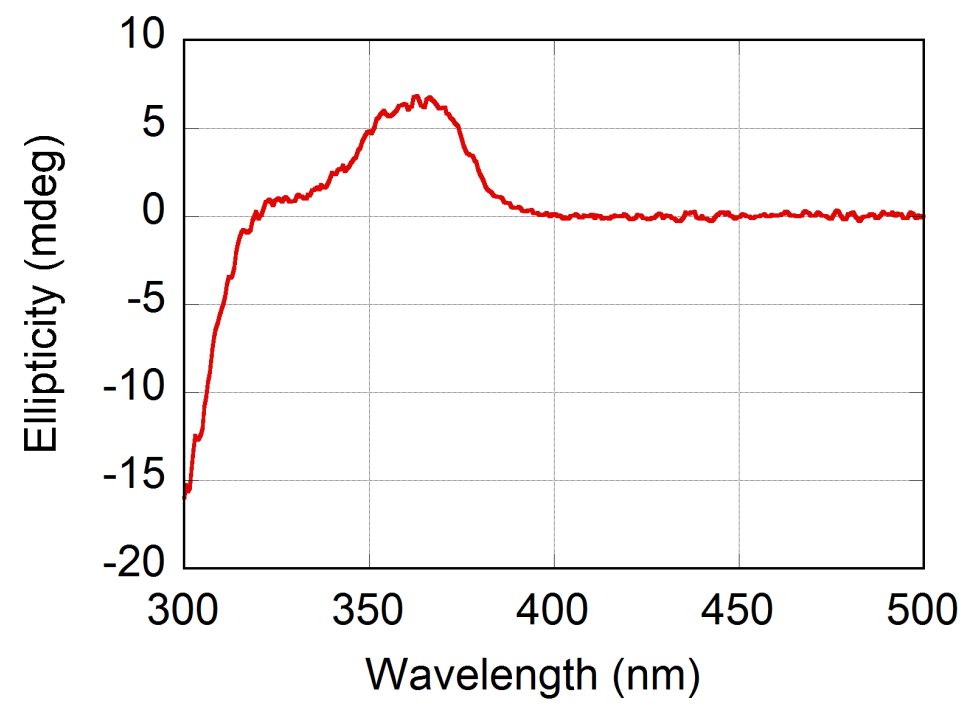

Figure S150. CD spectrum of $\mathbf{3 d}(\mathbf{7 . 5 / 9 2 . 5})$ in $\mathrm{PhOCH}_{3}\left(2.55 \times 10^{-2} \mathrm{~g} / \mathrm{L}\right.$, light path length $=10 \mathrm{~mm}$ ). 


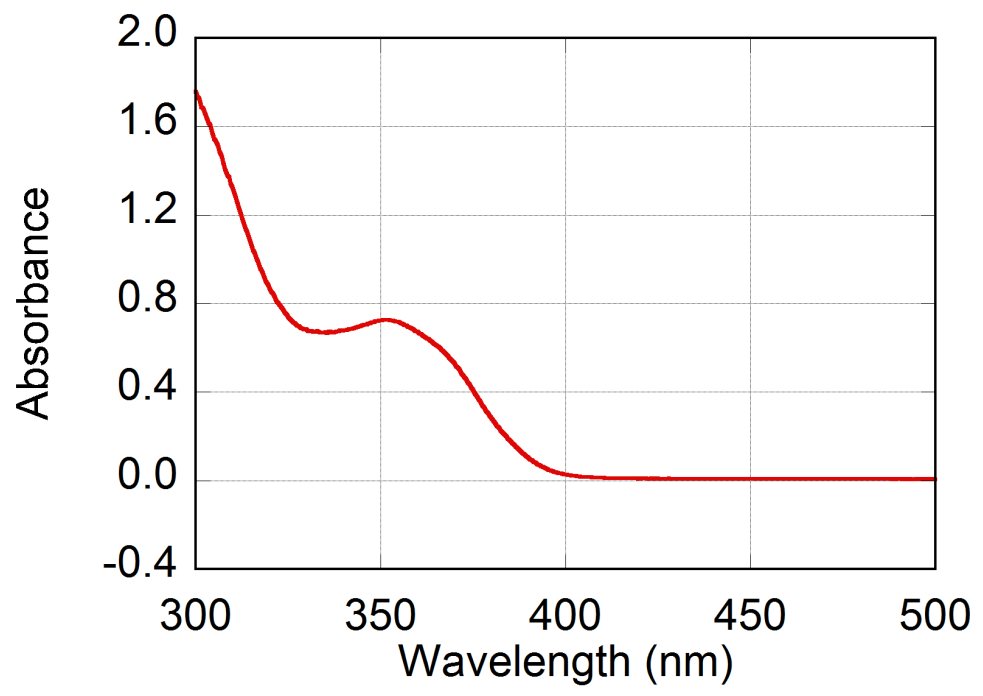

Figure S151. UV-vis absorption spectrum of 3d(10/90) in $\mathrm{PhOCH}_{3}\left(2.55 \times 10^{-2} \mathrm{~g} / \mathrm{L}\right.$, light path length $=10 \mathrm{~mm}$ ).

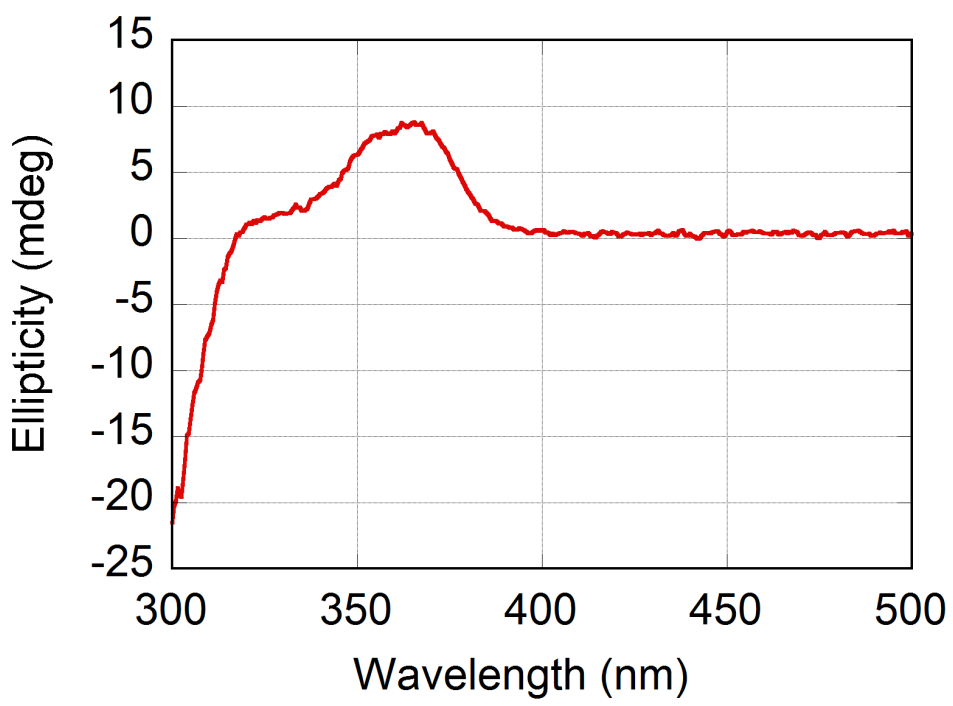

Figure S152. CD spectrum of 3d(10/90) in $\mathrm{PhOCH}_{3}\left(2.55 \times 10^{-2} \mathrm{~g} / \mathrm{L}\right.$, light path length $=10 \mathrm{~mm}$ ). 


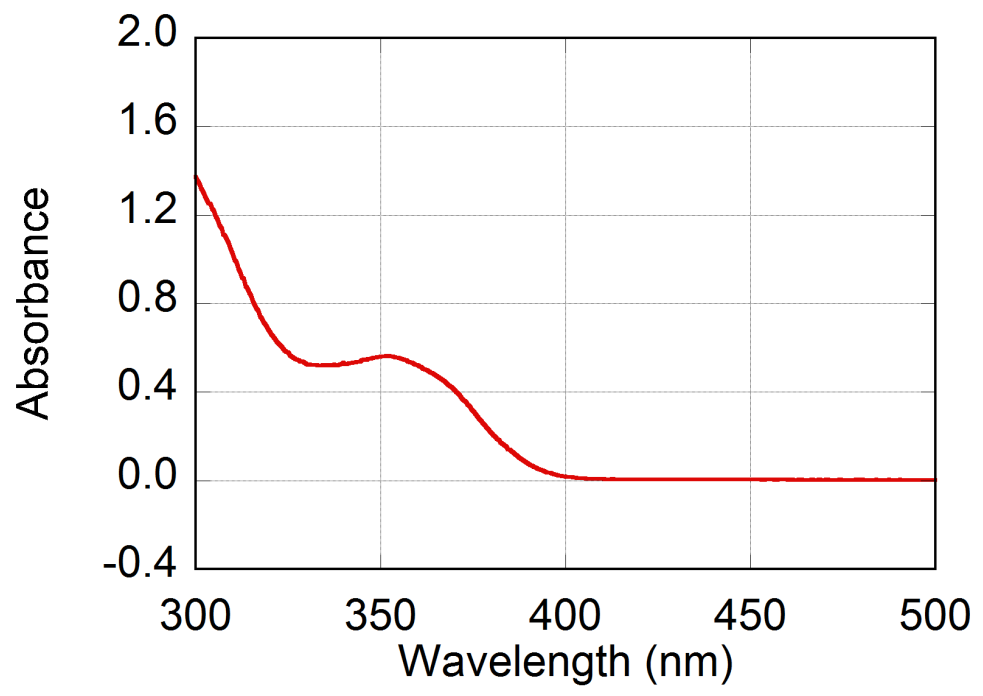

Figure S153. UV-vis absorption spectrum of 3d(15/85) in $\mathrm{PhOCH}_{3}\left(1.88 \times 10^{-2} \mathrm{~g} / \mathrm{L}\right.$, light path length $=10 \mathrm{~mm}$ ).

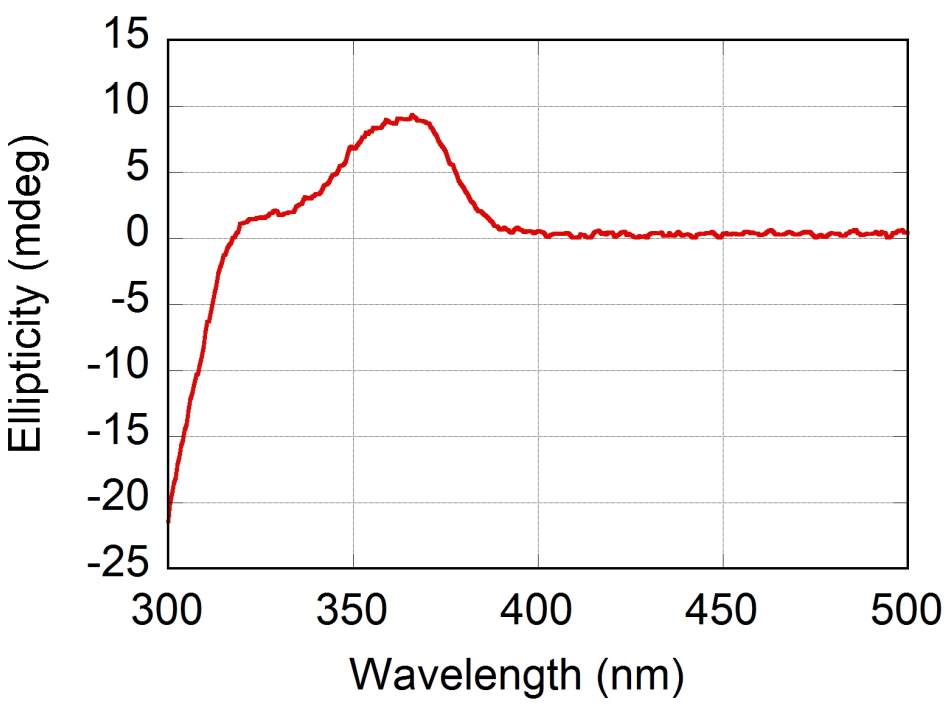

Figure S154. CD spectrum of 3d(15/85) in $\mathrm{PhOCH}_{3}\left(1.88 \times 10^{-2} \mathrm{~g} / \mathrm{L}\right.$, light path length $=10 \mathrm{~mm}$ ). 


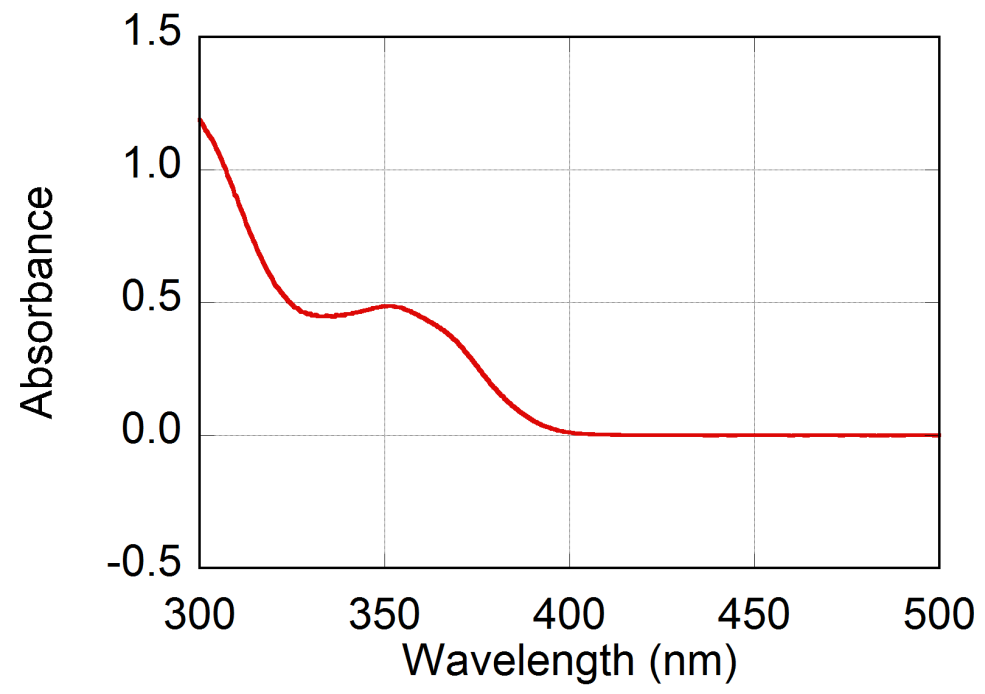

Figure S155. UV-vis absorption spectrum of 3d(20/80) in $\mathrm{PhOCH}_{3}\left(1.22 \times 10^{-2} \mathrm{~g} / \mathrm{L}\right.$, light path length $=10 \mathrm{~mm}$ ).

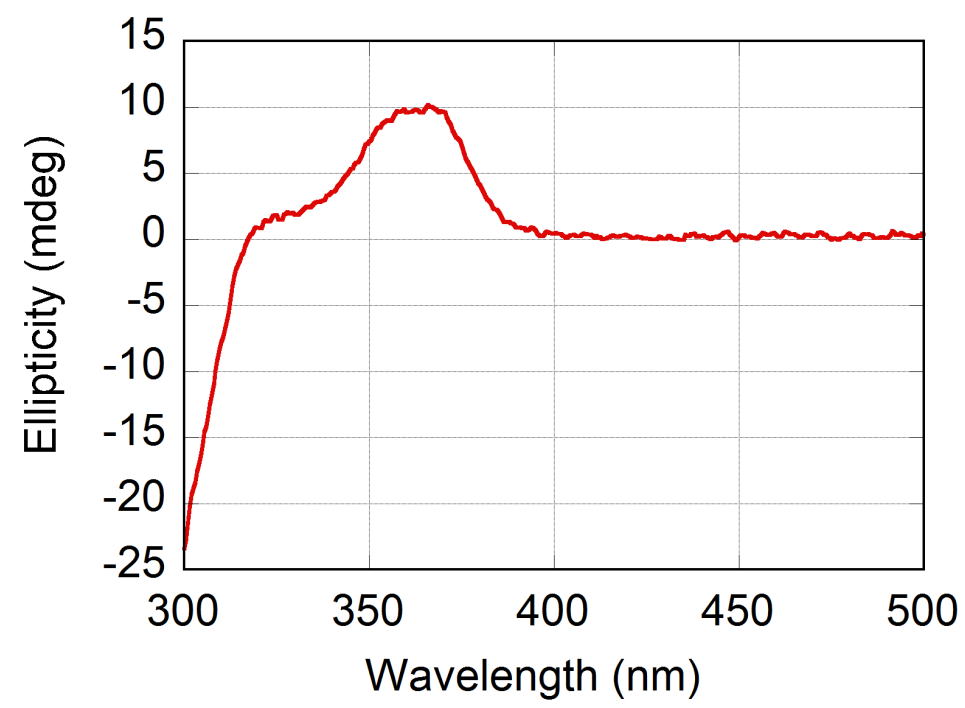

Figure S156. CD spectrum of 3d(20/80) in $\mathrm{PhOCH}_{3}\left(1.22 \times 10^{-2} \mathrm{~g} / \mathrm{L}\right.$, light path length $=10 \mathrm{~mm}$ ). 


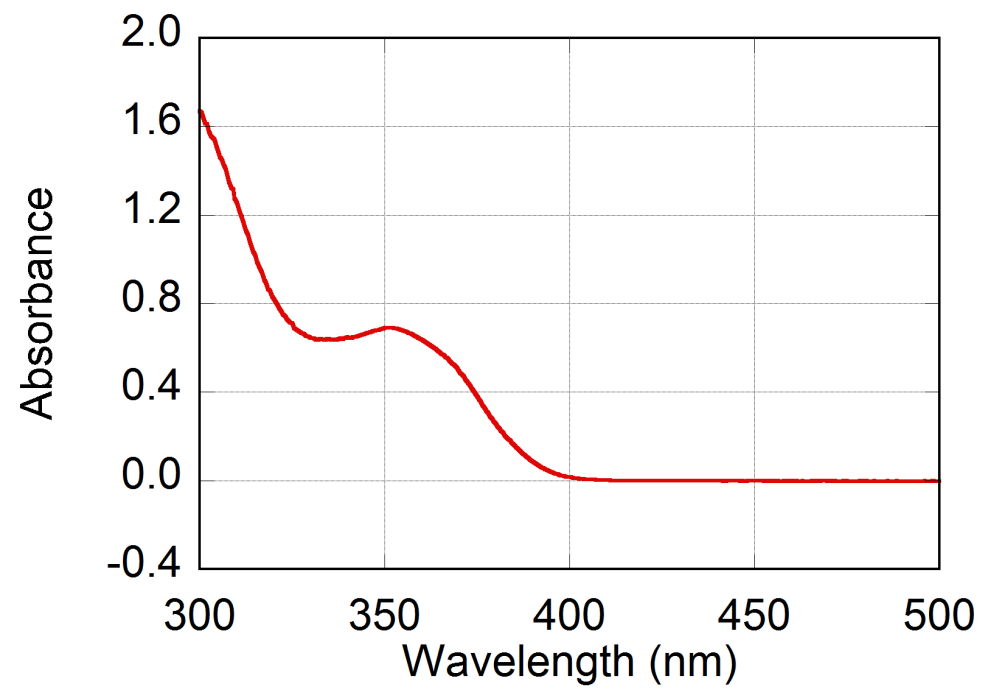

Figure S157. UV-vis absorption spectrum of 3d(30/70) in $\mathrm{PhOCH}_{3}\left(2.60 \times 10^{-2} \mathrm{~g} / \mathrm{L}\right.$, light path length $=10 \mathrm{~mm}$ ).

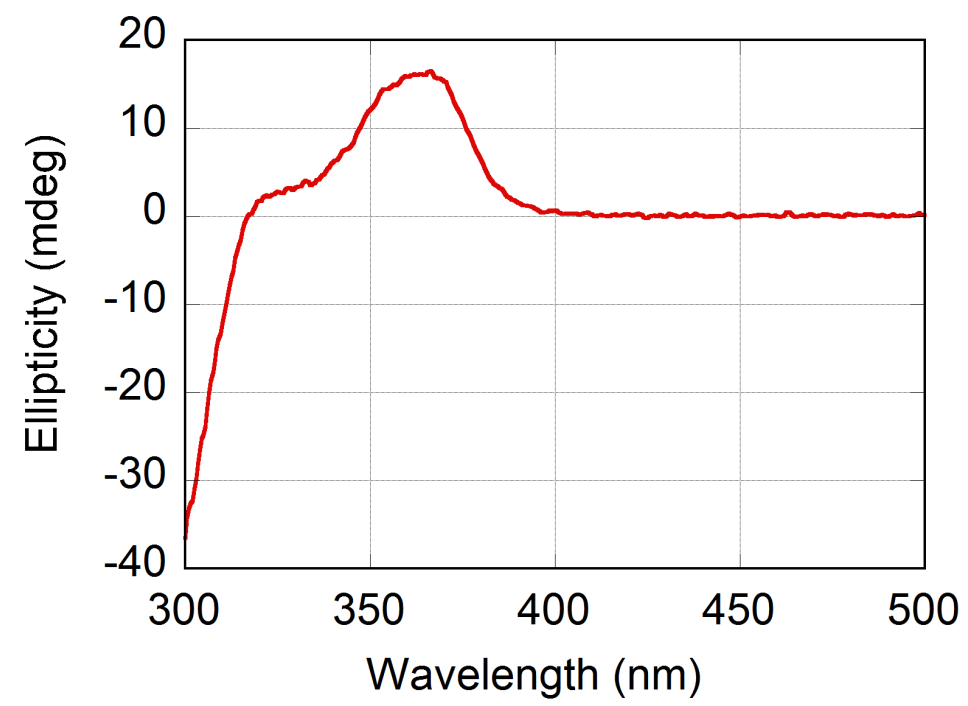

Figure S158. CD spectrum of 3d(30/70) in $\mathrm{PhOCH}_{3}\left(2.60 \times 10^{-2} \mathrm{~g} / \mathrm{L}\right.$, light path length $=10 \mathrm{~mm}$ ). 


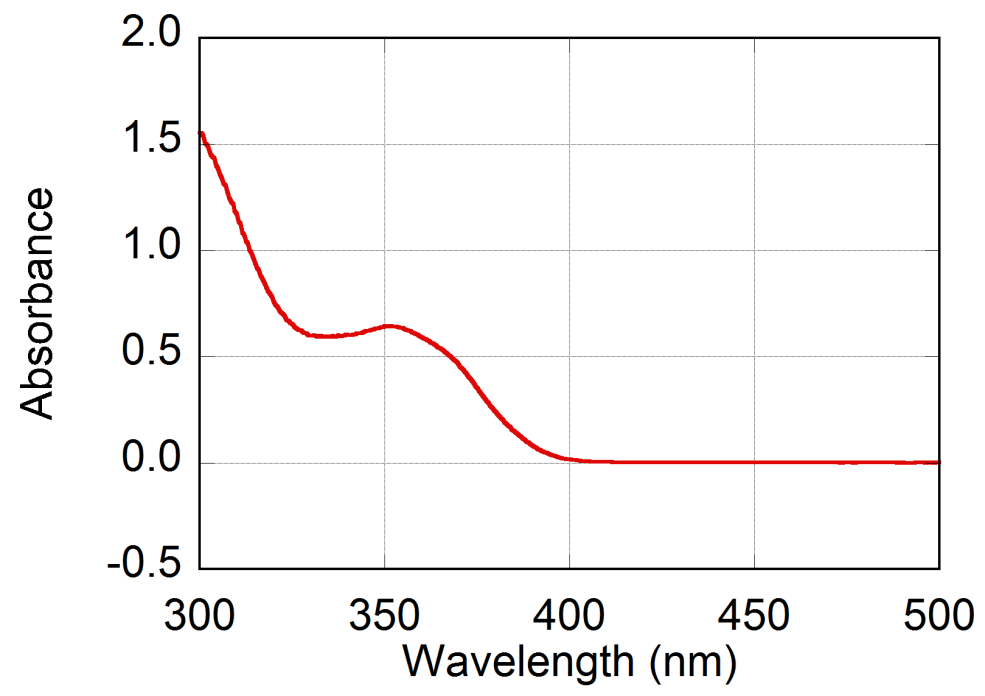

Figure S159. UV-vis absorption spectrum of 3d(40/60) in $\mathrm{PhOCH}_{3}\left(2.45 \times 10^{-2} \mathrm{~g} / \mathrm{L}\right.$, light path length $=10 \mathrm{~mm}$ ).

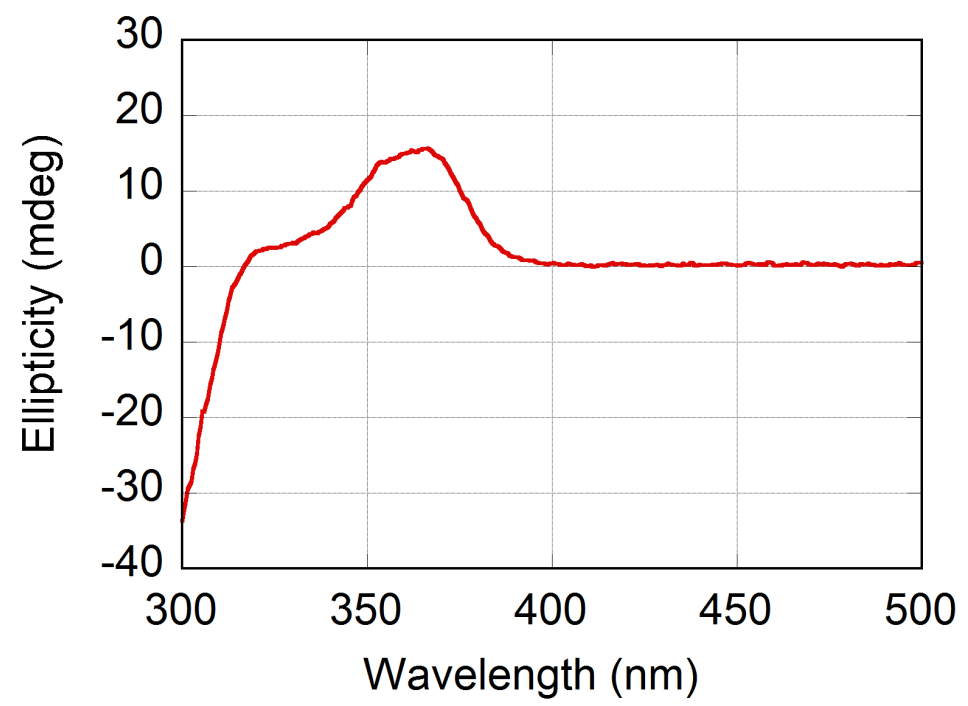

Figure S160. CD spectrum of 3d(40/60) in $\mathrm{PhOCH}_{3}\left(2.45 \times 10^{-2} \mathrm{~g} / \mathrm{L}\right.$, light path length $=10 \mathrm{~mm}$ ). 


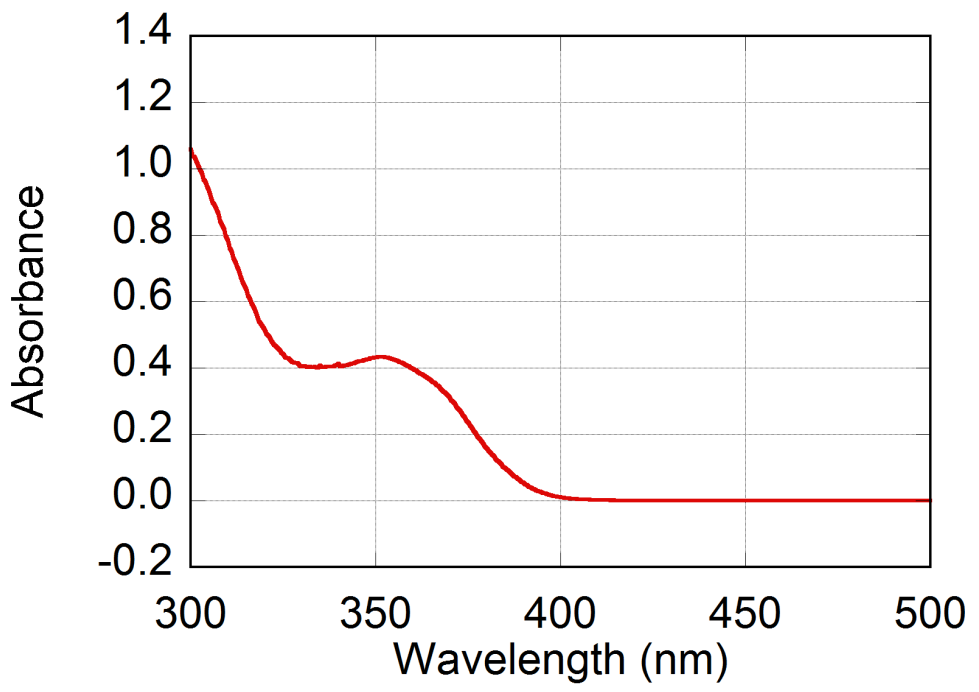

Figure S161. UV-vis absorption spectrum of 3d(60/40) in $\mathrm{PhOCH}_{3}\left(2.15 \times 10^{-2} \mathrm{~g} / \mathrm{L}\right.$, light path length $=10 \mathrm{~mm}$ ).

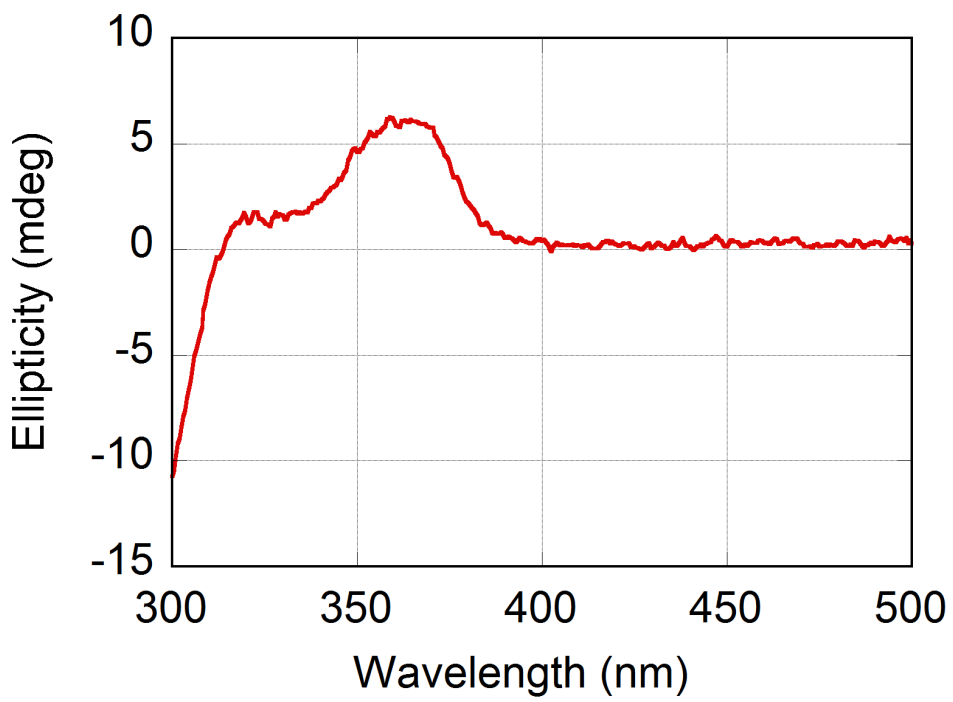

Figure S162. CD spectrum of 3d(60/40) in $\mathrm{PhOCH}_{3}\left(2.15 \times 10^{-2} \mathrm{~g} / \mathrm{L}\right.$, light path length $=10 \mathrm{~mm}$ ). 


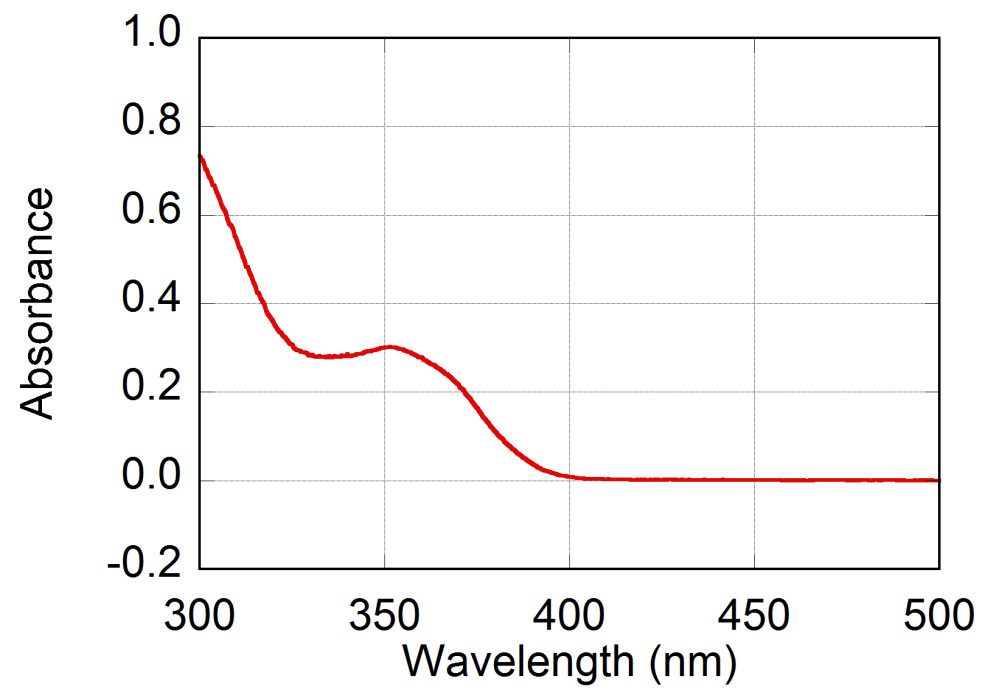

Figure S163. UV-vis absorption spectrum of 3d(80/20) in $\mathrm{PhOCH}_{3}\left(1.75 \times 10^{-2} \mathrm{~g} / \mathrm{L}\right.$, light path length $=10 \mathrm{~mm}$ ).

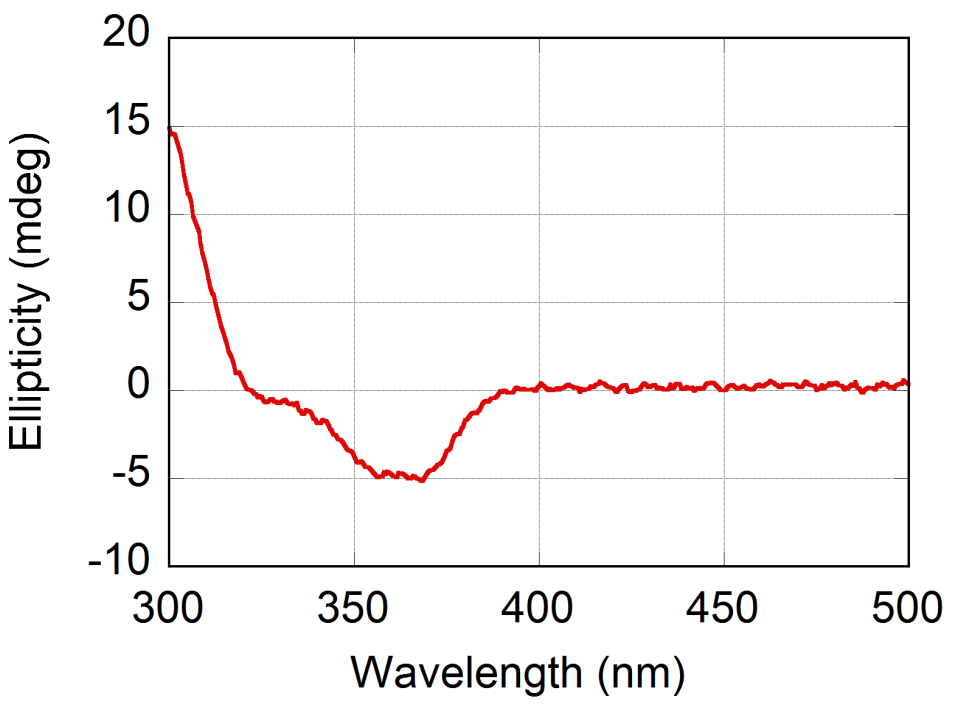

Figure S164. CD spectrum of $\mathbf{3 d}(\mathbf{8 0} / \mathbf{2 0})$ in $\mathrm{PhOCH}_{3}\left(1.75 \times 10^{-2} \mathrm{~g} / \mathrm{L}\right.$, light path length $=10 \mathrm{~mm}$ ). 


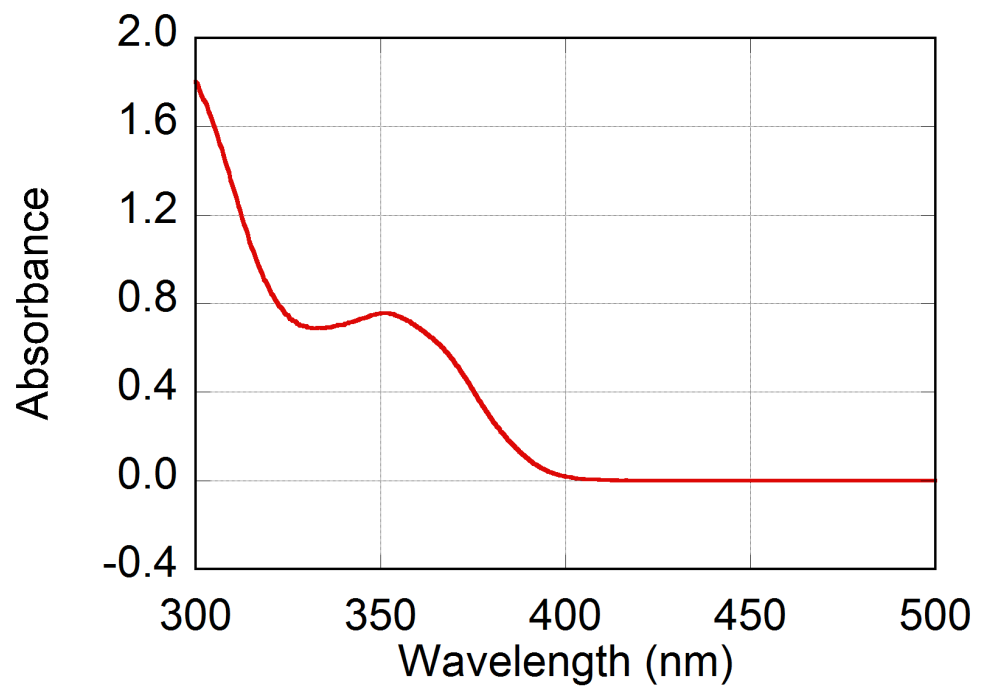

Figure S165. UV-vis absorption spectrum of 3d(2.5/97.5) in $\mathrm{PhCF}_{3}\left(2.46 \times 10^{-2} \mathrm{~g} / \mathrm{L}\right.$, light path length $=10 \mathrm{~mm}$ ).

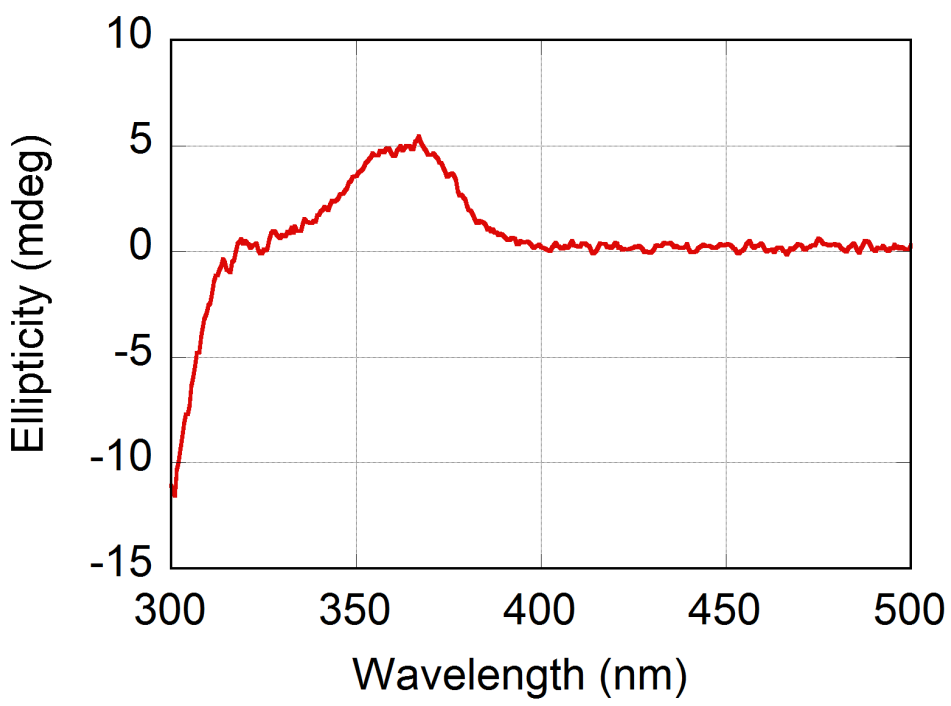

Figure S166. CD spectrum of $\mathbf{3 d}(\mathbf{2 . 5} / \mathbf{9 7 . 5})$ in $\mathrm{PhCF}_{3}\left(2.46 \times 10^{-2} \mathrm{~g} / \mathrm{L}\right.$, light path length $=10 \mathrm{~mm}$ ). 


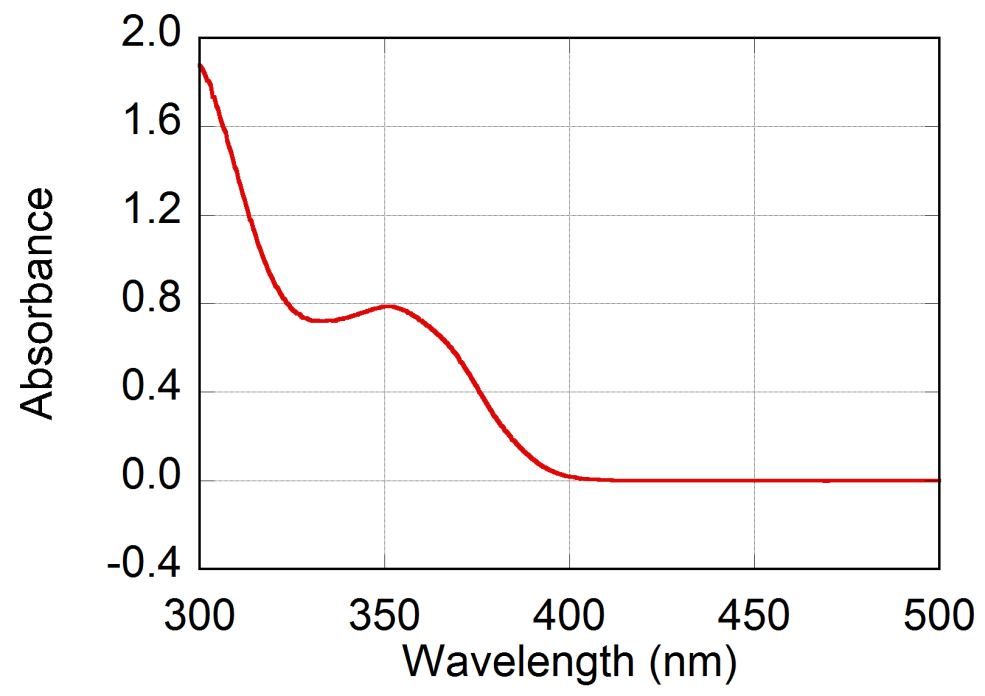

Figure S167. UV-vis absorption spectrum of 3d(5/95) in $\mathrm{PhCF}_{3}\left(2.55 \times 10^{-2} \mathrm{~g} / \mathrm{L}\right.$, light path length $=10 \mathrm{~mm}$ ).

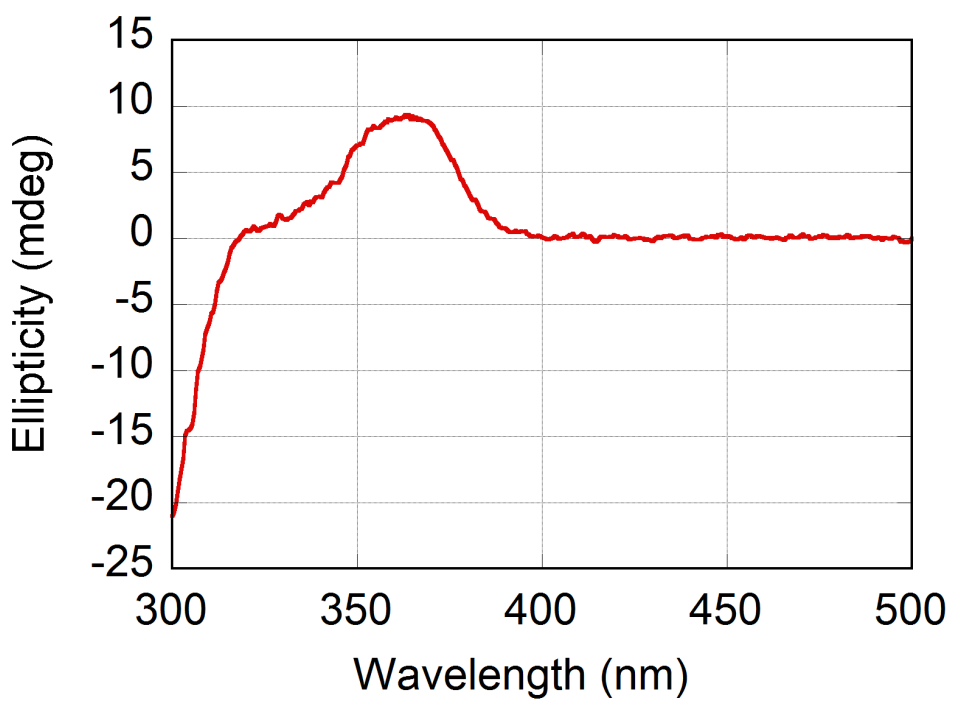

Figure S168. CD spectrum of $\mathbf{3 d}(\mathbf{5} / \mathbf{9 5})$ in $\mathrm{PhCF}_{3}\left(2.55 \times 10^{-2} \mathrm{~g} / \mathrm{L}\right.$, light path length $=10$ $\mathrm{mm})$. 


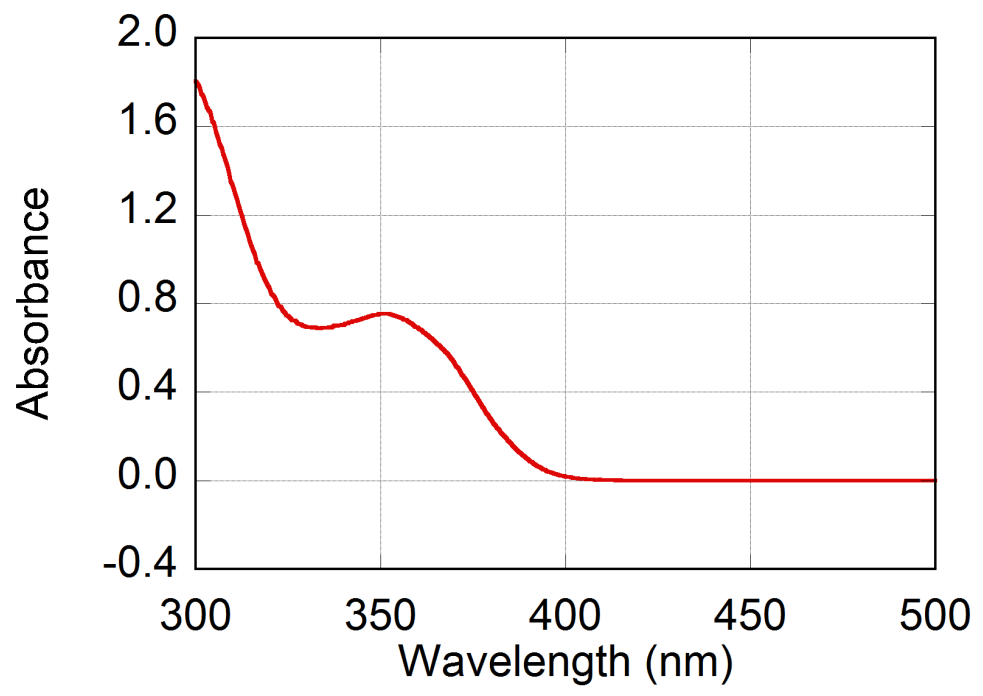

Figure S169. UV-vis absorption spectrum of 3d(7.5/92.5) in $\mathrm{PhCF}_{3}\left(2.61 \times 10^{-2} \mathrm{~g} / \mathrm{L}\right.$, light path length $=10 \mathrm{~mm}$ ).

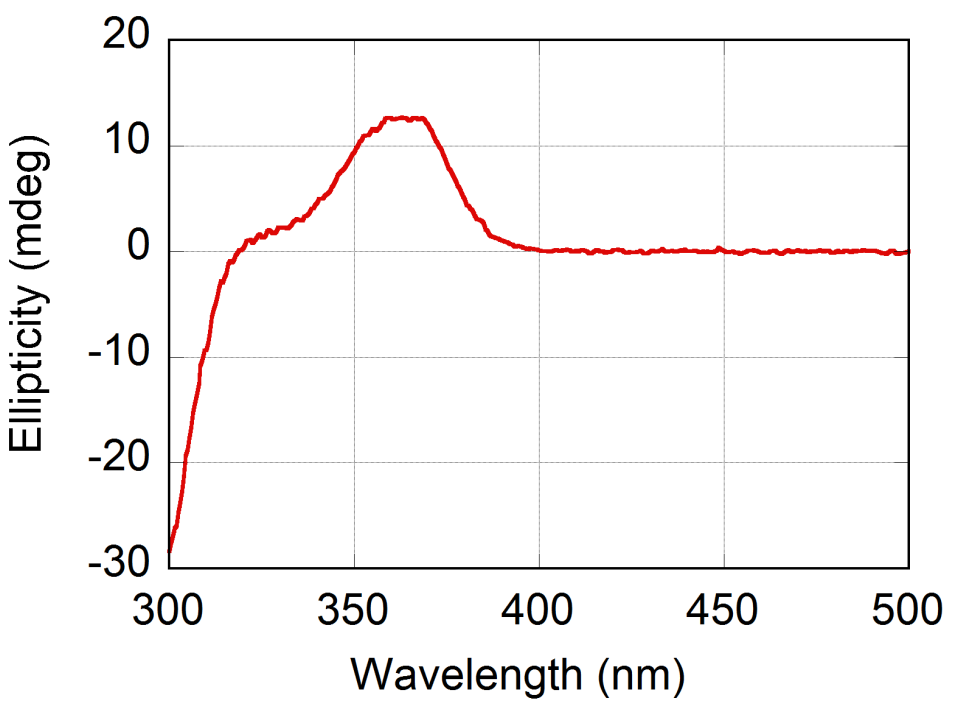

Figure S170. CD spectrum of 3d(7.5/92.5) in $\mathrm{PhCF}_{3}\left(2.61 \times 10^{-2} \mathrm{~g} / \mathrm{L}\right.$, light path length $=10 \mathrm{~mm}$ ). 


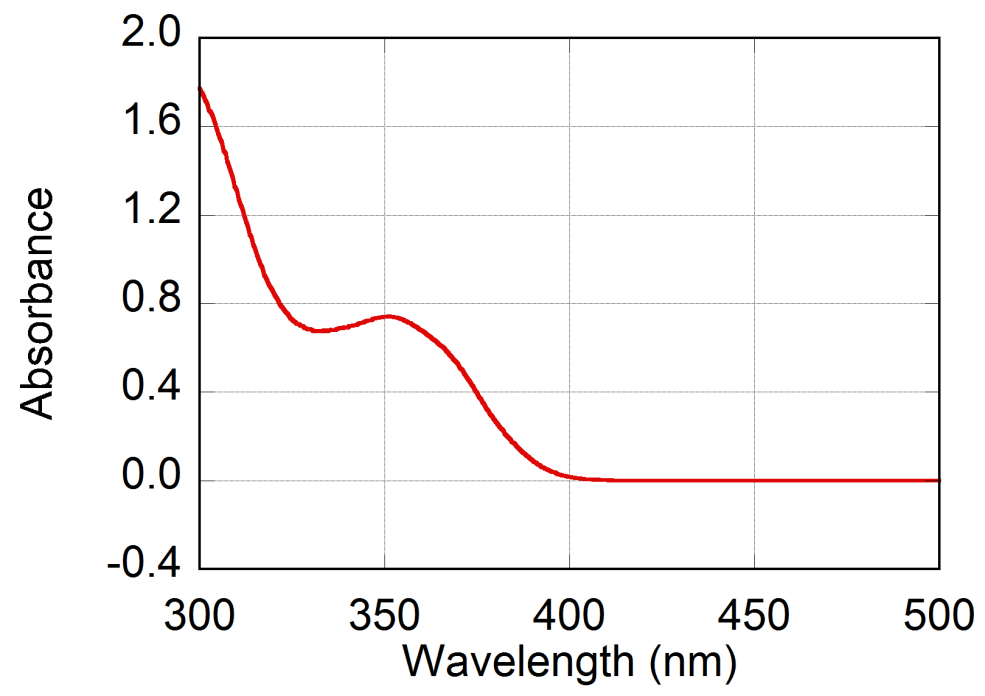

Figure S171. UV-vis absorption spectrum of 3d(10/90) in $\mathrm{PhCF}_{3}\left(2.55 \times 10^{-2} \mathrm{~g} / \mathrm{L}\right.$, light path length $=10 \mathrm{~mm}$ ).

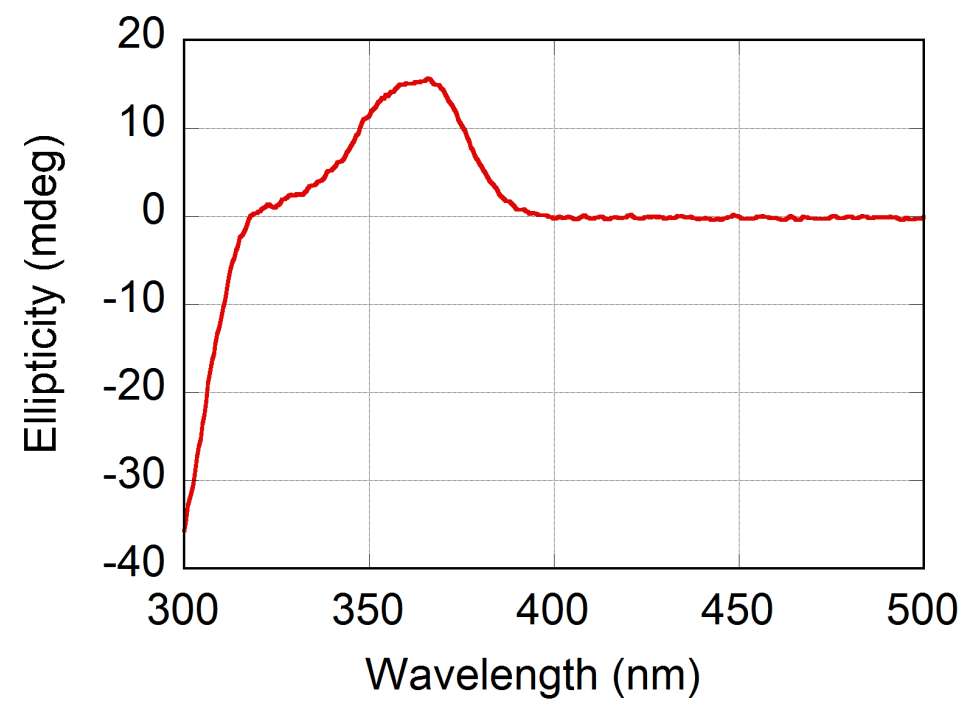

Figure S172. CD spectrum of 3d(10/90) in $\mathrm{PhCF}_{3}\left(2.55 \times 10^{-2} \mathrm{~g} / \mathrm{L}\right.$, light path length $=$ $10 \mathrm{~mm}$ ). 


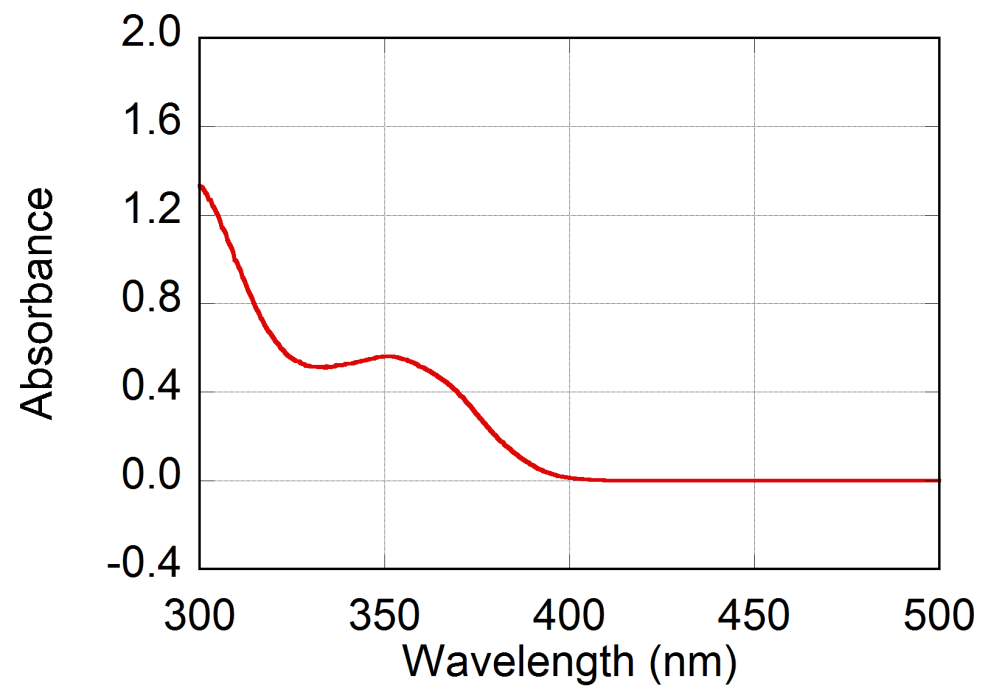

Figure S173. UV-vis absorption spectrum of $\mathbf{3 d}(\mathbf{1 5} / \mathbf{8 5})$ in $\mathrm{PhCF}_{3}\left(1.88 \times 10^{-2} \mathrm{~g} / \mathrm{L}\right.$, light path length $=10 \mathrm{~mm}$ ).

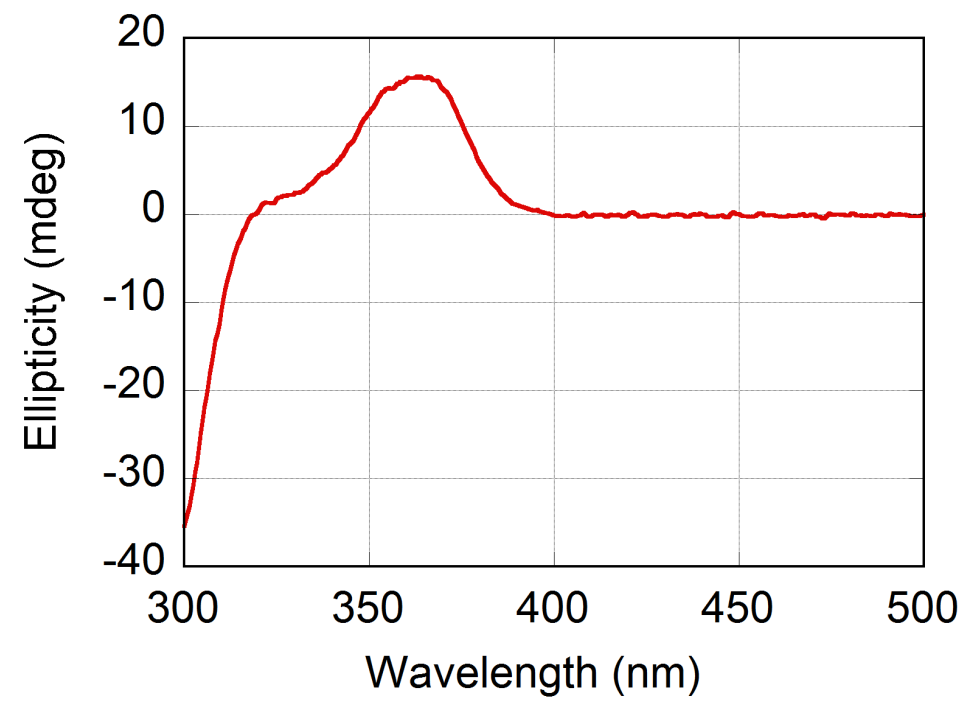

Figure S174. CD spectrum of 3d(15/85) in $\mathrm{PhCF}_{3}\left(1.88 \times 10^{-2} \mathrm{~g} / \mathrm{L}\right.$, light path length $=$ $10 \mathrm{~mm}$ ). 


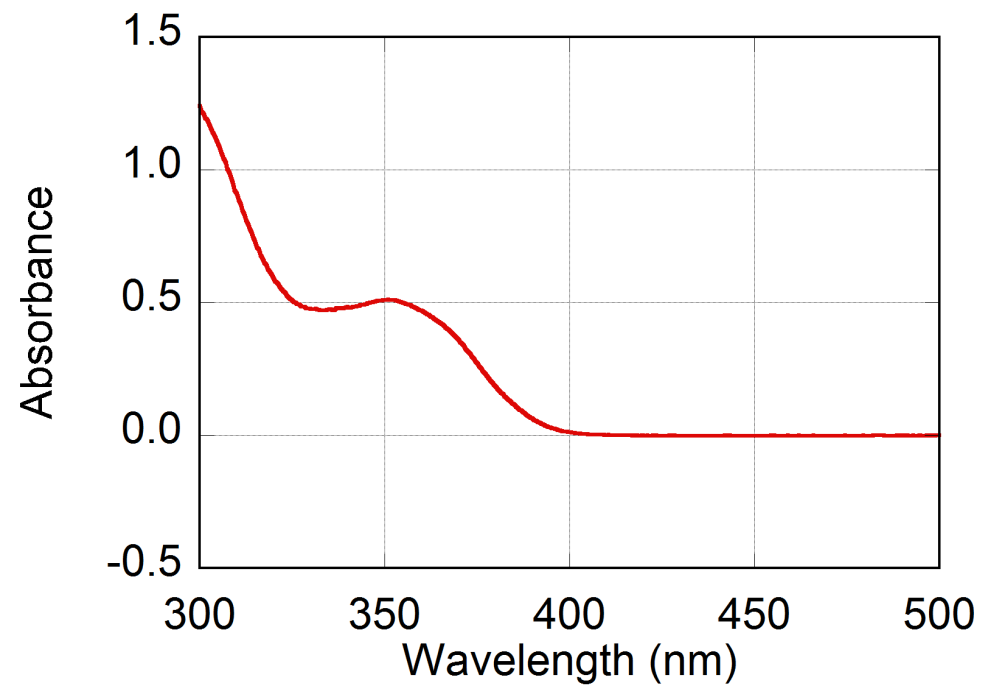

Figure S175. UV-vis absorption spectrum of 3d(20/80) in $\mathrm{PhCF}_{3}\left(1.22 \times 10^{-2} \mathrm{~g} / \mathrm{L}\right.$, light path length $=10 \mathrm{~mm}$ ).

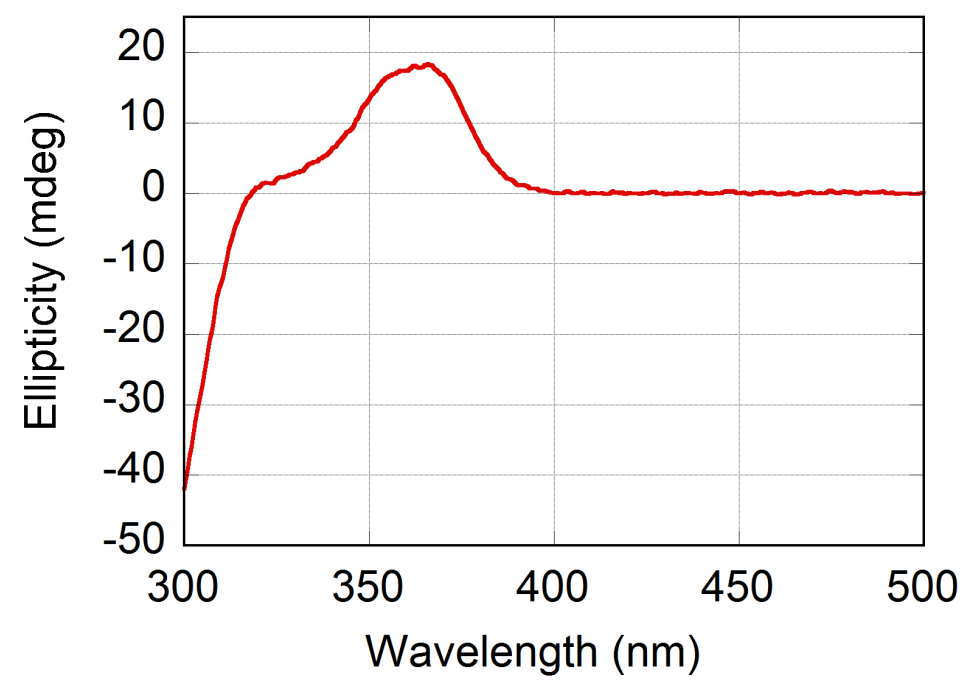

Figure S176. CD spectrum of 3d(20/80) in $\mathrm{PhCF}_{3}\left(1.88 \times 10^{-2} \mathrm{~g} / \mathrm{L}\right.$, light path length $=$ $10 \mathrm{~mm}$ ). 


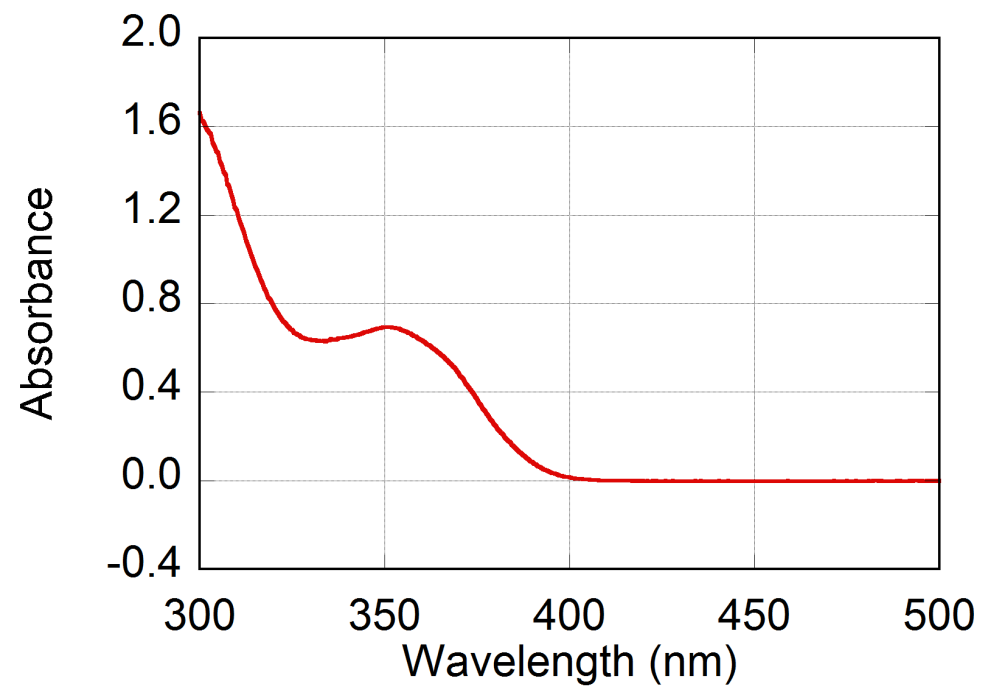

Figure S177. UV-vis absorption spectrum of 3d(30/70) in $\mathrm{PhCF}_{3}\left(2.60 \times 10^{-2} \mathrm{~g} / \mathrm{L}\right.$, light path length $=10 \mathrm{~mm}$ ).

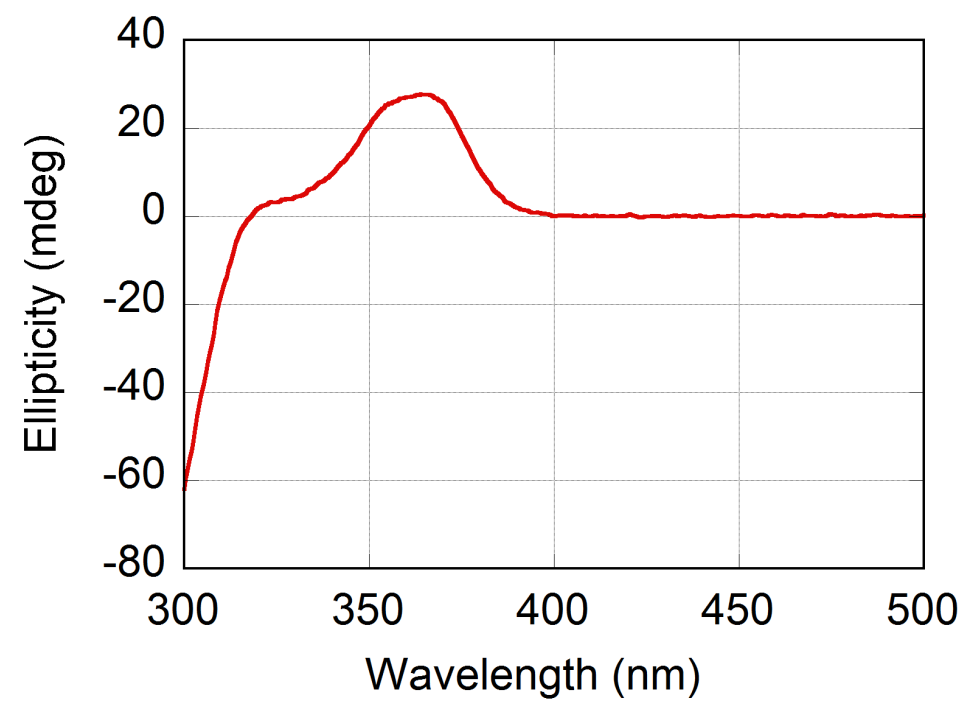

Figure S178. CD spectrum of 3d(30/70) in $\mathrm{PhCF}_{3}\left(2.60 \times 10^{-2} \mathrm{~g} / \mathrm{L}\right.$, light path length $=$ $10 \mathrm{~mm}$ ). 


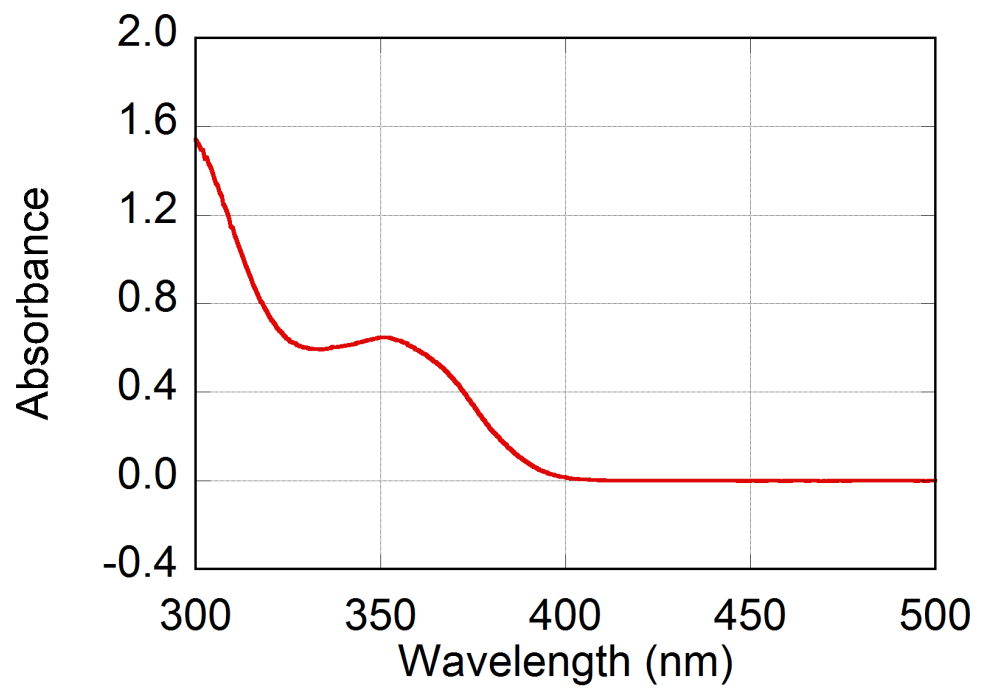

Figure S179. UV-vis absorption spectrum of 3d(40/60) in $\mathrm{PhCF}_{3}\left(2.45 \times 10^{-2} \mathrm{~g} / \mathrm{L}\right.$, light path length $=10 \mathrm{~mm}$ ).

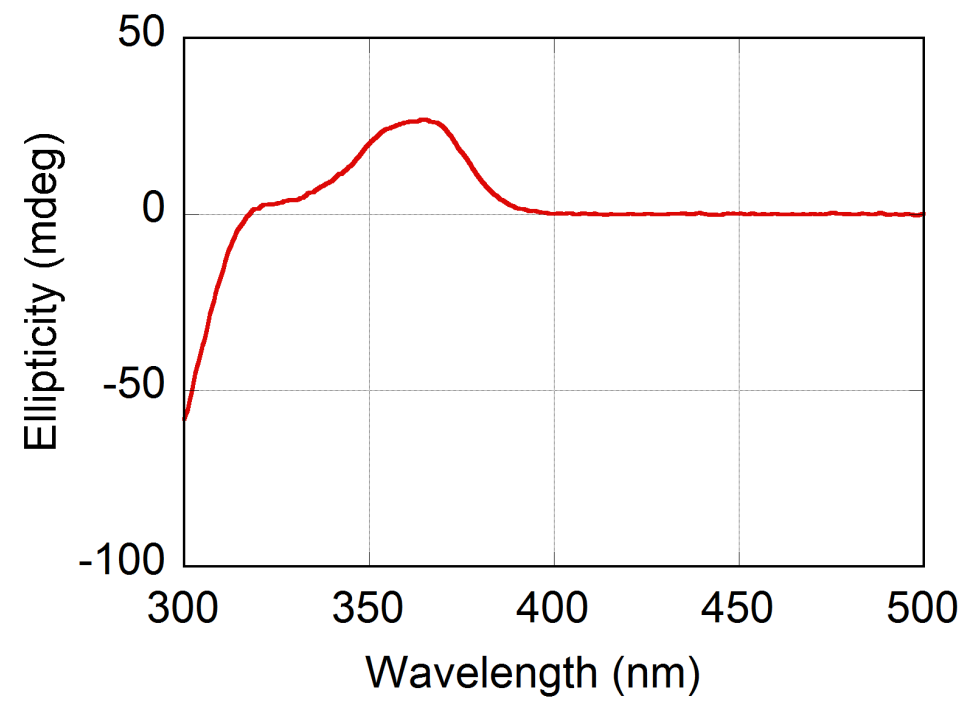

Figure S180. CD spectrum of 3d(40/60) in $\mathrm{PhCF}_{3}\left(2.45 \times 10^{-2} \mathrm{~g} / \mathrm{L}\right.$, light path length $=$ $10 \mathrm{~mm}$ ). 


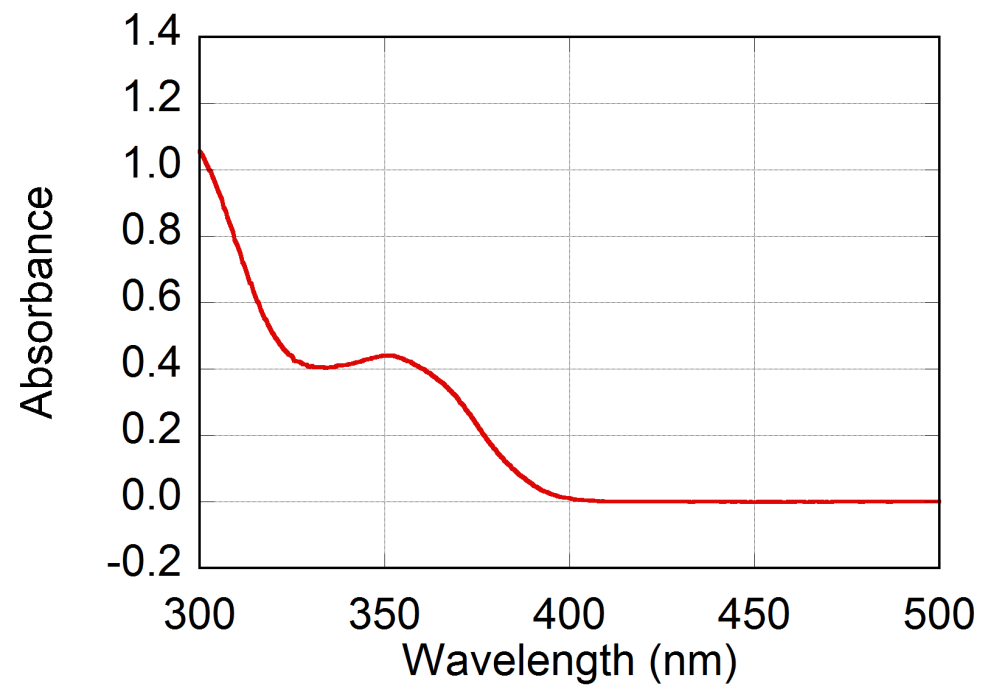

Figure S181. UV-vis absorption spectrum of 3d(60/40) in $\mathrm{PhCF}_{3}\left(2.15 \times 10^{-2} \mathrm{~g} / \mathrm{L}\right.$, light path length $=10 \mathrm{~mm}$ ).

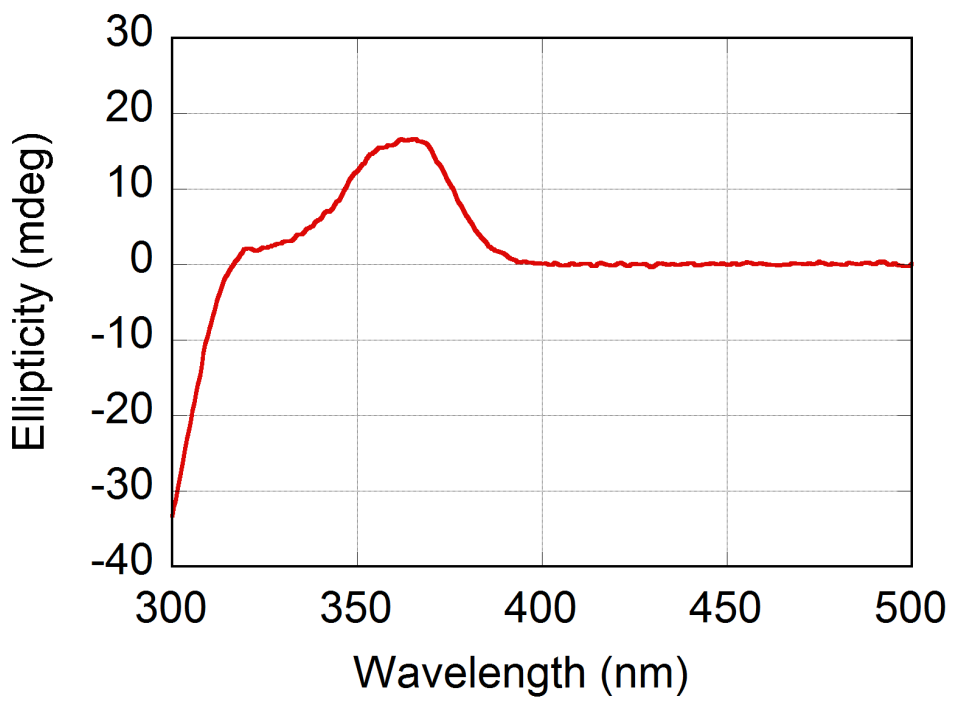

Figure S182. CD spectrum of 3d(60/40) in $\mathrm{PhCF}_{3}\left(2.15 \times 10^{-2} \mathrm{~g} / \mathrm{L}\right.$, light path length $=$ $10 \mathrm{~mm}$ ). 


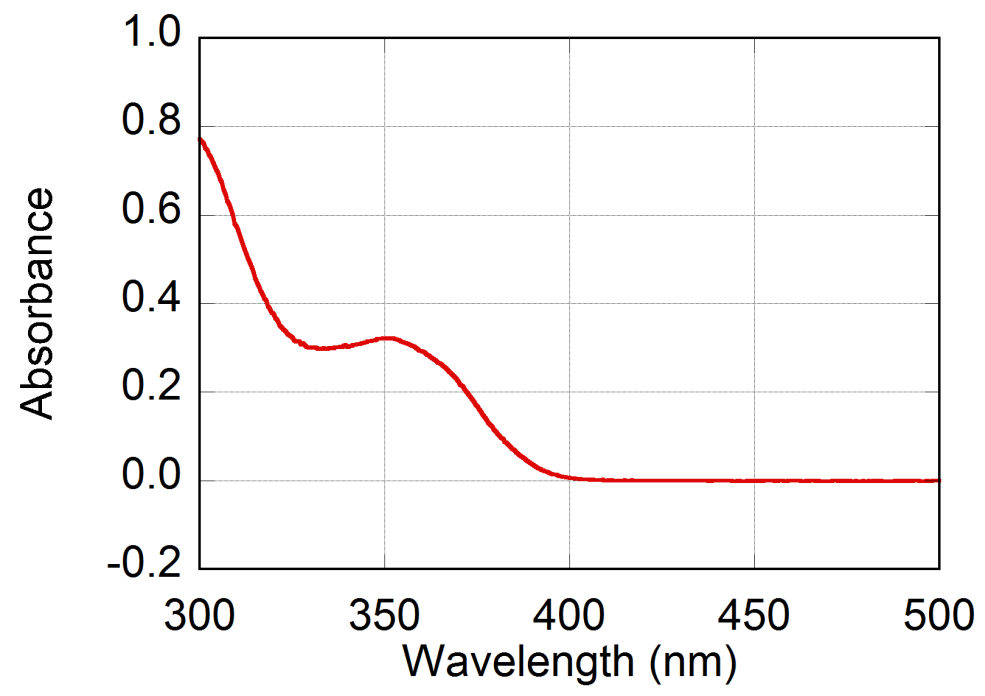

Figure S183. UV-vis absorption spectrum of $\mathbf{3 d}(\mathbf{8 0} / \mathbf{2 0})$ in $\mathrm{PhCF}_{3}\left(1.75 \times 10^{-2} \mathrm{~g} / \mathrm{L}\right.$, light path length $=10 \mathrm{~mm}$ ).

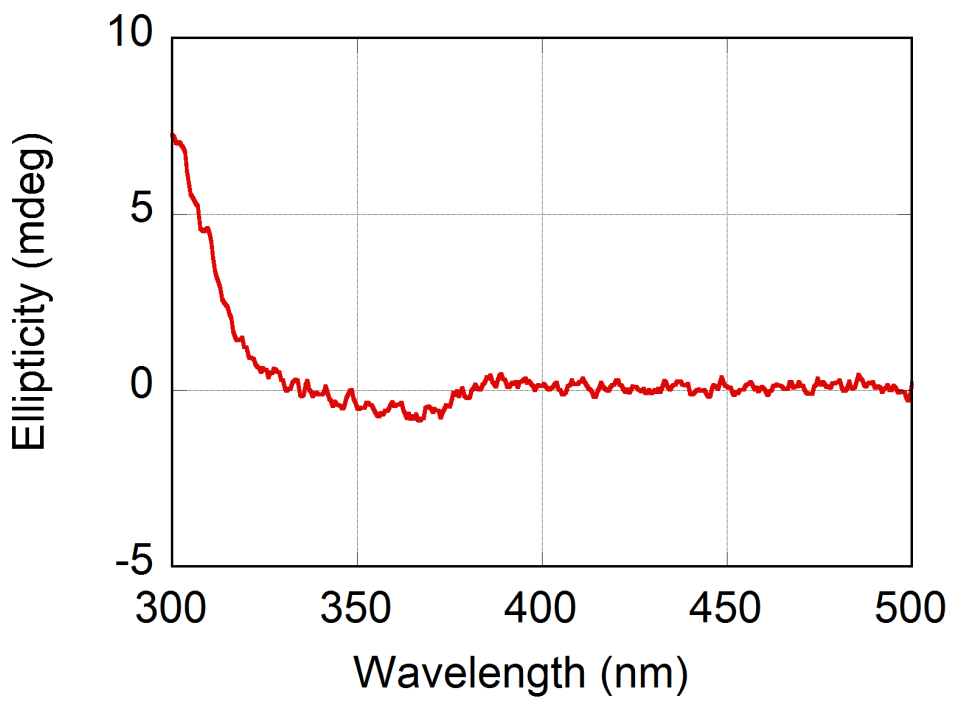

Figure S184. CD spectrum of 3d(80/20) in $\mathrm{PhCF}_{3}\left(1.75 \times 10^{-2} \mathrm{~g} / \mathrm{L}\right.$, light path length $=$ $10 \mathrm{~mm}$ ). 


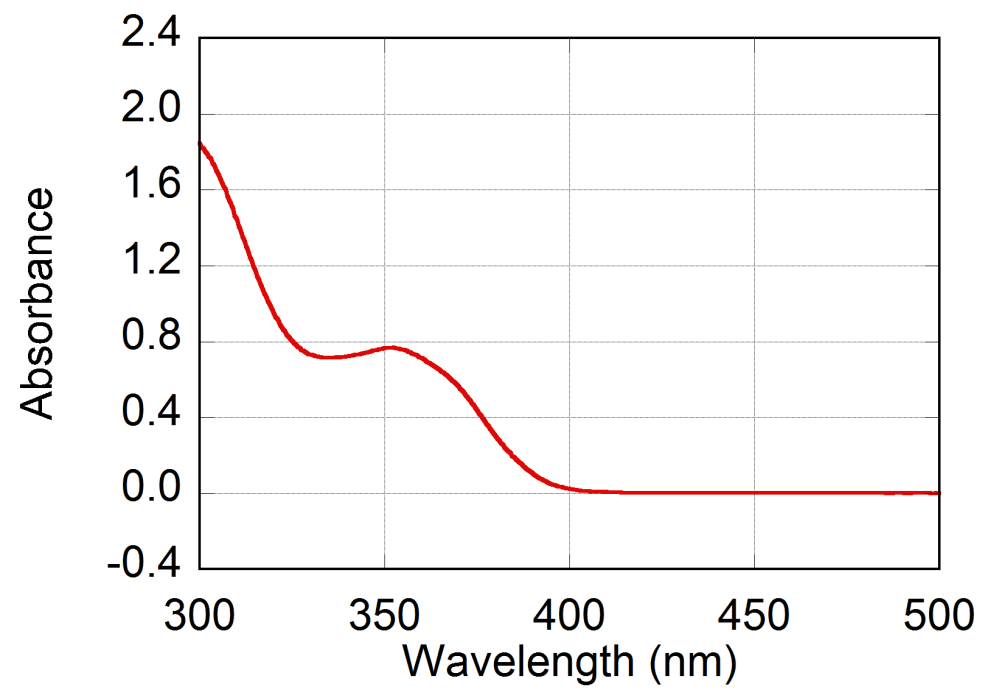

Figure S185. UV-vis absorption spectrum of 3d(30/20) in $\mathrm{PhH}\left(3.75 \times 10^{-2} \mathrm{~g} / \mathrm{L}\right.$, light path length $=10 \mathrm{~mm}$ ).

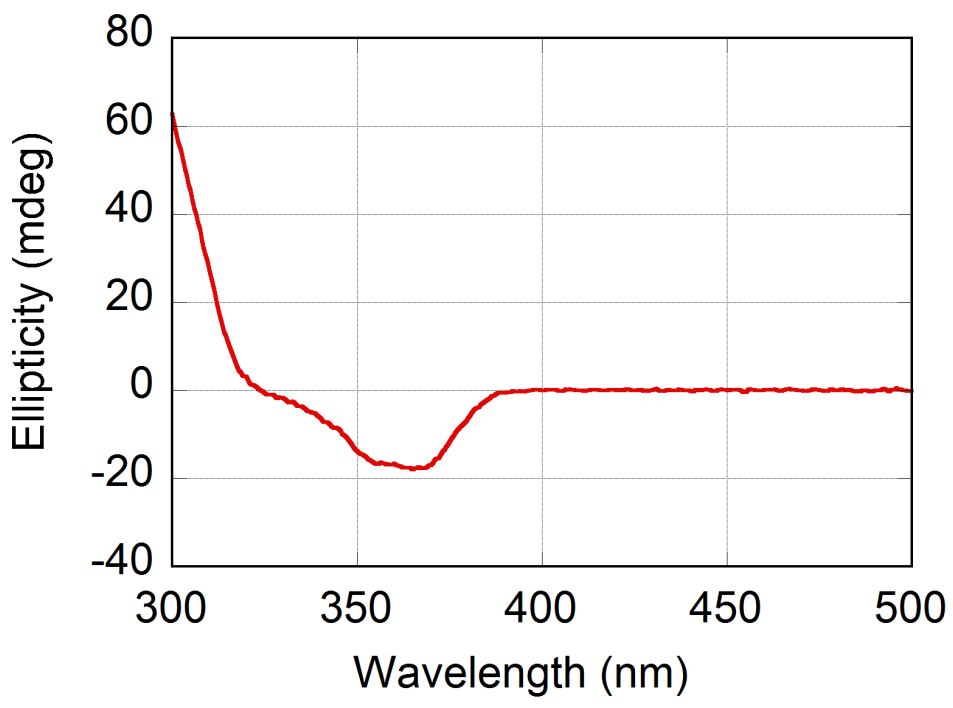

Figure S186. CD spectrum of $\mathbf{3 d}(\mathbf{3 0} / \mathbf{2 0})$ in $\mathrm{PhH}\left(3.75 \times 10^{-2} \mathrm{~g} / \mathrm{L}\right.$, light path length $=10$ $\mathrm{mm})$. 


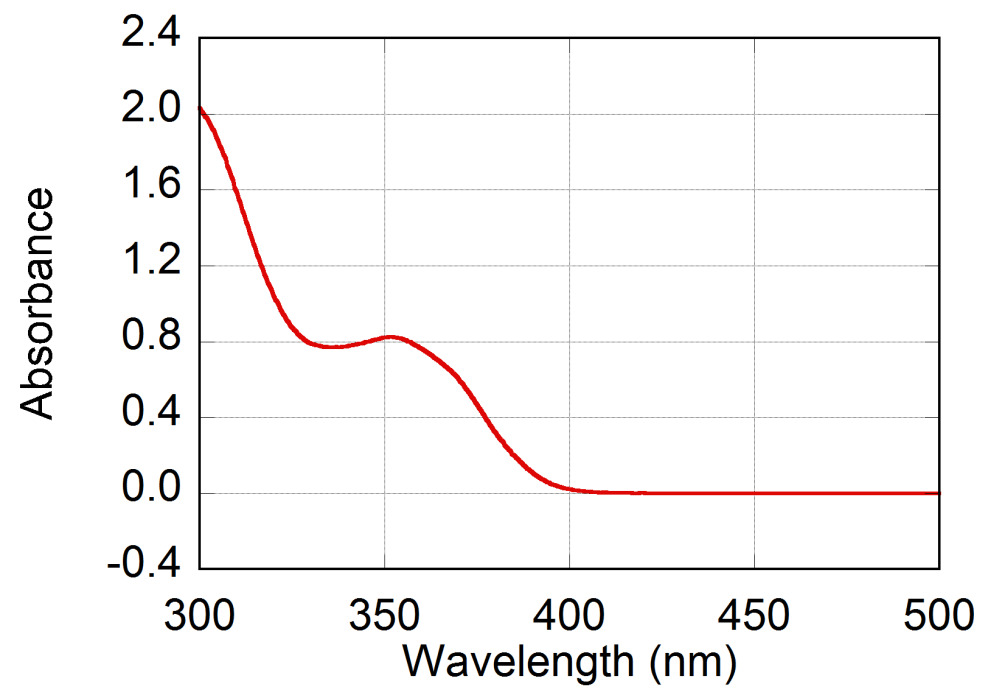

Figure S187. UV-vis absorption spectrum of 3d(60/90) in $\mathrm{PhH}\left(3.62 \times 10^{-2} \mathrm{~g} / \mathrm{L}\right.$, light path length $=10 \mathrm{~mm}$ ).

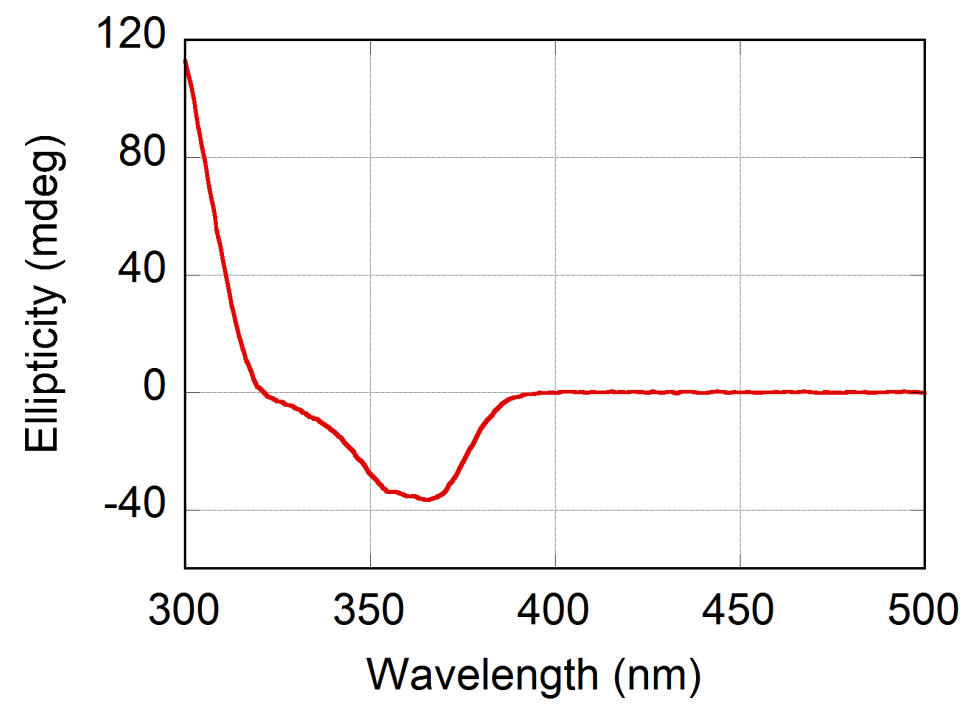

Figure S188. CD spectrum of $\mathbf{3 d}(\mathbf{6 0} / \mathbf{9 0})$ in $\mathrm{PhH}\left(3.62 \times 10^{-2} \mathrm{~g} / \mathrm{L}\right.$, light path length $=10$ $\mathrm{mm})$. 


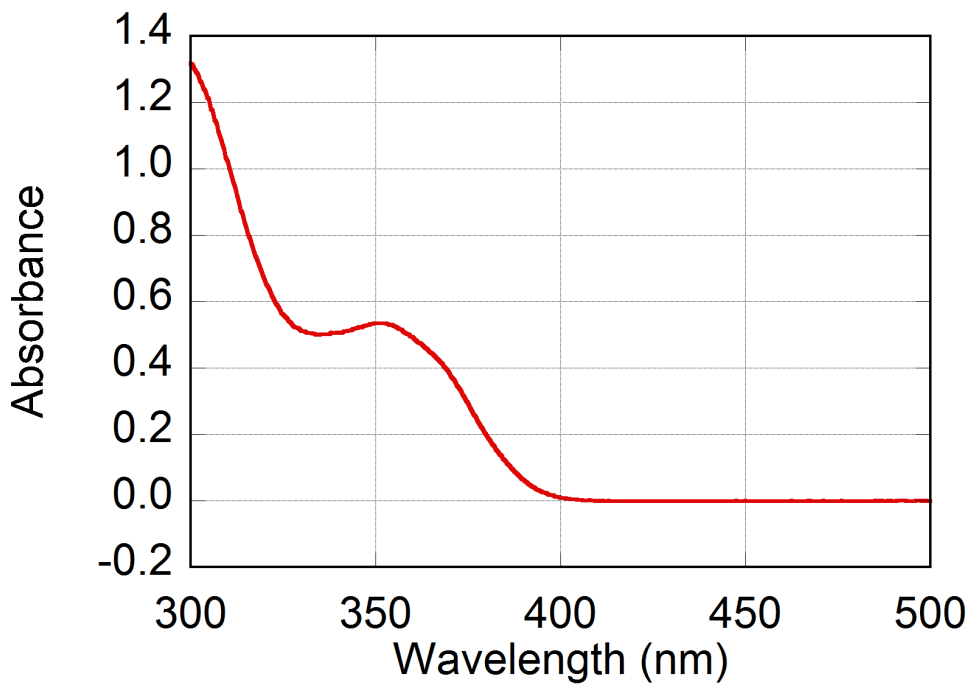

Figure S189. UV-vis absorption spectrum of 3d(80/120) in $\mathrm{PhH}\left(5.90 \times 10^{-2} \mathrm{~g} / \mathrm{L}\right.$, light path length $=10 \mathrm{~mm}$ ).

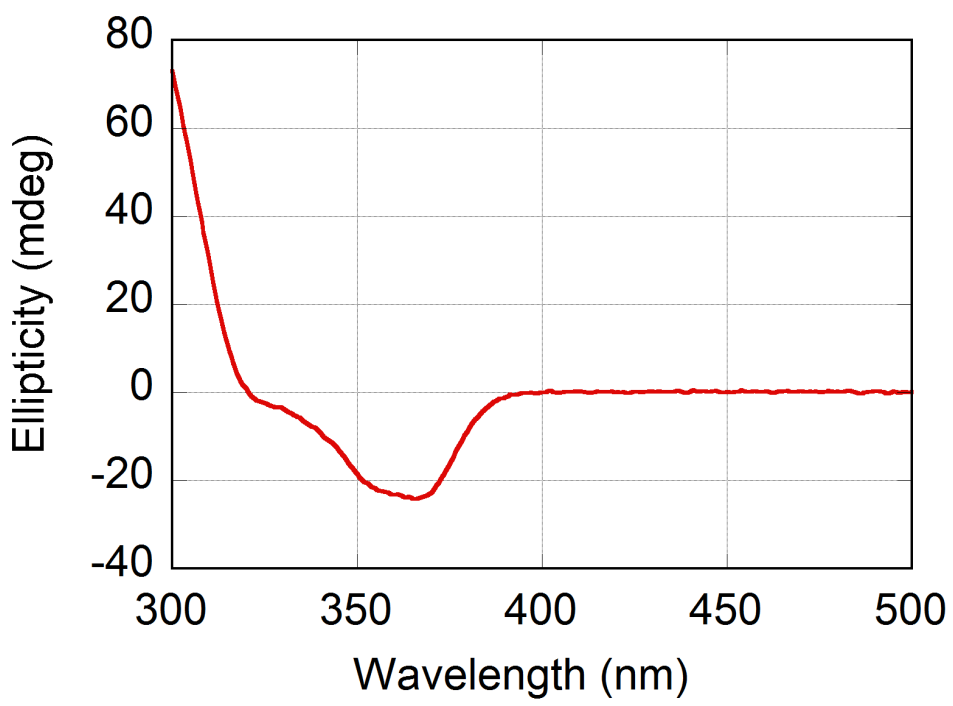

Figure S190. CD spectrum of $\mathbf{3 d}(\mathbf{8 0} / \mathbf{1 2 0})$ in $\mathrm{PhH}\left(5.90 \times 10^{-2} \mathrm{~g} / \mathrm{L}\right.$, light path length $=$ $10 \mathrm{~mm}$ ). 


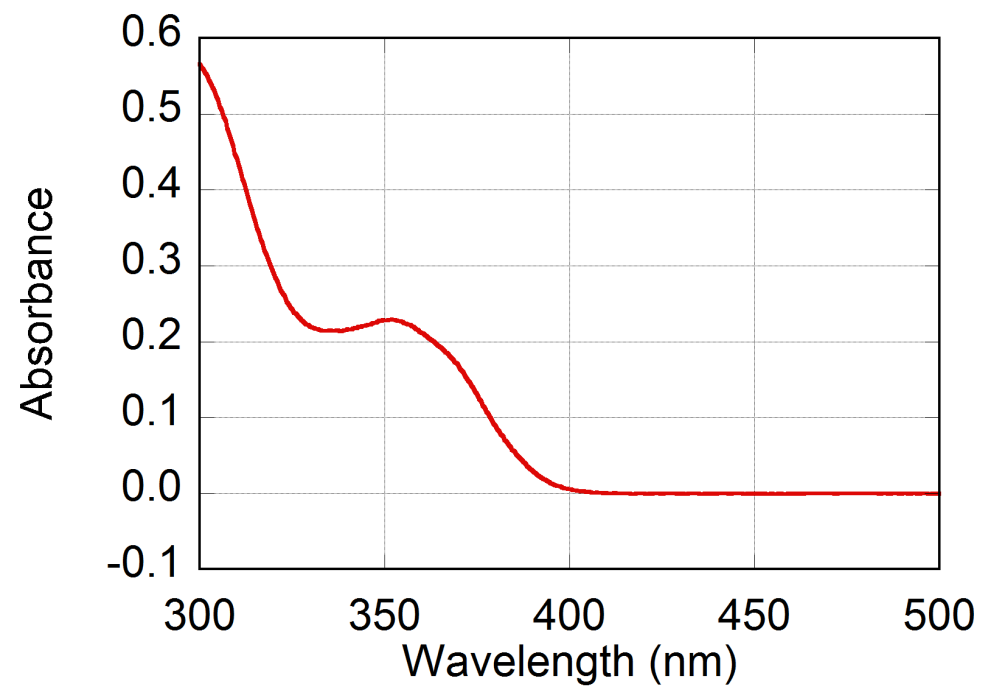

Figure S191. UV-vis absorption spectrum of 3d(100/150) in $\mathrm{PhH}\left(1.39 \times 10^{-2} \mathrm{~g} / \mathrm{L}\right.$, light path length $=10 \mathrm{~mm}$ ).

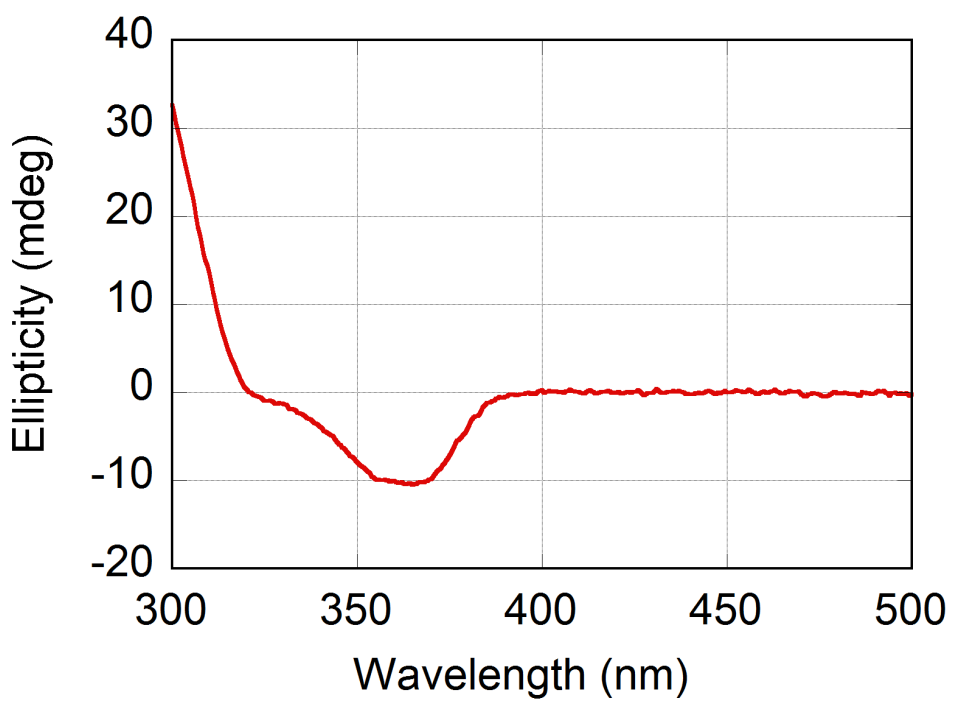

Figure S192. CD spectrum of $\mathbf{3 d}(\mathbf{1 0 0} / \mathbf{1 5 0})$ in $\mathrm{PhH}\left(1.39 \times 10^{-2} \mathrm{~g} / \mathrm{L}\right.$, light path length $=$ $10 \mathrm{~mm}$ ). 


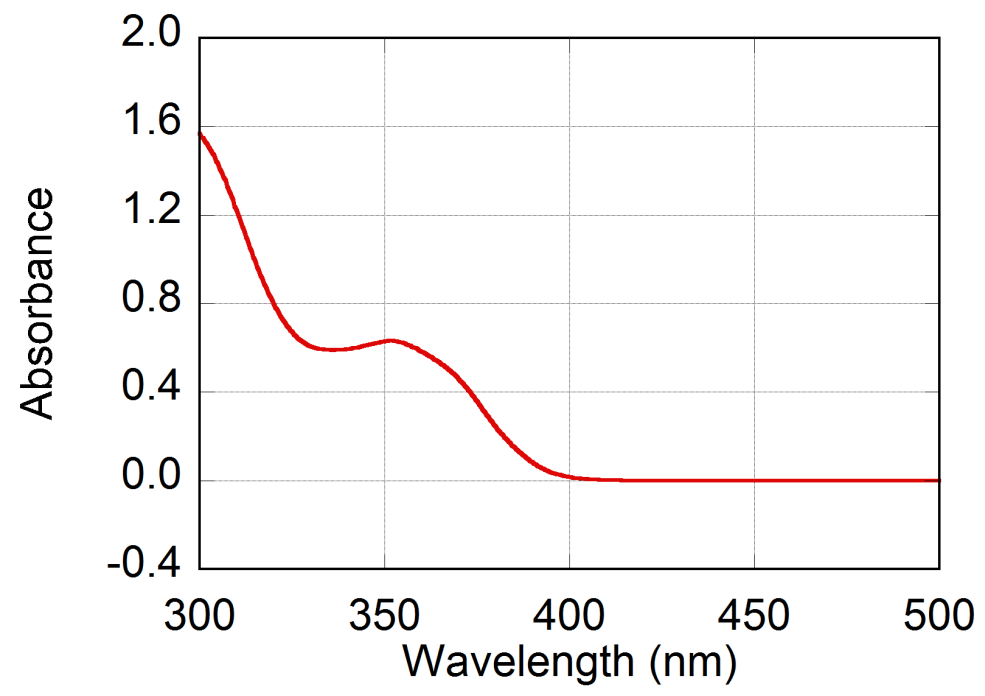

Figure S193. UV-vis absorption spectrum of 3d(120/180) in $\mathrm{PhH}\left(3.07 \times 10^{-2} \mathrm{~g} / \mathrm{L}\right.$, light path length $=10 \mathrm{~mm}$ ).

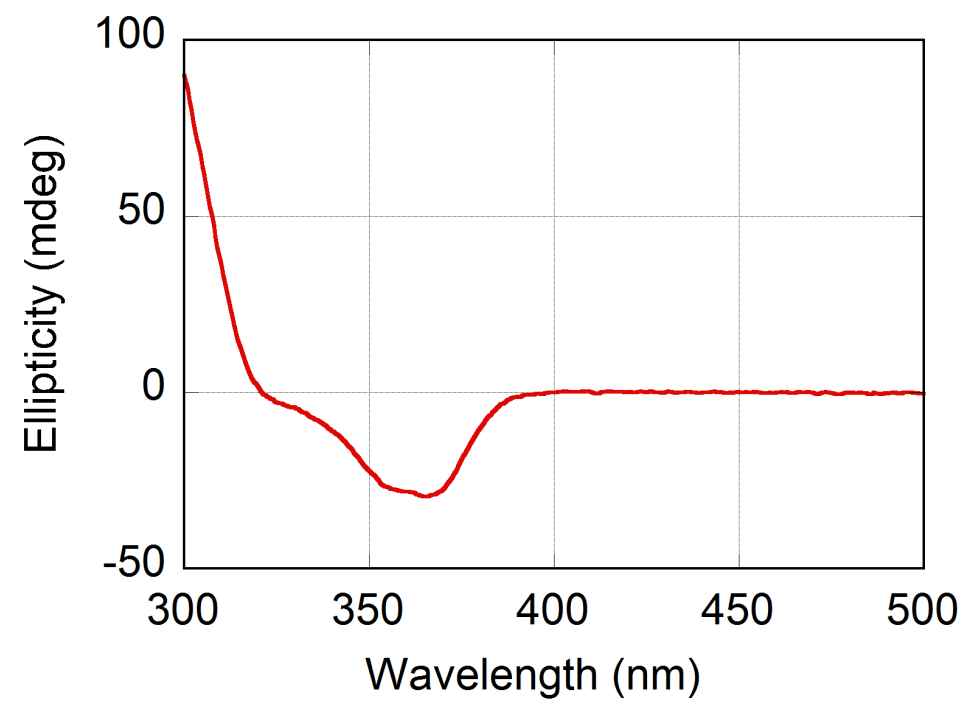

Figure S194. CD spectrum of $\mathbf{3 d}(\mathbf{1 2 0} / \mathbf{1 8 0})$ in $\mathrm{PhH}\left(3.07 \times 10^{-2} \mathrm{~g} / \mathrm{L}\right.$, light path length $=$ $10 \mathrm{~mm}$ ). 


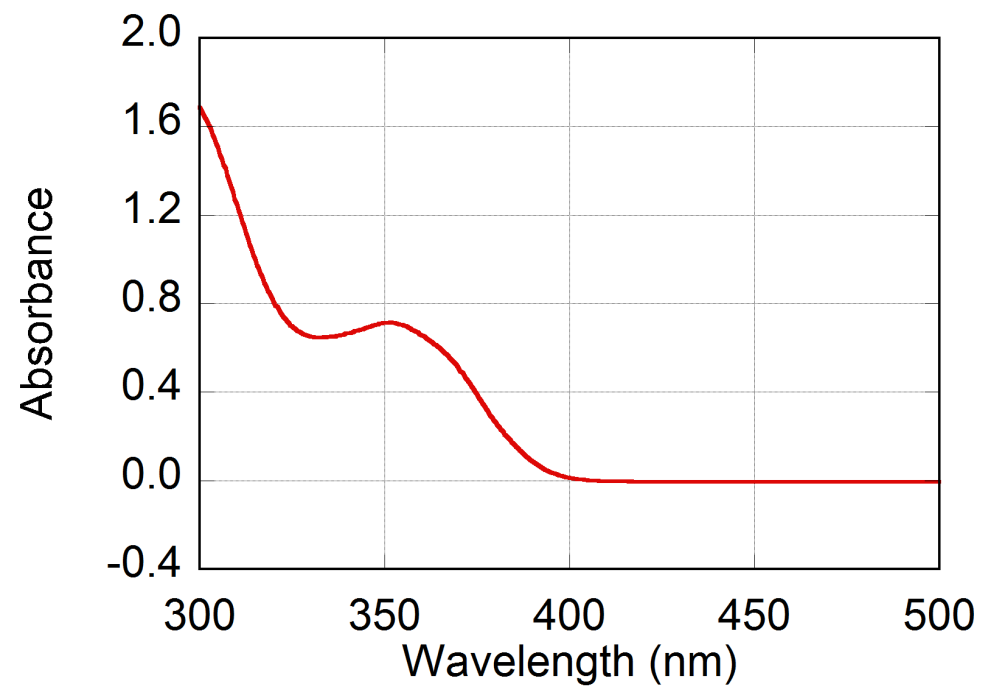

Figure S195. UV-vis absorption spectrum of 3d(20/30) in $\mathrm{PhH}\left(3.75 \times 10^{-2} \mathrm{~g} / \mathrm{L}\right.$, light path length $=10 \mathrm{~mm}$ ).

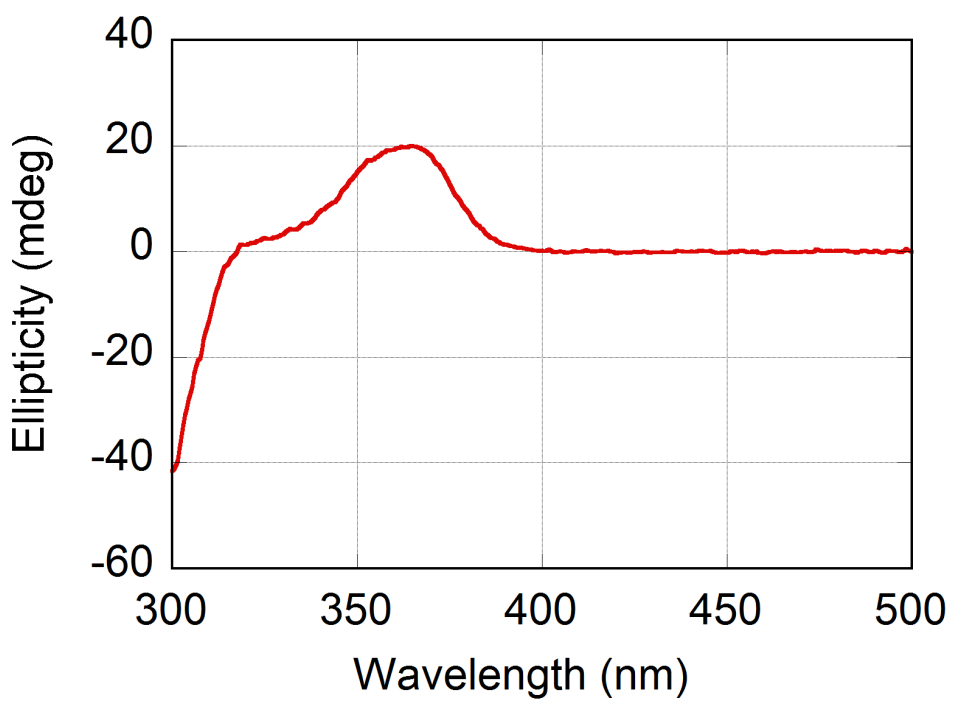

Figure S196. CD spectrum of $\mathbf{3 d}(\mathbf{2 0} / \mathbf{3 0})$ in $\mathrm{PhH}\left(3.75 \times 10^{-2} \mathrm{~g} / \mathrm{L}\right.$, light path length $=10$ $\mathrm{mm})$. 


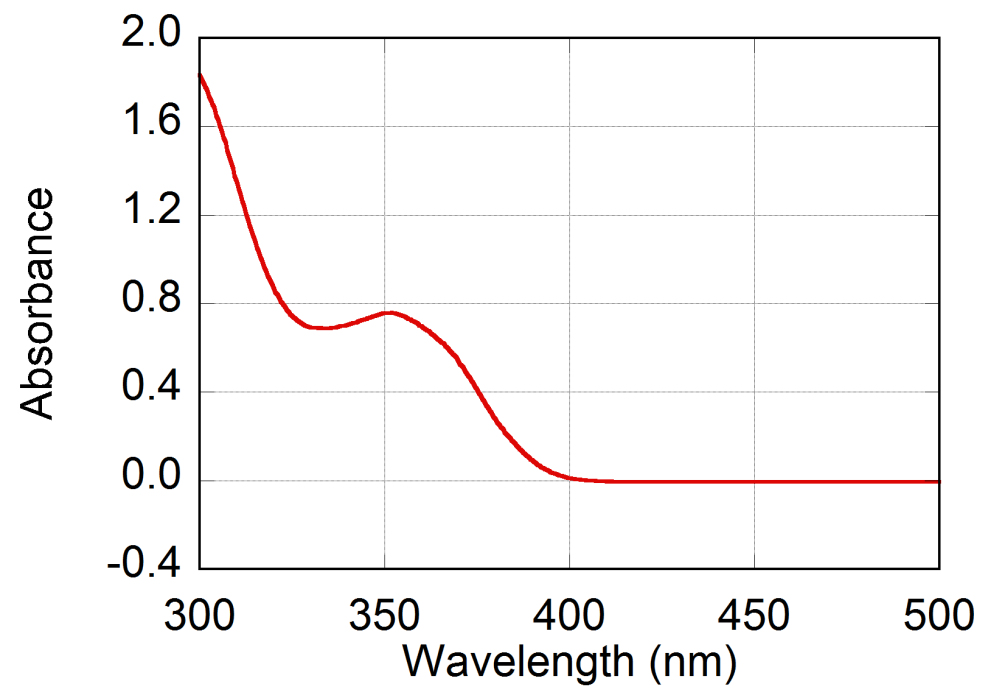

Figure S197. UV-vis absorption spectrum of 3d(60/90) in $\mathrm{PhH}\left(3.62 \times 10^{-2} \mathrm{~g} / \mathrm{L}\right.$, light path length $=10 \mathrm{~mm}$ ).

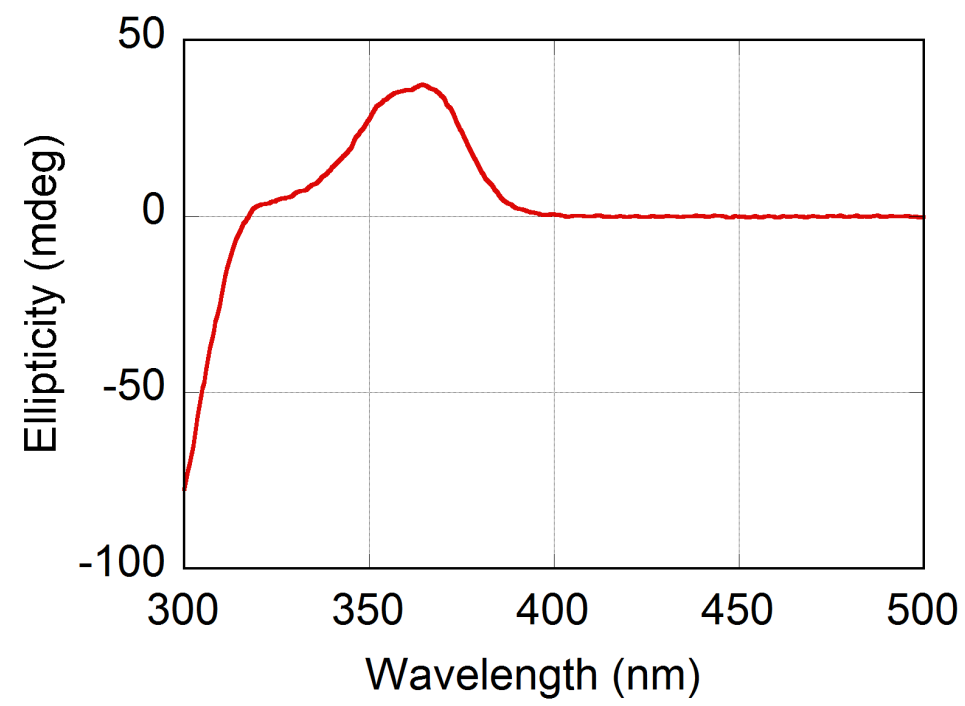

Figure S198. CD spectrum of $\mathbf{3 d}(\mathbf{6 0} / \mathbf{9 0})$ in $\mathrm{PhH}\left(3.62 \times 10^{-2} \mathrm{~g} / \mathrm{L}\right.$, light path length $=10$ $\mathrm{mm})$. 


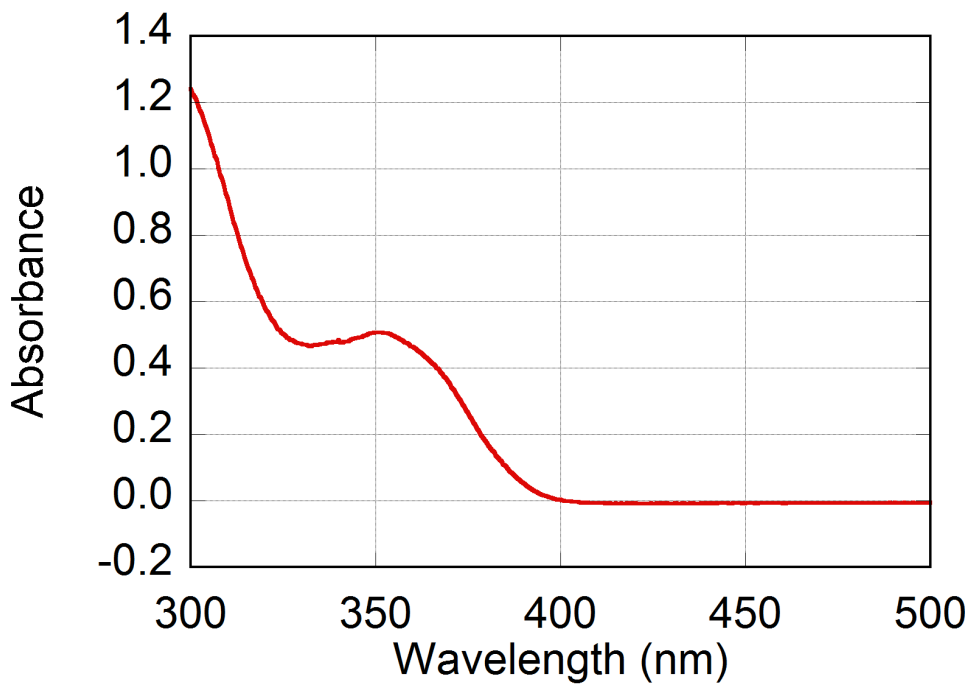

Figure S199. UV-vis absorption spectrum of 3d(80/120) in $\mathrm{PhH}\left(5.90 \times 10^{-2} \mathrm{~g} / \mathrm{L}\right.$, light path length $=10 \mathrm{~mm}$ ).

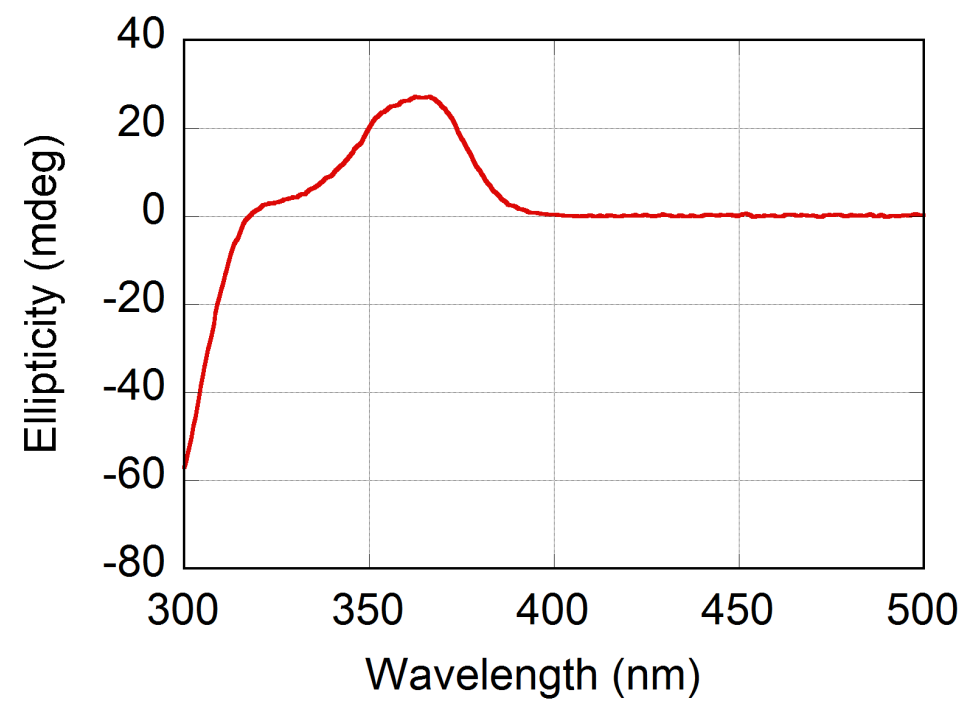

Figure S200. CD spectrum of $\mathbf{3 d}(\mathbf{8 0} / \mathbf{1 2 0})$ in $\mathrm{PhH}\left(5.90 \times 10^{-2} \mathrm{~g} / \mathrm{L}\right.$, light path length $=$ $10 \mathrm{~mm}$ ). 


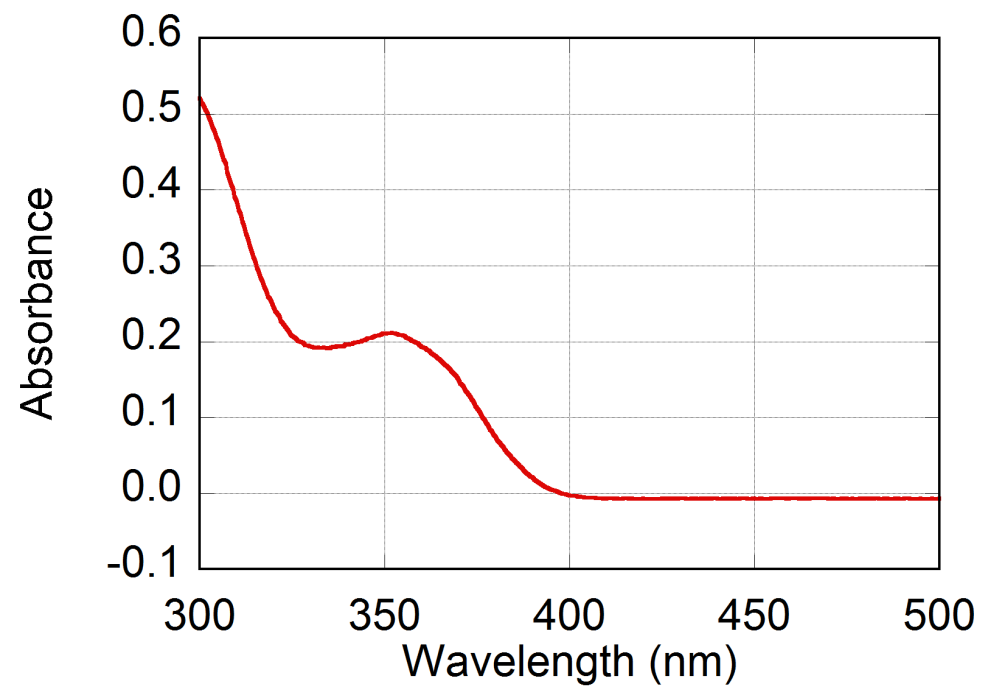

Figure S201. UV-vis absorption spectrum of 3d(100/150) in $\mathrm{PhH}\left(1.39 \times 10^{-2} \mathrm{~g} / \mathrm{L}\right.$, light path length $=10 \mathrm{~mm}$ ).

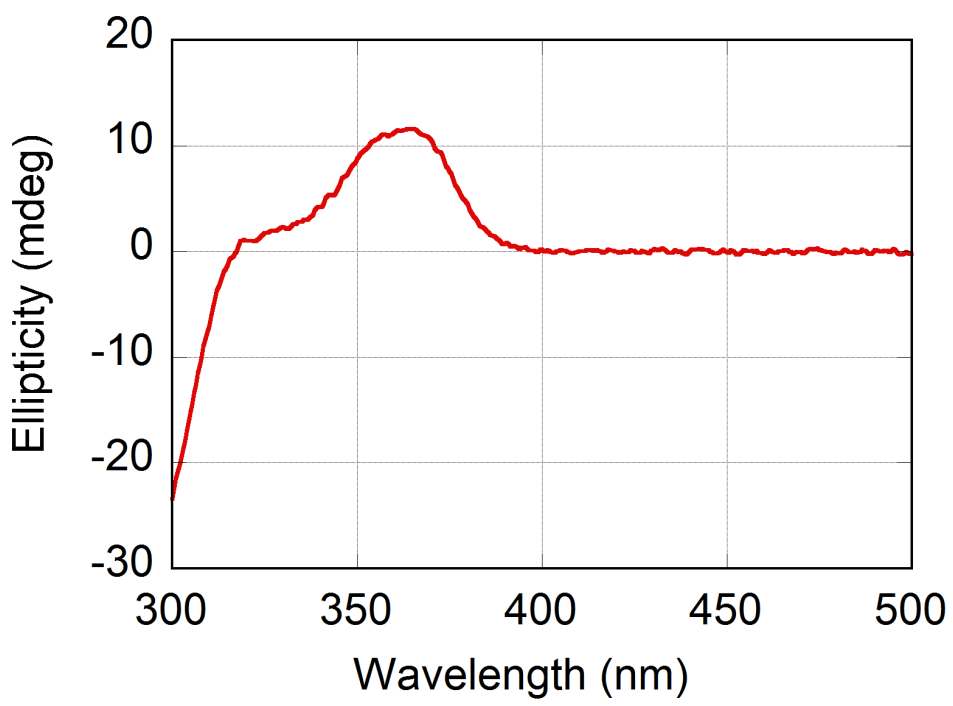

Figure S202. CD spectrum of $\mathbf{3 d}(\mathbf{1 0 0} / \mathbf{1 5 0})$ in $\mathrm{PhH}\left(1.39 \times 10^{-2} \mathrm{~g} / \mathrm{L}\right.$, light path length $=$ $10 \mathrm{~mm}$ ). 


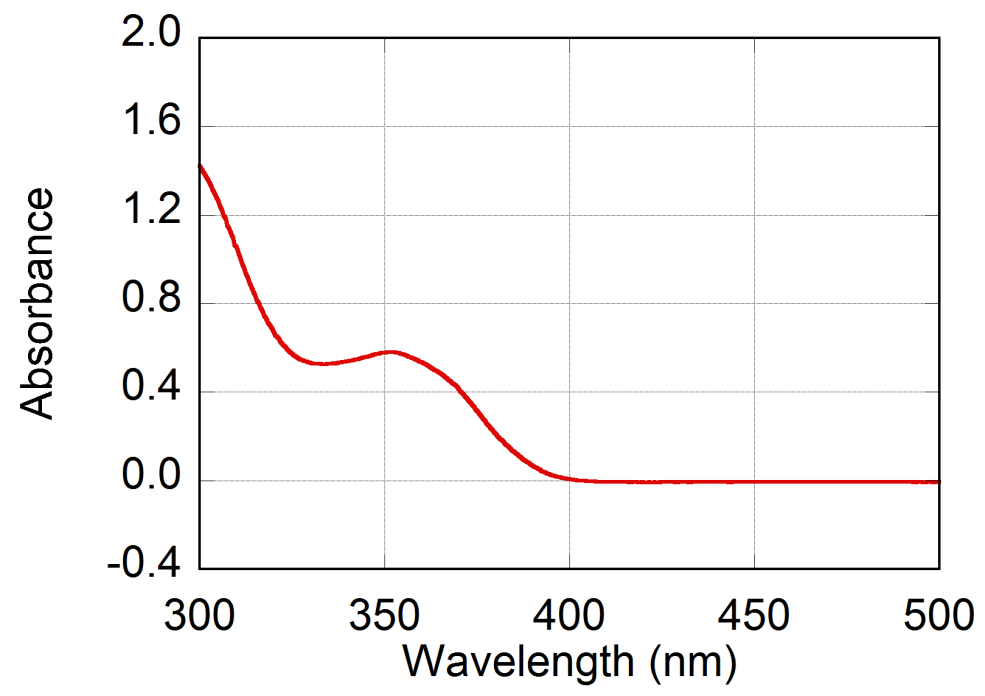

Figure S203. UV-vis absorption spectrum of 3d(120/180) in $\mathrm{PhH}\left(3.07 \times 10^{-2} \mathrm{~g} / \mathrm{L}\right.$, light path length $=10 \mathrm{~mm}$ ).

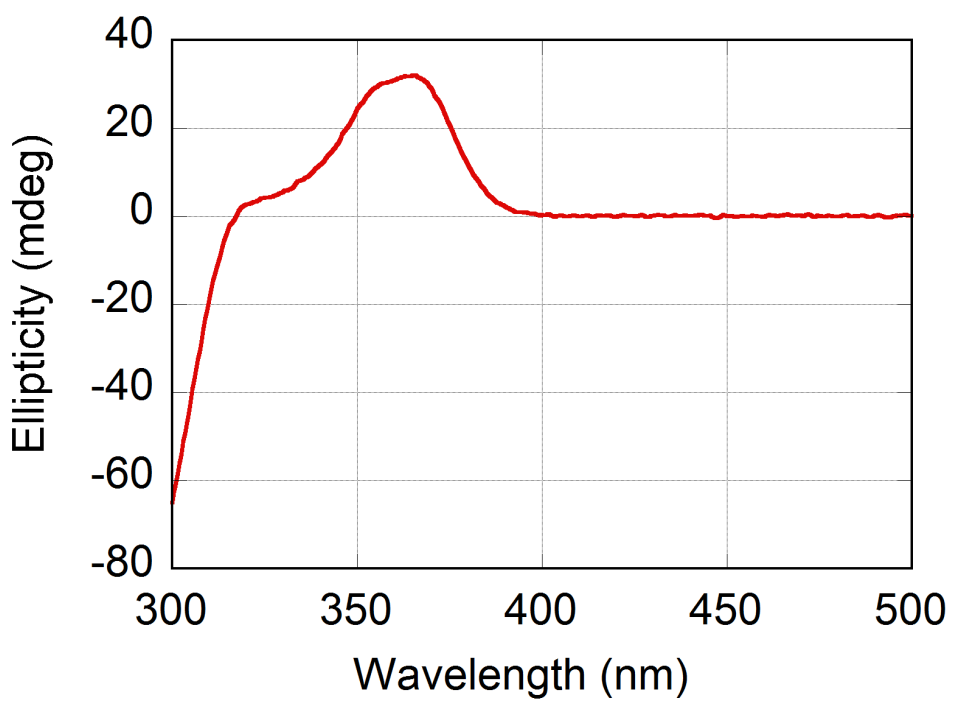

Figure S204. CD spectrum of $\mathbf{3 d}(\mathbf{1 2 0} / \mathbf{1 8 0})$ in $\mathrm{PhH}\left(1.39 \times 10^{-2} \mathrm{~g} / \mathrm{L}\right.$, light path length $=$ $10 \mathrm{~mm}$ ). 


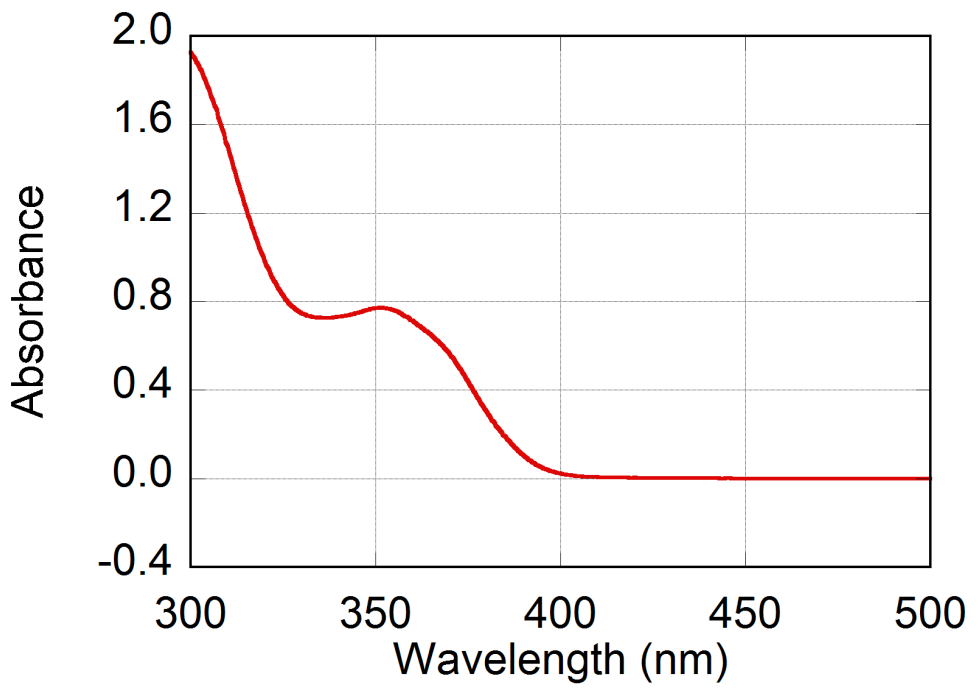

Figure S205. UV-vis absorption spectrum of 4 in $\mathrm{PhH}\left(4.14 \times 10^{-2} \mathrm{~g} / \mathrm{L}\right.$, light path length $=10 \mathrm{~mm}$ ).

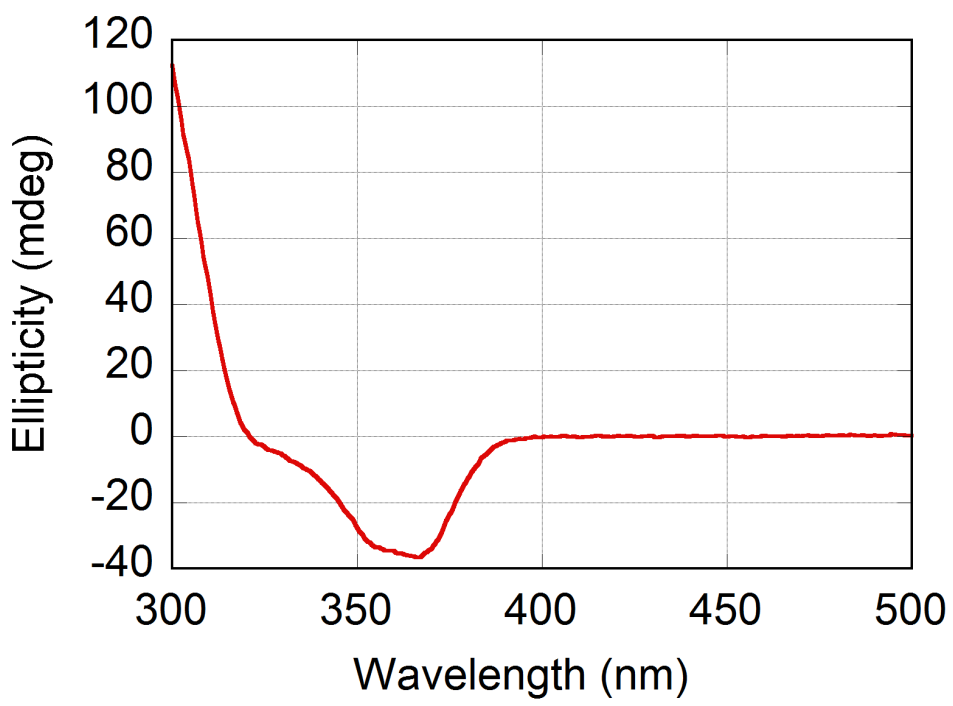

Figure S206. CD spectrum of 4 in $\mathrm{PhH}\left(4.14 \times 10^{-2} \mathrm{~g} / \mathrm{L}\right.$, light path length $\left.=10 \mathrm{~mm}\right)$. 


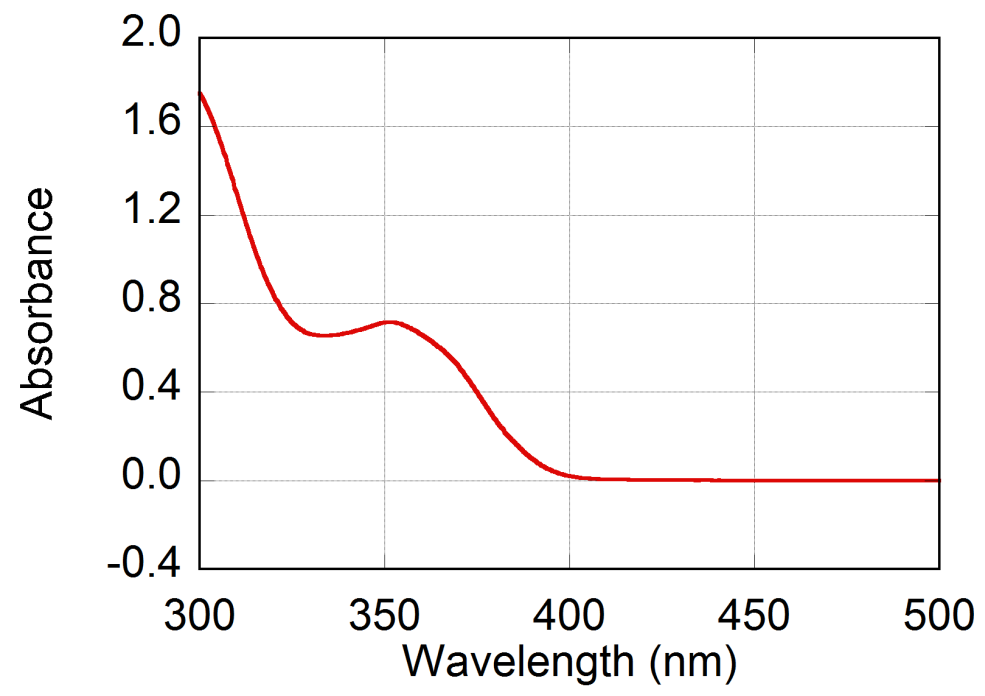

Figure S207. UV-vis absorption spectrum of 4 in $\mathrm{PhCF}_{3}\left(4.14 \times 10^{-2} \mathrm{~g} / \mathrm{L}\right.$, light path length $=10 \mathrm{~mm})$.

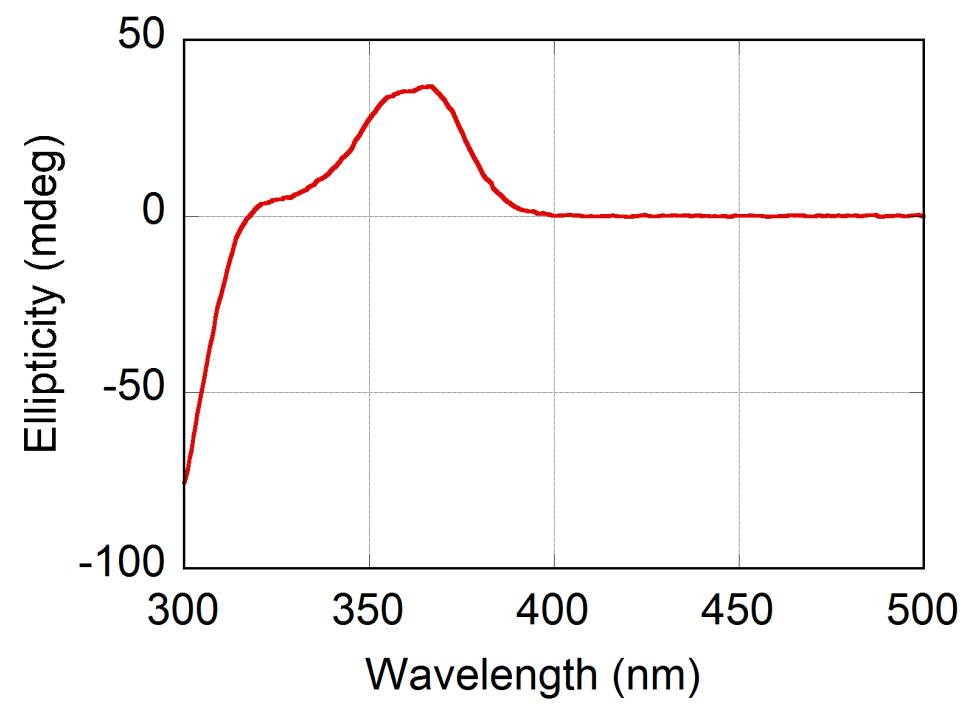

Figure S208. CD spectrum of 4 in $\mathrm{PhCF}_{3}\left(4.14 \times 10^{-2} \mathrm{~g} / \mathrm{L}\right.$, light path length $\left.=10 \mathrm{~mm}\right)$. 


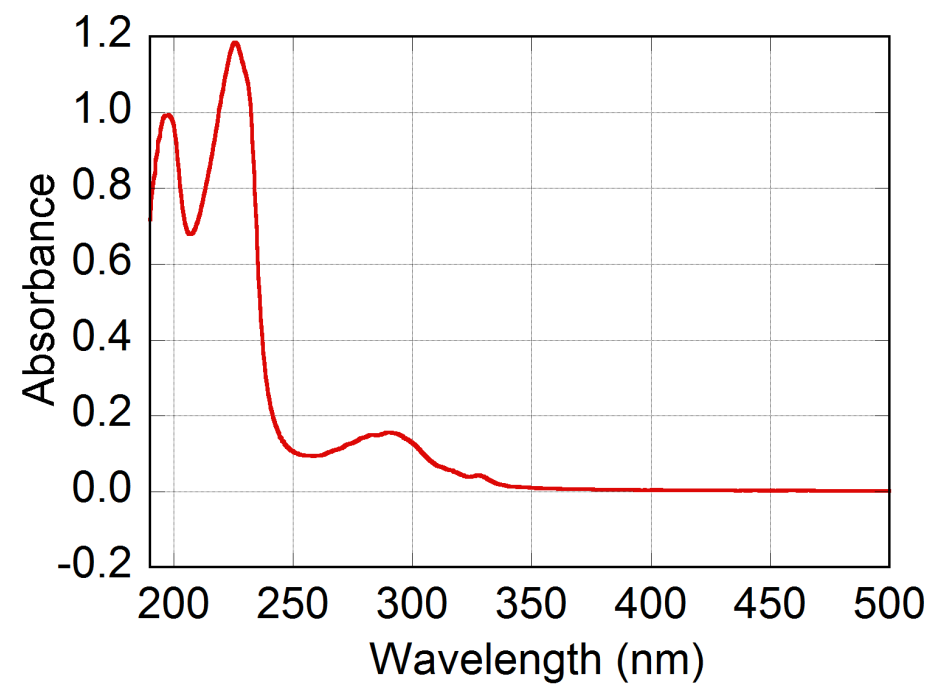

Figure S209. UV-vis absorption spectrum of $(R)-7$ in $\mathrm{MeCN}\left(2.25 \times 10^{-4} \mathrm{~mol} / \mathrm{L}\right.$, light path length $=10 \mathrm{~mm}$ ). This compound 7 was obtained by the asymmetric Suzuki-Miyaura cross-coupling reaction using polymer $\mathbf{4}$ as a ligand in $\mathrm{PhH}$.

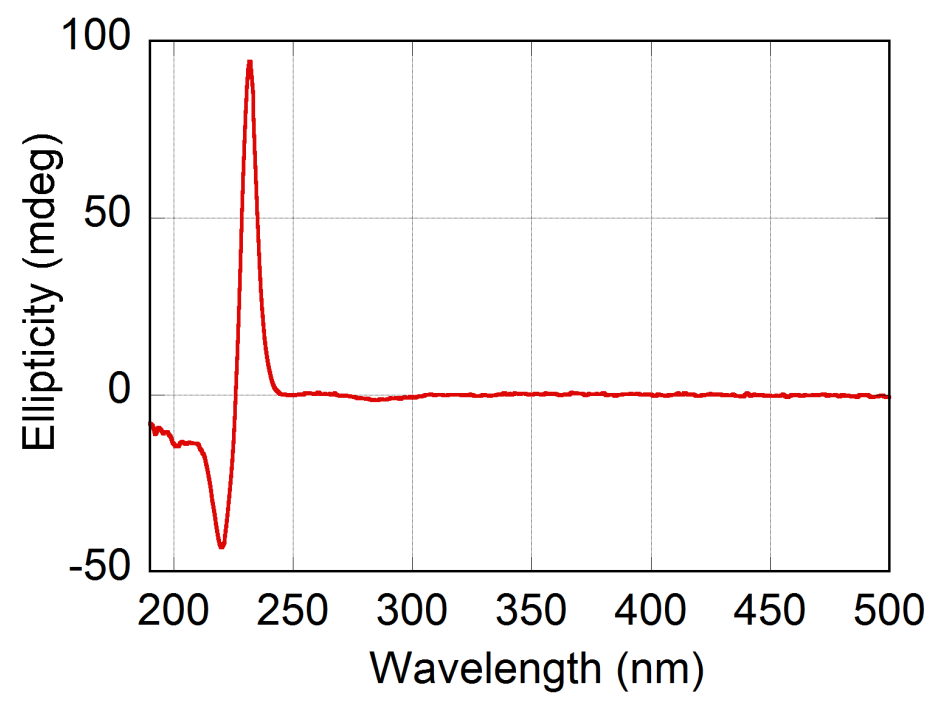

Figure S210. CD spectrum of $(R)-7$ in $\mathrm{MeCN}\left(2.25 \times 10^{-4} \mathrm{~mol} / \mathrm{L}\right.$, light path length $=10$ $\mathrm{mm}$ ). This compound 7 was obtained by the asymmetric Suzuki-Miyaura cross-coupling reaction using polymer $\mathbf{4}$ as a ligand in $\mathrm{PhH}$. 


\section{Theoretical Calculation of ECD spectrum of 7}

In order to determine the absolute configuration of 7, theoretical calculations of $(R)-7$ was carried out using the Gaussian09 suite of programs. ${ }^{12}$ The geometry optimization and the time-dependent DFT calculations were performed at the $\omega \mathrm{B} 97 \mathrm{XD} / 6-31 \mathrm{~g}(\mathrm{~d}, \mathrm{p})$ level. The obtained ECD spectrum was in good agreement with the CD spectrum of 7, which was obtained by the asymmetric Suzuki-Miyaura cross-coupling reaction using polymer 4 as a ligand in $\mathrm{PhH}$. Therefore we concluded that $(R)-7$ or $(S)-7$ were obtained in $\mathrm{PhH}$ or $\mathrm{PhCF}_{3}$, respectively. These results are also good agreement with our previous report. $^{13}$

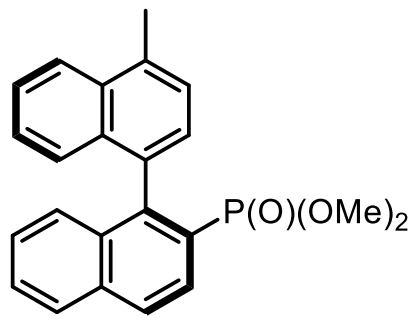

$(R)-7$

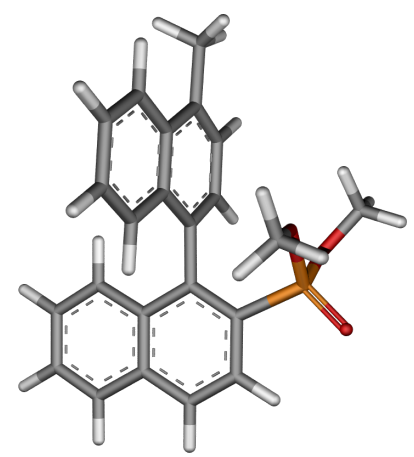

Figure S211. Molecular structure (left) and geometry optimized structure (right) of $(R)-7$.

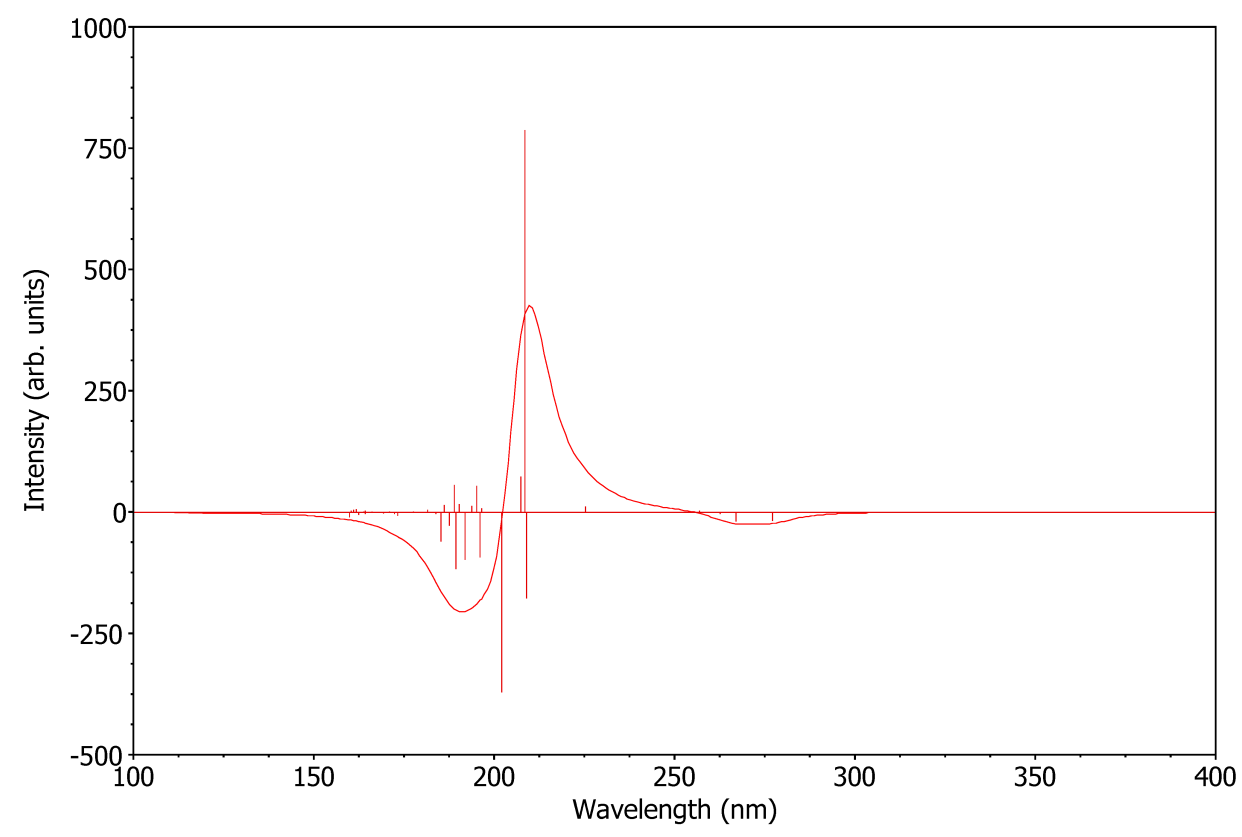

Figure S212. Calculated ECD spectrum of $(R)-7$. 


\section{CD spectra of 3(120/180) and 4 in $\mathrm{PhH}$ or $\mathrm{PhCF}_{3}$}

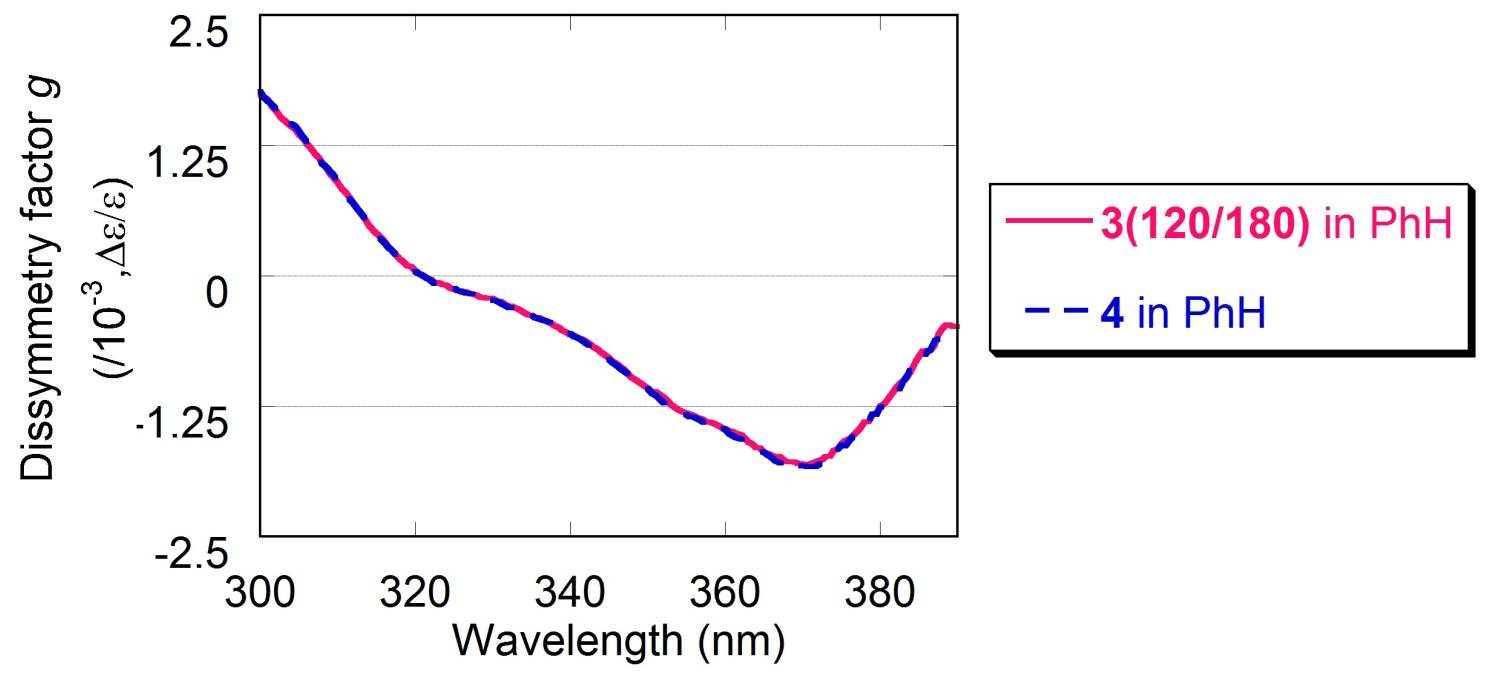

Figure S213. CD spectra of 3(120/180) and 4 in $\mathrm{PhH}$.

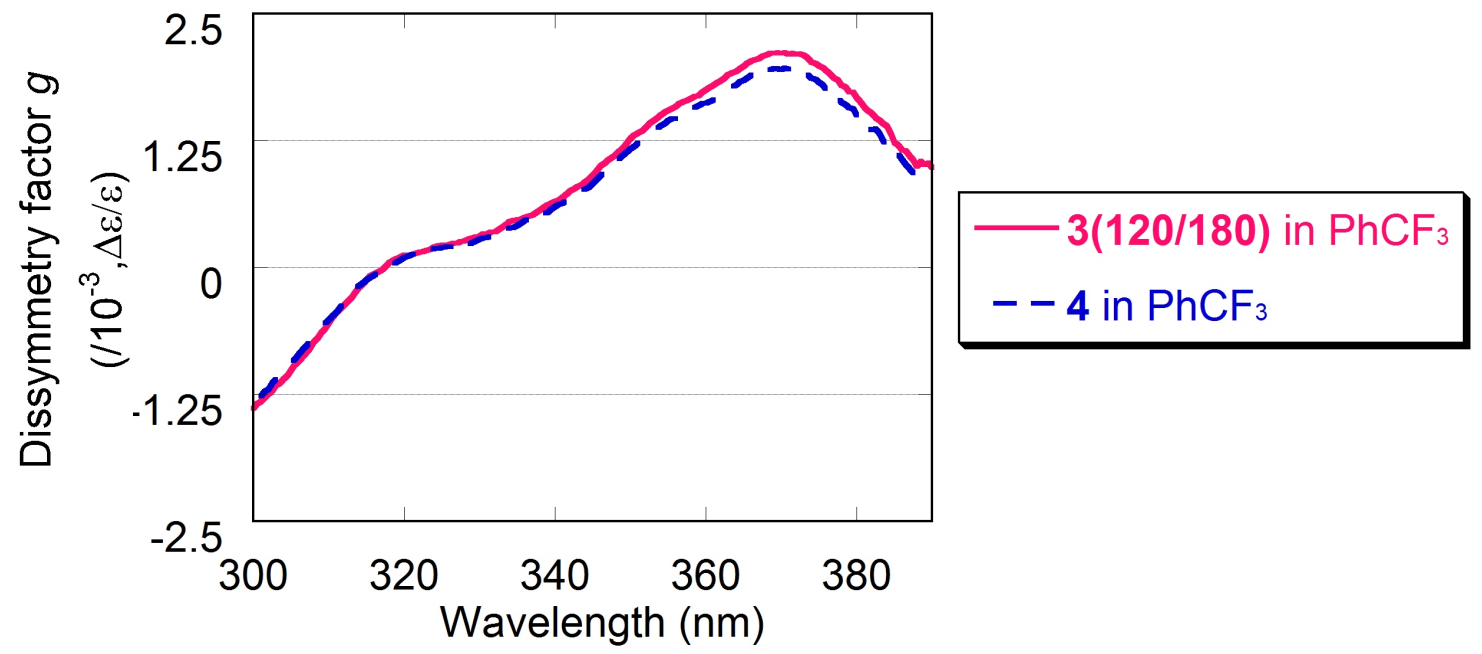

Figure S214. CD spectra of 3(120/180) and 4 in $\mathrm{PhCF}_{3}$. 


\section{$8 \quad$ References}

(1) Carmona, E.; Paneque, M.; Poveda, M. L. Polyhedron 1989, 8, 285-291.

(2) Yamada, T.; Nagata, Y.; Suginome, M. Chem. Commun. 2010, 46, 4914-4916.

(3) Nagata, Y.; Nishikawa, T.; Suginome, M. Chem. Commun. 2012, 48, 11193-11195.

(4) Nagata, Y.; Yamada, T.; Adachi, T.; Akai, Y.; Yamamoto, T.; Suginome, M. J. Am. Chem. Soc. 2013, 135, 10104-10113.

(5) Nagata, Y.; Suginome, M. J. Polym. Sci., Part A: Polym. Chem. 2011, 49, 4275-4282.

(6) Ito, Y.; Ihara, E.; Uesaka, T.; Murakami, M. Macromolecules 1992, 25, 6711-6713.

(7) Yamamoto, T.; Yamada, T.; Nagata, Y.; Suginome, M. J. Am. Chem. Soc. 2010, 132, 7899-7901.

(8) Nagata, Y.; Nishikawa, T.; Suginome, M. J. Am. Chem. Soc. 2014, 136, 15901-15904.

(9) Nagata, Y.; Nishikawa, T.; Suginome, M. J. Am. Chem. Soc. 2015, 137, 4070-4073.

(10) X. Shen, G. O. Jones, D. A. Watson, B. Bhayana, S. L. Buchwald, J. Am. Chem. Soc. 2010, 132, 11278-11287.

(11) 7 could be synthesized by another method (98\% ee $(R)$ ), Yamamoto, T.; Akai, Y.; Suginome, M. to be submitted.

(12) Gaussian 09, Revision D.01, Frisch, M. J.; Trucks, G. W.; Schlegel, H. B.; Scuseria, G. E.; Robb, M. A.; Cheeseman, J. R.; Scalmani, G.; Barone, V.; Mennucci, B.; Petersson, G. A.; Nakatsuji, H.; Caricato, M.; Li, X.; Hratchian, H. P.; Izmaylov, A. F.; Bloino, J.; Zheng, G.; Sonnenberg, J. L.; Hada, M.; Ehara, M.; Toyota, K.; Fukuda, R.; Hasegawa, J.; Ishida, M.; Nakajima, T.; Honda, Y.; Kitao, O.; Nakai, H.; Vreven, T.; Montgomery, J. A., Jr.; Peralta, J. E.; Ogliaro, F.; Bearpark, M.; Heyd, J. J.; Brothers, E.; Kudin, K. N.; Staroverov, V. N.; Kobayashi, R.; Normand, J.; Raghavachari, K.; Rendell, A.; Burant, J. C.; Iyengar, S. S.; Tomasi, J.; Cossi, M.; Rega, N.; Millam, N. J.; Klene, M.; Knox, J. E.; Cross, J. B.; Bakken, V.; Adamo, C.; Jaramillo, J.; Gomperts, R.; Stratmann, R. E.; Yazyev, O.; Austin, A. J.; Cammi, R.; Pomelli, C.; Ochterski, J. W.; Martin, R. L.; Morokuma, K.; Zakrzewski, V. G.; Voth, G. A.; Salvador, 
P.; Dannenberg, J. J.; Dapprich, S.; Daniels, A. D.; Farkas, Ö.; Foresman, J. B.; Ortiz, J. V.; Cioslowski, J.; Fox, D. J. Gaussian, Inc., Wallingford CT, 2013.

(13) Yamamoto, T.; Akai, Y.; Nagata, Y.; Suginome, M. Angew. Chem., Int. Ed. 2011, 50, 8844-8847. 


\section{NMR spectra of New Compounds}

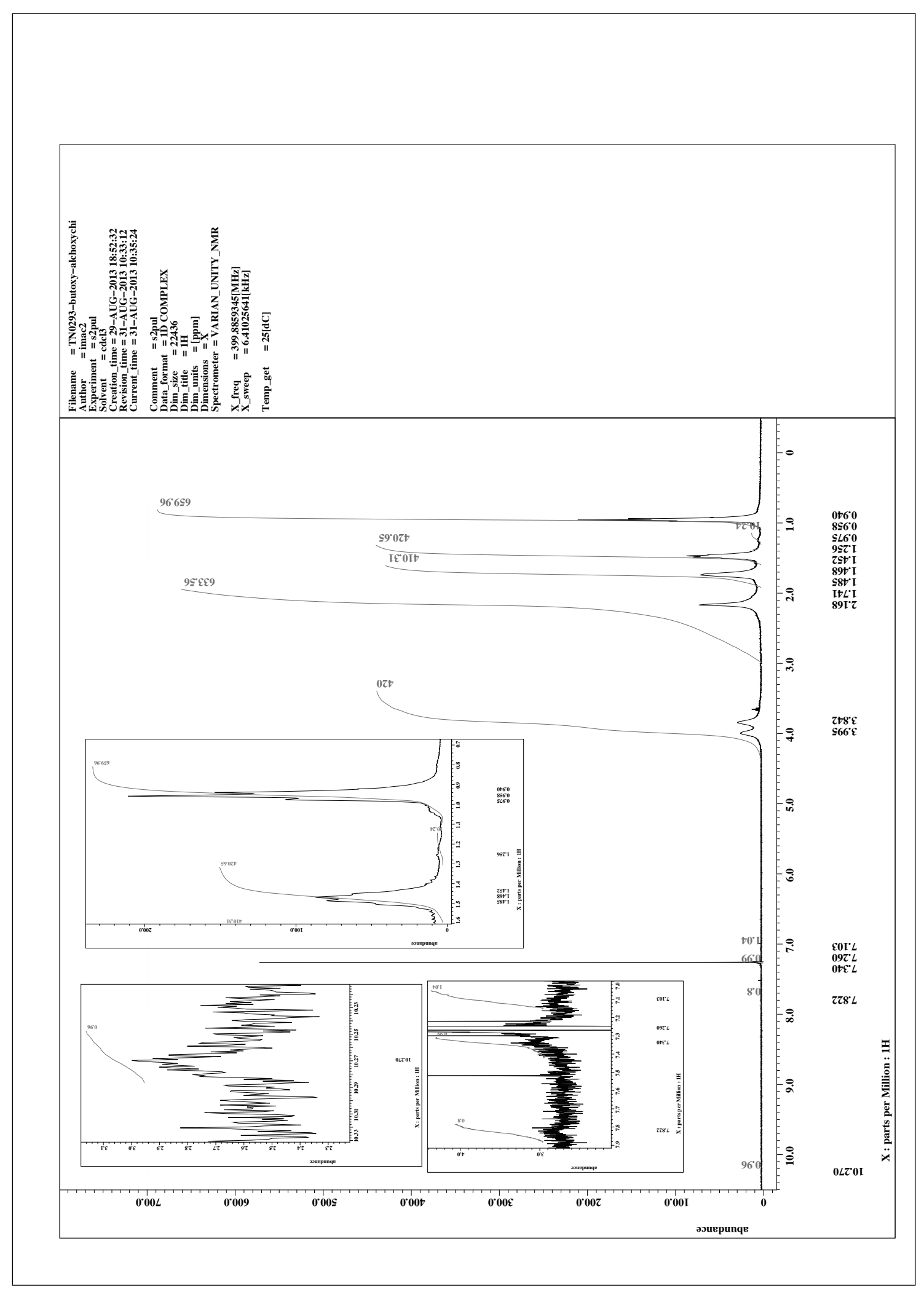

Figure S215. ${ }^{1} \mathrm{H}$ NMR spectrum of $\mathbf{2 b}(\mathbf{5 / 1 0 0})$ in $\mathrm{CDCl}_{3}$. 


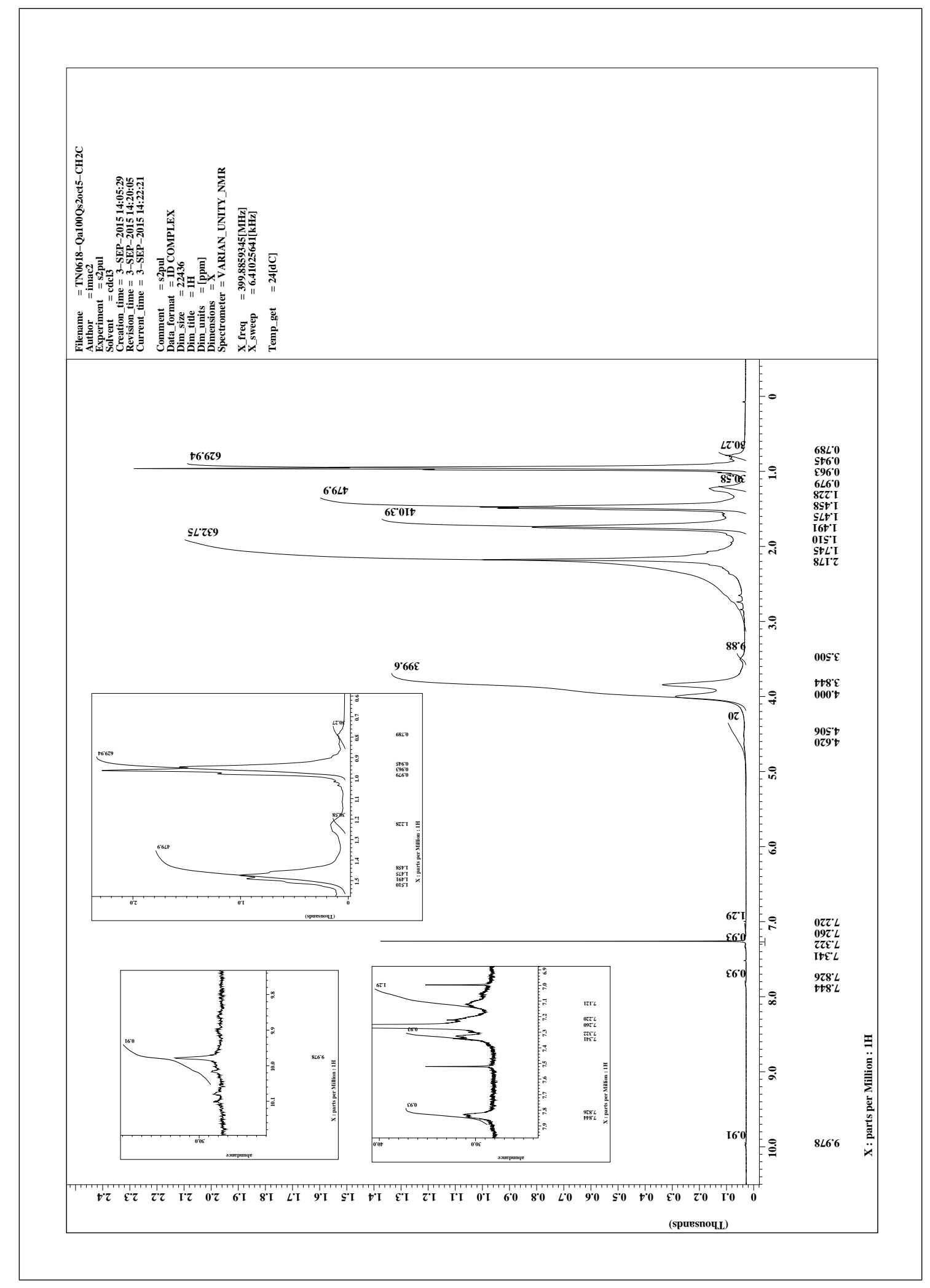

Figure S216. ${ }^{1} \mathrm{H}$ NMR spectrum of $\mathbf{2 c}(\mathbf{5 / 1 0 0})$ in $\mathrm{CDCl}_{3}$. 


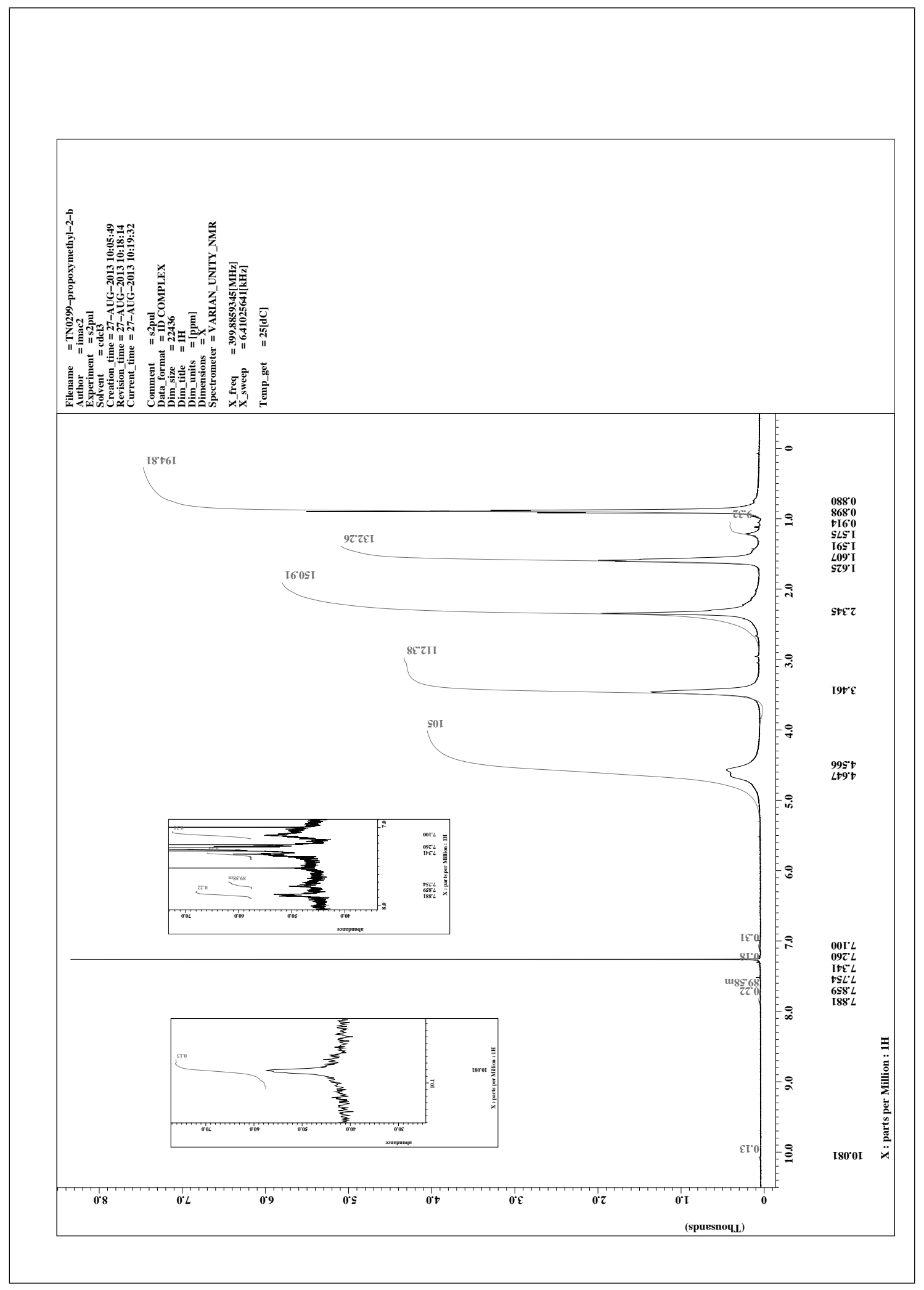

Figure S217. ${ }^{1} \mathrm{H}$ NMR spectrum of $\mathbf{3 b}(\mathbf{5} / \mathbf{1 0 0})$ in $\mathrm{CDCl}_{3}$. 


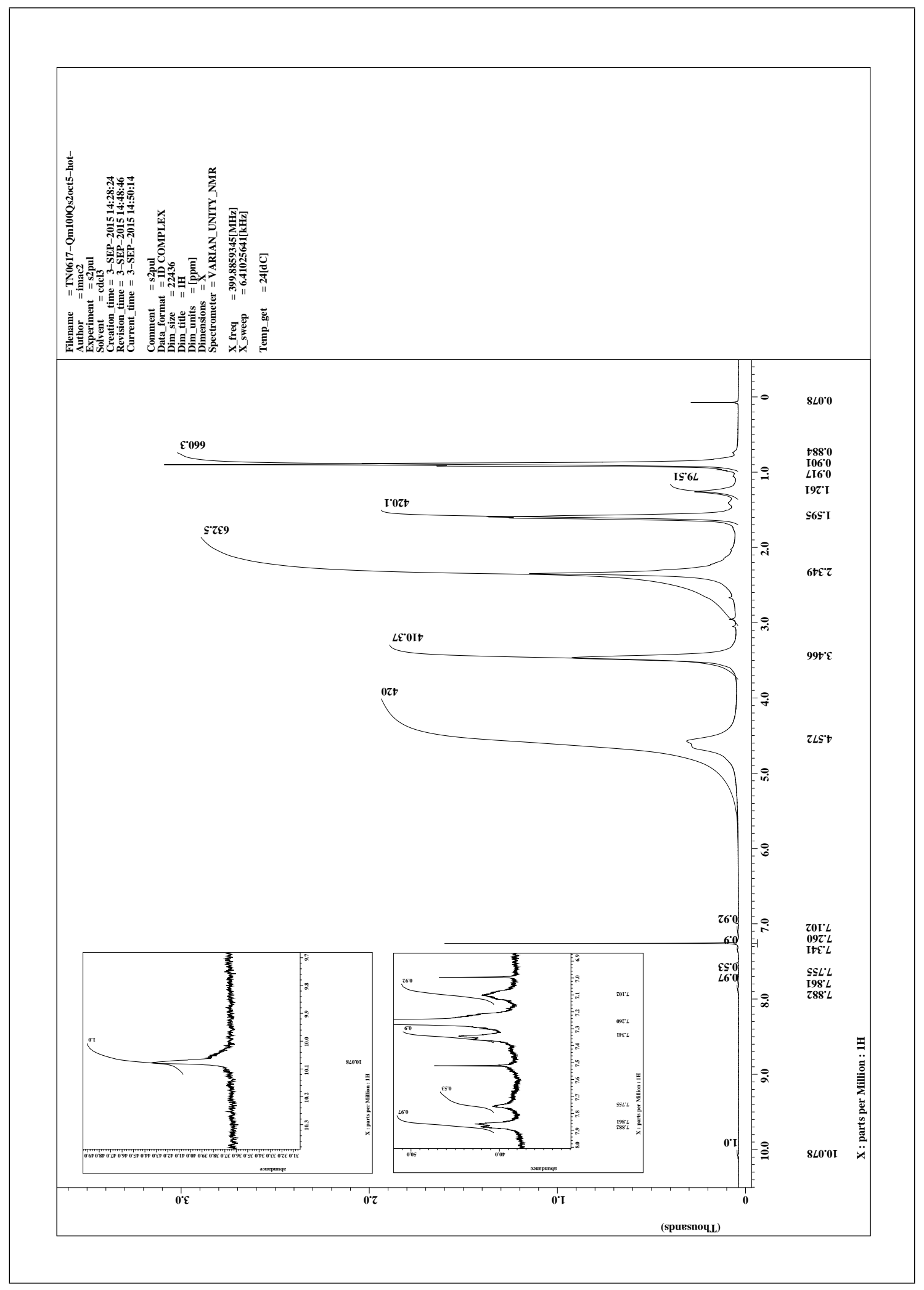

Figure S218. ${ }^{1} \mathrm{H}$ NMR spectrum of $\mathbf{3 c}(\mathbf{5} / \mathbf{1 0 0})$ in $\mathrm{CDCl}_{3}$. 


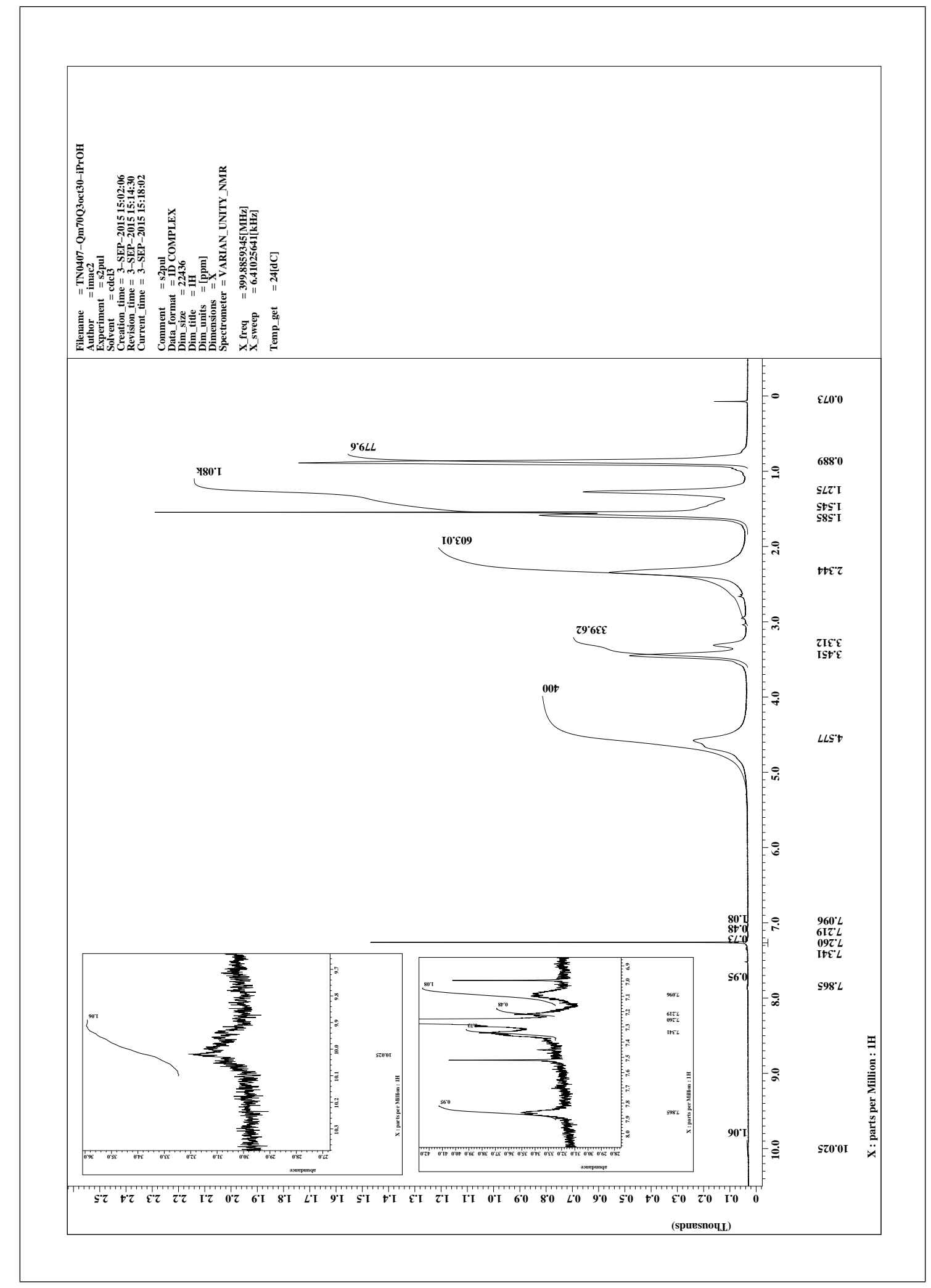

Figure S219. ${ }^{1} \mathrm{H}$ NMR spectrum of $\mathbf{3 d}(\mathbf{3 0} / 70)$ in $\mathrm{CDCl}_{3}$. 


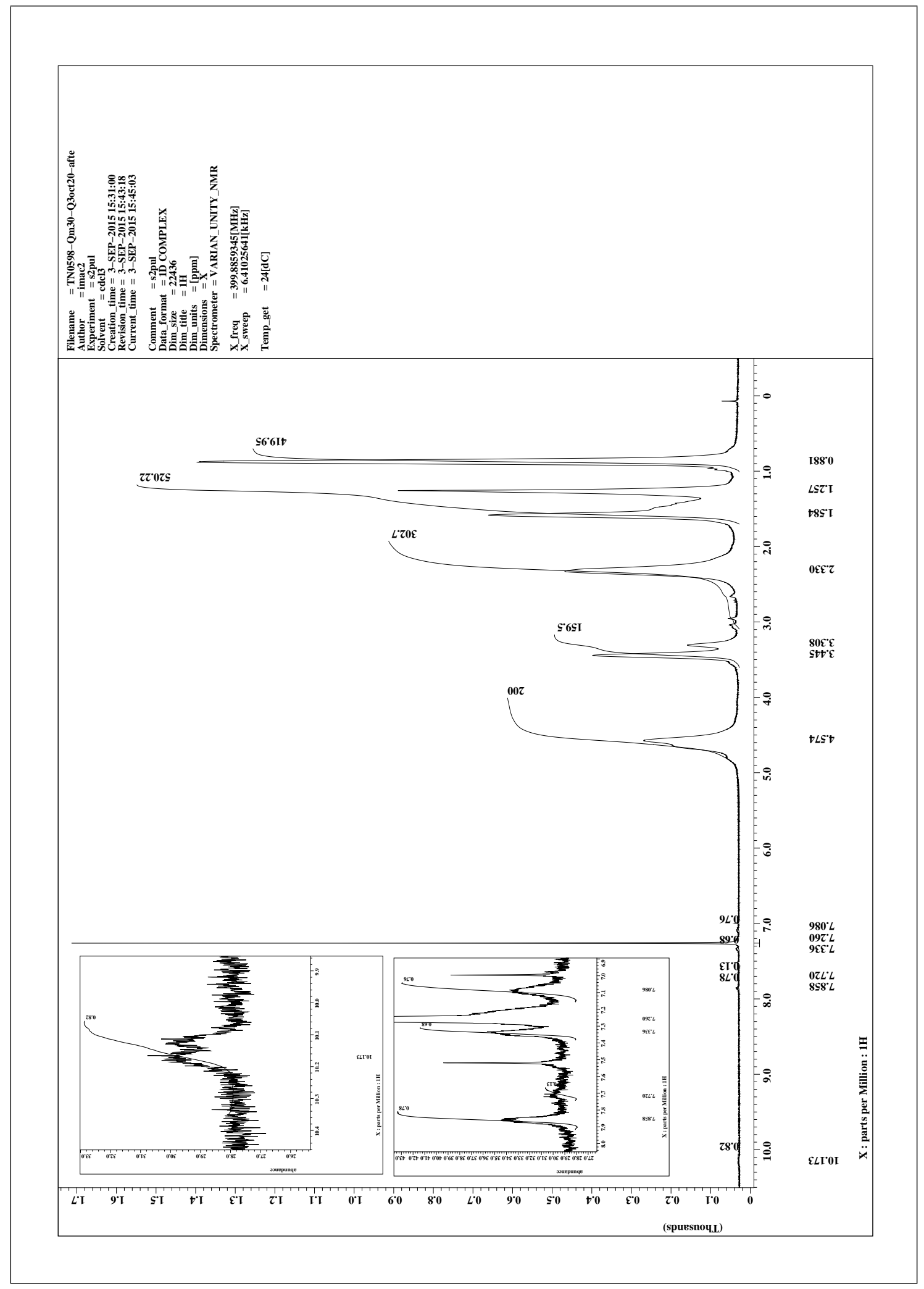

Figure S220. ${ }^{1} \mathrm{H}$ NMR spectrum of $\mathbf{3 d}(\mathbf{2 0 / 3 0})$ in $\mathrm{CDCl}_{3}$. 


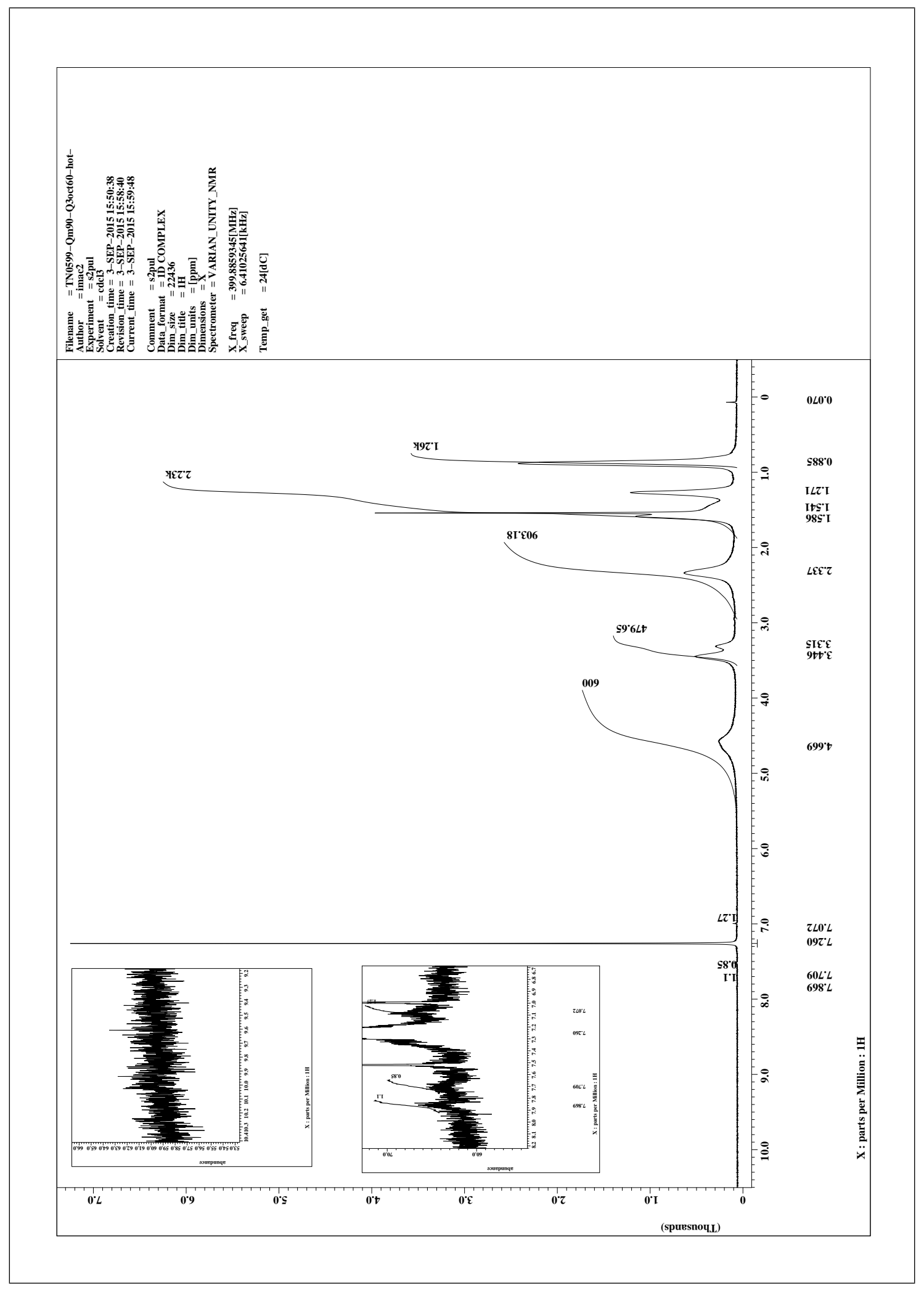

Figure S221. ${ }^{1} \mathrm{H}$ NMR spectrum of $\mathbf{3 d}(60 / 90)$ in $\mathrm{CDCl}_{3}$. 


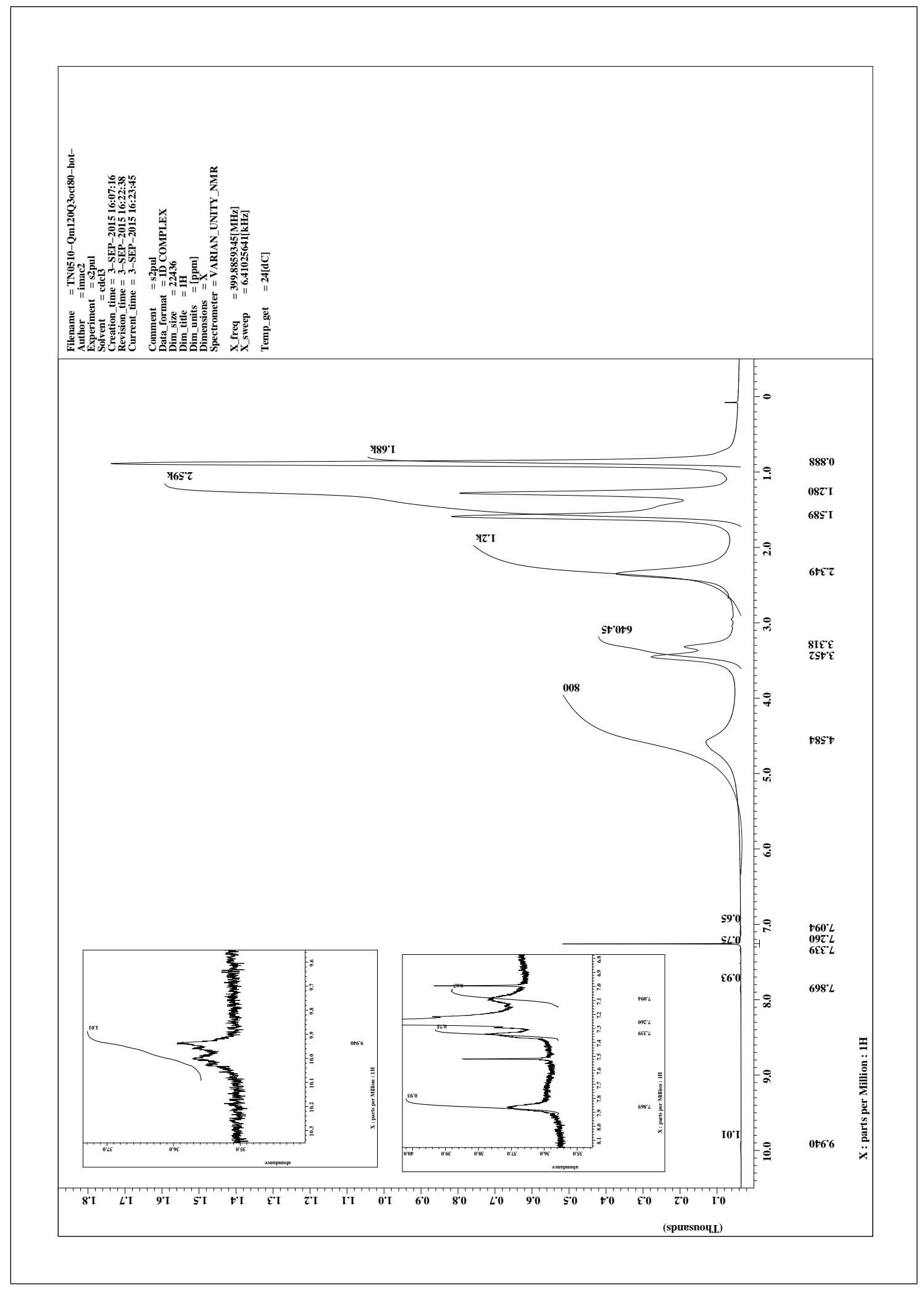

Figure S222. ${ }^{1} \mathrm{H}$ NMR spectrum of $\mathbf{3 d}(\mathbf{8 0} / \mathbf{1 2 0})$ in $\mathrm{CDCl}_{3}$. 


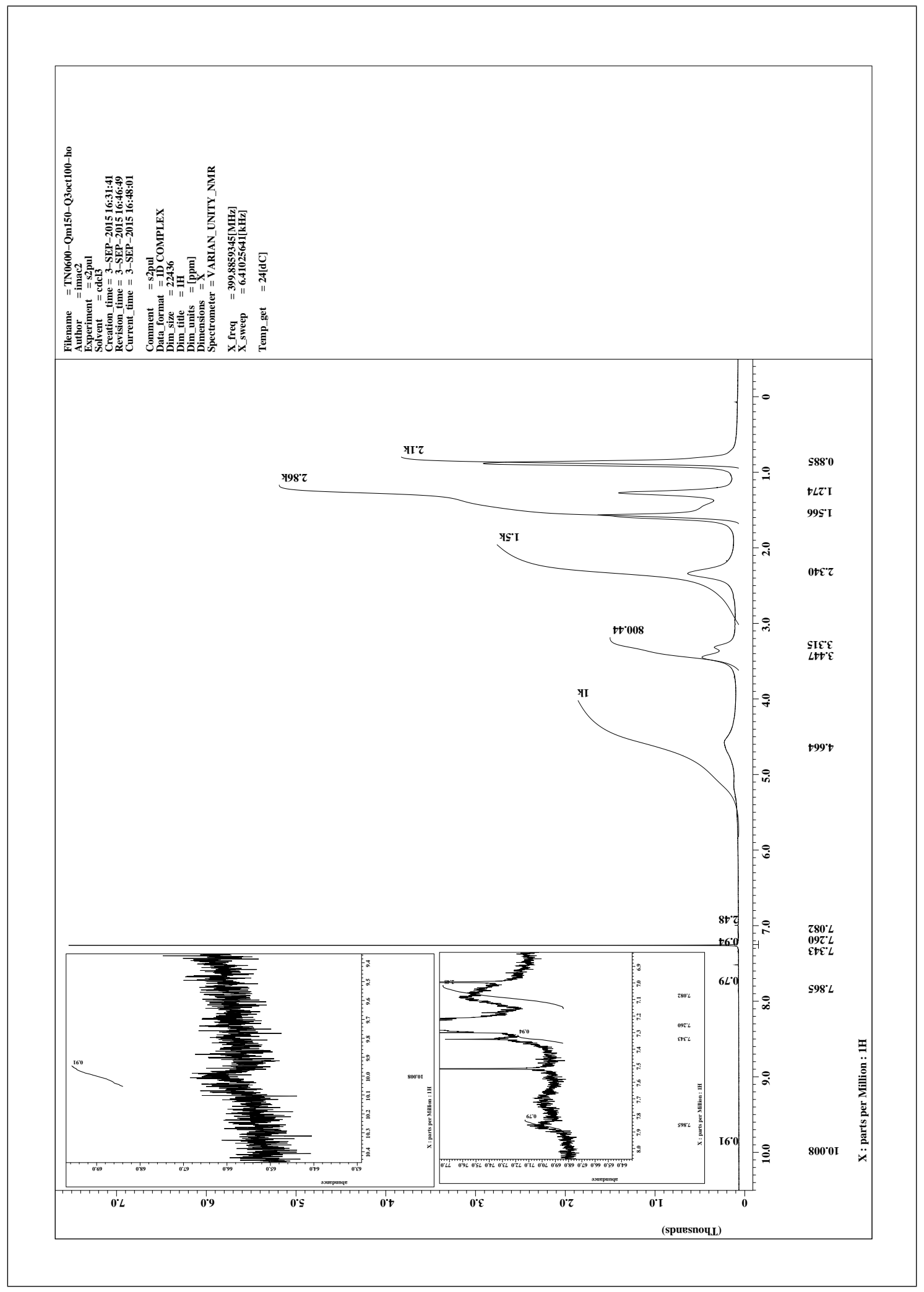

Figure S223. ${ }^{1} \mathrm{H}$ NMR spectrum of $\mathbf{3 d}(\mathbf{1 0 0 / 1 5 0})$ in $\mathrm{CDCl}_{3}$. 


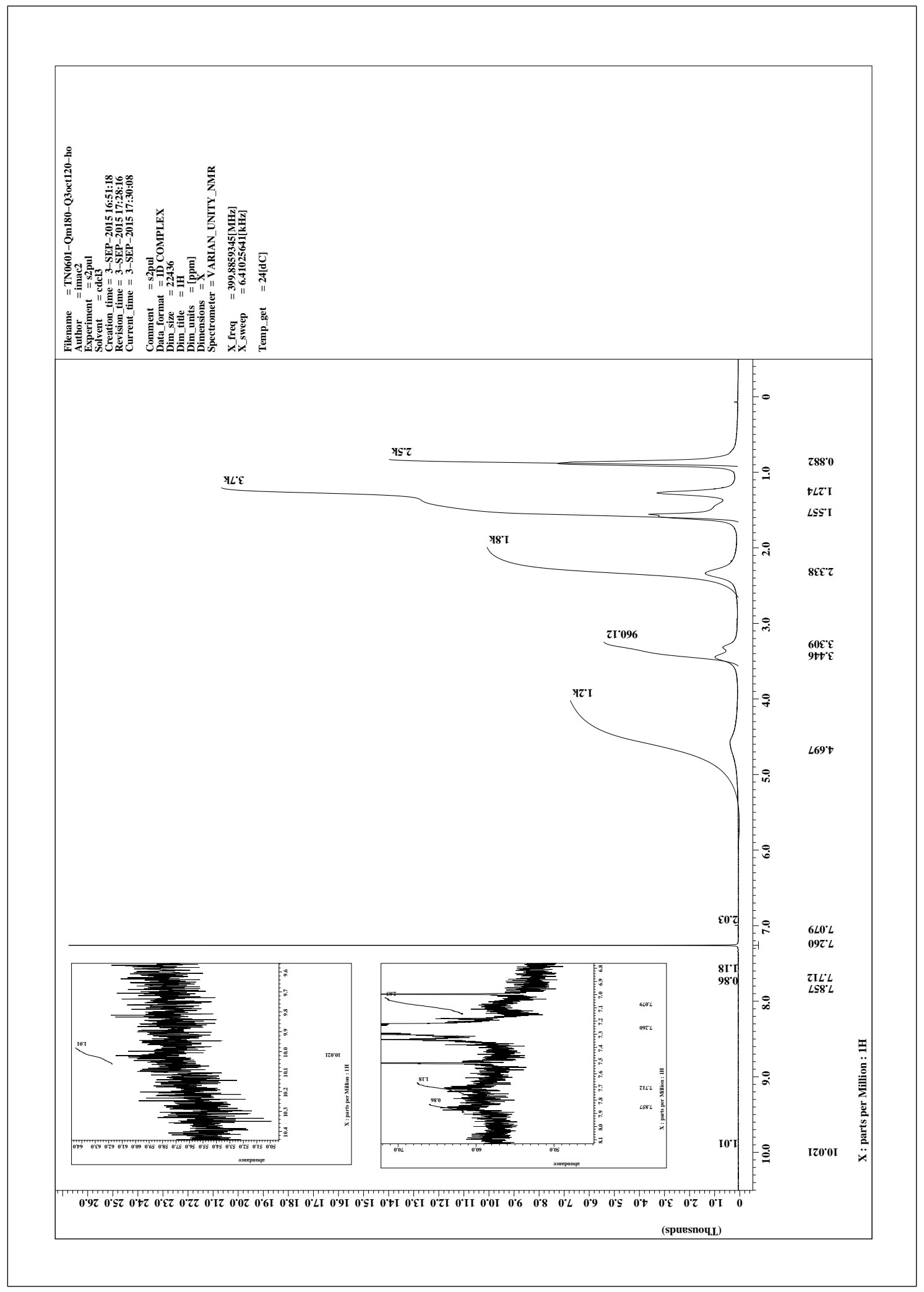

Figure S224. ${ }^{1} \mathrm{H}$ NMR spectrum of $\mathbf{3 d}(\mathbf{1 2 0 / 1 8 0})$ in $\mathrm{CDCl}_{3}$. 


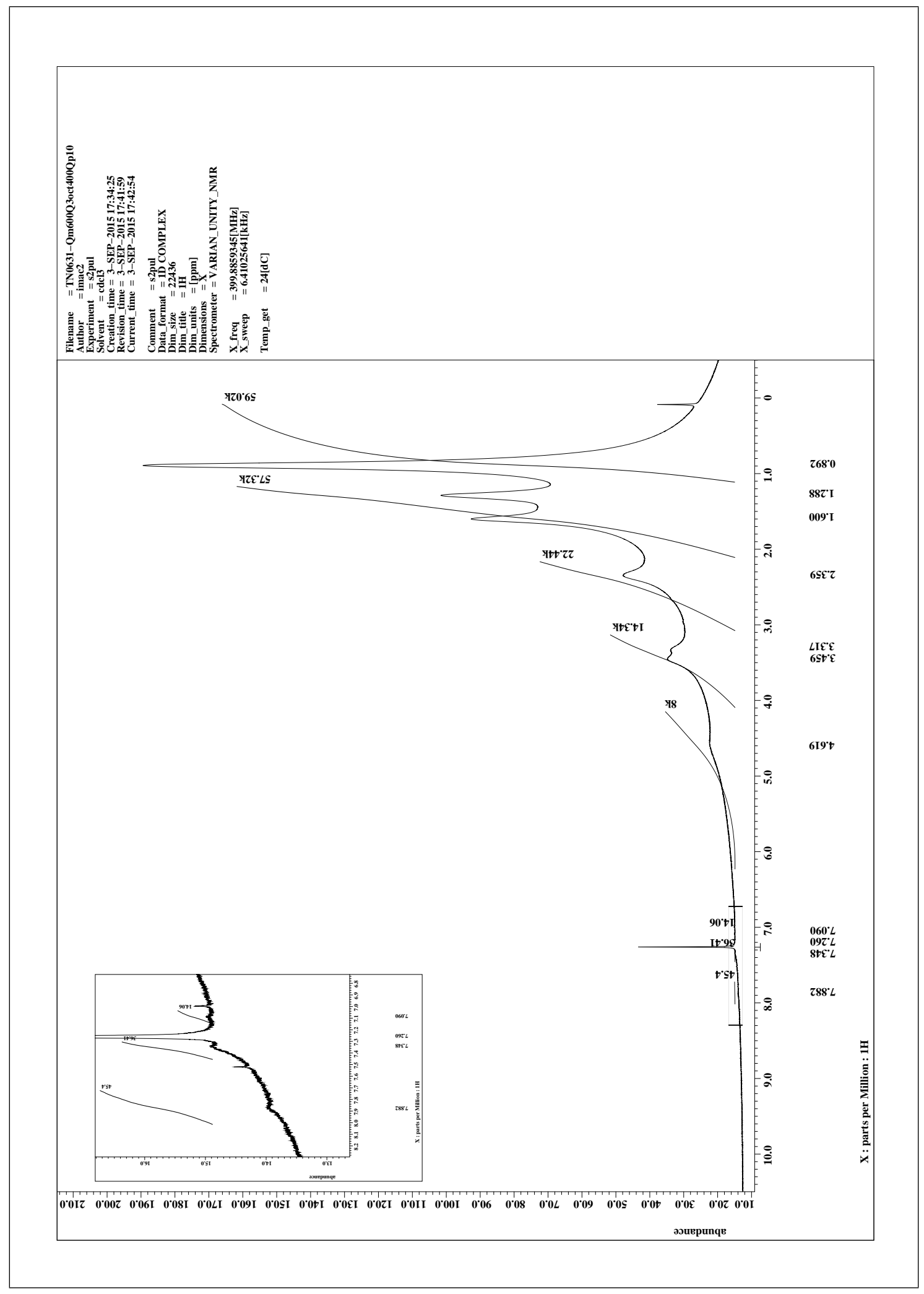

Figure S225. ${ }^{1} \mathrm{H}$ NMR spectrum of 4 in $\mathrm{CDCl}_{3}$. 


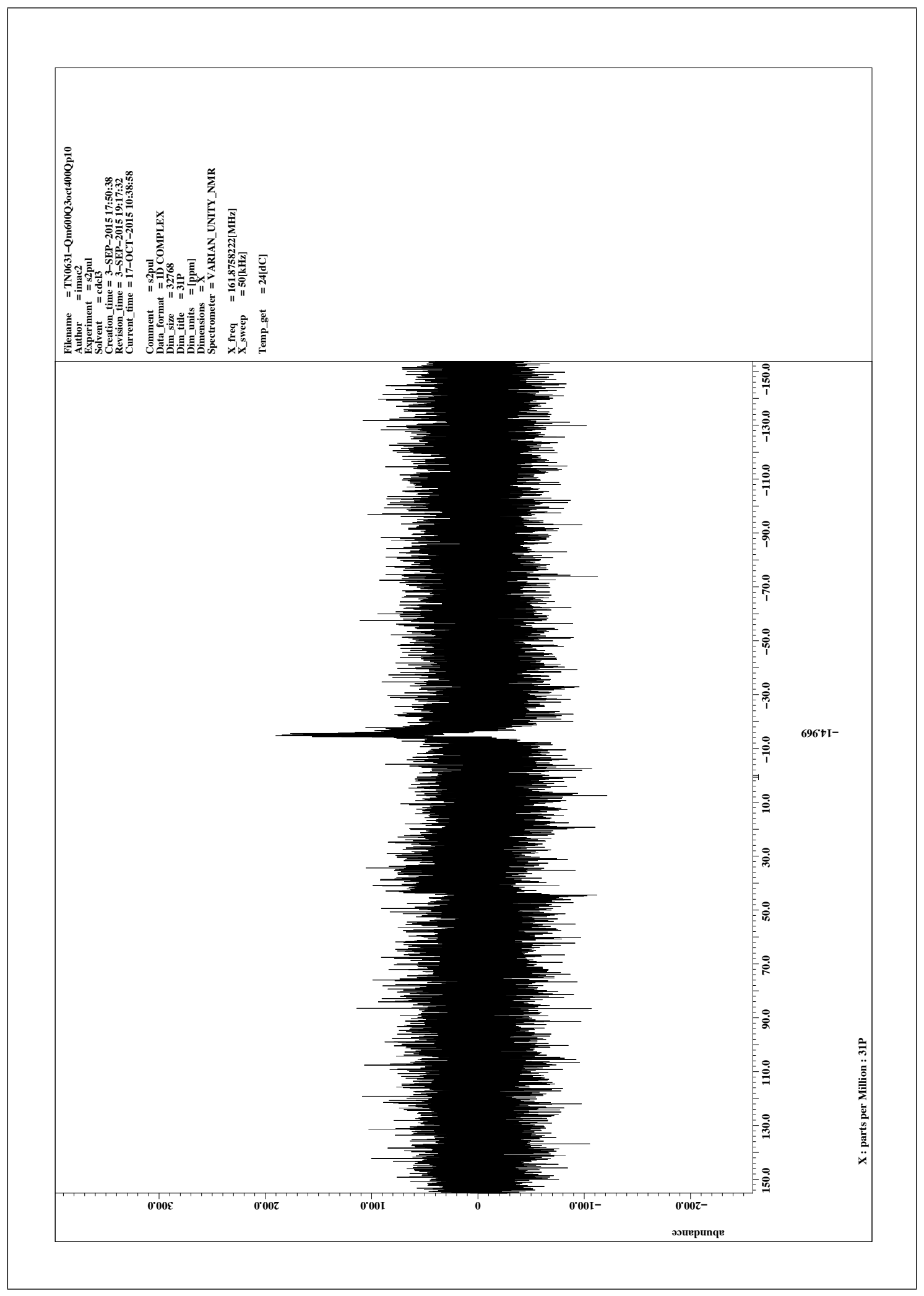

Figure S226. ${ }^{31} \mathrm{P}$ NMR spectrum of 4 in $\mathrm{CDCl}_{3}$. 


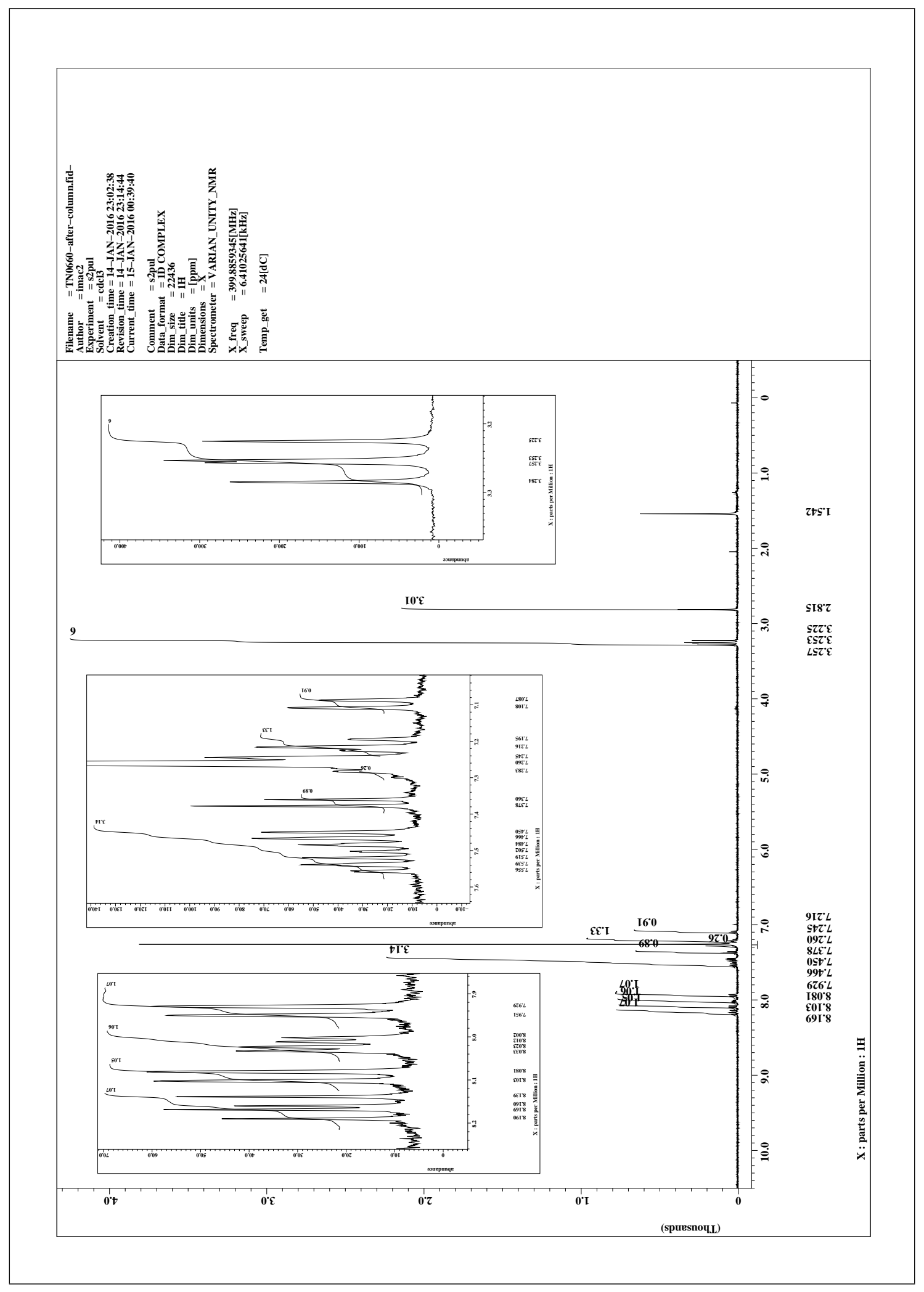

Figure S227. ${ }^{1} \mathrm{H}$ NMR spectrum of 4 in $\mathrm{CDCl}_{3}$. 


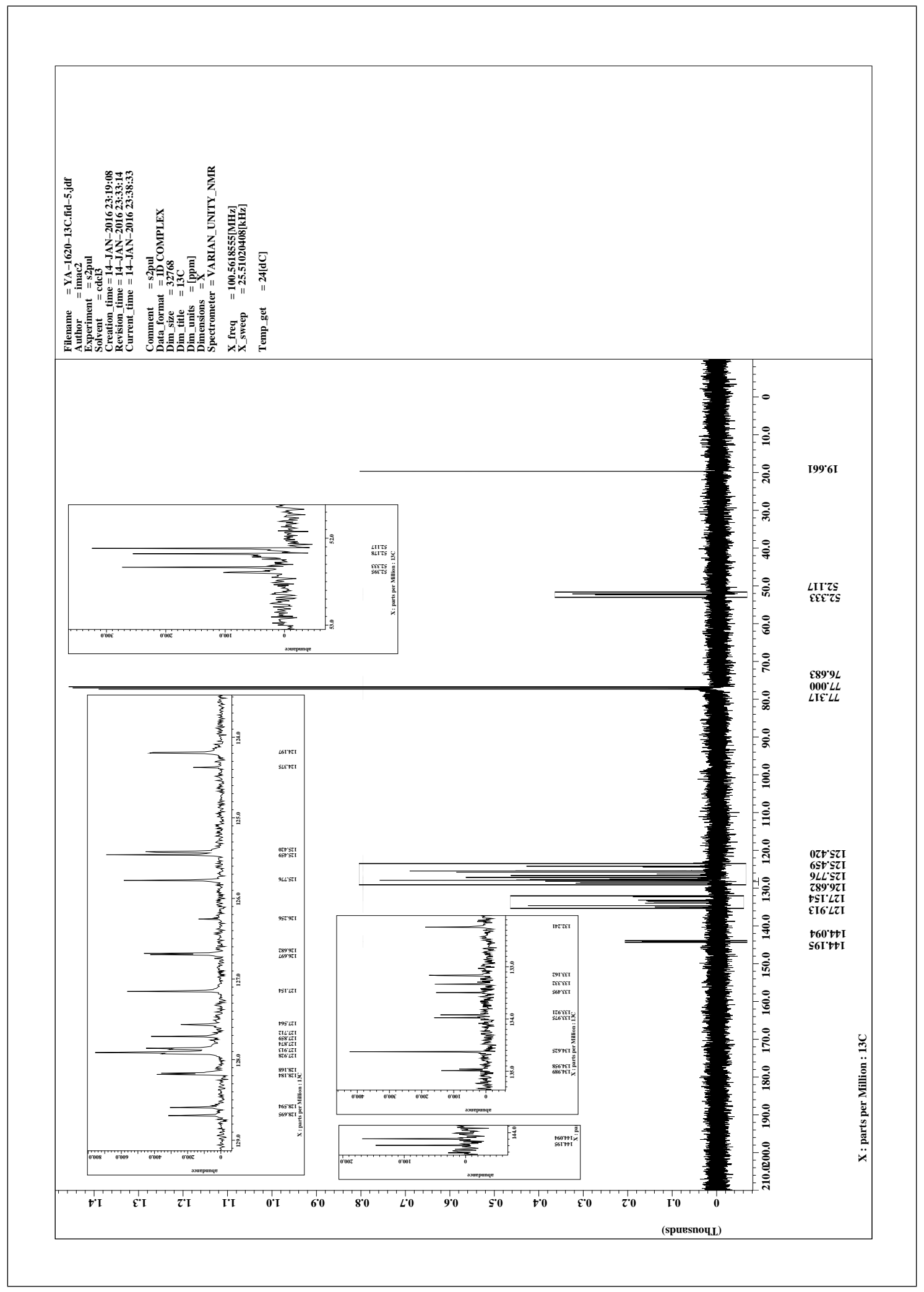

Figure S228. ${ }^{13} \mathrm{C}$ NMR spectrum of 4 in $\mathrm{CDCl}_{3}$. 


$$
\exists
$$

